\title{
Determination of the Chemical Compositions of Condensate- like Oils with Different API Gravities by Using the Distillation, Precipitation, Fractionation Mass Spectrometry (DPF MS) Method
}

Kawthar Z. Alzarieni, ${ }^{a}$ Yanfen Zhang, ${ }^{b}$ Edouard Niyonsaba, ${ }^{a}$ Katherine E. Wehde, ${ }^{a}$ Cliff T. Johnston, ${ }^{\mathrm{c}}$ Gozdem Kilaz, ${ }^{\text {d }}$ and Hilkka I. Kenttämaa ${ }^{\mathrm{a},{ }^{*}}$

${ }^{a}$ Department of Chemistry, Purdue University, West Lafayette, IN 47907, United States

' State Key Laboratory of Heavy Oil Processing, China University of Petroleum, Beijing 102249, PR China

${ }^{c}$ Department of Agronomy, Purdue University, West Lafayette, IN 47907, United States

${ }^{d}$ Department of Engineering Technology, Purdue University, West Lafayette, IN 479-7

*Corresponding author:

Hilkka I. Kenttämaa

Address: Department of Chemistry, Purdue University, West Lafayette, IN 47907, USA.

Tel.: +1 (765) 494 0882; fax: +1 (765) 4949421.

E-mail: hilkka@purdue.edu

Submitted to Energy and Fuels 


\section{$\underline{\text { List of contents }}$}

\section{$\underline{\text { Figures }}$}

Figure S1. Mass calibration spectra of LQIT/orbitrap MS and GC $\times$ GC/TOF MS .....

Figure S2. The average weight percent of compounds in the alkyl aromatic hydrocarbon class as a function of API gravity for five different condensate-like oils.

Figure S3. The average weight percent of compounds in the volatile hydrocarbon class as a function of API gravity for five different condensate-like oils.

Figure S4. The average weight percent of polar compounds as a function of API gravity for five different condensate-like oils. 10

Figure S5. The average weight percent of compounds with the elemental composition $\mathrm{C}_{c} \mathrm{H}_{\mathrm{h}}$ as a function of API gravity for five different condensate-like oils......

Figure S6. The average weight percent of compounds with the elemental composition $\mathrm{C}_{c} \mathrm{H}_{\mathrm{h}} \mathrm{N}_{\mathrm{n}}$ as a function of API gravity for five different condensate-like oils.

Figure S7. The average weight percent of compounds with the elemental composition $\mathrm{C}_{c} \mathrm{H}_{h} \mathrm{O}_{\mathrm{o}}$ as a function of API gravity for five different condensate-like oils.

Figure S8. The average weight percent of compounds with the elemental composition $\mathrm{C}_{\mathrm{c}} \mathrm{H}_{\mathrm{h}} \mathrm{O}_{\mathrm{o}} \mathrm{S}_{\mathrm{s}}$ as a function of API gravity for five different condensate-like oils.

Figure S9. The average weight percent of compounds with the elemental composition $\mathrm{C}_{c} \mathrm{H}_{h} \mathrm{~N}_{n} \mathrm{O}_{\mathrm{o}}$ as a function of API gravity for five different condensate-like oils.

Figure S10. The average weight percent of compounds with the elemental composition $\mathrm{C}_{\mathrm{c}} \mathrm{H}_{\mathrm{h}} \mathrm{N}_{\mathrm{n}} \mathrm{O}_{\mathrm{o}} \mathrm{S}_{\mathrm{s}}$ as a function of API gravity for five different condensate-like oils.

Figure S11. The average weight percent of compounds with the elemental composition type $\mathrm{C}_{c} \mathrm{H}_{h} \mathrm{~N}_{\mathrm{n}} \mathrm{O}_{\mathrm{o}} \mathrm{S}_{\mathrm{s}}$ in each heteroaromatic compound class as a function of API gravity for five different condensate-like oils.

Figure S12. The average weight percent of compounds with the elemental composition type $\mathrm{C}_{\mathrm{c}} \mathrm{H}_{\mathrm{h}} \mathrm{O}_{\mathrm{o}} \mathrm{S}_{\mathrm{s}}$ in each heteroaromatic compound class as a function of API gravity for five different condensate-like oils.

Figure S13. The average weight percent of compounds with the elemental composition type $\mathrm{C}_{c} \mathrm{H}_{h} \mathrm{~N}_{n} \mathrm{O}_{\mathrm{o}}$ in each alkyl aromatic compound class as a function of API gravity for five different condensate-like oils.

Figure S14. The average weight percent of compounds with the elemental composition type $\mathrm{C}_{c} \mathrm{H}_{\mathrm{h}} \mathrm{O}_{\mathrm{o}}$ in each heteroaromatic compound class as a function of API gravity for five different condensate-like oils.

Figure S15. The average weight percent of compounds with the elemental composition $\mathrm{C}_{c} \mathrm{H}_{\mathrm{h}} \mathrm{S}_{\mathrm{s}}$ in each alkyl aromatic hydrocarbon class as a function of API gravity of five different condensate-like oils.

Figure S16. The average weight percent of compounds with the elemental composition $\mathrm{C}_{c} \mathrm{H}_{\mathrm{h}} \mathrm{N}_{\mathrm{n}} \mathrm{O}_{\mathrm{o}} \mathrm{S}_{\mathrm{s}}$ in each alkyl aromatic compound class as a function of API gravity of five different condensate-like oils.

Figure S17. The average weight percent of compounds with the elemental composition $\mathrm{C}_{c} \mathrm{H}_{\mathrm{h}} \mathrm{N}_{\mathrm{n}} \mathrm{O}_{\mathrm{o}} \mathrm{S}_{\mathrm{s}}$ in each polar compound class as a function of API gravity of five different condensate-like oils.

Figure S18. The average weight percent of compounds with the elemental composition $\mathrm{C}_{c} \mathrm{H}_{\mathrm{h}} \mathrm{N}_{\mathrm{n}} \mathrm{O}_{\mathrm{o}} \mathrm{S}_{\mathrm{s}}$ in each heavy saturated hydrocarbon class as a function of API gravity of five different condensate-like oils... 
Figure S19. The average weight percent of compounds with the elemental composition $\mathrm{C}_{c} \mathrm{H}_{\mathrm{h}} \mathrm{N}_{\mathrm{n}} \mathrm{O}_{\mathrm{o}} \mathrm{S}_{\mathrm{s}}$ in each volatile hydrocarbon class as a function of API gravity of five different condensate-like oils.

Figure S20. The average weight percent of compounds with the elemental composition $\mathrm{C}_{c} \mathrm{H}_{\mathrm{h}} \mathrm{N}_{\mathrm{n}} \mathrm{O}_{\mathrm{o}}$ in each polar compound class as a function of API gravity of five different condensate-like oils.

Figure S21. The average weight percent of compounds with the elemental composition $\mathrm{C}_{c} \mathrm{H}_{\mathrm{h}} \mathrm{N}_{\mathrm{n}} \mathrm{O}_{\mathrm{o}}$ in each heteroaromatic compound class as a function of API gravity of five different condensate-like oils.

Figure S22. The average weight percent of compounds with the elemental composition $\mathrm{C}_{c} \mathrm{H}_{\mathrm{h}} \mathrm{N}_{\mathrm{n}} \mathrm{O}_{\mathrm{o}}$ in each saturated hydrocarbon class as a function of API gravity of five different condensate-like oils.

Figure S23. The average weight percent of compounds with the elemental composition $\mathrm{C}_{c} \mathrm{H}_{\mathrm{h}} \mathrm{N}_{\mathrm{n}} \mathrm{O}_{\mathrm{o}}$ in each volatile hydrocarbon class as a function of API gravity of five different condensate-like oils.

Figure S24. The average weight percent of compounds with the elemental composition $\mathrm{C}_{\mathrm{c}} \mathrm{H}_{\mathrm{h}} \mathrm{O}_{\mathrm{o}} \mathrm{S}_{\mathrm{s}}$ in each polar compound class as a function of API gravity of five different condensate-like oils.

Figure S25. The average weight percent of compounds with the elemental composition $\mathrm{C}_{c} \mathrm{H}_{\mathrm{h}} \mathrm{O}_{\mathrm{o}} \mathrm{S}_{\mathrm{s}}$ in each alkyl aromatic hydrocarbon class as a function of API gravity of five different condensate-like oils.

Figure S26. The average weight percent of compounds with the elemental composition $\mathrm{C}_{c} \mathrm{H}_{\mathrm{h}} \mathrm{O}_{\mathrm{o}} \mathrm{S}_{\mathrm{s}}$ in each heavy saturated hydrocarbon class as a function of API gravity of five different condensate-like oils.

Figure S27. The average weight percent of compounds with the elemental composition $\mathrm{C}_{\mathrm{c}} \mathrm{H}_{\mathrm{h}} \mathrm{O}_{\mathrm{o}} \mathrm{S}_{\mathrm{s}}$ in each volatile hydrocarbon class as a function of API gravity of five different condensate-like oils.

Figure S28. The average weight percent of compounds with the elemental composition $\mathrm{C}_{c} \mathrm{H}_{h} \mathrm{O}_{\mathrm{o}}$ in each polar compound class as a function of API gravity of five different condensate-like oils.

Figure S29. The average weight percent of compounds with the elemental composition $\mathrm{C}_{c} \mathrm{H}_{\mathrm{h}} \mathrm{O}_{\mathrm{o}}$ in each alkyl aromatic hydrocarbon class as a function of API gravity of five different condensate-like oils......

Figure S30. The average weight percent of compounds with the elemental composition $\mathrm{C}_{c} \mathrm{H}_{\mathrm{h}} \mathrm{O}_{\mathrm{o}}$ in each heavy saturated hydrocarbon class as a function of API gravity of five different condensate-like oils.

Figure S31. The average weight percent of compounds with the elemental composition $\mathrm{C}_{c} \mathrm{H}_{h} \mathrm{O}_{\mathrm{o}}$ in each volatile hydrocarbon class as a function of API gravity of five different condensate-like oils......

Figure S32. The average weight percent of compounds with the elemental composition $\mathrm{C}_{c} \mathrm{H}_{\mathrm{h}} \mathrm{N}_{\mathrm{n}}$ in each polar compound class as a function of API gravity of five different condensate-like oils.

Figure S33. The average weight percent of compounds with the elemental composition $\mathrm{C}_{c} \mathrm{H}_{h} \mathrm{~N}_{n}$ in each alkyl aromatic hydrocarbon class as a function of API gravity of five different condensate-like oils.

Figure S34. The average weight percent of compounds with the elemental composition $\mathrm{C}_{c} \mathrm{H}_{h} \mathrm{~N}_{n}$ in each heteroaromatic compound class as a function of API gravity of five different condensate-like oils.

Figure S35. The average weight percent of compounds with the elemental composition $\mathrm{C}_{c} \mathrm{H}_{h} \mathrm{~N}_{n}$ in each heavy saturated hydrocarbon class as a function of API gravity of five different condensate-like oils.

Figure S36. The average weight percent of compounds with the elemental composition $\mathrm{C}_{c} \mathrm{H}_{\mathrm{h}} \mathrm{N}_{\mathrm{n}}$ in each volatile hydrocarbon class as a function of API gravity of five different condensate-like oils.

Figure S37. The average weight percent of compounds with the elemental composition $\mathrm{C}_{c} \mathrm{H}_{\mathrm{h}} \mathrm{S}_{\mathrm{s}}$ in each polar compound class as a function of API gravity of five different condensate-like oils...... 
Figure S38. The average weight percent of compounds with the elemental composition $\mathrm{C}_{c} \mathrm{H}_{\mathrm{h}} \mathrm{S}_{\mathrm{s}}$ in each heteroaromatic compound class as a function of API gravity of five different condensate-like oils.

Figure S39. The average weight percent of compounds with the elemental composition $\mathrm{C}_{\mathrm{c}} \mathrm{H}_{\mathrm{h}} \mathrm{S}_{\mathrm{s}}$ in each heavy saturated hydrocarbon class as a function of API gravity of five different condensate-like oils.

Figure S40. The average weight percent of compounds with the elemental composition $\mathrm{C}_{c} \mathrm{H}_{\mathrm{h}} \mathrm{S}_{\mathrm{s}}$ in each volatile hydrocarbon class as a function of API gravity of five different condensate-like oils.

Figure S41. The average weight percent of compounds with the elemental composition $\mathrm{C}_{c} \mathrm{H}_{\mathrm{h}}$ in each polar compound class as a function of API gravity of five different condensate-like oils.

Figure S42. The average weight percent of compounds with the elemental composition $\mathrm{C}_{c} \mathrm{H}_{h}$ in each heavy saturated hydrocarbon class as a function of API gravity of five different condensate-like oils.

Figure S43. The average weight percent of compounds with the elemental composition $\mathrm{C}_{\mathrm{c}} \mathrm{H}_{\mathrm{h}}$ in each volatile hydrocarbon class as a function of API gravity of five different condensate-like oils.

Figure S44. The average weight percent of compounds with the elemental composition $\mathrm{C}_{c} \mathrm{H}_{\mathrm{h}}$ in heteroaromatic compound class as a function of API gravity of five different condensate-like oils.

Figure S45. The overall average molecular weight of the condensate-like oil samples as a function of API gravity....................35

Figure S46. GC $\times$ GC/TOF chromatogram for the heavy saturated hydrocarbon fraction of oil 5 .....

Figure S47. Color-mapped bubble plots of ring and double bond equivalent values (RDBE) versus carbon number for ionized

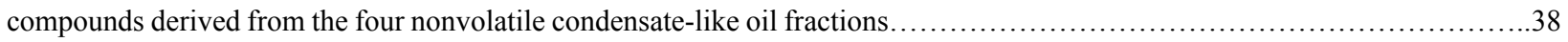

Figure S48. (+) APCI/ $\mathrm{N}_{2} / \mathrm{CS}_{2}$ mass spectra illustrating the ability to use ISCAD to cleave off most alkyl substituents in alkylaromatic hydrocarbons more than three alkyl chains..... ....39

Figure S49. (+) APCI/CS 2 mass spectra after ISCAD for the ionized compounds in the alkyl aromatic hydrocarbon classes of the five condensate-like oils. . .40

Figure S50. Ring and double bond equivalent (RDBE) versus carbon number plot for APCI mass spectrum (black) and ISCAD spectrum (red) of the alkyl aromatic hydrocarbons fraction of condensate-like oil 1.

\section{$\underline{\text { Tables }}$}

Table S1. List of model compounds and their percent mass purities.

Table S2. LQIT/orbitrap MS and GC $\times$ GC/TOF MS mass calibration data.

Table S3. Gravimetric weight percentages of the five different compound classes obtained using the DPF method for five condensate-like oil samples.

Table S4. The elemental composition types for the compounds in the different classes for five different condensate-like oils.....13

Table S5. The total wt $\%$ of nitrogen $(\mathrm{N})$, sulfur $(\mathrm{S})$ and oxygen $(\mathrm{O})$ containing compounds recovered from all fractions of the condensate-like oils.

Table S6. Elemental composition types of the compounds in the volatile saturated hydrocarbon class derived from five different condensate-like oils

Table S7. Elemental composition types of the compounds in the heavy saturated hydrocarbons class derived from five different condensate-like oils and the relative abundances of the ionized compounds based on mass spectrometry...... 
Table S8. Elemental composition types of the compounds in the alkyl aromatic hydrocarbons class derived from five different condensate-like oils and the relative abundances of the ionized compounds based on mass spectrometry.

Table S9. Elemental composition types of the compounds in the heteroaromatic compound class derived from five different condensate-like oils and the relative abundances of the ionized compounds based on mass spectrometry.

Table S10. Elemental composition types of the compounds in polar compounds class derived from five different condensate-like oils and the relative abundances of the ionized compounds based on mass spectrometry.

Table S11. The average molecular weights of compounds in the different compound classes and their standard deviations (SD; based on two measurements) for five different condensate-like oils.

Table S12. The RDBE values of compounds in the different compound classes of five different condensate-like oils

Table S13. The MW of the aromatic cores and the total number of carbons in the alkyl side chains of the alkyl aromatic hydrocarbons with averages and standard deviations from two experiments for the five condensate-like oils.

Table S14. Data obtained for compounds in the distillate fraction of condensate-like oil 2 by using GCxGC/(+EI) TOF MS......41

Table S15. Data obtained for the distillate fraction of condensate-like oil 1 by using GCxGC/(+EI) TOF MS...........................58

Table S16. Data obtained for the distillate fraction of condensate-like oil 5 by using GCxGC/(+EI)-TOF MS ..........................74

Table S17. Data obtained for the distillate fraction of condensate-like oil 4 by using GCxGC/(+EI) TOF MS..........................87

Table S18. Data obtained from the distillate fraction of condensate-like oil 3 by using GCxGC/(+EI)-TOF MS .96 
Table S1. List of model compounds and their purities.

\begin{tabular}{ll}
\hline \multicolumn{2}{c}{ Model compounds } \\
\hline Chemical & Percent mass purity \\
n-hexylamine & $99 \%$ \\
Diethyl disulfide & $99 \%$ \\
Ethyl benzoate & $\geq 99 \%$ \\
Butyl sulfone & $99 \%$ \\
3-ethylcarbazole & $97 \%$ \\
1,3-diphenylisobenzofuran & $97 \%$ \\
2-methylthiophene & $98 \%$ \\
Cholestane & $\geq 97 \%$ \\
Nonylcyclohexane & $95 \%$ \\
Decylcyclohexane & $95 \%$ \\
Heptadecylbenzene & $95 \%$ \\
p-xylene & $\geq 99 \%$ \\
1,3,5-isopropylbenzene & $95 \%$ \\
Hexaethylbenzene & $95 \%$ \\
1,3,5-triethylbenzene & $\geq 97 \%$ \\
Indane & $95 \%$ \\
\hline
\end{tabular}

Table S2. Mass calibration for A) LQIT/orbitrap MS by using Pierce LTQ ESI positive ion calibration solution as the calibration agent on regular basis. B) $\mathrm{GC} \times \mathrm{GC} / \mathrm{TOF}$ MS by using perfluorotributylamine (PFTBA) as the calibration agent on the day the analyses were performed.

\begin{tabular}{lcl}
\hline A) & LQIT/orbitrap MS Mass Calibration Table \\
\hline Expected $m / z$ & Observed $m / z$ & $\begin{array}{l}\text { Error in measured } m / z \\
(\mathrm{ppm})\end{array}$ \\
195.08762 & 195.08763 & 0.05126 \\
524.26496 & 524.26494 & -0.03815 \\
1121.99702 & 1121.99805 & 0.91801 \\
1221.99064 & 1221.99043 & -0.17185 \\
1321.98425 & 1321.98430 & 0.03782 \\
1421.97786 & 1421.97708 & -0.54853 \\
1521.97148 & 1521.97283 & 0.88701 \\
1621.96509 & 1621.96573 & 0.39458 \\
1721.95870 & 1721.95769 & -0.58654 \\
1821.95231 & 1821.95404 & 0.94953 \\
\hline
\end{tabular}

\begin{tabular}{lcl}
\hline & B) & $\mathrm{GC} \times \mathrm{GC} / \mathrm{TOF}$ MS Mass Calibration Table \\
\hline Expected $\mathrm{m} / \mathrm{z}$ & Observed $\mathrm{m} / \mathrm{z}$ & $\begin{array}{l}\text { Error in measured } \mathrm{m} / \mathrm{z} \\
(\mathrm{ppm})\end{array}$ \\
68.99 & 68.99 & -0.05 \\
118.99 & 118.99 & 0.25 \\
130.99 & 130.99 & -0.17
\end{tabular}




\begin{tabular}{lll}
218.99 & 218.99 & -0.36 \\
263.99 & 263.99 & 0.24 \\
413.98 & 413.98 & 0.36 \\
501.97 & 501.97 & 0.01 \\
\hline
\end{tabular}

\section{A}
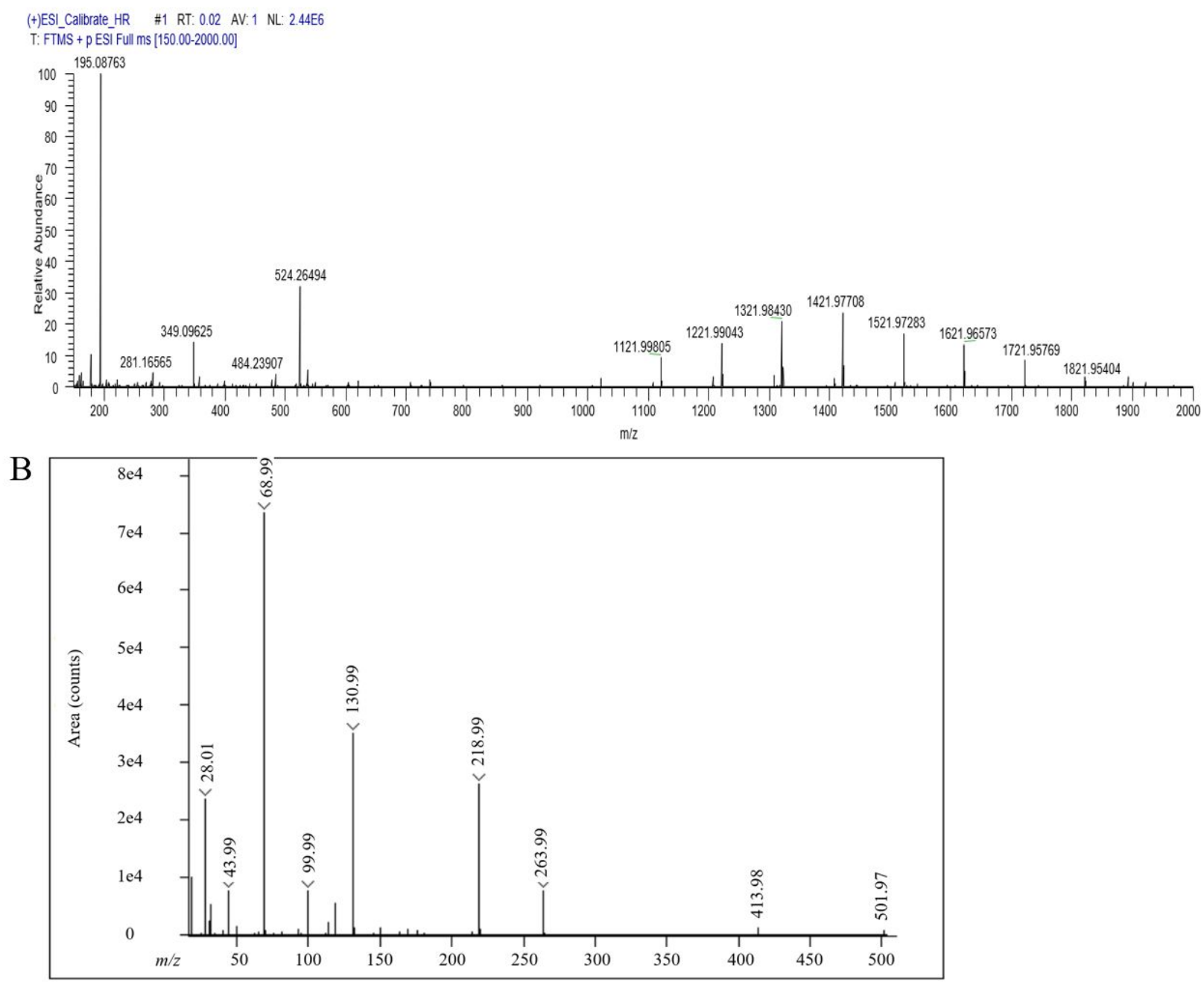

Figure S1. A) LQIT/orbitrap FTMS mass spectrum of the Pierce LTQ ESI positive ion calibration solution. B) GC $\times$ GC/TOF MS mass spectrum of the calibration agent perfluorotributylamine (PFTBA).

Table S3. Gravimetric weight percentages of the five different compound classes obtained using the DPF method for five condensate-like oil samples. Each experiment was performed twice. The averaged results, their standard deviations (SD) and $95 \%$ confidence intervals were determined based on these two trials. API gravities are shown in parenthesis.

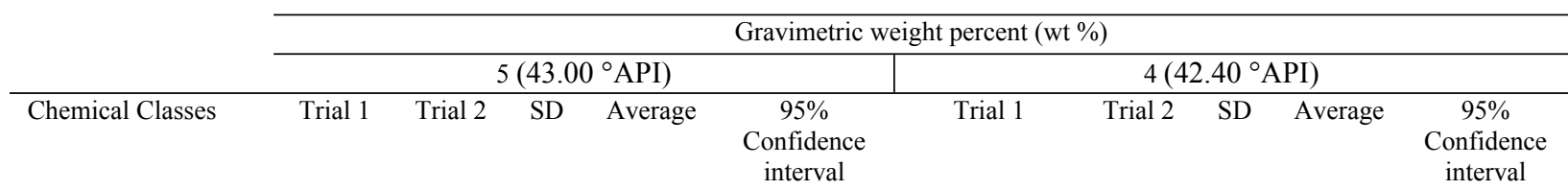




\begin{tabular}{|c|c|c|c|c|c|c|c|c|c|c|}
\hline $\begin{array}{l}\text { Volatile } \\
\text { Hydrocarbons }\end{array}$ & 0.76 & 0.76 & 0.00 & 0.76 & - & 1.31 & 1.31 & 0.00 & 1.31 & - \\
\hline $\begin{array}{l}\text { Heteroaromatic } \\
\text { Compounds }\end{array}$ & 0.41 & 0.49 & 0.06 & 0.45 & $0.37-0.53$ & 0.59 & 0.63 & 0.02 & 0.61 & $0.58-0.64$ \\
\hline Polar Compounds & 0.16 & 0.19 & 0.02 & 0.18 & $0.15-0.21$ & 1.39 & 0.75 & 0.32 & 1.06 & $0.62-1.5$ \\
\hline $\begin{array}{l}\text { Saturated } \\
\text { Hydrocarbons }\end{array}$ & 81.08 & 81.00 & 0.06 & 81.04 & $80.96-81.12$ & 78.44 & 77.69 & 0.38 & 78.06 & $77.53-78.59$ \\
\hline $\begin{array}{l}\text { Alkyl Aromatic } \\
\text { Hydrocarbons }\end{array}$ & 7.03 & 6.68 & 0.25 & 6.86 & $6.51-7.21$ & 6.05 & 7.17 & 0.56 & 6.61 & $5.83-7.39$ \\
\hline Loss & 10.55 & 10.87 & 0.23 & 10.71 & $10.39-11.03$ & 12.22 & 12.44 & 0.11 & 12.33 & $12.18-12.48$ \\
\hline Total & 100.00 & 100.00 & 0.00 & 100.00 & - & 100.00 & 100.00 & 0.00 & 100.00 & - \\
\hline
\end{tabular}

Table S3 continued

\begin{tabular}{|c|c|c|c|c|c|c|c|c|c|c|}
\hline \multirow[b]{3}{*}{ Chemical Classes } & \multicolumn{10}{|c|}{ Gravimetric weight percent (wt \%) } \\
\hline & \multicolumn{5}{|c|}{$3\left(41.62^{\circ} \mathrm{API}\right)$} & \multicolumn{5}{|c|}{$2\left(41.61^{\circ} \mathrm{API}\right)$} \\
\hline & Trial 1 & Trial 2 & SD & Average & $\begin{array}{c}95 \% \\
\text { Confidence } \\
\text { interval }\end{array}$ & Trial 1 & Trial 2 & SD & Average & $\begin{array}{c}95 \% \\
\text { Confidence } \\
\text { interval }\end{array}$ \\
\hline Volatile Hydrocarbons & 1.64 & 1.64 & 0.00 & 1.64 & - & 1.45 & 1.45 & 0.00 & 1.45 & - \\
\hline $\begin{array}{l}\text { Heteroaromatic } \\
\text { Compounds }\end{array}$ & 1.02 & 1.00 & 0.01 & 1.01 & $1.00-1.02$ & 0.91 & 1.09 & 0.10 & 1.00 & $0.86-1.14$ \\
\hline Polar Compounds & 0.28 & 0.22 & 0.04 & 0.25 & $0.19-0.31$ & 0.81 & 0.91 & 0.05 & 0.86 & $0.79-0.93$ \\
\hline $\begin{array}{l}\text { Saturated } \\
\text { Hydrocarbons }\end{array}$ & 76.10 & 75.74 & 0.25 & 75.92 & $75.57-76.27$ & 76.55 & 76.16 & 0.20 & 76.36 & $76.08-76.64$ \\
\hline $\begin{array}{l}\text { Alkyl Aromatic } \\
\text { Hydrocarbons }\end{array}$ & 13.21 & 10.93 & 1.61 & 12.07 & $9.84-14.30$ & 8.10 & 8.64 & 0.27 & 8.37 & $8.00-8.74$ \\
\hline Loss & 7.76 & 10.47 & 1.92 & 9.11 & $6.45-11.77$ & 12.19 & 11.75 & 0.22 & 11.97 & $11.67-12.27$ \\
\hline Total & 100.00 & 100.00 & 0.00 & 100.00 & - & 100.00 & 100.00 & 0.00 & 100.00 & - \\
\hline
\end{tabular}

Table $\mathbf{S 3}$ continued

\begin{tabular}{lccccc} 
& \multicolumn{4}{c}{ Gravimetric weight percent (wt \%) } \\
\cline { 2 - 5 } & Trial 1 & Trial 2 & SD & Average & $\begin{array}{c}95 \% \\
\text { Confidence } \\
\text { interval }\end{array}$ \\
\cline { 2 - 5 } Chemical Classes & & & & & - \\
Volatile Hydrocarbons & 2.07 & 2.07 & 0.00 & 2.07 & $3.04-3.34$ \\
Heteroaromatic Compounds & 3.27 & 3.11 & 0.11 & 3.19 & $1.41-1.77$ \\
Polar Compounds & 1.68 & 1.49 & 0.13 & 1.59 & $65.86-73.84$ \\
Saturated Hydrocarbons & 67.81 & 71.89 & 2.88 & 69.85 & $9.36-11.28$ \\
Alkyl Aromatic Hydrocarbons & 10.81 & 9.83 & 0.69 & 10.32 & $10.29-15.67$ \\
Loss & 14.35 & 11.61 & 1.94 & 12.98 & - \\
Total & 100.00 & 100.00 & 0.00 & 100.00 & \\
\hline
\end{tabular}




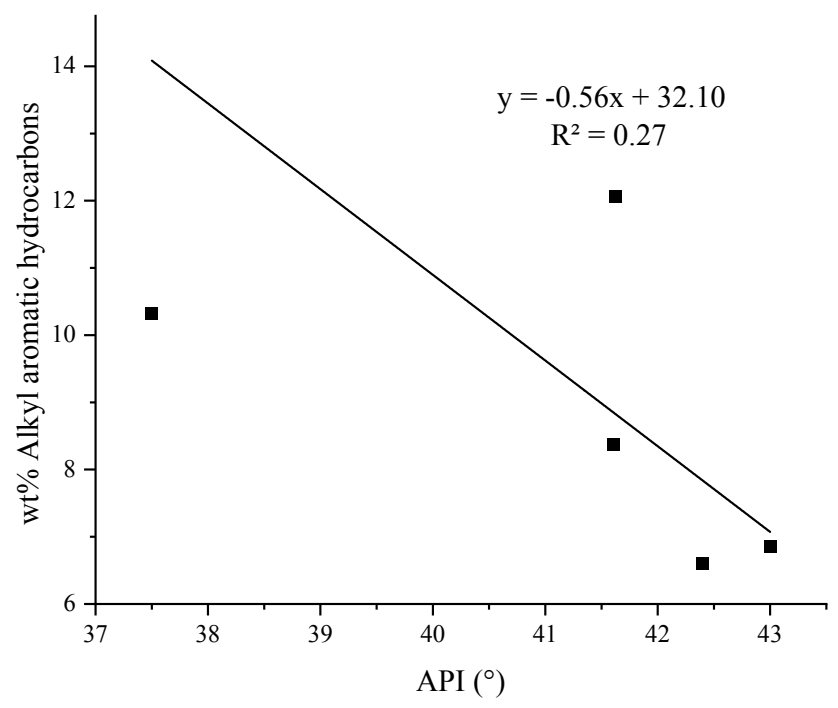

Figure S2. The average weight percent of compounds in the alkyl aromatic hydrocarbon class as a function of API gravity for five different condensate-like oils.

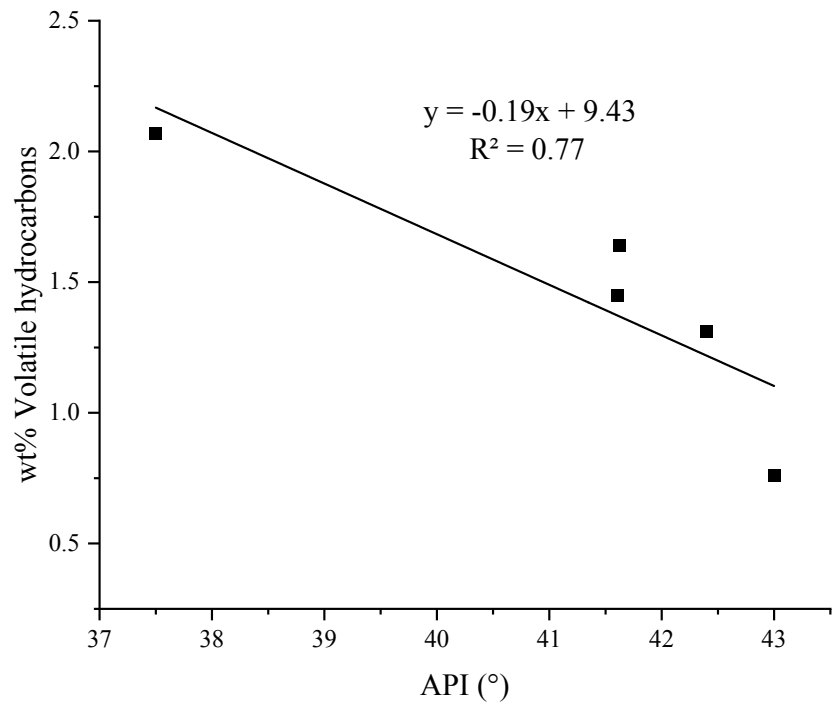

Figure S3. The average weight percent of compounds in the volatile hydrocarbon class as a function of API gravity for five different condensate-like oils. 


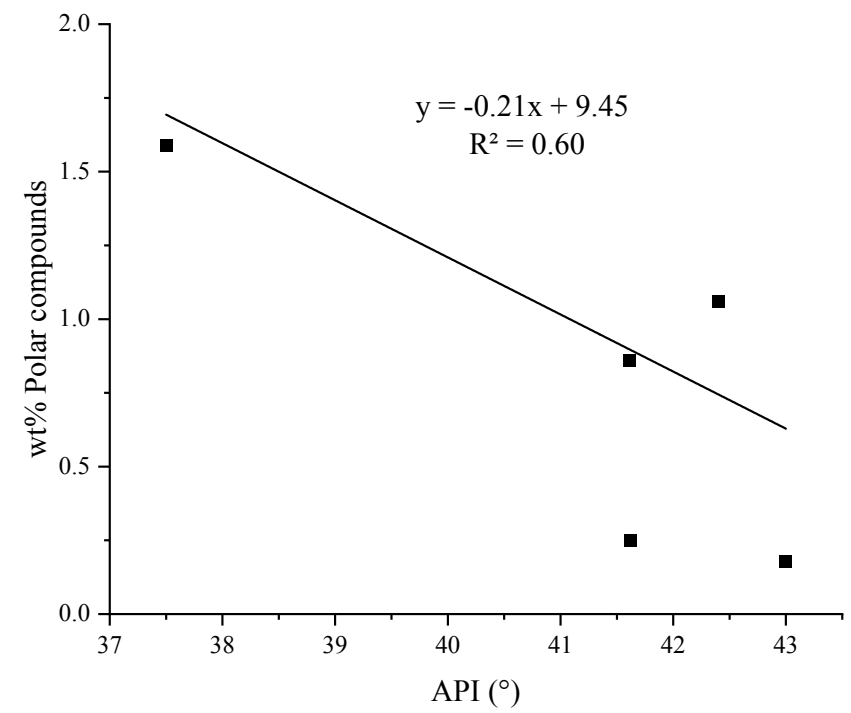

Figure S4. The average weight percent of polar compounds as a function of API gravity for five different condensate-like oils.

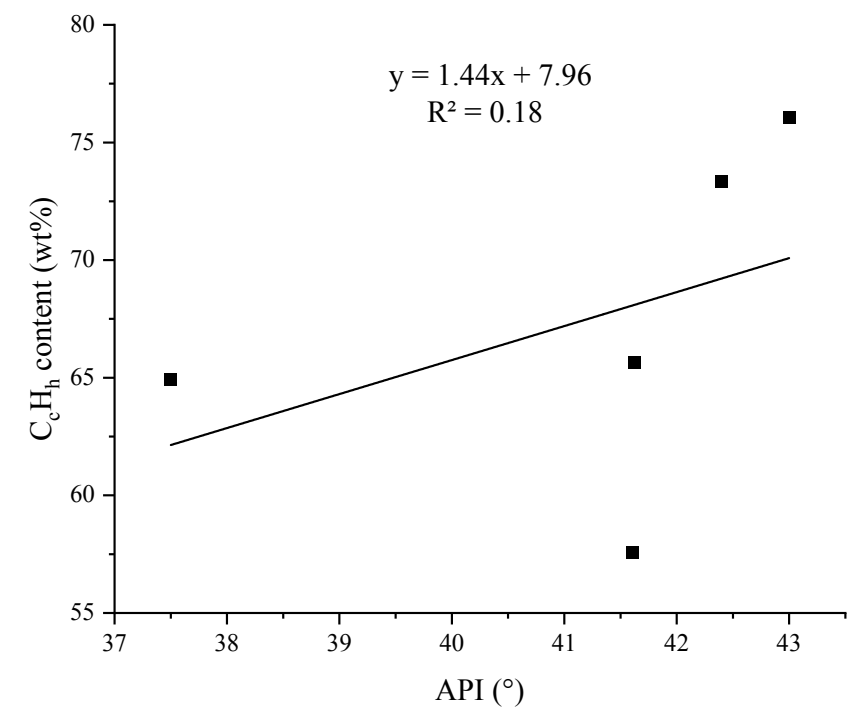

Figure S5. The average weight percent of compounds with the elemental composition $\mathrm{C}_{c} \mathrm{H}_{\mathrm{h}}$ as a function of API gravity for five different condensate-like oils. 


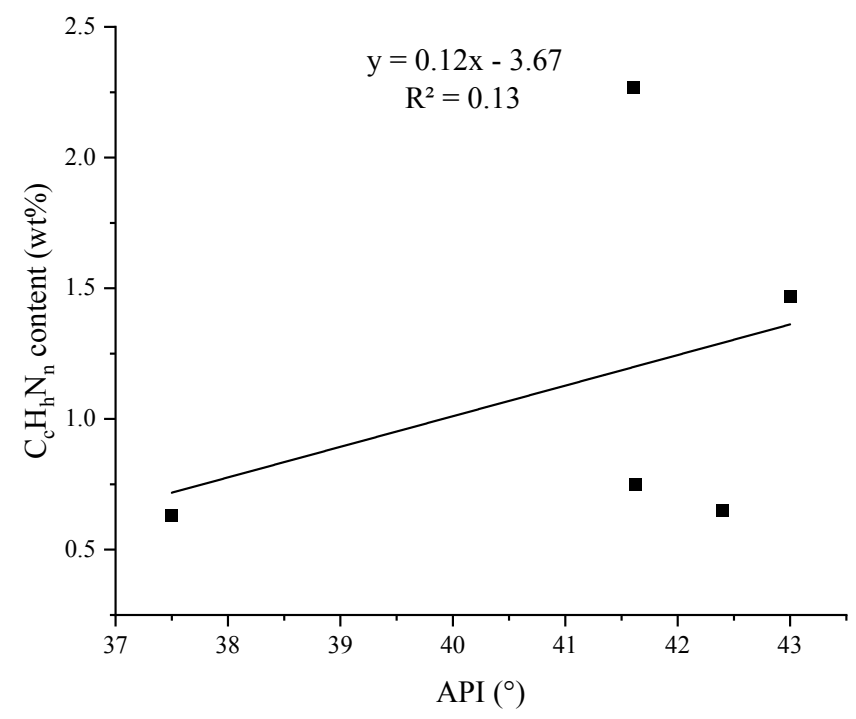

Figure S6. The average weight percent of compounds with the elemental composition $\mathrm{C}_{c} \mathrm{H}_{h} \mathrm{~N}_{n}$ as a function of API gravity for five different condensate-like oils.

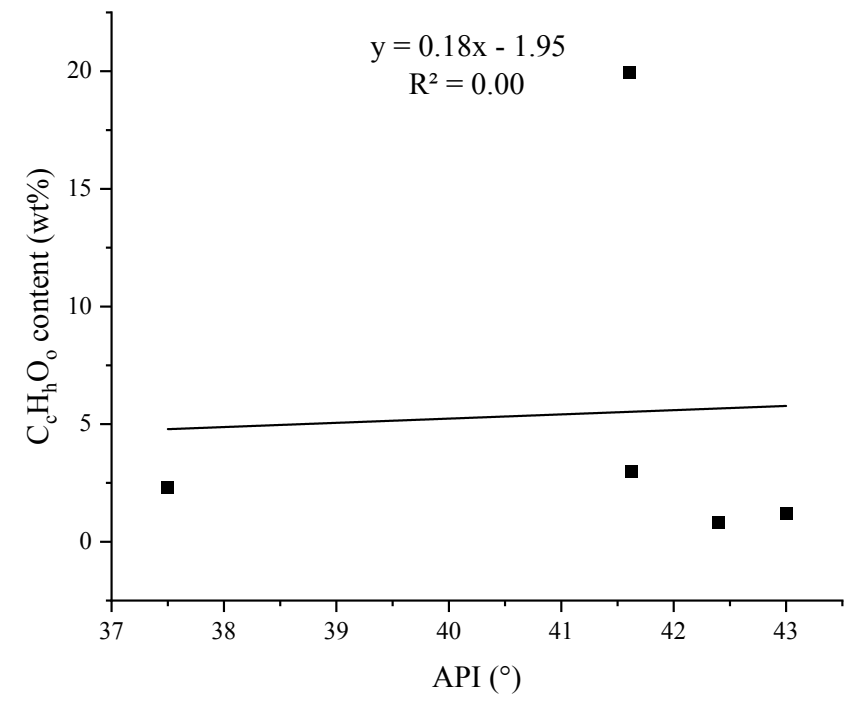

Figure S7. The average weight percent of compounds with the elemental composition $\mathrm{C}_{c} \mathrm{H}_{\mathrm{h}} \mathrm{O}_{\mathrm{o}}$ as a function of API gravity for five different condensate-like oils. 


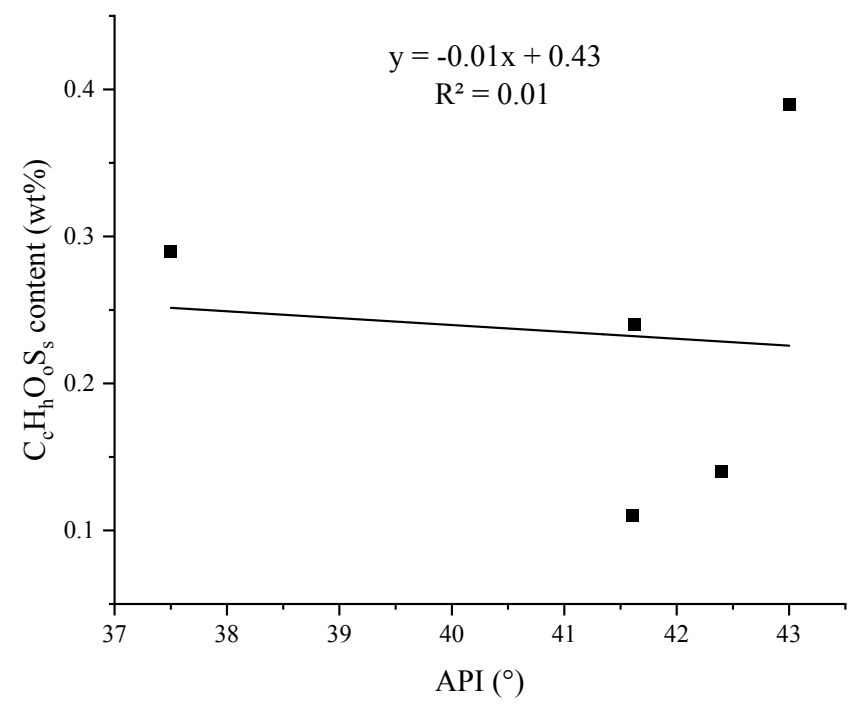

Figure S8. The average weight percent of compounds with the elemental composition $\mathrm{C}_{\mathrm{c}} \mathrm{H}_{\mathrm{h}} \mathrm{O}_{\mathrm{o}} \mathrm{S}_{\mathrm{s}}$ as a function of API gravity for five different condensate-like oils.

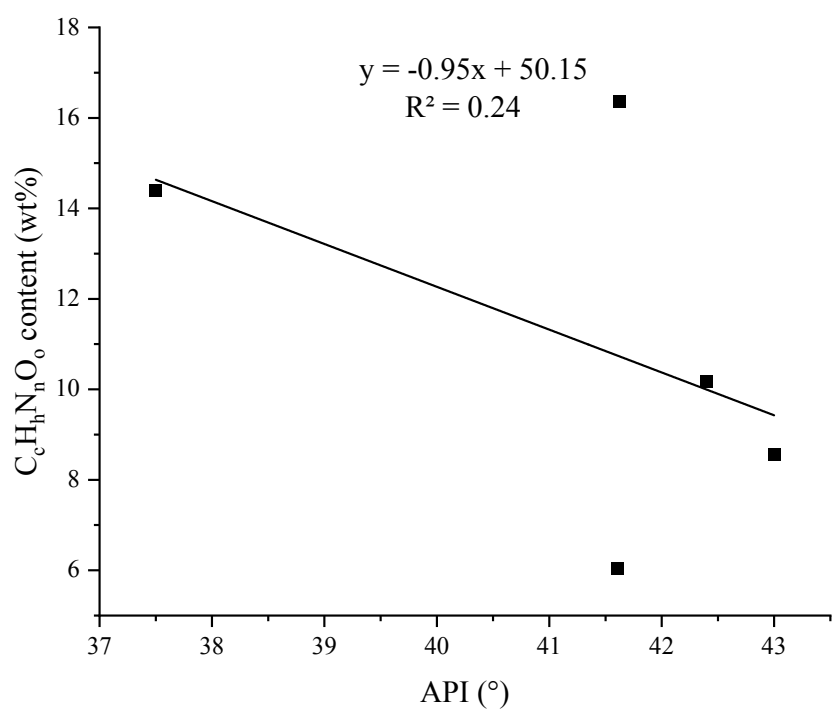

Figure S9. The average weight percent of compounds with the elemental composition $\mathrm{C}_{c} \mathrm{H}_{h} \mathrm{~N}_{n} \mathrm{O}_{\mathrm{o}}$ as a function of API gravity for five different condensate-like oils. 


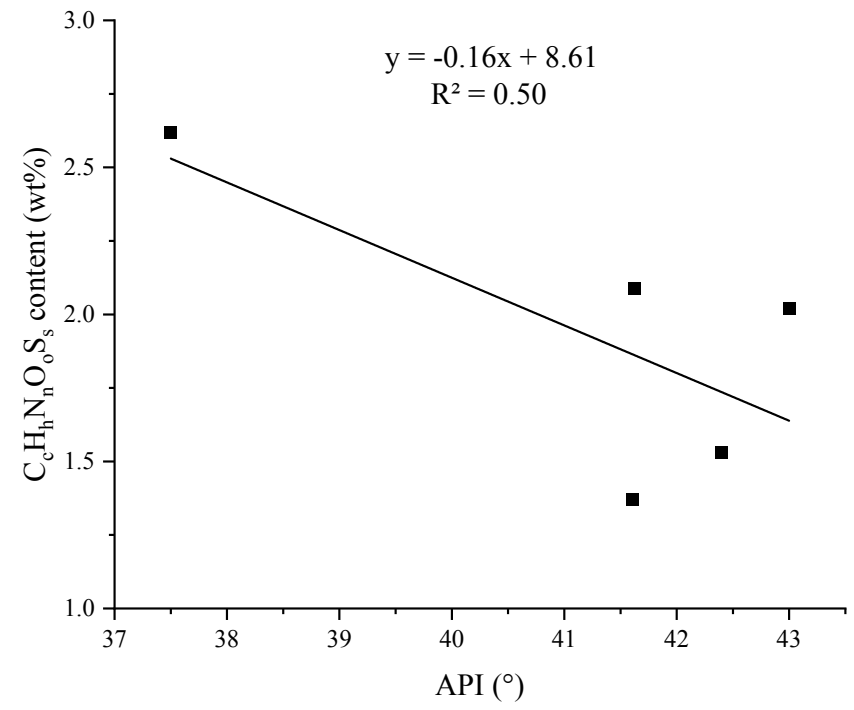

Figure S10. The average weight percent of compounds with the elemental composition $\mathrm{C}_{\mathrm{c}} \mathrm{H}_{\mathrm{h}} \mathrm{N}_{\mathrm{n}} \mathrm{O}_{\mathrm{o}} \mathrm{S}_{\mathrm{s}}$ as a function of API gravity for five different condensate-like oils.

Table S4. The elemental composition types for the compounds in the different classes for five different condensate-like oils. All data reported are averages of two trials. The total content of compounds of a specific chemical composition type in the condensatelike oil is the sum of the values measured in the five compound classes.

\begin{tabular}{|c|c|c|c|c|c|c|c|c|c|}
\hline \multirow[b]{2}{*}{$\begin{array}{l}\text { Condensate-like } \\
\text { Oil Sample }\end{array}$} & \multicolumn{7}{|c|}{ Semi-quantitative average wt $\%$} & \multirow[b]{2}{*}{$\begin{array}{c}\text { Standard } \\
\text { Deviation of } \\
\text { the Total } \\
\text { Content of } \\
\text { Compounds } \\
\text { with a } \\
\text { Specific } \\
\text { Elemental } \\
\text { Composition } \\
\text { Type }\end{array}$} & \multirow[b]{2}{*}{$\begin{array}{c}95 \% \\
\text { Confidence } \\
\text { interval }\end{array}$} \\
\hline & $\begin{array}{l}\text { Elemental } \\
\text { Composition } \\
\text { Type }\end{array}$ & $\begin{array}{c}\text { Volatile } \\
\text { Hydrocarbons }\end{array}$ & $\begin{array}{c}\text { Heavy } \\
\text { Saturated } \\
\text { Hydrocarbons }\end{array}$ & $\begin{array}{l}\text { Heteroaromatic } \\
\text { Compounds }\end{array}$ & $\begin{array}{c}\text { Alkyl } \\
\text { Aromatic } \\
\text { Hydrocarbons }\end{array}$ & $\begin{array}{c}\text { Polar } \\
\text { Compounds }\end{array}$ & $\begin{array}{c}\text { Total in the } \\
\text { Condensate- } \\
\text { like Oil }\end{array}$ & & \\
\hline 1 & \multirow{5}{*}{$\mathrm{C}_{\mathrm{c}} \mathrm{H}_{\mathrm{h}}$} & 1.40 & 53.10 & 0.00 & 10.30 & 0.10 & 65.00 & 1.30 & $63.2-66.8$ \\
\hline 2 & & 1.20 & 52.10 & 0.60 & 3.50 & 0.10 & 57.60 & 0.10 & $57.46-57.74$ \\
\hline 3 & & 1.20 & 58.80 & 0.70 & 4.80 & 0.10 & 65.70 & 0.00 & - \\
\hline 4 & & 1.00 & 69.20 & 0.50 & 2.60 & 0.10 & 73.40 & 0.80 & $66.21-80.59$ \\
\hline 5 & & 0.60 & 73.70 & 0.40 & 1.30 & 0.10 & 76.10 & 0.40 & $75.55-76.65$ \\
\hline 1 & \multirow{5}{*}{$\mathrm{C}_{\mathrm{c}} \mathrm{H}_{\mathrm{h}} \mathrm{S}_{\mathrm{s}}$} & 0.02 & 0.03 & 0.00 & 0.80 & 0.00 & 0.91 & 0.30 & $0.49-1.33$ \\
\hline 2 & & 0.06 & 0.00 & 0.01 & 0.20 & 0.00 & 0.30 & 0.10 & $0.16-0.44$ \\
\hline 3 & & 0.03 & 0.03 & 0.02 & 0.30 & 0.00 & 0.40 & 0.20 & $0.12-0.68$ \\
\hline 4 & & 0.09 & 0.00 & 0.00 & 0.20 & 0.00 & 0.30 & 0.10 & $0.16-0.44$ \\
\hline 5 & & 0.01 & 0.00 & 0.01 & 0.15 & 0.00 & 0.20 & 0.10 & $0.06-0.34$ \\
\hline 1 & \multirow{3}{*}{$\mathrm{C}_{\mathrm{c}} \mathrm{H}_{\mathrm{h}} \mathrm{N}_{\mathrm{n}}$} & 0.03 & 0.49 & 0.02 & 0.00 & 0.10 & 0.60 & 0.10 & $0.46-0.74$ \\
\hline 2 & & 0.00 & 1.87 & 0.00 & 0.20 & 0.20 & 2.30 & 0.00 & - \\
\hline 3 & & 0.02 & 0.25 & 0.01 & 0.20 & 0.30 & 0.80 & 0.10 & $0.66-0.94$ \\
\hline
\end{tabular}




\begin{tabular}{|c|c|c|c|c|c|c|c|c|c|}
\hline 4 & & 0.02 & 0.40 & 0.00 & 0.20 & 0.10 & 0.70 & 0.20 & $0.42-0.98$ \\
\hline 5 & & 0.02 & 1.09 & 0.00 & 0.30 & 0.10 & 1.50 & 0.40 & $0.95-2.05$ \\
\hline 1 & \multirow{5}{*}{$\mathrm{C}_{\mathrm{c}} \mathrm{H}_{\mathrm{h}} \mathrm{O}_{\mathrm{o}}$} & 0.20 & 1.10 & 0.70 & 0.30 & 0.10 & 2.30 & 0.10 & $2.16-2.44$ \\
\hline 2 & & 0.00 & 19.20 & 0.00 & 0.60 & 0.10 & 19.90 & 0.10 & $19.76-20.04$ \\
\hline 3 & & 0.10 & 2.10 & 0.10 & 0.70 & 0.10 & 3.00 & 0.40 & $2.45-3.55$ \\
\hline 4 & & 0.10 & 0.30 & 0.10 & 0.30 & 0.00 & 0.80 & 0.10 & $0.66-0.94$ \\
\hline 5 & & 0.00 & 0.50 & 0.00 & 0.60 & 0.00 & 1.20 & 0.20 & $0.92-1.48$ \\
\hline 1 & \multirow{5}{*}{$\mathrm{C}_{\mathrm{c}} \mathrm{H}_{\mathrm{h}} \mathrm{O}_{\mathrm{o}} \mathrm{S}_{\mathrm{s}}$} & 0.03 & 0.04 & 0.12 & 0.08 & 0.03 & 0.29 & 0.10 & $0.15-0.43$ \\
\hline 2 & & 0.00 & 0.02 & 0.02 & 0.08 & 0.00 & 0.11 & 0.00 & - \\
\hline 3 & & 0.08 & 0.04 & 0.01 & 0.07 & 0.04 & 0.24 & 0.10 & $0.10-0.38$ \\
\hline 4 & & 0.03 & 0.01 & 0.00 & 0.08 & 0.01 & 0.14 & 0.00 & - \\
\hline 5 & & 0.00 & 0.07 & 0.01 & 0.29 & 0.01 & 0.39 & 0.10 & $0.25-0.53$ \\
\hline 1 & \multirow{5}{*}{$\mathrm{C}_{\mathrm{c}} \mathrm{H}_{\mathrm{h}} \mathrm{N}_{\mathrm{n}} \mathrm{O}_{\mathrm{o}}$} & 0.20 & 12.10 & 0.20 & 1.20 & 0.70 & 14.40 & 1.40 & $12.46-16.34$ \\
\hline 2 & & 0.10 & 3.00 & 0.20 & 2.30 & 0.40 & 6.00 & 0.20 & $5.72-6.28$ \\
\hline 3 & & 0.10 & 13.20 & 0.20 & 2.60 & 0.30 & 16.30 & 0.90 & $15.05-17.8$ \\
\hline 4 & & 0.10 & 7.00 & 0.10 & 2.40 & 0.60 & 10.20 & 1.20 & $8.54-11.86$ \\
\hline 5 & & 0.10 & 5.50 & 0.10 & 2.70 & 0.20 & 8.60 & 0.20 & $8.32-8.88$ \\
\hline 1 & \multirow{5}{*}{$\mathrm{C}_{\mathrm{c}} \mathrm{H}_{\mathrm{h}} \mathrm{N}_{\mathrm{n}} \mathrm{O}_{\mathrm{o}} \mathrm{S}_{\mathrm{s}}$} & 0.10 & 0.80 & 0.70 & 0.90 & 0.10 & 2.60 & 0.10 & $2.46-2.74$ \\
\hline 2 & & 0.00 & 0.00 & 0.10 & 1.10 & 0.10 & 1.40 & 0.00 & - \\
\hline 3 & & 0.10 & 0.70 & 0.10 & 1.20 & 0.10 & 2.10 & 0.20 & $1.82-2.38$ \\
\hline 4 & & 0.10 & 0.60 & 0.00 & 0.80 & 0.10 & 1.50 & 0.10 & $1.36-1.64$ \\
\hline 5 & & 0.00 & 0.30 & 0.10 & 1.60 & 0.00 & 2.00 & 0.40 & $1.45-2.55$ \\
\hline
\end{tabular}

Table S5. The total $w t \%$ of nitrogen $(\mathrm{N})$, sulfur $(\mathrm{S})$ and oxygen $(\mathrm{O})$ containing compounds recovered from all fractions of the condensate like oils.

\begin{tabular}{lcccccccccc} 
& \multicolumn{8}{c}{ Approximate (consolidated) $\mathrm{wt} \%$ of compounds with heteroatom content } \\
\cline { 2 - 6 } & Trial 1 & Trial 2 & SD Average & $\begin{array}{c}95 \% \\
\text { Confidence } \\
\text { interval }\end{array}$ & Trial 1 & Trial 2 & SD & $\begin{array}{c}\text { Average } \\
95 \% \text { Confidence } \\
\text { interval }\end{array}$ \\
Total N content & 12.4 & 12.5 & 0.07 & 12.5 & $12.4-12.6$ & 14.1 & 14.0 & 0.1 & 14.1 & $14.0-14.2$ \\
Total S content & 3.9 & 3.9 & 0.0 & 3.9 & - & 3.8 & 3.9 & 0.1 & 3.9 & $3.8-4.0$ \\
Total O content & 13.1 & 13.0 & 0.07 & 13.1 & $13.0-13.2$ & 15.3 & 14.1 & 0.8 & 14.7 & $13.9-15.5$ \\
\hline
\end{tabular}

Table $\mathbf{S 5}$ continued

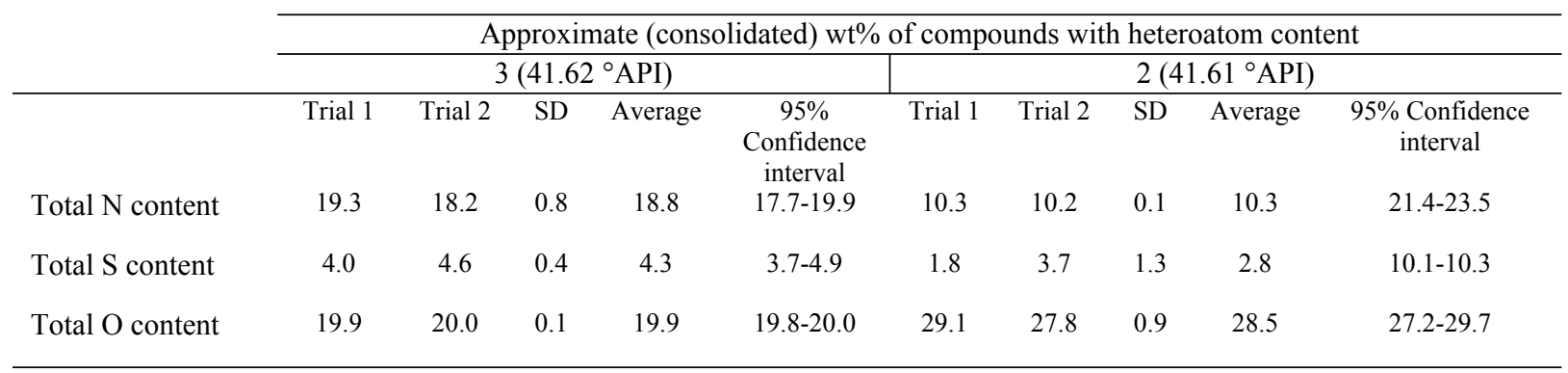


Table S5 continued

\begin{tabular}{lccccc}
\cline { 2 - 6 } & \multicolumn{5}{c}{$\begin{array}{c}\text { Approximate (consolidated) } \mathrm{wt} \% \text { of } \\
\text { compounds with heteroatom content }\end{array}$} \\
\cline { 2 - 6 } & Trial 1 & Trial 2 & SD & Average & $\begin{array}{c}95 \% \\
\text { Confidence } \\
\text { interval }\end{array}$ \\
& & & & & \\
Total N content & 20.5 & 18.1 & 1.7 & 19.3 & $16.9-21.7$ \\
Total S content & 5.4 & 6.3 & 0.6 & 5.9 & $5.0-6.8$ \\
Total O content & 24.2 & 20.1 & 2.9 & 22.2 & $18.2-26.2$ \\
\hline
\end{tabular}

Table S6. Elemental composition types of the compounds in the volatile saturated hydrocarbon class derived from five different condensate-like oils and the relative abundances of the ionized compounds based on mass spectrometry.

\begin{tabular}{|l|c|c|c|c|c|}
\hline & \multicolumn{5}{|c|}{ Relative Ion Abundances (\%) } \\
\hline Elemental Compositions & $5\left(43.00^{\circ} \mathrm{API}\right)$ & $4\left(42.4^{\circ} \mathrm{API}\right)$ & $3\left(41.62^{\circ} \mathrm{API}\right)$ & $2\left(41.61^{\circ} \mathrm{API}\right)$ & $1\left(37.50^{\circ} \mathrm{API}\right)$ \\
\hline $\mathrm{C}_{\mathrm{c}} \mathrm{H}_{\mathrm{h}}$ & 72.74 & 76.25 & 77.52 & 79.23 & 66.08 \\
$\mathrm{C}_{\mathrm{c}} \mathrm{H}_{\mathrm{h}} \mathrm{S}_{\mathrm{s}}$ & 0.96 & 6.68 & 2.14 & 3.73 & 0.91 \\
$\mathrm{C}_{\mathrm{c}} \mathrm{H}_{\mathrm{h}} \mathrm{N}_{\mathrm{n}}$ & 1.88 & 1.41 & 1.19 & 0.27 & 1.34 \\
$\mathrm{C}_{\mathrm{c}} \mathrm{H}_{\mathrm{h}} \mathrm{O}_{\mathrm{o}}$ & 4.31 & 5.14 & 7.79 & 2.4 & 8.4 \\
$\mathrm{C}_{\mathrm{c}} \mathrm{H}_{\mathrm{h}} \mathrm{O}_{\mathrm{o}} \mathrm{S}_{\mathrm{s}}$ & 0.41 & 2.16 & 4.00 & 0.21 & 1.32 \\
$\mathrm{C}_{\mathrm{c}} \mathrm{H}_{\mathrm{h}} \mathrm{N}_{\mathrm{n}} \mathrm{O}_{\mathrm{o}}$ & 9.96 & 3.32 & 4.27 & 6.32 & 9.44 \\
$\mathrm{C}_{\mathrm{c}} \mathrm{H}_{\mathrm{h}} \mathrm{N}_{\mathrm{n}} \mathrm{O}_{\mathrm{o}} \mathrm{S}_{\mathrm{s}}$ & 0.45 & 5.04 & 3.09 & 2.58 & 6.09 \\
Others & 9.30 & 0.00 & 0.00 & 5.26 & 6.42 \\
Total & 100.00 & 100.00 & 100.00 & 100.00 & 100.00 \\
\hline
\end{tabular}

Table S7. Elemental composition types of the compounds in the heavy saturated hydrocarbons class derived from five different condensate-like oils and the relative abundances of the ionized compounds based on mass spectrometry.

\begin{tabular}{|l|c|c|c|c|c|}
\hline & \multicolumn{5}{|c|}{ Relative Ion Abundances (\%) } \\
\hline Elemental Compositions & $5\left(43.00^{\circ} \mathrm{API}\right)$ & $4\left(42.4^{\circ} \mathrm{API}\right)$ & $3\left(41.62^{\circ} \mathrm{API}\right)$ & $2\left(41.61^{\circ} \mathrm{API}\right)$ & $1\left(37.50^{\circ} \mathrm{API}\right)$ \\
\hline $\mathrm{C}_{\mathrm{c}} \mathrm{H}_{\mathrm{h}}$ & 90.69 & 88.57 & 77.65 & 68.23 & 77.07 \\
$\mathrm{C}_{\mathrm{c}} \mathrm{H}_{\mathrm{h}} \mathrm{S}_{\mathrm{s}}$ & 0.00 & 0.00 & 0.04 & 0.00 & 0.04 \\
$\mathrm{C}_{\mathrm{c}} \mathrm{H}_{\mathrm{h}} \mathrm{N}_{\mathrm{n}}$ & 1.30 & 0.52 & 0.33 & 2.45 & 0.71 \\
$\mathrm{C}_{\mathrm{c}} \mathrm{H}_{\mathrm{h}} \mathrm{O}_{\mathrm{o}}$ & 0.60 & 0.44 & 2.74 & 25.17 & 1.62 \\
$\mathrm{C}_{\mathrm{c}} \mathrm{H}_{\mathrm{h}} \mathrm{O}_{\mathrm{o}} \mathrm{S}_{\mathrm{s}}$ & 0.08 & 0.02 & 0.05 & 0.02 & 0.06 \\
$\mathrm{C}_{\mathrm{c}} \mathrm{H}_{\mathrm{h}} \mathrm{N}_{\mathrm{n}} \mathrm{O}_{\mathrm{o}}$ & 6.71 & 8.93 & 17.39 & 3.95 & 17.53 \\
$\mathrm{C}_{\mathrm{c}} \mathrm{H}_{\mathrm{h}} \mathrm{N}_{\mathrm{n}} \mathrm{O}_{\mathrm{o}} \mathrm{S}_{\mathrm{s}}$ & 0.29 & 0.78 & 0.94 & 0.02 & 0.16 \\
Others & 0.32 & 0.74 & 0.87 & 100.00 & 2.14 \\
Total & 100.00 & 100.00 & 100.00 & 100.00 \\
\hline
\end{tabular}


Table S8. Elemental composition types of the compounds in the alkyl aromatic hydrocarbons class derived from five different condensate-like oils and the relative abundances of the ionized compounds based on mass spectrometry.

\begin{tabular}{|l|c|c|c|c|c|}
\hline & \multicolumn{5}{|c|}{ Relative Ion Abundances (\%) } \\
\hline Elemental Compositions & $5\left(43.00^{\circ} \mathrm{API}\right)$ & $4\left(42.4^{\circ} \mathrm{API}\right)$ & $3\left(41.62^{\circ} \mathrm{API}\right)$ & $2\left(41.61^{\circ} \mathrm{API}\right)$ & $1\left(37.50^{\circ} \mathrm{API}\right)$ \\
\hline $\mathrm{C}_{\mathrm{c}} \mathrm{H}_{\mathrm{h}}$ & 63.22 & 75.38 & 60.46 & 44.48 & 32.32 \\
$\mathrm{C}_{\mathrm{c}} \mathrm{H}_{\mathrm{h}} \mathrm{S}_{\mathrm{s}}$ & 1.03 & 0.81 & 1.40 & 1.00 & 0.02 \\
$\mathrm{C}_{\mathrm{c}} \mathrm{H}_{\mathrm{h}} \mathrm{N}_{\mathrm{n}}$ & 0.50 & 0.50 & 1.01 & 1.5 & 29.8 \\
$\mathrm{C}_{\mathrm{c}} \mathrm{H}_{\mathrm{h}} \mathrm{O}_{\mathrm{o}}$ & 3.10 & 8.80 & 6.54 & 1.27 & 3.41 \\
$\mathrm{C}_{\mathrm{c}} \mathrm{H}_{\mathrm{h}} \mathrm{O}_{\mathrm{o}} \mathrm{S}_{\mathrm{s}}$ & 2.38 & 0.57 & 1.24 & 2.00 & 3.28 \\
$\mathrm{C}_{\mathrm{c}} \mathrm{H}_{\mathrm{h}} \mathrm{N}_{\mathrm{n}} \mathrm{O}_{\mathrm{o}}$ & 11.76 & 9.90 & 19.70 & 31.82 & 13.81 \\
$\mathrm{C}_{\mathrm{c}} \mathrm{H}_{\mathrm{h}} \mathrm{N}_{\mathrm{n}} \mathrm{O}_{\mathrm{o}} \mathrm{S}_{\mathrm{s}}$ & 13.27 & 2.91 & 9.14 & 11.12 & 12 \\
Others & 4.74 & 1.14 & 0.50 & 6.81 & 10.96 \\
Total & 100.00 & 100.00 & 100.00 & & 6.4 \\
\hline
\end{tabular}

Table S9. Elemental composition types of the compounds in the heteroaromatic compound class derived from five different condensate-like oils and the relative abundances of the ionized compounds based on mass spectrometry.

\begin{tabular}{|l|c|c|c|c|c|}
\hline & \multicolumn{5}{|c|}{ Relative Ion Abundances (\%) } \\
\hline Elemental Compositions & $5\left(43.00^{\circ} \mathrm{API}\right)$ & $4\left(42.4^{\circ} \mathrm{API}\right)$ & $3\left(41.62^{\circ} \mathrm{API}\right)$ & $2\left(41.61^{\circ} \mathrm{API}\right)$ & $1\left(37.50^{\circ} \mathrm{API}\right)$ \\
\hline $\mathrm{C}_{\mathrm{c}} \mathrm{H}_{\mathrm{h}}$ & 17.56 & 39.72 & 46.14 & 41.77 & 74.29 \\
$\mathrm{C}_{\mathrm{c}} \mathrm{H}_{\mathrm{h}} \mathrm{S}_{\mathrm{s}}$ & 2.12 & 2.90 & 3.03 & 2.20 & 6.06 \\
$\mathrm{C}_{\mathrm{c}} \mathrm{H}_{\mathrm{h}} \mathrm{N}_{\mathrm{n}}$ & 2.07 & 2.45 & 2.17 & 2.26 & 0.33 \\
$\mathrm{C}_{\mathrm{c}} \mathrm{H}_{\mathrm{h}} \mathrm{O}_{\mathrm{o}}$ & 8.75 & 5.18 & 6.29 & 6.89 & 2.22 \\
$\mathrm{C}_{\mathrm{c}} \mathrm{H}_{\mathrm{h}} \mathrm{O}_{\mathrm{o}} \mathrm{S}_{\mathrm{s}}$ & 4.04 & 1.22 & 0.68 & 0.90 & 0.59 \\
$\mathrm{C}_{\mathrm{c}} \mathrm{H}_{\mathrm{h}} \mathrm{N}_{\mathrm{n}} \mathrm{O}_{\mathrm{o}}$ & 37.51 & 36.52 & 24.74 & 27.90 & 8.59 \\
$\mathrm{C}_{\mathrm{c}} \mathrm{H}_{\mathrm{h}} \mathrm{N}_{\mathrm{n}} \mathrm{O}_{\mathrm{o}} \mathrm{S}_{\mathrm{s}}$ & 22.01 & 11.94 & 1.15 & 1.12 & 6.38 \\
Others & 5.93 & 0.06 & 15.80 & 16.97 & 1.55 \\
Total & 100.00 & 100.00 & 100.00 & 100.00 & 100.00 \\
\hline
\end{tabular}

Table S10. Elemental composition types of the compounds in polar compounds class derived from five different condensate-like oils and the relative abundances of the ionized compounds based on mass spectrometry.

\begin{tabular}{|l|c|c|c|c|c|}
\hline & \multicolumn{5}{|c|}{ Relative Ion Abundances (\%) } \\
\hline Elemental Compositions & $5\left(43.00^{\circ} \mathrm{API}\right)$ & $4\left(42.4^{\circ} \mathrm{API}\right)$ & $3\left(41.62^{\circ} \mathrm{API}\right)$ & $2\left(41.61^{\circ} \mathrm{API}\right)$ & $1\left(37.50^{\circ} \mathrm{API}\right)$ \\
\hline $\mathrm{C}_{\mathrm{c}} \mathrm{H}_{\mathrm{h}}$ & 16.40 & 10.68 & 17.18 & 15.11 & 7.73 \\
$\mathrm{C}_{\mathrm{c}} \mathrm{H}_{\mathrm{h}} \mathrm{S}_{\mathrm{s}}$ & 0.88 & 3.36 & 0.75 & 1.61 & 1.15 \\
$\mathrm{C}_{\mathrm{c}} \mathrm{H}_{\mathrm{h}} \mathrm{N}_{\mathrm{n}}$ & 9.17 & 5.12 & 31.17 & 22.08 & 2.90 \\
$\mathrm{C}_{\mathrm{c}} \mathrm{H}_{\mathrm{h}} \mathrm{O}_{\mathrm{o}}$ & 7.06 & 2.95 & 6.26 & 6.31 & 3.47 \\
$\mathrm{C}_{\mathrm{c}} \mathrm{H}_{\mathrm{h}} \mathrm{O}_{\mathrm{o}} \mathrm{S}_{\mathrm{s}}$ & 2.22 & 1.02 & 4.48 & 0.00 & 1.47 \\
$\mathrm{C}_{\mathrm{c}} \mathrm{H}_{\mathrm{h}} \mathrm{N}_{\mathrm{n}} \mathrm{O}_{\mathrm{o}}$ & 44.40 & 49.09 & 37.01 & 43.80 & 41.88
\end{tabular}




\begin{tabular}{|l|c|c|c|c|c|}
$\mathrm{C}_{\mathrm{c}} \mathrm{H}_{\mathrm{h}} \mathrm{N}_{\mathrm{n}} \mathrm{O}_{\mathrm{o}} \mathrm{S}_{\mathrm{s}}$ & 6.95 & 4.12 & 0.08 & 0.08 & 2.84 \\
Others & 12.92 & 23.66 & 3.08 & 11.01 & 38.54 \\
Total & 100.00 & 100.00 & 100.00 & 100.00 & 100.00 \\
\hline
\end{tabular}

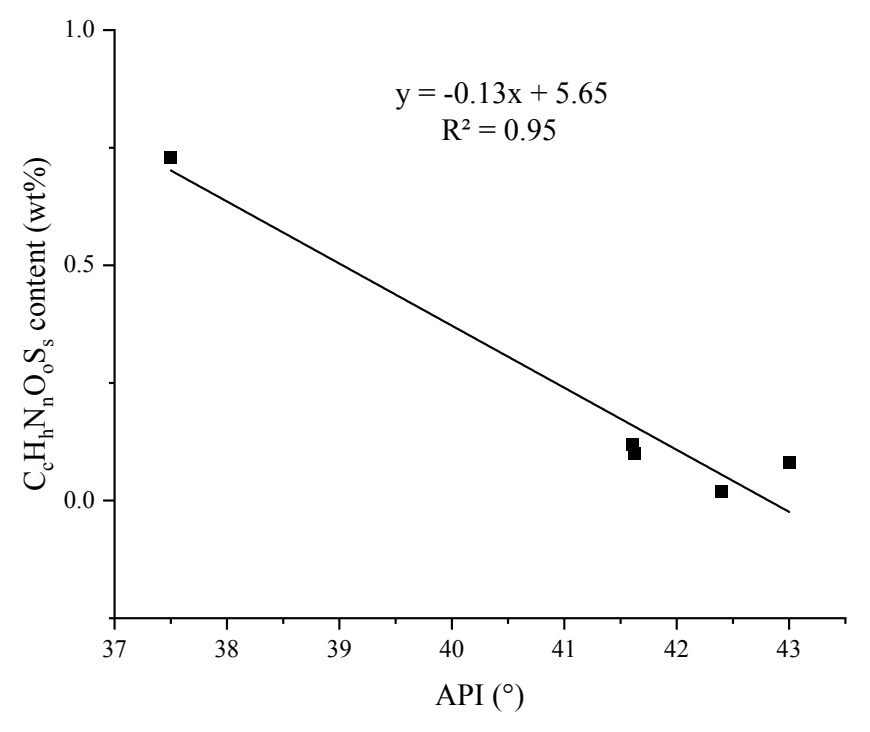

Figure S11. The average weight percent of compounds with the elemental composition type $\mathrm{C}_{c} \mathrm{H}_{\mathrm{h}} \mathrm{N}_{\mathrm{n}} \mathrm{O}_{\mathrm{o}} \mathrm{S}_{\mathrm{s}}$ in each heteroaromatic compound class as a function of API gravity for five different condensate-like oils. As the API gravity increases, the approximate average content of compounds with the elemental composition type $\mathrm{C}_{\mathrm{c}} \mathrm{H}_{\mathrm{h}} \mathrm{N}_{\mathrm{n}} \mathrm{O}_{\mathrm{o}} \mathrm{S}_{\mathrm{s}}$ in the heteroaromatic compound class decreases.

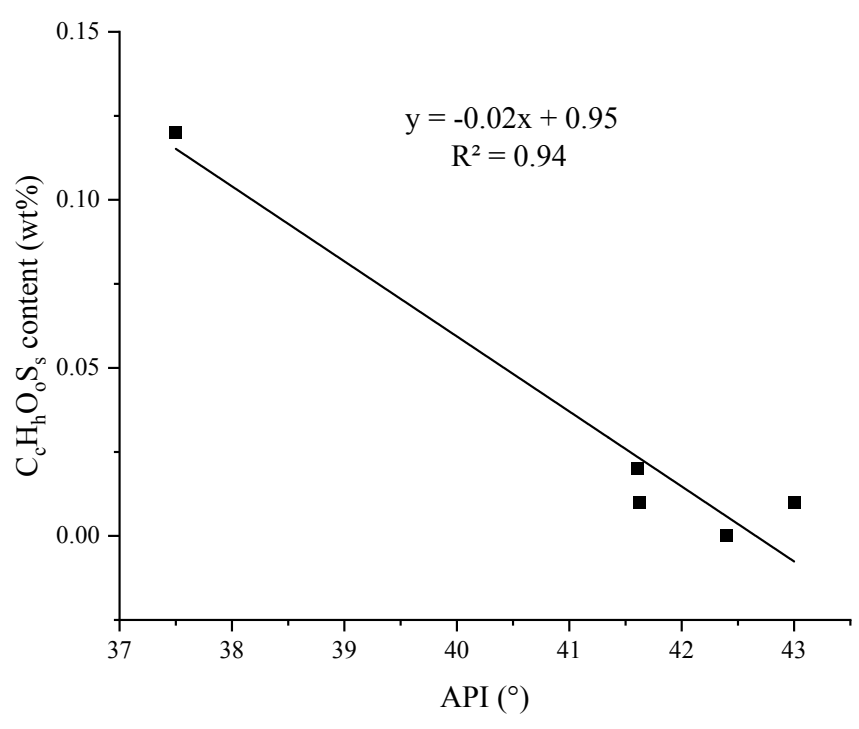

Figure S12. The average weight percent of compounds with the elemental composition type $\mathrm{C}_{\mathrm{c}} \mathrm{H}_{\mathrm{h}} \mathrm{O}_{\mathrm{o}} \mathrm{S}_{\mathrm{s}}$ in each heteroaromatic compound class as a function of API gravity for five different condensate-like oils: as the API gravity increases, the approximate average amount of compounds with the elemental composition type $\mathrm{C}_{\mathrm{c}} \mathrm{H}_{\mathrm{h}} \mathrm{O}_{\mathrm{o}} \mathrm{S}_{\mathrm{s}}$ in the heteroaromatic compound class decreases. 


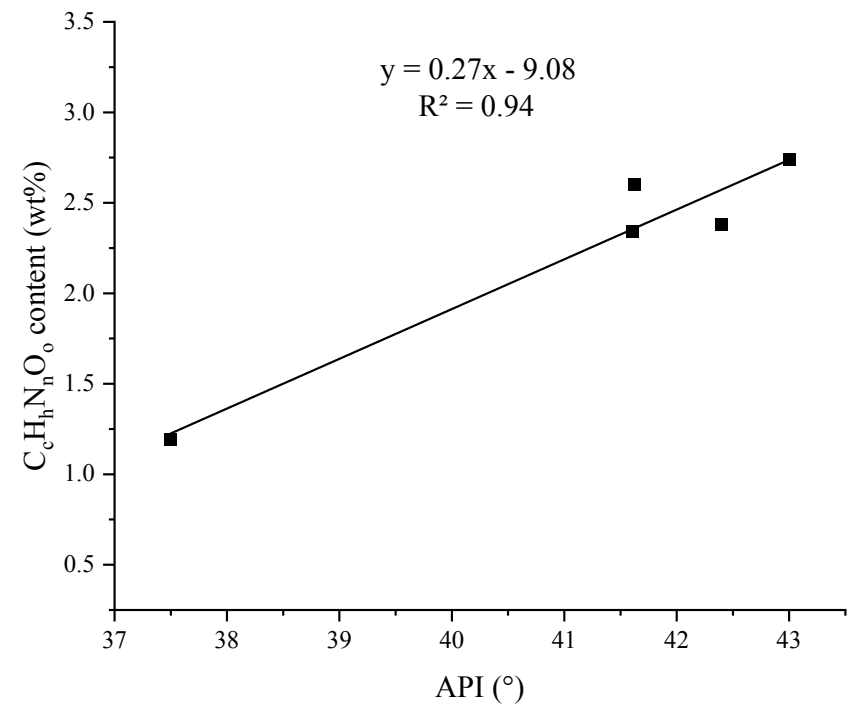

Figure S13. The average weight percent of compounds with the elemental composition type $\mathrm{C}_{c} \mathrm{H}_{h} \mathrm{~N}_{n} \mathrm{O}_{\mathrm{o}}$ in each alkyl aromatic compound class as a function of API gravity for five different condensate-like oils.

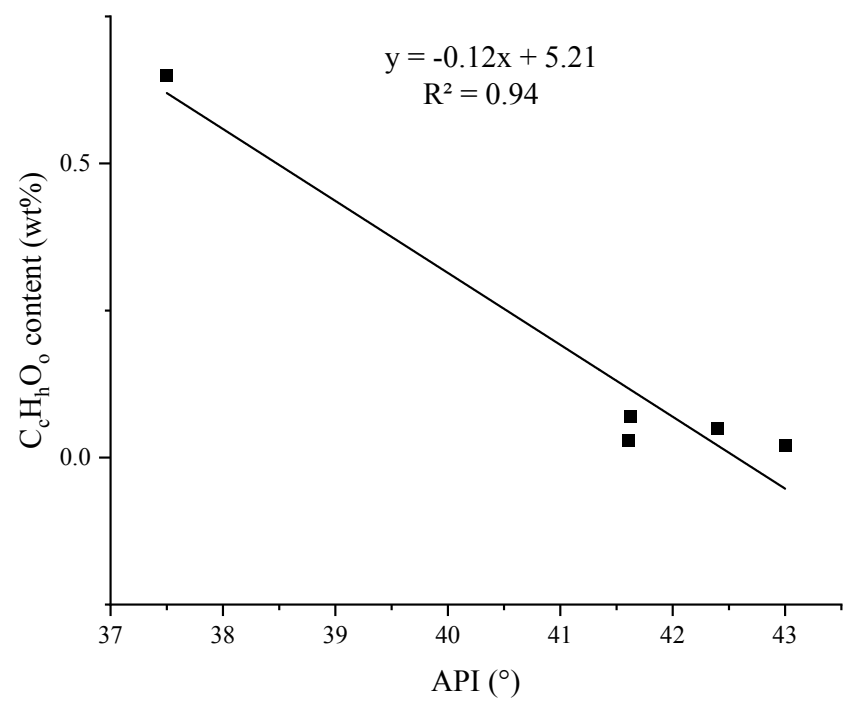

Figure S14. The average weight percent of compounds with the elemental composition type $\mathrm{C}_{c} \mathrm{H}_{\mathrm{h}} \mathrm{O}_{\mathrm{o}}$ in each heteroaromatic compound class as a function of API gravity for five different condensate-like oils. 


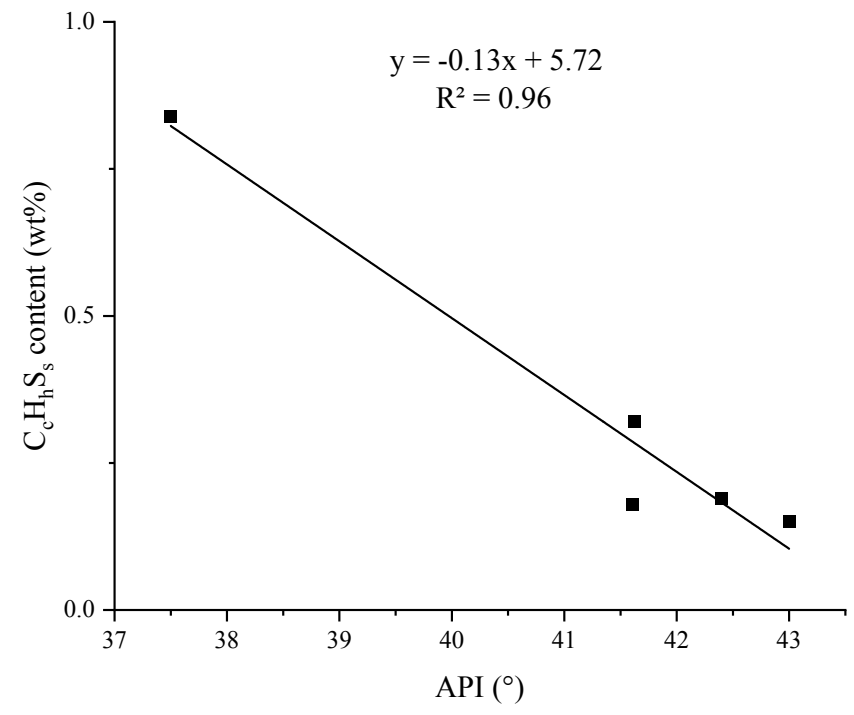

Figure S15. The average weight percent of compounds with the elemental composition type $\mathrm{C}_{\mathrm{c}} \mathrm{H}_{\mathrm{h}} \mathrm{S}_{\mathrm{s}}$ in each alkyl aromatic hydrocarbon class as a function of API gravity of five different condensate-like oils.

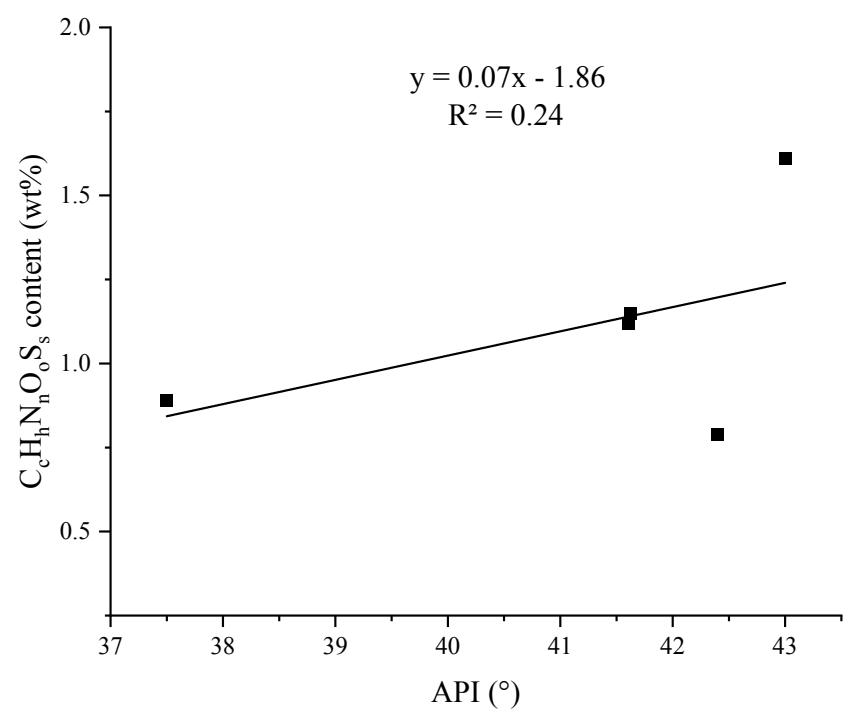

Figure S16. The average weight percent of compounds with the elemental composition type $\mathrm{C}_{c} \mathrm{H}_{h} \mathrm{~N}_{n} \mathrm{O}_{\mathrm{o}} \mathrm{S}_{\mathrm{s}}$ in each alkyl aromatic compound class as a function of API gravity of five different condensate-like oils. 


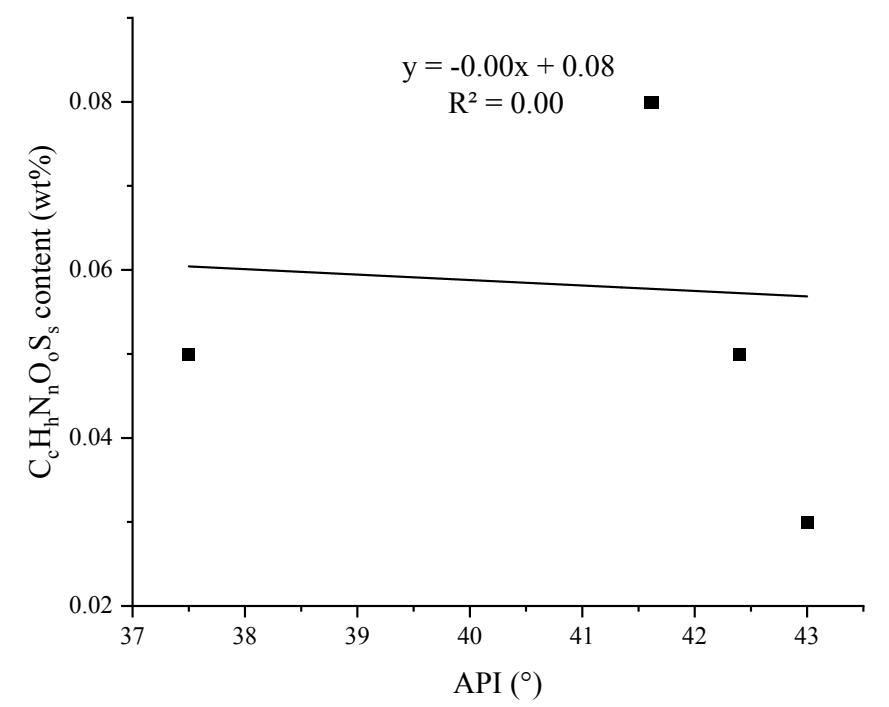

Figure S17. The average weight percent of compounds with the elemental composition type $\mathrm{C}_{c} \mathrm{H}_{h} \mathrm{~N}_{n} \mathrm{O}_{\mathrm{o}} \mathrm{S}_{\mathrm{s}}$ in each polar compound class as a function of API gravity of five different condensate-like oils.

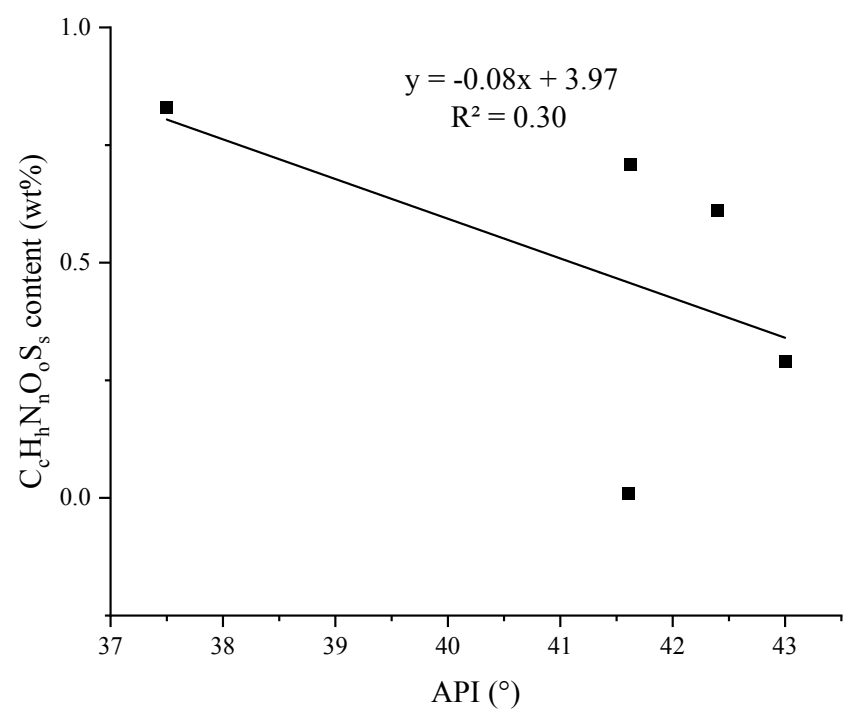

Figure S18. The average weight percent of compounds with the elemental composition type $\mathrm{C}_{c} \mathrm{H}_{h} \mathrm{~N}_{n} \mathrm{O}_{0} \mathrm{~S}_{\mathrm{s}}$ in each heavy saturated hydrocarbon class as a function of API gravity of five different condensate-like oils. 


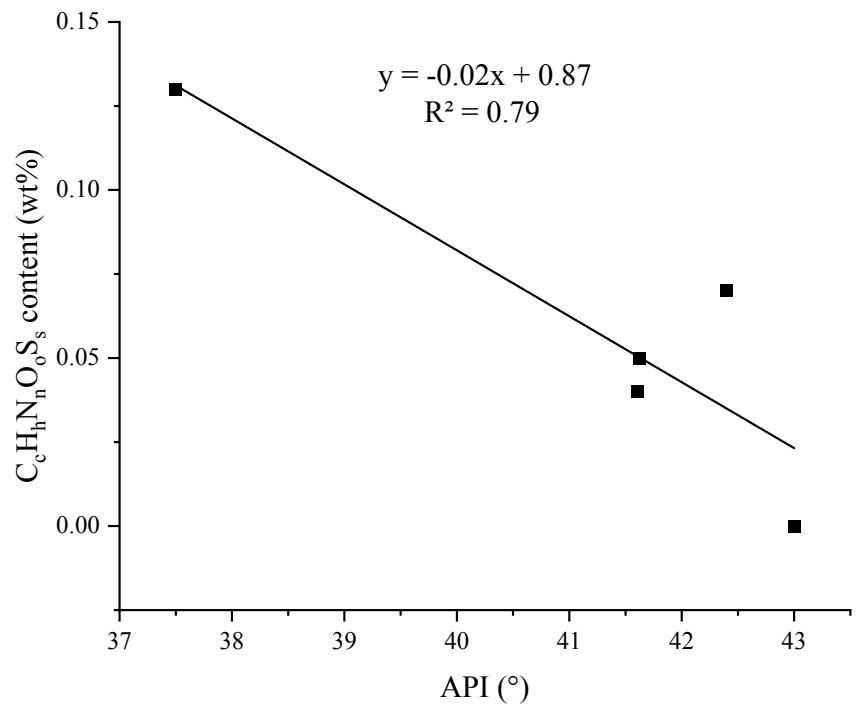

Figure S19. The average weight percent of compounds with the elemental composition type $\mathrm{C}_{\mathrm{c}} \mathrm{H}_{\mathrm{h}} \mathrm{N}_{\mathrm{n}} \mathrm{O}_{\mathrm{o}} \mathrm{S}_{\mathrm{s}}$ in each volatile hydrocarbon class as a function of API gravity of five different condensate-like oils.

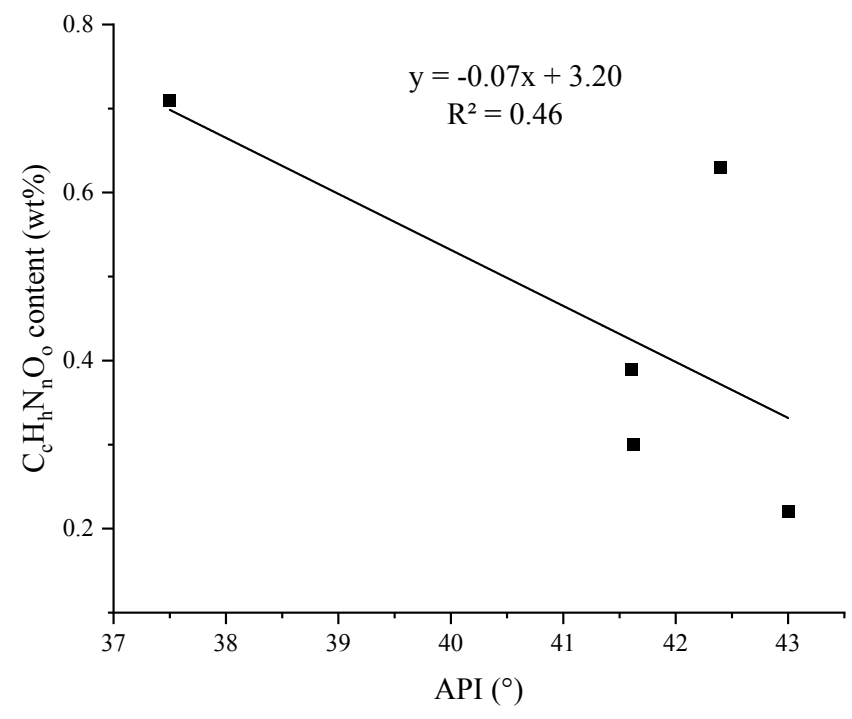

Figure S20. The average weight percent of compounds with the elemental composition type $\mathrm{C}_{c} \mathrm{H}_{h} \mathrm{~N}_{n} \mathrm{O}_{\mathrm{o}}$ in each polar compound class as a function of API gravity of five different condensate-like oils. 


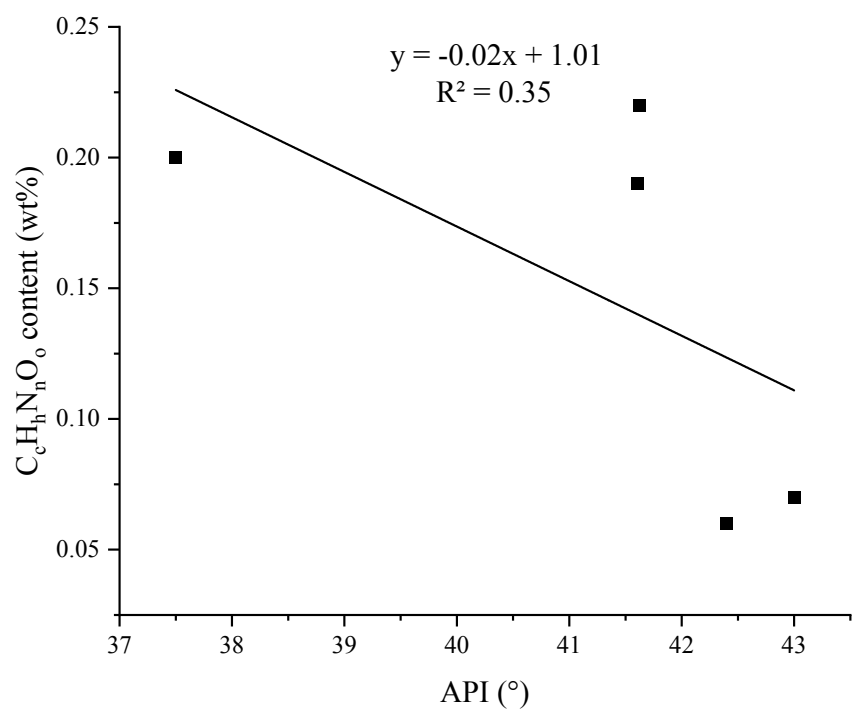

Figure S21. The average weight percent of compounds with the elemental composition type $\mathrm{C}_{c} \mathrm{H}_{h} \mathrm{~N}_{n} \mathrm{O}_{\mathrm{o}}$ in each heteroaromatic compound class as a function of API gravity of five different condensate-like oils.

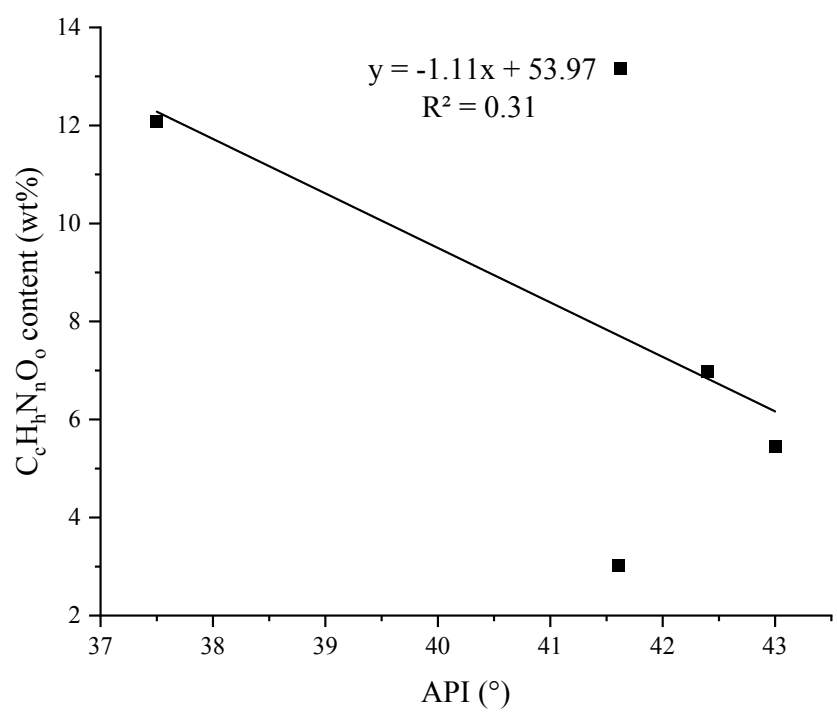

Figure S22. The average weight percent of compounds with the elemental composition type $\mathrm{C}_{c} \mathrm{H}_{\mathrm{h}} \mathrm{N}_{\mathrm{n}} \mathrm{O}_{\mathrm{o}}$ in each saturated hydrocarbon class as a function of API gravity of five different condensate-like oils. 


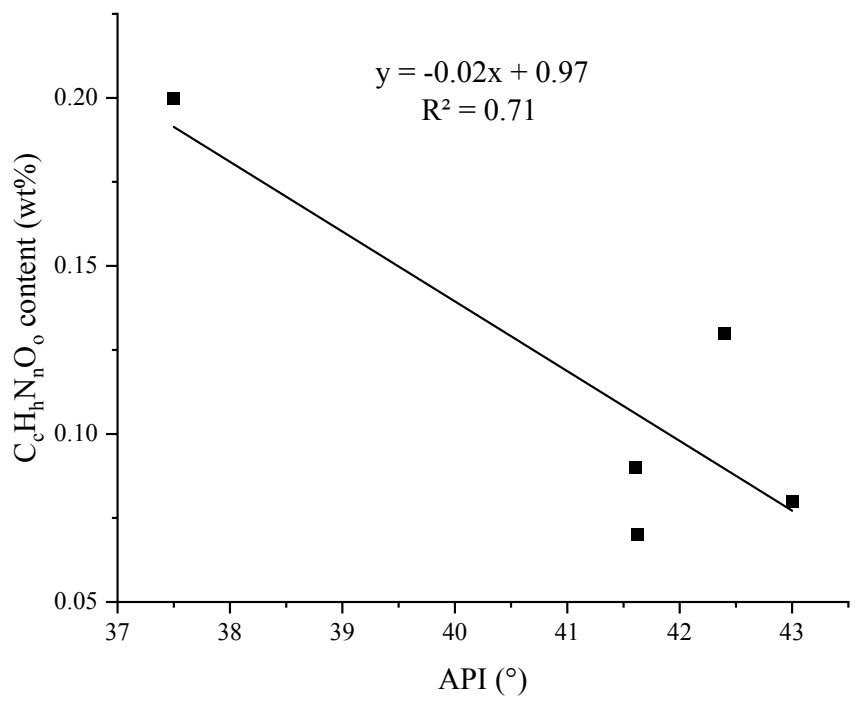

Figure S23. The average weight percent of compounds with the elemental composition type $\mathrm{C}_{c} \mathrm{H}_{h} \mathrm{~N}_{n} \mathrm{O}_{o}$ in each volatile hydrocarbon class as a function of API gravity of five different condensate-like oils.

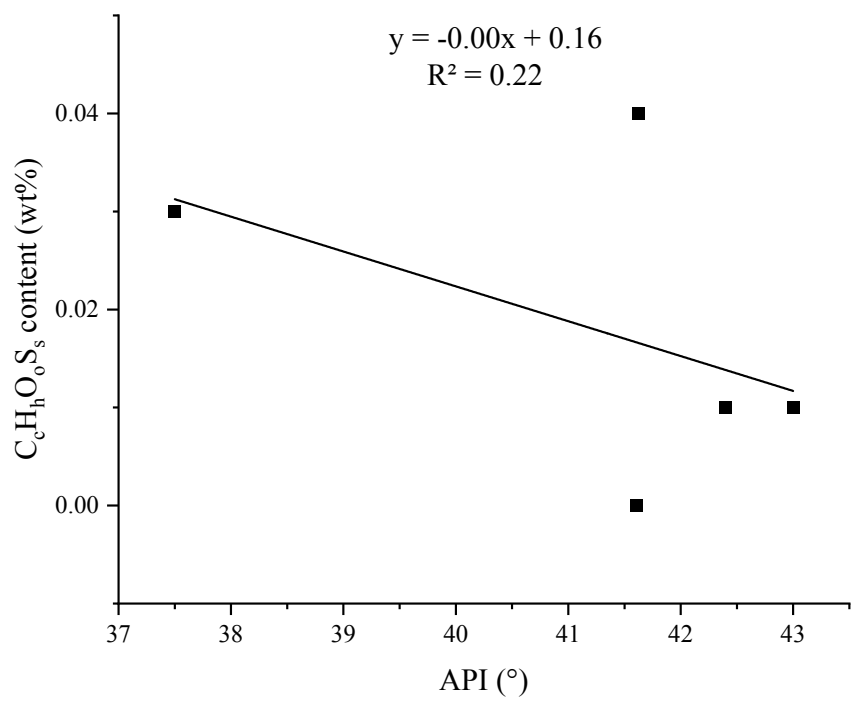

Figure 1S24. The average weight percent of compounds with the elemental composition type $\mathrm{C}_{c} \mathrm{H}_{\mathrm{h}} \mathrm{O}_{\mathrm{o}} \mathrm{S}_{\mathrm{s}}$ in each polar compound class as a function of API gravity of five different condensate-like oils. 


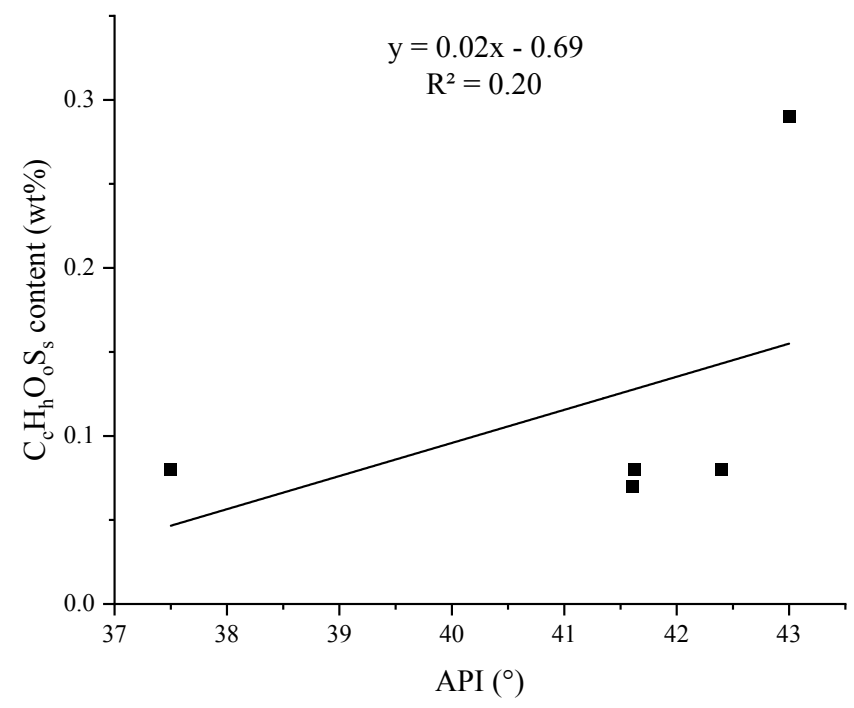

Figure 2S25. The average weight percent of compounds with the elemental composition type $\mathrm{C}_{\mathrm{c}} \mathrm{H}_{\mathrm{h}} \mathrm{O}_{\mathrm{o}} \mathrm{S}_{\mathrm{s}}$ in each alkyl aromatic hydrocarbon class as a function of API gravity of five different condensate-like oils.

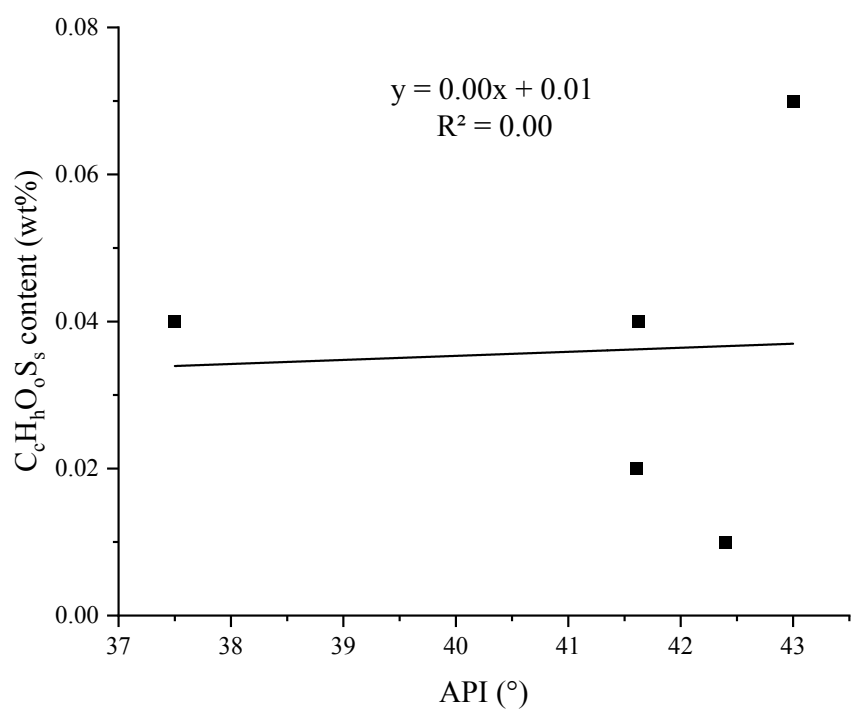

Figure S26. The average weight percent of compounds with the elemental composition type $\mathrm{C}_{c} \mathrm{H}_{h} \mathrm{O}_{\mathrm{o}} \mathrm{S}_{\mathrm{s}}$ in each heavy saturated hydrocarbon class as a function of API gravity of five different condensate-like oils. 


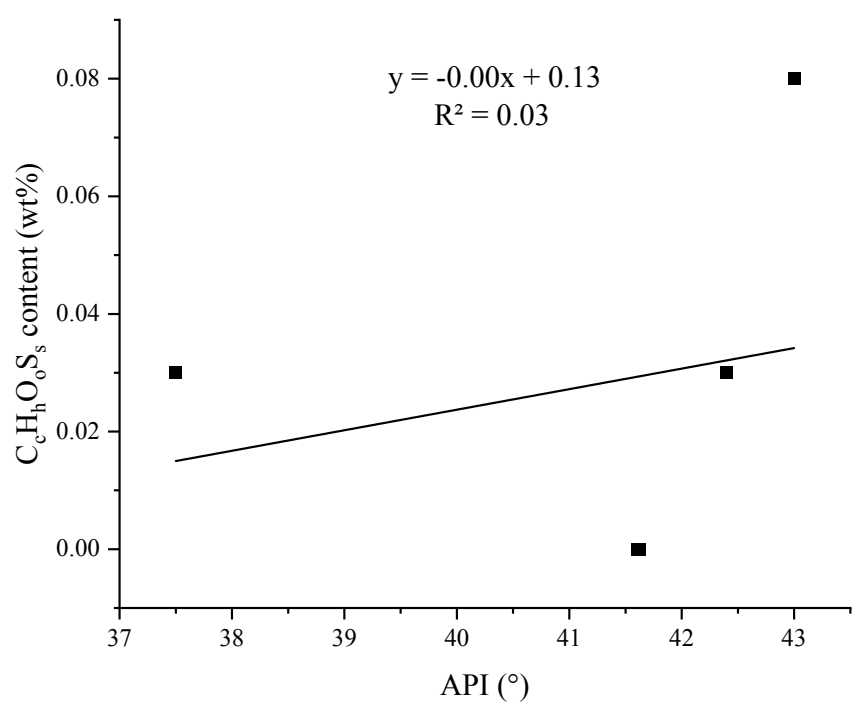

Figure S27. The average weight percent of compounds with the elemental composition type $\mathrm{C}_{c} \mathrm{H}_{\mathrm{h}} \mathrm{O}_{\mathrm{o}} \mathrm{S}_{\mathrm{s}}$ in each volatile hydrocarbon class as a function of API gravity of five different condensate-like oils.

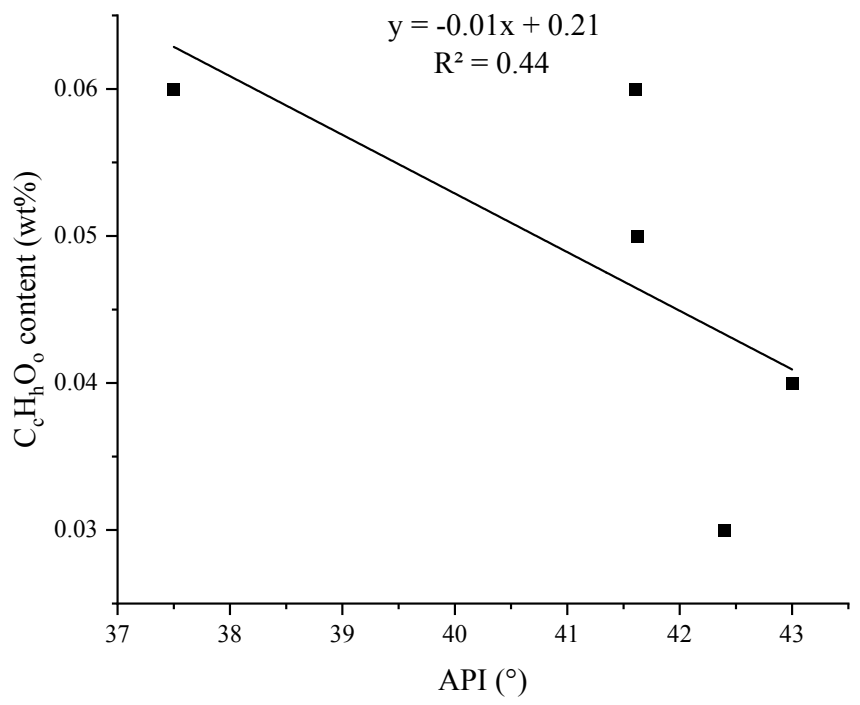

Figure S28. The average weight percent of compounds with the elemental composition type $\mathrm{C}_{\mathrm{c}} \mathrm{H}_{\mathrm{h}} \mathrm{O}_{\mathrm{o}}$ in each polar compound class as a function of API gravity of five different condensate-like oils. 


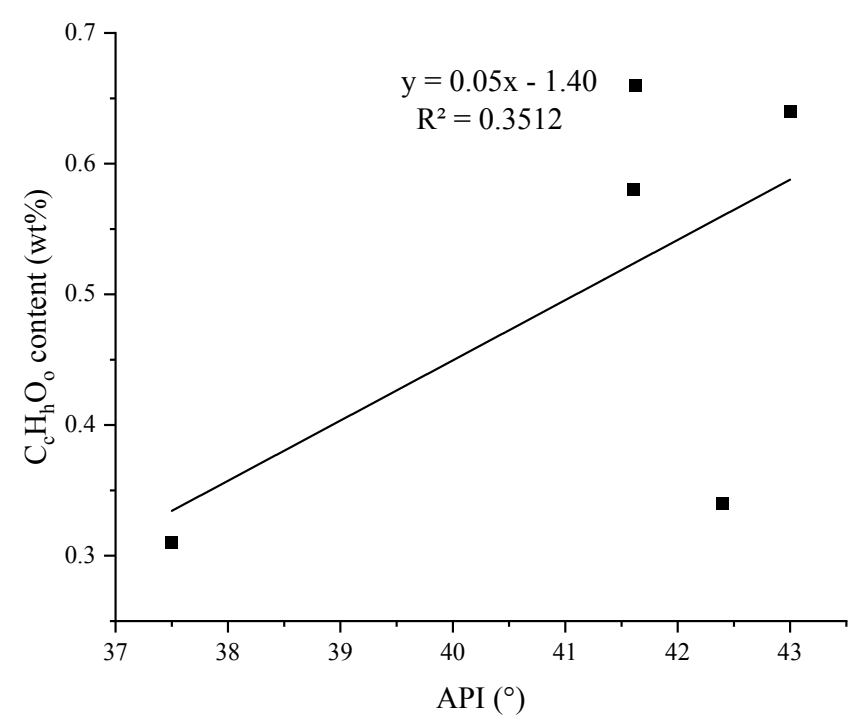

Figure S29. The average weight percent of compounds with the elemental composition type $\mathrm{C}_{\mathrm{c}} \mathrm{H}_{\mathrm{h}} \mathrm{O}_{\mathrm{o}}$ in each alkyl aromatic hydrocarbon class as a function of API gravity of five different condensate-like oils.

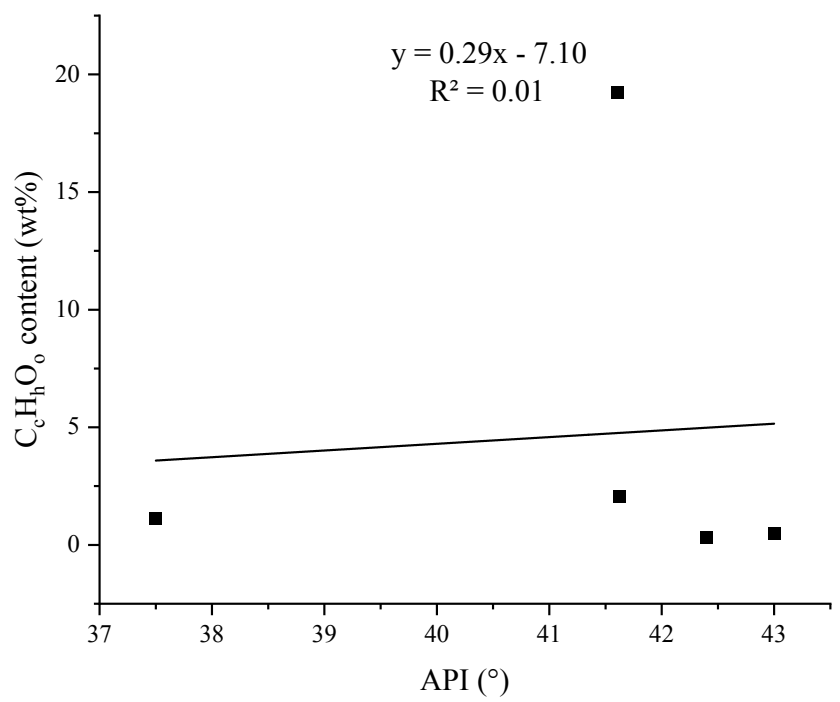

Figure S30. The average weight percent of compounds with the elemental composition type $\mathrm{C}_{c} \mathrm{H}_{h} \mathrm{O}_{\mathrm{o}}$ in each heavy saturated hydrocarbon class as a function of API gravity of five different condensate-like oils. 


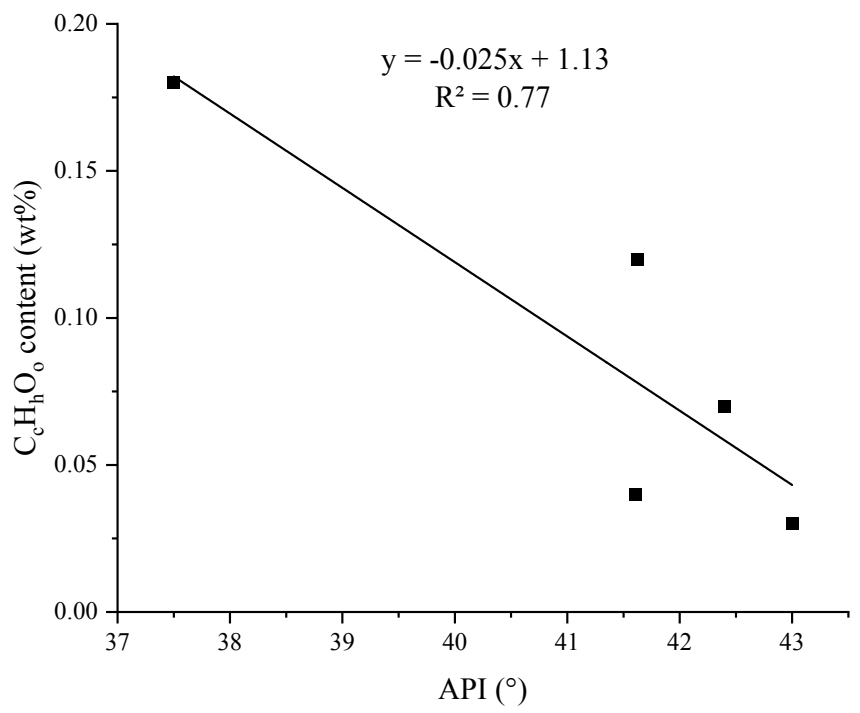

Figure S31. The average weight percent of compounds with the elemental composition type $\mathrm{C}_{c} \mathrm{H}_{h} \mathrm{O}_{\mathrm{o}}$ in each volatile hydrocarbon class as a function of API gravity of five different condensate-like oils.

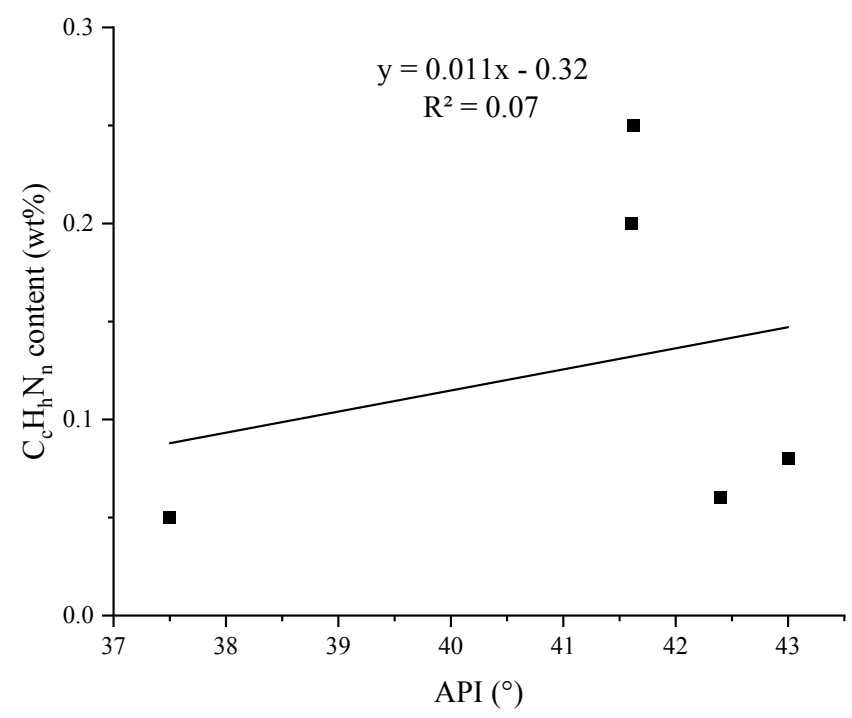

Figure S32. The average weight percent of compounds with the elemental composition type $\mathrm{C}_{c} \mathrm{H}_{\mathrm{h}} \mathrm{N}_{\mathrm{n}}$ in each polar compound class as a function of API gravity of five different condensate-like oils. 


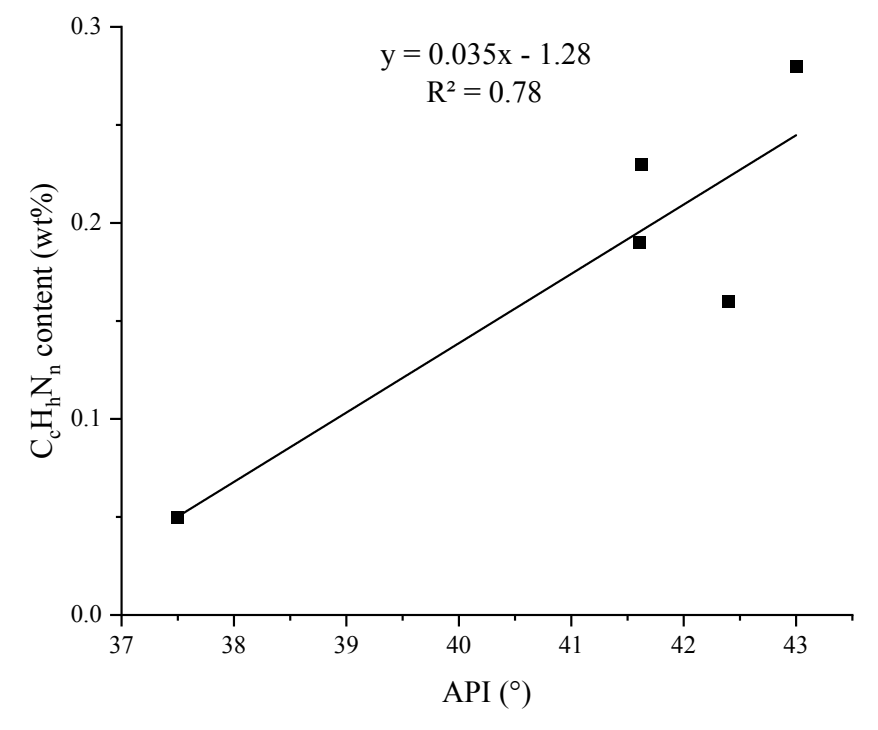

Figure S33. The average weight percent of compounds with the elemental composition type $\mathrm{C}_{c} \mathrm{H}_{\mathrm{h}} \mathrm{N}_{\mathrm{n}}$ in each alkyl aromatic hydrocarbon class as a function of API gravity of five different condensate-like oils.

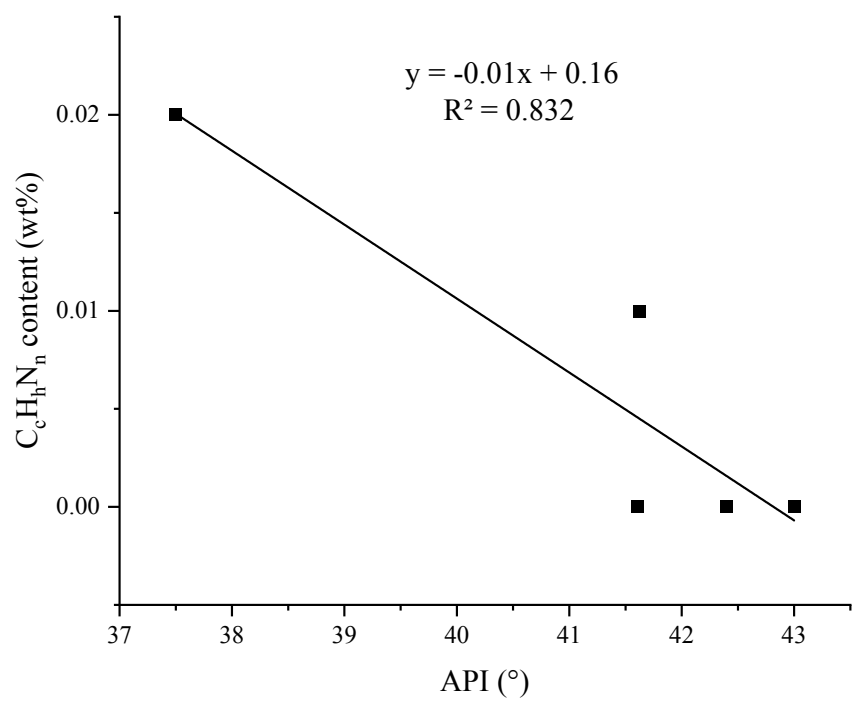

Figure S34. The average weight percent of compounds with the elemental composition type $\mathrm{C}_{c} \mathrm{H}_{\mathrm{h}} \mathrm{N}_{\mathrm{n}}$ in each heteroaromatic compound class as a function of API gravity of five different condensate-like oils. 


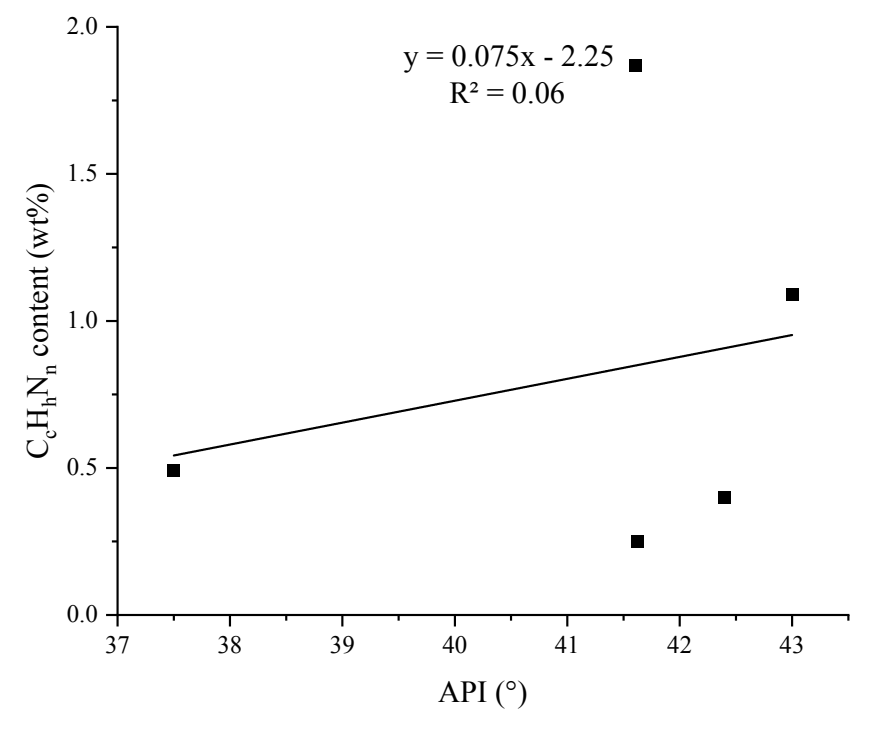

Figure S35. The average weight percent of compounds with the elemental composition type $\mathrm{C}_{c} \mathrm{H}_{h} \mathrm{~N}_{n}$ in each heavy saturated hydrocarbon class as a function of API gravity of five different condensate-like oils.

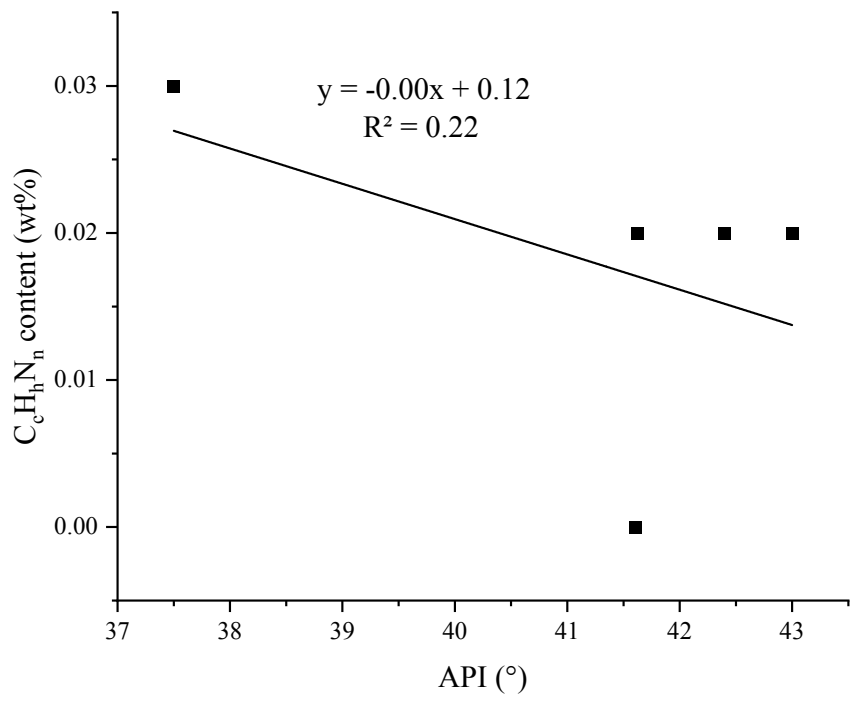

Figure S36. The average weight percent of compounds with the elemental composition type $\mathrm{C}_{c} \mathrm{H}_{h} \mathrm{~N}_{n}$ in each volatile hydrocarbon class as a function of API gravity of five different condensate-like oils. 


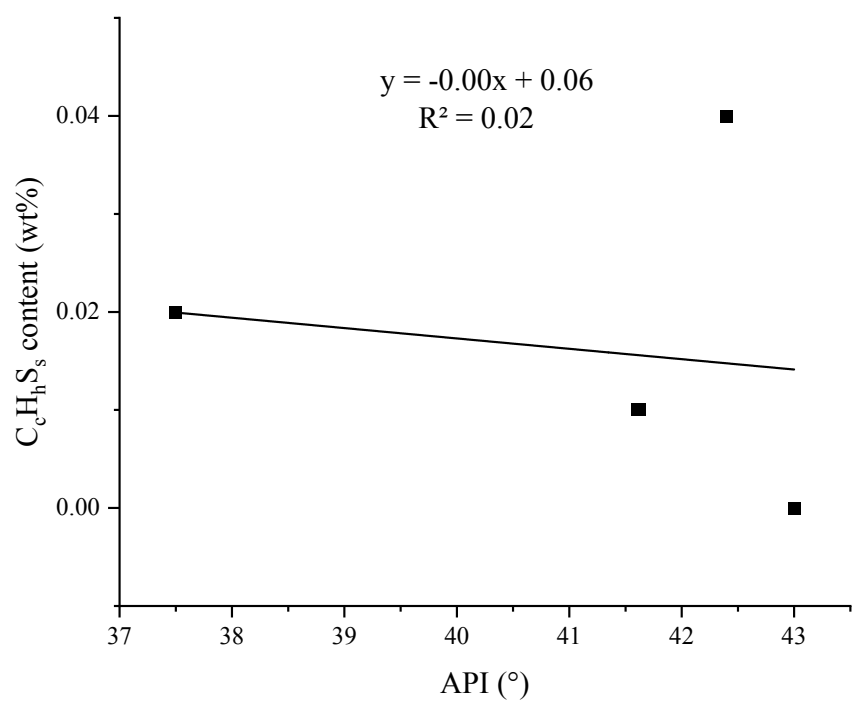

Figure S37. The average weight percent of compounds with the elemental composition type $\mathrm{C}_{\mathrm{c}} \mathrm{H}_{\mathrm{h}} \mathrm{S}_{\mathrm{s}}$ in each polar compound class as a function of API gravity of five different condensate-like oils.

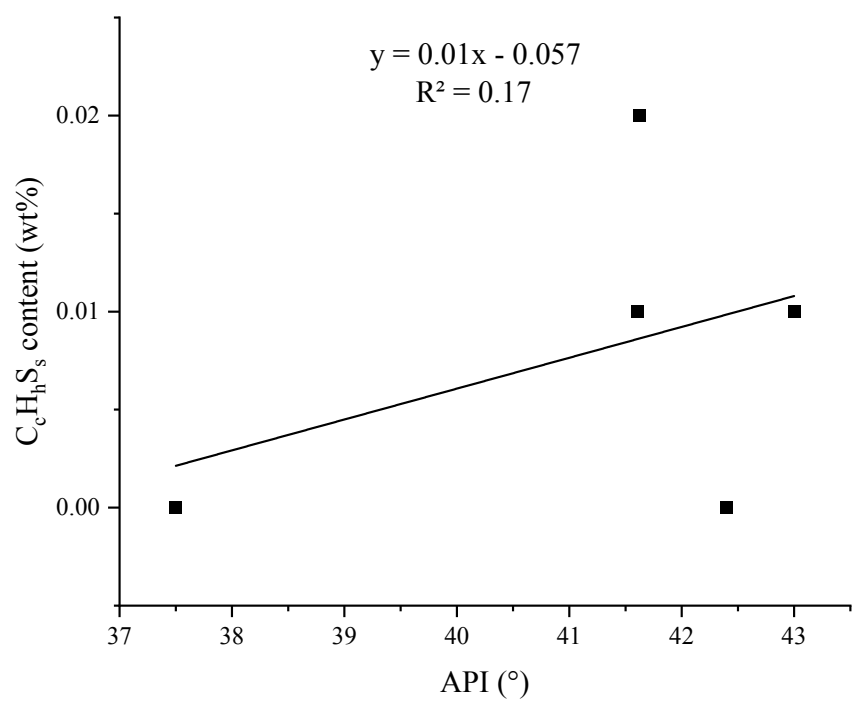

Figure S38. The average weight percent of compounds with the elemental composition type $\mathrm{C}_{\mathrm{c}} \mathrm{H}_{\mathrm{h}} \mathrm{S}_{\mathrm{s}}$ in each heteroaromatic compound class as a function of API gravity of five different condensate-like oils. 


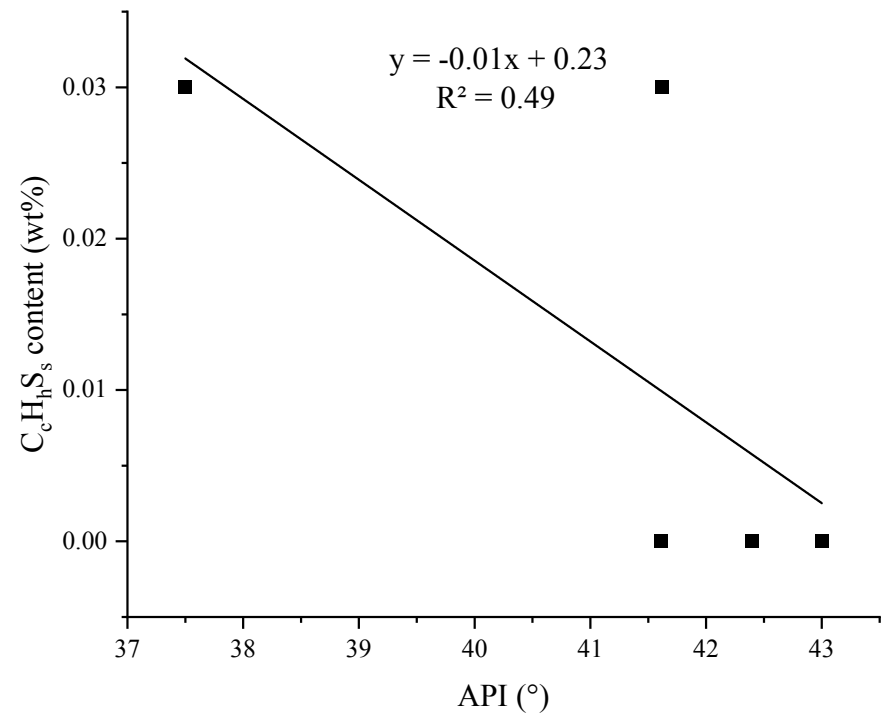

Figure S39. The average weight percent of compounds with the elemental composition type $\mathrm{C}_{\mathrm{c}} \mathrm{H}_{\mathrm{h}} \mathrm{S}_{\mathrm{s}}$ in each heavy saturated hydrocarbon class as a function of API gravity of five different condensate-like oils.

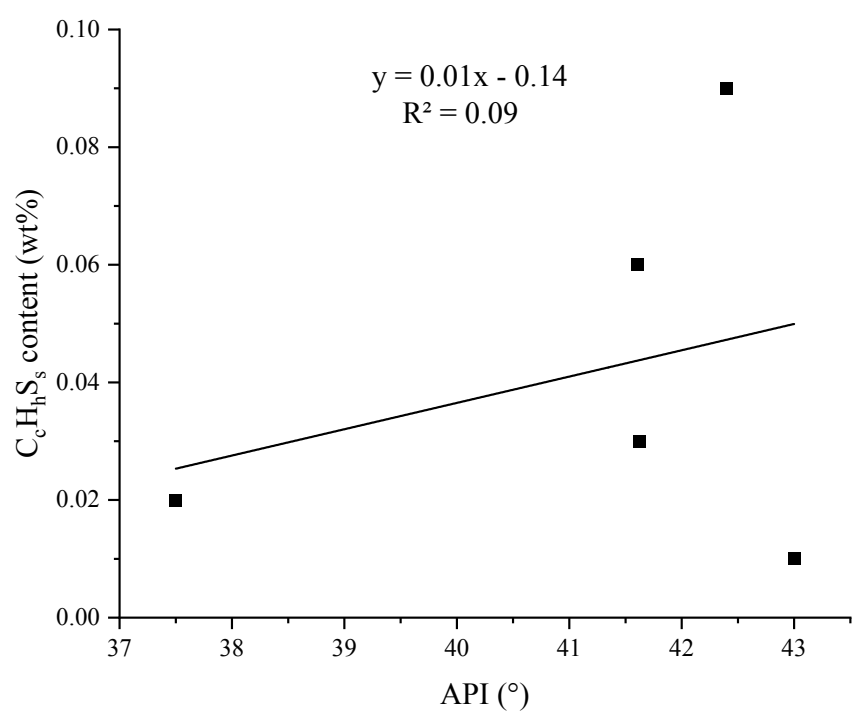

Figure S40. The average weight percent of compounds with the elemental composition type $\mathrm{C}_{\mathrm{c}} \mathrm{H}_{\mathrm{h}} \mathrm{S}_{\mathrm{s}}$ in each volatile hydrocarbon class as a function of API gravity of five different condensate-like oils. 


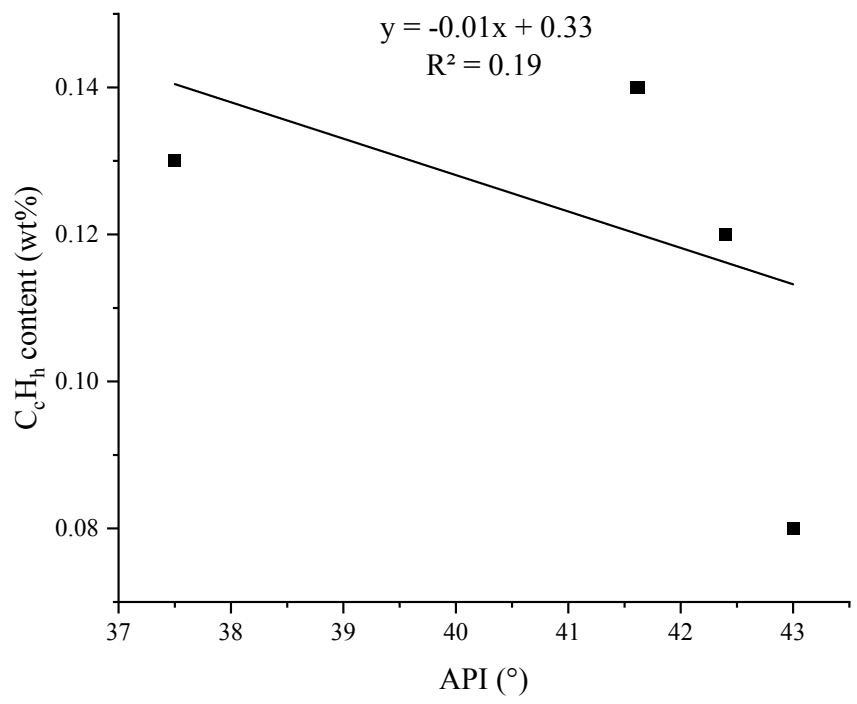

Figure S41. The average weight percent of compounds with the elemental composition type $\mathrm{C}_{\mathrm{c}} \mathrm{H}_{\mathrm{h}}$ in each polar compound class as a function of API gravity of five different condensate-like oils.

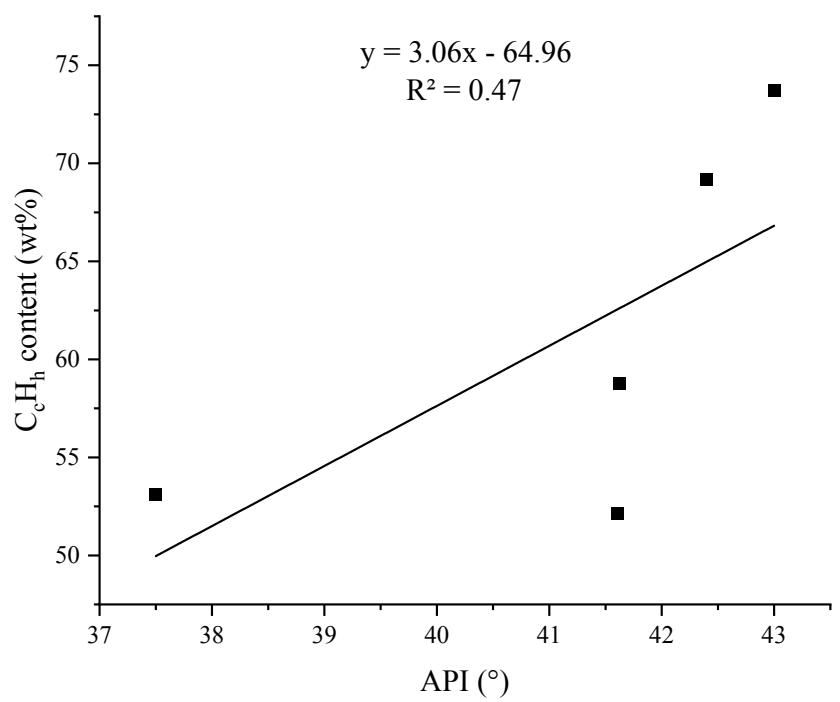

Figure S42. The average weight percent of compounds with the elemental composition type $\mathrm{C}_{\mathrm{c}} \mathrm{H}_{\mathrm{h}}$ in each heavy saturated hydrocarbon class as a function of API gravity of five different condensate-like oils. 


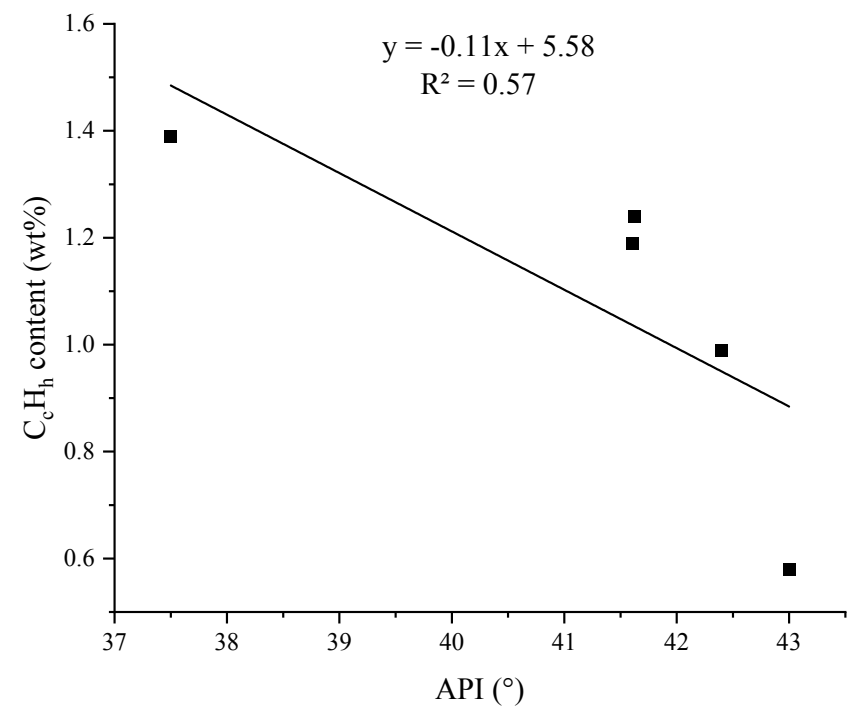

Figure S43. The average weight percent of compounds with the elemental composition type $\mathrm{C}_{\mathrm{c}} \mathrm{H}_{\mathrm{h}}$ in each volatile hydrocarbon class as a function of API gravity of five different condensate-like oils.

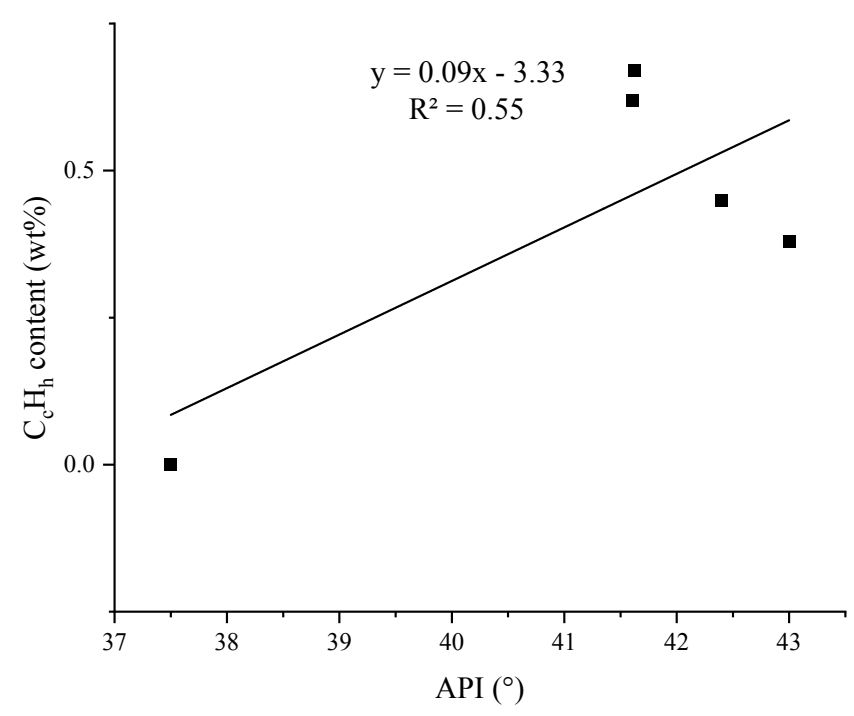

Figure S44. The average weight percent of compounds with the elemental composition type $\mathrm{C}_{\mathrm{c}} \mathrm{H}_{\mathrm{h}}$ in heteroaromatic compound class as a function of API gravity of five different condensate-like oils. 
Table S11. The average molecular weights of compounds in the different compound classes and their standard deviations (SD; based on two measurements) for five different condensate-like oils. The average molecular weights were determined based on the molecular weights determined for compounds in each individual compound class and the gravimetric weight percentage of each class.

\begin{tabular}{|c|c|c|c|c|c|c|c|c|c|c|}
\hline \multirow[b]{3}{*}{ Chemical Classes } & \multicolumn{9}{|c|}{ Average molecular weight (Da) } & \multirow{3}{*}{$\begin{array}{c} \\
95 \% \\
\text { Confidence } \\
\text { interval }\end{array}$} \\
\hline & \multicolumn{5}{|c|}{$5\left(43.00^{\circ} \mathrm{API}\right)$} & \multicolumn{4}{|c|}{$4\left(42.40^{\circ} \mathrm{API}\right)$} & \\
\hline & Trial 1 & Trial 2 & SD & Average & $\begin{array}{c}95 \% \\
\text { Confidence } \\
\text { interval }\end{array}$ & Trial 1 & Trial 2 & SD & Average & \\
\hline Volatile Hydrocarbons & 229.3 & 231.6 & 1.6 & 230.5 & $228.3-232.7$ & 225.5 & 223.1 & 1.7 & 224.3 & $221.9-226.7$ \\
\hline $\begin{array}{l}\text { Heteroaromatic } \\
\text { Compounds }\end{array}$ & 339.5 & 336.5 & 2.1 & 338.0 & $335.1-340.91$ & 332.6 & 330.7 & 1.3 & 331.7 & $329.9-333.5$ \\
\hline Polar Compounds & 216.2 & 216.8 & 0.4 & 216.5 & $215.9-217.1$ & 286.3 & 285.4 & 0.6 & 285.8 & $285.0-286.6$ \\
\hline $\begin{array}{l}\text { Heavy Saturated } \\
\text { Hydrocarbons }\end{array}$ & 269.8 & 278.5 & 6.2 & 274.2 & $265.6-282.8$ & 320.2 & 325.8 & 4.0 & 323.0 & $317.5-328.5$ \\
\hline $\begin{array}{l}\text { Alkyl Aromatic } \\
\text { Hydrocarbons }\end{array}$ & 410.8 & 422.0 & 7.9 & 416.4 & $405.5-427.4$ & 388.4 & 386.5 & 1.3 & 387.5 & $385.7-389.3$ \\
\hline $\begin{array}{l}\text { Overall Average Molecular } \\
\text { Weight of Condensate-like } \\
\text { Oil }\end{array}$ & 280.6 & 289.4 & 6.2 & 285.0 & 276.4-293.6 & 323.6 & 328.4 & 3.4 & 326.0 & $321.3-330.7$ \\
\hline
\end{tabular}

Table S11 continued

\begin{tabular}{|c|c|c|c|c|c|c|c|c|c|c|}
\hline \multirow[b]{3}{*}{ Chemical Classes } & \multicolumn{9}{|c|}{ Average molecular weight (Da) } & \\
\hline & \multicolumn{5}{|c|}{$3\left(41.62^{\circ} \mathrm{API}\right)$} & \multicolumn{5}{|c|}{$2\left(41.61^{\circ} \mathrm{API}\right)$} \\
\hline & Trial 1 & Trial 2 & SD & Average & $\begin{array}{c}95 \% \\
\text { Confidence } \\
\text { interval }\end{array}$ & Trial 1 & Trial 2 & SD & Average & $\begin{array}{c}95 \% \\
\text { Confidence } \\
\text { interval }\end{array}$ \\
\hline Volatile Hydrocarbons & 228.5 & 228.9 & 0.3 & 228.7 & $228.3-229.1$ & 225.2 & 227.9 & 1.9 & 226.6 & $224.0-229.2$ \\
\hline Heteroaromatic Compounds & 453.5 & 461.0 & 5.3 & 457.2 & $449.9-464.5$ & 475.5 & 472.0 & 2.5 & 473.8 & $470.3-477.3$ \\
\hline Polar Compounds & 240.1 & 236.8 & 2.3 & 238.4 & $235.2-241.6$ & 397.8 & 391.0 & 4.8 & 394.4 & $387.7-401.1$ \\
\hline $\begin{array}{l}\text { Heavy Saturated } \\
\text { Hydrocarbons }\end{array}$ & 312.6 & 313.1 & 0.3 & 312.9 & $312.5-313.3$ & 234.7 & 236.3 & 1.1 & 235.5 & $234.0-237.0$ \\
\hline $\begin{array}{l}\text { Alkyl Aromatic } \\
\text { Hydrocarbons }\end{array}$ & 379.1 & 363.0 & $\begin{array}{c}11 . \\
4\end{array}$ & 371.0 & $355.2-386.8$ & 387.4 & 383.0 & 3.1 & 385.2 & $380.9-389.5$ \\
\hline $\begin{array}{l}\text { Overall Average Molecular } \\
\text { Weight of the Condensate- } \\
\text { like Oil }\end{array}$ & 321.4 & 319.7 & 1.2 & 320.6 & $318.9-322.3$ & 253.5 & 254.3 & 0.6 & 253.9 & $253.1-254.7$ \\
\hline
\end{tabular}

Table S11 continued

$\frac{\text { Average molecular weight }(\mathrm{Da})}{1\left(37.50^{\circ} \mathrm{API}\right)}$

\begin{tabular}{|c|c|c|c|c|c|}
\hline \multirow[t]{2}{*}{ Chemical Classes } & & & & & $95 \%$ \\
\hline & Trial 1 & Trial 2 & SD & Average & $\begin{array}{c}\text { Confidence } \\
\text { interval }\end{array}$ \\
\hline Volatile Hydrocarbons & 231.3 & 234.2 & 2.1 & 232.8 & $229.9-235.7$ \\
\hline Heteroaromatic Compounds & 450.8 & 455.0 & 3.0 & 452.9 & $448.7-457.1$ \\
\hline Polar Compounds & 398.6 & 399.5 & 0.7 & 399.1 & $398.1-400.1$ \\
\hline Heavy Saturated Hydrocarbons & 307.3 & 308.0 & 0.5 & 307.6 & $306.9-308.3$ \\
\hline Alkyl Aromatic Hydrocarbons & 304.8 & 307.9 & 2.2 & 306.3 & 303.3-309.3 \\
\hline $\begin{array}{l}\text { Overall Average Molecular } \\
\text { Weight of the Condensate-like } \\
\text { Oil }\end{array}$ & 311.8 & 313.3 & 1.1 & 312.6 & $311.1-314.1$ \\
\hline
\end{tabular}




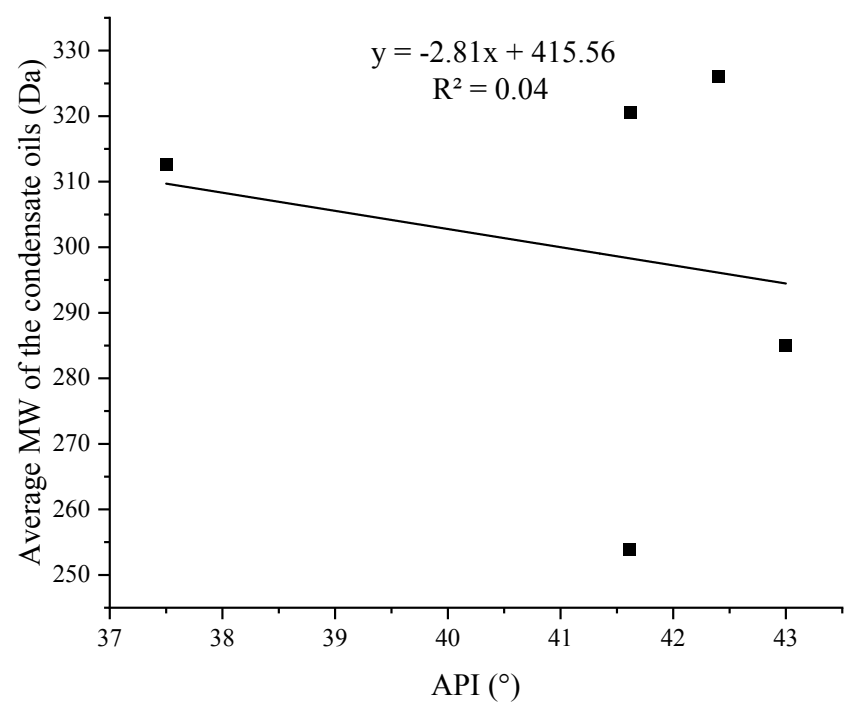

Figure S45. The overall average molecular weight of the condensate-like oil samples as a function of API gravity.

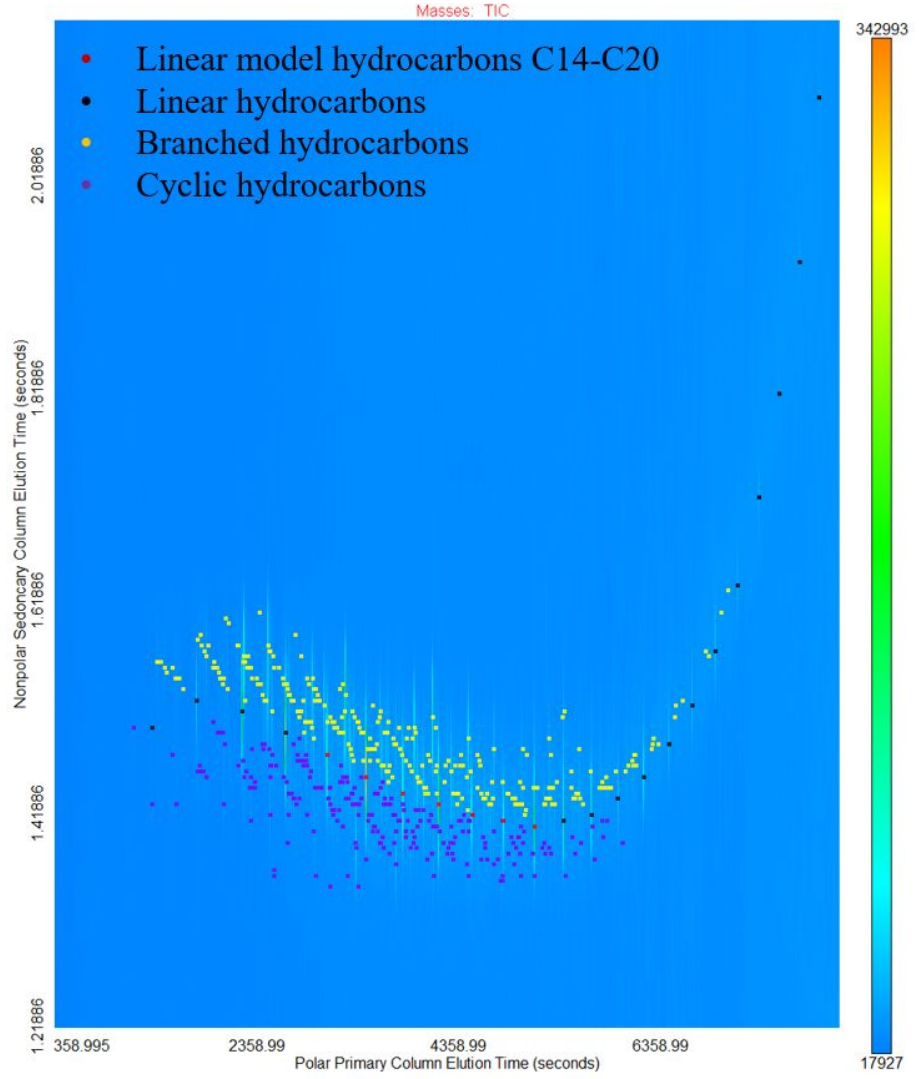

Figure S46. GC $\times \mathrm{GC} / \mathrm{TOF}$ chromatogram for the heavy saturated hydrocarbon fraction of oil 5 . 
Table S12. The RDBE values of compounds in the different compound classes of five different condensate-like oils. The average RDBE values of each individual compound class were determined based on the RDBE value of the ions and their abundances.

\begin{tabular}{|c|c|c|c|c|c|c|c|c|c|c|}
\hline & & \multirow{2}{*}{\multicolumn{9}{|c|}{ Average RDBE values }} \\
\hline & & & & & & & & & & \\
\hline & \multicolumn{5}{|c|}{$5\left(43.00^{\circ} \mathrm{API}\right)$} & \multicolumn{5}{|c|}{$4\left(42.40^{\circ} \mathrm{API}\right)$} \\
\hline Chemical Classes & Trial 1 & Trial 2 & S & Average & $\begin{array}{c}95 \% \\
\text { Confidence } \\
\text { interval }\end{array}$ & Trial 1 & Trial 2 & SD & Average & $\begin{array}{c}95 \% \\
\text { Confidence } \\
\text { interval }\end{array}$ \\
\hline Volatile Hydrocarbons & 1.9 & 1.5 & 0 & 1.7 & $1.3-2.1$ & 2.1 & 2.1 & - & 2.1 & - \\
\hline Heteroaromatic Compounds & 8.8 & 8.8 & 0 & 8.8 & - & 10.2 & 9.2 & 0.7 & 9.7 & $8.7-10.7$ \\
\hline Polar Compounds & 2.8 & 2.4 & 0 & 2.6 & $2.2-3.0$ & 6.0 & 5.9 & 0.1 & 5.9 & $5.8-6.0$ \\
\hline $\begin{array}{l}\text { Heavy Saturated } \\
\text { Hydrocarbons }\end{array}$ & 1.9 & 1.9 & 0 & 1.9 & - & 2.1 & 2.0 & 0.1 & 2.0 & $1.9-2.1$ \\
\hline $\begin{array}{l}\text { Alkyl Aromatic } \\
\text { Hydrocarbons }\end{array}$ & 7.2 & 7.4 & 0 . & 7.3 & $7.2-7.4$ & 6.4 & 5.4 & 0.7 & 5.9 & $6.9-4.9$ \\
\hline $\begin{array}{l}\text { Overall Average RDBE } \\
\text { Values of the Condensate- } \\
\text { like Oil }\end{array}$ & 2.4 & 2.4 & 0 & 2.4 & - & 2.5 & 2.4 & 0.1 & 2.4 & $2.3-2.5$ \\
\hline \multicolumn{11}{|l|}{ Table $\mathbf{S 1 2}$ continued } \\
\hline & \multicolumn{10}{|c|}{ Average RDBE values } \\
\hline & \multicolumn{5}{|c|}{$3\left(41.62^{\circ} \mathrm{API}\right)$} & \multicolumn{5}{|c|}{$2\left(41.61^{\circ} \mathrm{API}\right)$} \\
\hline Chemical Classes & Trial 1 & Trial 2 & $\mathrm{SD}$ & Average & $\begin{array}{l}5 \% \text { Confidence } \\
\text { interval }\end{array}$ & $\begin{array}{c}\text { Trial } \\
1\end{array}$ & Trial 2 & SD & Average & $\begin{array}{c}95 \% \\
\text { Confidence } \\
\text { interval }\end{array}$ \\
\hline Volatile Hydrocarbons & 2.5 & 2.5 & 0.1 & 2.5 & $2.4-2.6$ & 2.6 & 2.1 & 0.3 & 2.4 & $2.0-2.8$ \\
\hline Heteroaromatic Compounds & 9.0 & 9.2 & 0.1 & 9.1 & $9.0-9.2$ & 10.0 & 9.7 & 0.2 & 9.9 & $9.6-10.2$ \\
\hline Polar Compounds & 3.3 & 3.3 & 0.0 & 3.3 & - & 4.0 & 3.7 & 0.2 & 3.9 & $3.6-4.2$ \\
\hline $\begin{array}{l}\text { Heavy Saturated } \\
\text { Hydrocarbons }\end{array}$ & 2.8 & 2.8 & 0.0 & 2.8 & - & 1.6 & 1.6 & 0.0 & 1.6 & - \\
\hline $\begin{array}{l}\text { Alkyl Aromatic } \\
\text { Hydrocarbons }\end{array}$ & 5.0 & 5.3 & 0.2 & 5.2 & $4.9-5.5$ & 5.5 & 5.8 & 0.2 & 5.6 & $5.3-5.9$ \\
\hline $\begin{array}{l}\text { Overall Average RDBE } \\
\text { Values of the Condensate- } \\
\text { like Oil }\end{array}$ & 3.1 & 3.2 & 0.0 & 3.1 & - & 2.1 & 2.1 & 0.0 & 2.1 & - \\
\hline
\end{tabular}

Table S12 continued

\begin{tabular}{|c|c|c|c|c|c|}
\hline \multirow{4}{*}{ Chemical Classes } & \multicolumn{5}{|c|}{ Average RDBE values } \\
\hline & \multicolumn{5}{|c|}{$1\left(37.50^{\circ} \mathrm{API}\right)$} \\
\hline & & & & & $95 \%$ \\
\hline & Trial 1 & Trial 2 & $\mathrm{SD}$ & Average & $\begin{array}{l}\text { Confidence } \\
\text { interval }\end{array}$ \\
\hline Volatile Hydrocarbons & 3.3 & 2.5 & 0.6 & 2.9 & $2.1-3.7$ \\
\hline Heteroaromatic Compounds & 6.6 & 6.8 & 0.1 & 6.7 & $6.6-6.8$ \\
\hline Polar Compounds & 2.3 & 2.2 & 0.1 & 2.2 & $2.1-2.3$ \\
\hline Heavy Saturated Hydrocarbons & 2.8 & 2.8 & 0.0 & 2.8 & - \\
\hline Alkyl Aromatic Hydrocarbons & 4.6 & 4.6 & 0.1 & 4.6 & $4.5-4.7$ \\
\hline $\begin{array}{l}\text { Overall Average RDBE Values } \\
\text { of the Condensate-like Oil }\end{array}$ & 3.3 & 3.3 & 0.0 & 3.3 & - \\
\hline
\end{tabular}


A
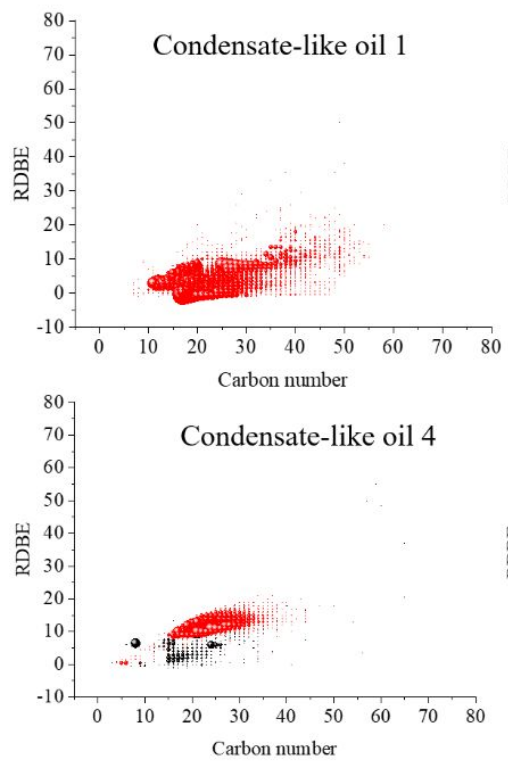

B
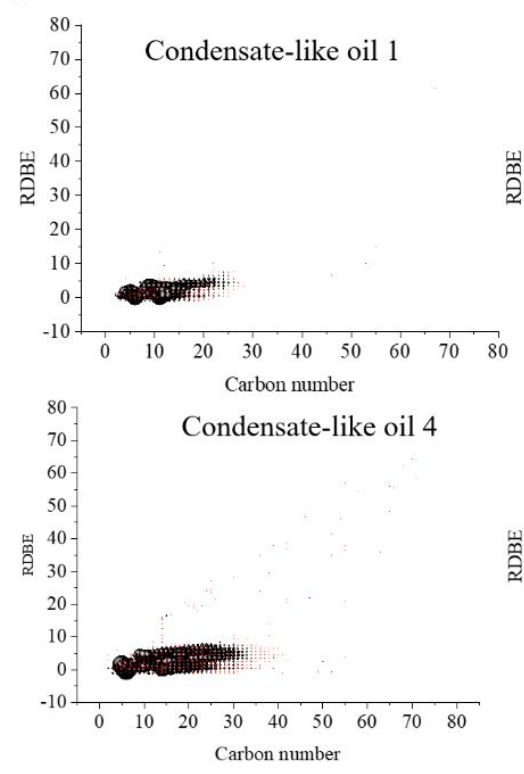

C
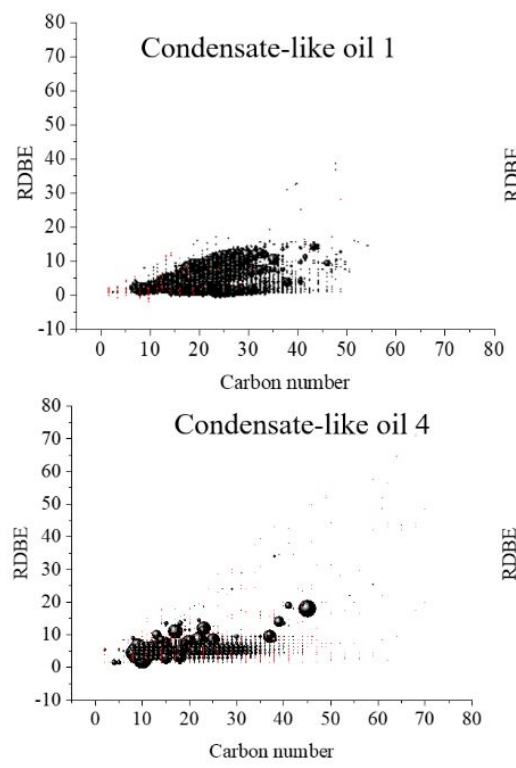
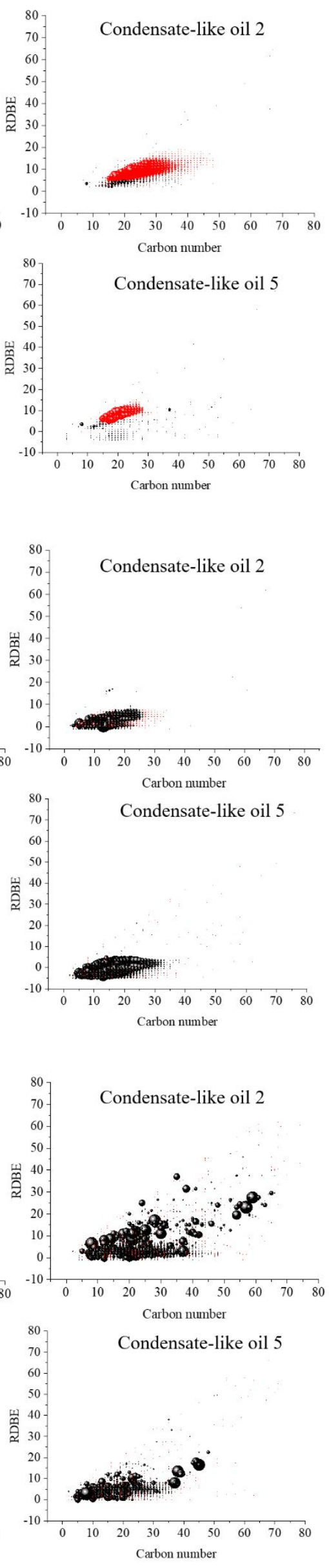
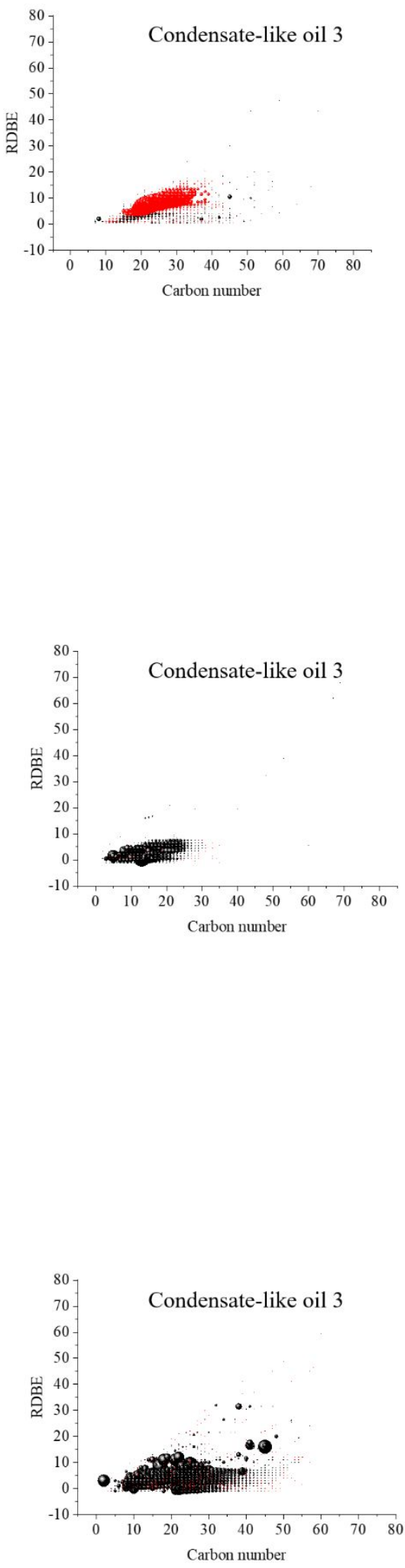
D
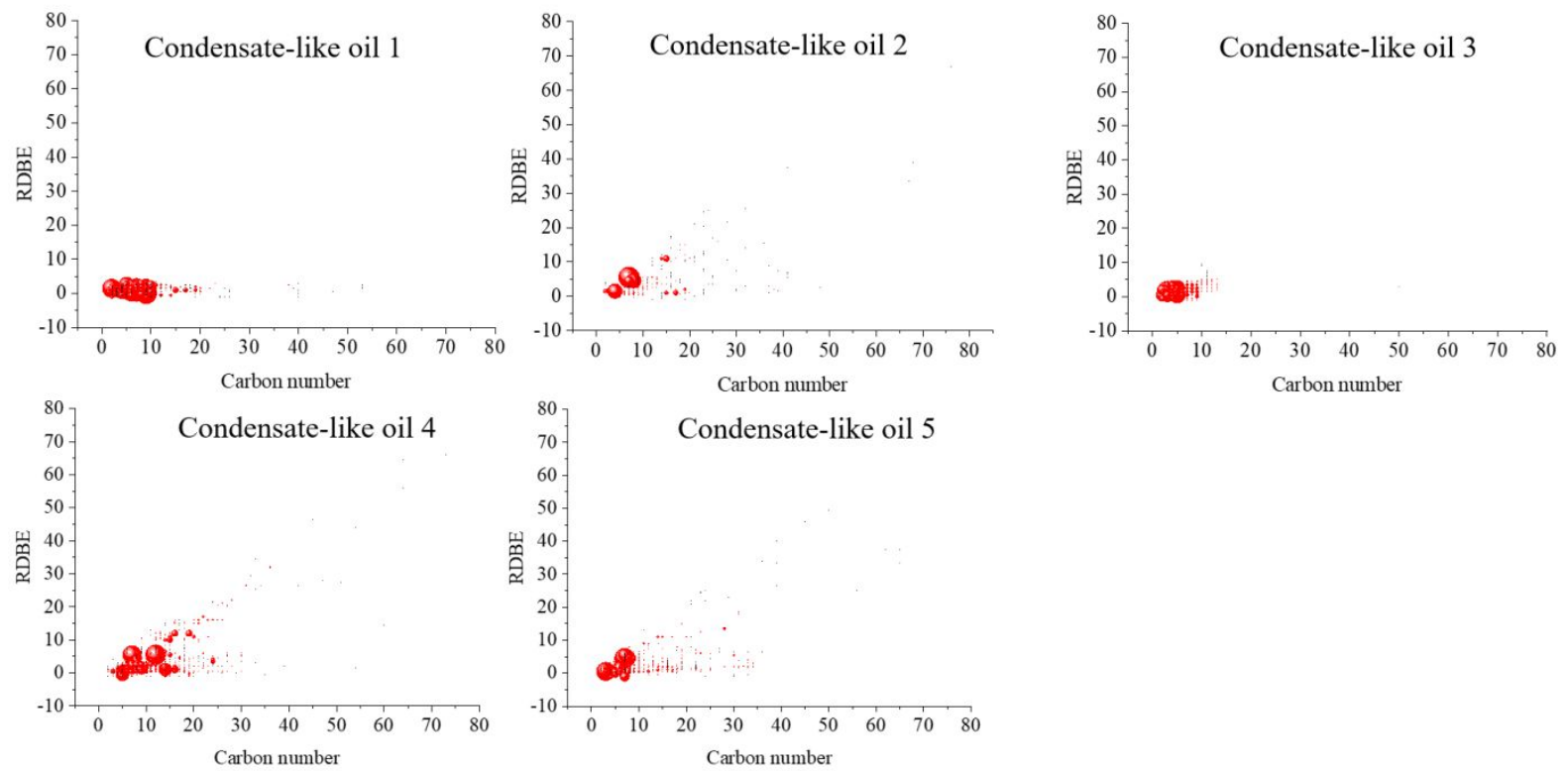

Figure S47. Color-mapped bubble plots of ring and double bond equivalent values (RDBE) versus carbon number for ionized compounds derived from the four nonvolatile condensate-like oil fractions that were separated and characterized by using the DPFMS method. A) Heteroaromatic compound fraction. B) Saturated and cyclic hydrocarbon fraction. C) Alkyl aromatic hydrocarbon fraction. D) Polar compound fraction. Bubble size indicates the relative abundance of the ions. The black bubbles represent ions containing only $\mathrm{C}$ and $\mathrm{H}$. Red bubbles represent ions containing heteroatoms (N, O and/or S) along with $\mathrm{C}$ and $\mathrm{H}$. 
A
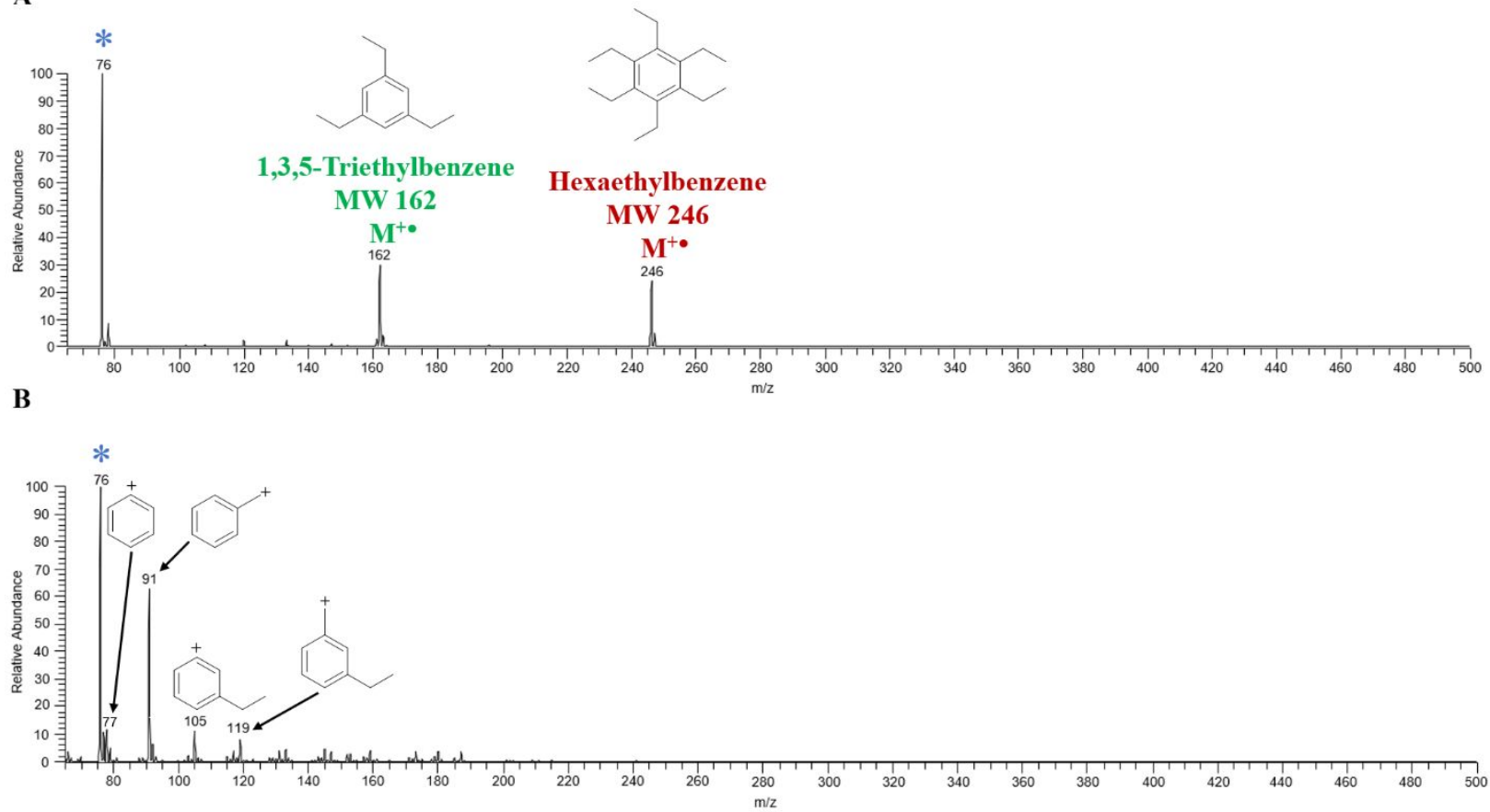

C

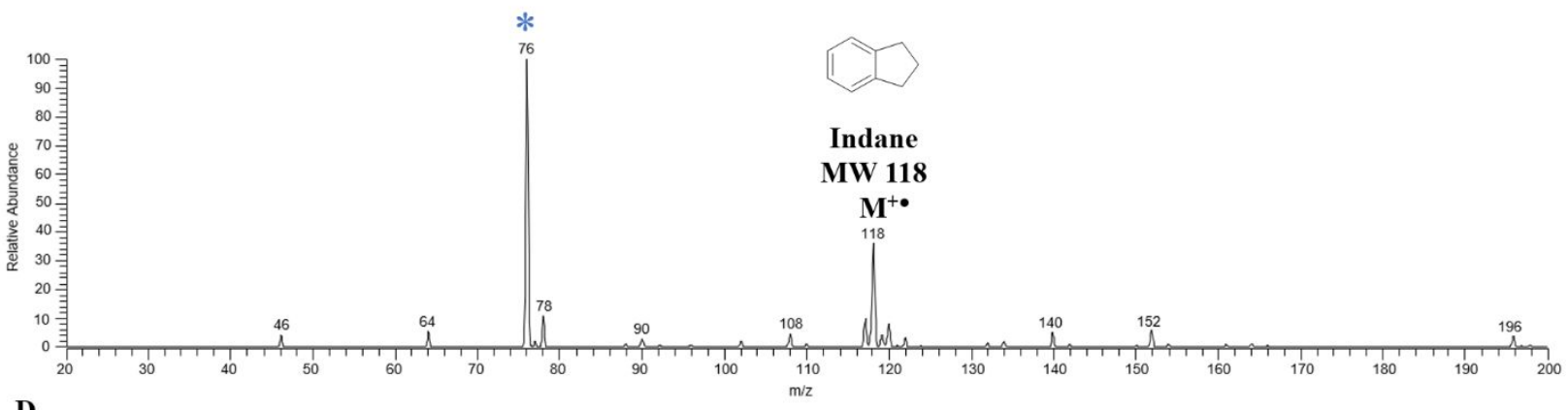

D

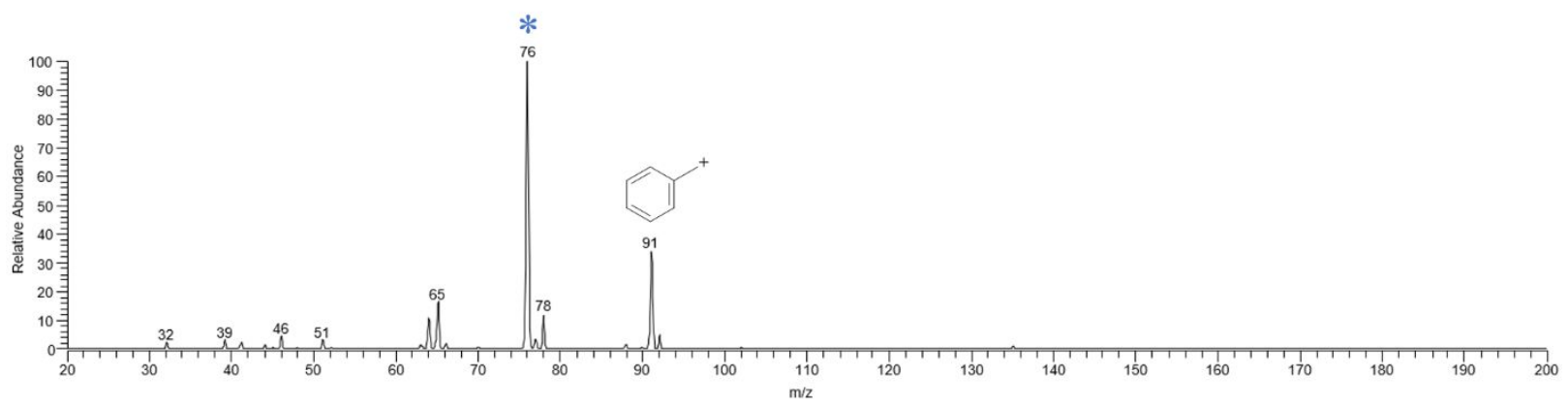

Figure S48. A) (+) APCI/ $\mathrm{N}_{2} / \mathrm{CS}_{2}$ low resolution mass spectrum of an equimolar $(1 \mathrm{mM})$ mixture of two alkylaromatic hydrocarbon model compounds. B) ISCAD mass spectrum of the mixture of the two ionized alkylaromatic model compounds, illustrating the ability to use ISCAD to cleave off most of the alkyl substituents on the aromatic cores. C) (+) APCI/ $\mathrm{N}_{2} / \mathrm{CS}_{2}$ low resolution mass spectrum of $1 \mathrm{mM}$ solution of indane. D) ISCAD mass spectrum of the ions shown in part $\mathrm{C}$, including indane molecular ions. Peaks at $m / z 76$ designated with a blue asterisk $(*)$ are due to the molecular ion of $\mathrm{CS}_{2}$. 


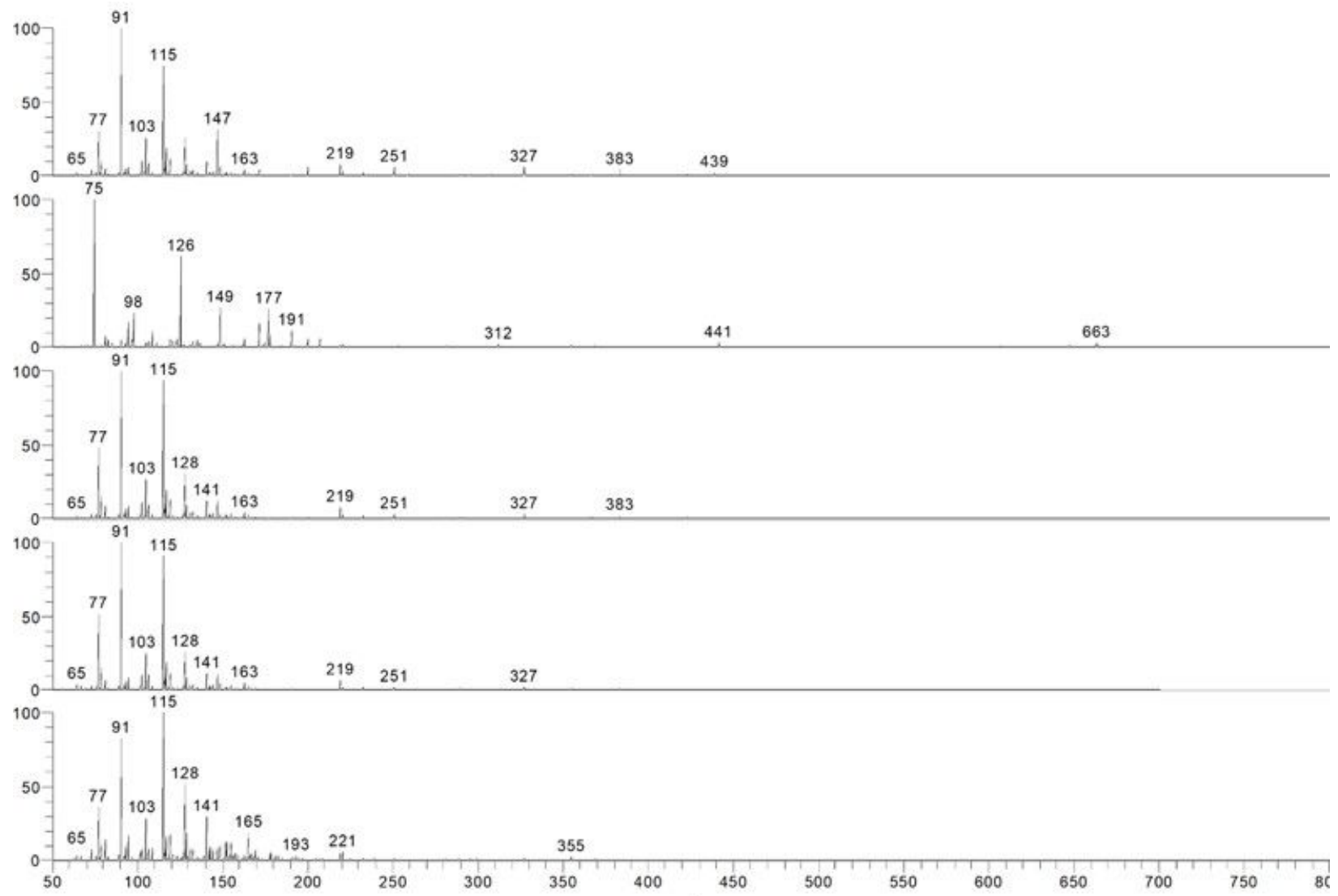

Figure 3S49. (+) APCI/CS $\mathrm{CS}_{2}$ mass spectra after ISCAD for the ionized compounds in the alkyl aromatic hydrocarbon classes of the five condensate-like oils from top to bottom in this order: 4, 5, 3, 2, and 1.

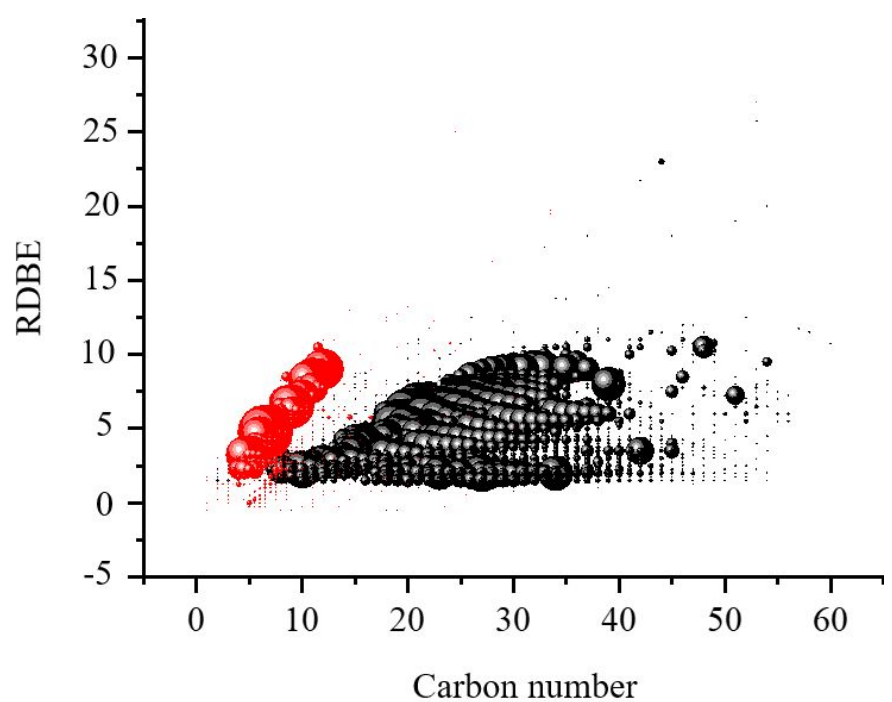

Figure S504. Ring and double bond equivalent (RDBE) versus carbon number plot for APCI mass spectrum (black) and ISCAD spectrum (red) of the alkyl aromatic hydrocarbon fraction of condensate-like oil 1. 
Table 1S13. The MW of the aromatic cores and the total number of carbons in the alkyl side chains of the alkyl aromatic hydrocarbons with averages and standard deviations from two experiments for the five condensate-like oils. API gravities are shown in parenthesis

\begin{tabular}{|c|c|c|c|c|c|c|c|c|c|c|}
\hline & & & \multicolumn{8}{|c|}{$\begin{array}{c}\text { The average MW of the aromatic cores and the average total number of carbons in the } \\
\text { alkyl side chains of the alkyl aromatic hydrocarbons }\end{array}$} \\
\hline & \multicolumn{5}{|c|}{$5\left(43.00^{\circ} \mathrm{API}\right)$} & \multicolumn{5}{|c|}{$4\left(42.40^{\circ} \mathrm{API}\right)$} \\
\hline & Trial 1 & Trial 2 & $\mathrm{SD}$ & Average & $\begin{array}{c}95 \% \\
\text { Confidence } \\
\text { interval }\end{array}$ & Trial 1 & Trial 2 & SD & Average & $\begin{array}{c}95 \% \\
\text { Confidence } \\
\text { interval }\end{array}$ \\
\hline $\begin{array}{l}\text { MW of the Aromatic Cores } \\
\text { (Da) }\end{array}$ & 125.9 & 125.8 & 0.1 & 125.8 & $125.7-125.9$ & 127.1 & 127.6 & 0.4 & 127.4 & $126.8-128.0$ \\
\hline $\begin{array}{l}\text { Total Number of Carbons in } \\
\text { the Alkyl Side Chains }\end{array}$ & 22.2 & 21.4 & 0.6 & 21.8 & $21.0-22.6$ & 19.7 & 19.5 & 0.1 & 19.6 & $19.5-19.7$ \\
\hline
\end{tabular}

Table $\mathbf{S 1 3}$ continued

\begin{tabular}{|c|c|c|c|c|c|c|c|c|c|c|}
\hline & \multicolumn{10}{|c|}{$\begin{array}{l}\text { The average MW of the aromatic cores and the average total number of carbons in the alkyl side chains of } \\
\text { the alkyl aromatic hydrocarbons }\end{array}$} \\
\hline & \multicolumn{5}{|c|}{$3\left(41.62^{\circ} \mathrm{API}\right)$} & \multicolumn{5}{|c|}{$2\left(41.61^{\circ} \mathrm{API}\right)$} \\
\hline & Trial 1 & Trial 2 & SD & Average & $\begin{array}{c}95 \% \\
\text { Confidence } \\
\text { interval }\end{array}$ & $\begin{array}{c}\text { Trial } \\
1\end{array}$ & Trial 2 & SD & Average & $\begin{array}{c}95 \% \\
\text { Confidence } \\
\text { interval }\end{array}$ \\
\hline $\begin{array}{l}\text { MW of the Aromatic Cores } \\
\text { (Da) }\end{array}$ & 126.1 & 126.2 & 0.0 & 126.2 & - & $\begin{array}{c}112 . \\
6\end{array}$ & 113.2 & 0.4 & 112.9 & $112.3-113.5$ \\
\hline $\begin{array}{l}\text { Total Number of Carbons in } \\
\text { the Alkyl Side Chains }\end{array}$ & 19.1 & 17.9 & 0.8 & 18.5 & $17.4-19.6$ & 20.6 & 20.3 & 0.3 & 20.4 & $20.0-20.8$ \\
\hline
\end{tabular}

Table S13 continued

\begin{tabular}{|c|c|c|c|c|c|}
\hline & & \multirow{2}{*}{\multicolumn{4}{|c|}{$\begin{array}{l}\text { The average MW of the aromatic cores and the } \\
\text { average total number of carbons in the alkyl side } \\
\text { chains of the alkyl aromatic hydrocarbons } \\
1\left(37.50^{\circ} \mathrm{API}\right)\end{array}$}} \\
\hline & & & & & \\
\hline & Trial 1 & Trial 2 & SD & Average & $\begin{array}{c}95 \% \\
\text { Confidence } \\
\text { interval }\end{array}$ \\
\hline $\begin{array}{l}\text { MW of the Aromatic Cores } \\
\text { (Da) }\end{array}$ & 123.8 & 124.4 & 0.4 & 124.1 & $123.5-124.7$ \\
\hline $\begin{array}{l}\text { Total Number of Carbons in } \\
\text { the Alkyl Side Chains }\end{array}$ & 13.9 & 14.1 & 0.1 & 14.0 & $13.9-14.1$ \\
\hline
\end{tabular}

Table S14. Data obtained for compounds in the distillate fraction of condensate-like oil 2 by using GCxGC/(+EI) TOF MS. Data provided are based on ChromaTOF software prediction of the molecular ion $\mathrm{m} / \mathrm{z}$ value and the identification of the compound by using the EI mass spectral library match using a match factor threshold of 800. Formulas with a question mark "?" might not have been accurately identified. The ratio of the peak area of each of these questionable compounds relative to all peak areas of the detected compounds is provided in the table below.

\begin{tabular}{|l|c|c|c|c|c|}
\hline \multicolumn{1}{|c|}{ Name } & Formula & $\begin{array}{c}\text { Retention Times (s) on the } \\
\text { Primary and Secondary } \\
\text { Columns }\end{array}$ & $\begin{array}{c}\text { Molecular } \\
\text { Ion m/z }\end{array}$ & $\begin{array}{c}\text { Chromatographic } \\
\text { Peak Area }\end{array}$ & $\begin{array}{c}\text { Ratio } \\
\text { (Peak area/sum of all } \\
\text { peak areas) }\end{array}$ \\
\hline 3-Methylheptane & $\mathrm{C}_{8} \mathrm{H}_{18}$ & $406.002,2.412$ & 114.1403 & 403434 \\
\hline 4-Methylpropiolactam & $\mathrm{C}_{4} \mathrm{H}_{7} \mathrm{NO}$ & $418.005,2.389$ & 85.0522 & 121454 \\
\hline 3,3-Dipropylcyclobutanone & $\mathrm{C}_{10} \mathrm{H}_{18} \mathrm{O} ?$ & $424.007,2.314$ & 154.1352 & 14514 \\
\hline 4-Octen-3-one & $\mathrm{C}_{8} \mathrm{H}_{14} \mathrm{O}$ & $430.009,2.389$ & 126.1039 & 1293932 \\
\hline Octane & $\mathrm{C}_{8} \mathrm{H}_{18}$ & $442.012,2.607$ & 114.1403 & 112.1247 & 4353762 \\
\hline cis-1,3-Dimethylcyclohexane & $\mathrm{C}_{8} \mathrm{H}_{16}$ & $454.016,2.391$ & & 2 \\
\hline
\end{tabular}




\begin{tabular}{|c|c|c|c|c|c|}
\hline $\begin{array}{l}2,2,3,3- \\
\text { Tetramethylcyclobutanone }\end{array}$ & $\mathrm{C}_{8} \mathrm{H}_{14} \mathrm{O}$ & $454.016,2.616$ & 126.1039 & 27788 & \\
\hline 2,2,4-Trimethylhexane & $\mathrm{C}_{9} \mathrm{H}_{20}$ & $460.017,2.798$ & 128.156 & 435562 & \\
\hline 2,4-Dimethylheptane & $\mathrm{C}_{9} \mathrm{H}_{20}$ & $466.019,2.823$ & 128.156 & 176761 & \\
\hline 1,1-Dimethylcyclohexane & $\mathrm{C}_{8} \mathrm{H}_{16}$ & $478.022,2.427$ & 112.1247 & 705398 & \\
\hline 2,6-Dimethylheptane & $\mathrm{C}_{9} \mathrm{H}_{20}$ & $478.022,2.885$ & 128.156 & 574057 & \\
\hline 2-Octyl ethynyl ether & $\mathrm{C}_{10} \mathrm{H}_{18} \mathrm{O}$ & $484.024,2.520$ & 154.1352 & 76398 & \\
\hline 1,2-Dimethylcyclohexane & $\mathrm{C}_{8} \mathrm{H}_{16}$ & $496.028,2.510$ & 112.1247 & 1051584 & \\
\hline 3,5-Dimethylheptane & $\mathrm{C}_{9} \mathrm{H}_{20}$ & $496.028,2.931$ & 128.156 & 2397681 & \\
\hline 1,4-Dimethylcyclohexane & $\mathrm{C}_{8} \mathrm{H}_{16}$ & $514.033,2.546$ & 112.1247 & 1076015 & \\
\hline 1,3,5-Triamine pentane & $\mathrm{C}_{5} \mathrm{H}_{15} \mathrm{~N}_{3}$ & $526.036,2.844$ & 117.126 & 3200 & \\
\hline 3-Ethyl-2,4-dimethylpentane & $\mathrm{C}_{9} \mathrm{H}_{20}$ & $526.036,3.007$ & 128.156 & 52768 & \\
\hline $\begin{array}{l}\text { 1,3,5-Trimethyl-, } \\
\text { (1à,3à,5à)cyclohexane }\end{array}$ & $\mathrm{C}_{9} \mathrm{H}_{18}$ & $538.04,2.782$ & 126.1403 & 750720 & \\
\hline Tuberolactone & $\mathrm{C}_{10} \mathrm{H}_{14} \mathrm{O}_{2}$ & $538.04,2.903$ & 166.0988 & 8658 & \\
\hline 1,1,3-Trimethylcyclohexane & $\mathrm{C}_{9} \mathrm{H}_{18}$ & $556.045,2.829$ & 126.1403 & 1489200 & \\
\hline $\begin{array}{l}\text { 2,3,3,6,6,7-Hexamethyl-1,4,7- } \\
\text { octatriene }\end{array}$ & $\mathrm{C}_{14} \mathrm{H}_{24}$ & $556.045,2.875$ & 192.1873 & 38408 & \\
\hline 1-Bicyclo[2.2.2]octanol & $\mathrm{C}_{8} \mathrm{H}_{14} \mathrm{O}$ ? & $574.05,2.925$ & 126.1039 & 7147 & $6.40 \mathrm{E}-5$ \\
\hline 3-Methyloctane & $\mathrm{C}_{9} \mathrm{H}_{20}$ & $580.052,3.209$ & 128.156 & 3164154 & \\
\hline Ethylcyclohexane & $\mathrm{C}_{8} \mathrm{H}_{16}$ & $586.053,2.700$ & 112.1247 & 1128224 & \\
\hline 1,2,4-Trimethyl-cyclohexane & $\mathrm{C}_{9} \mathrm{H}_{18}$ & $592.055,2.921$ & 126.1403 & 1193093 & \\
\hline 2,2,5-trimethylhexane & $\mathrm{C}_{9} \mathrm{H}_{20}$ & $592.055,3.403$ & 128.156 & 20793 & \\
\hline Toluene & $\mathrm{C}_{7} \mathrm{H}_{8}$ & $598.057,2.067$ & 92.0621 & 209836 & \\
\hline 2-Oxepanone & $\mathrm{C}_{6} \mathrm{H}_{10} \mathrm{O}_{2}$ & $616.062,3.007$ & 114.0675 & 9329 & \\
\hline $\begin{array}{l}\text { (Z)-3-(Hydroxymethyl)-7- } \\
\text { methylocta-2,6-dien-1-yl } \\
\text { acetate }\end{array}$ & $\mathrm{C}_{12} \mathrm{H}_{20} \mathrm{O}_{3}$ ? & $616.062,3.025$ & 212.1407 & 9522 & $8.60 \mathrm{E}-5$ \\
\hline 3,3-Dimethylheptane & $\mathrm{C}_{9} \mathrm{H}_{20}$ & $628.065,3.517$ & 128.156 & 73612 & \\
\hline $\begin{array}{l}\text { O-(tert-Butoxycarbonyl)-N- } \\
\text { isopropylidene oxime }\end{array}$ & $\mathrm{C}_{8} \mathrm{H}_{15} \mathrm{NO}_{3}$ & $646.071,3.447$ & 173.1046 & 13770 & \\
\hline $\begin{array}{l}1,1,3,5- \\
\text { Tetramethylcyclohexane }\end{array}$ & $\mathrm{C}_{10} \mathrm{H}_{20}$ & $652.072,3.222$ & 140.156 & 197139 & \\
\hline Nonane & $\mathrm{C}_{9} \mathrm{H}_{20}$ & $658.074,3.405$ & 128.156 & 2970311 & \\
\hline $\begin{array}{l}\text { 1,2,3-Trimethyl-, (1à,2à,3á)- } \\
\text { cyclohexane }\end{array}$ & $\mathrm{C}_{9} \mathrm{H}_{18}$ & $670.078,3.055$ & 126.1403 & 469884 & \\
\hline Methylcycloheptane & $\mathrm{C}_{8} \mathrm{H}_{16}$ & $676.079,2.781$ & 112.1247 & 8641 & \\
\hline
\end{tabular}




\begin{tabular}{|c|c|c|c|c|}
\hline 2,4-Dimethylhexane & $\mathrm{C}_{8} \mathrm{H}_{18}$ & $676.079,3.667$ & 114.1403 & 912814 \\
\hline 3,5-Dimethyloctane & $\mathrm{C}_{10} \mathrm{H}_{22}$ & $700.086,3.718$ & 142.1716 & 1901320 \\
\hline 1-Ethyl-4-methylcyclohexane & $\mathrm{C}_{9} \mathrm{H}_{18}$ & $706.088,3.101$ & 126.1403 & 2103969 \\
\hline $\begin{array}{l}1,1,3,5- \\
\text { Tetramethylcyclohexane }\end{array}$ & $\mathrm{C}_{10} \mathrm{H}_{20}$ & $718.091,3.338$ & 140.156 & 273592 \\
\hline 2,6-Dimethyloctane & $\mathrm{C}_{10} \mathrm{H}_{22}$ & $730.095,3.770$ & 142.1716 & 1638518 \\
\hline (E)-1,3-Hexadiene & $\mathrm{C}_{6} \mathrm{H}_{10}$ & $748.1,2.760$ & 82.0777 & 86271 \\
\hline 3-Methylnonane & $\mathrm{C}_{10} \mathrm{H}_{22}$ & $760.103,3.785$ & 142.1716 & 825709 \\
\hline 1-Ethyl-2-methylcyclohexane & $\mathrm{C}_{9} \mathrm{H}_{18}$ & $778.109,3.178$ & 126.1403 & 941838 \\
\hline Heptanal & $\mathrm{C}_{7} \mathrm{H}_{14} \mathrm{O}$ & $790.112,3.792$ & 114.1039 & 109601 \\
\hline 3,3,6-Trimethyl-1,2-dioxane & $\mathrm{C}_{7} \mathrm{H}_{13} \mathrm{O}_{2}$ & $802.115,3.608$ & 129.091 & 8287 \\
\hline 1-Ethyl-1-methylcyclohexane & $\mathrm{C}_{9} \mathrm{H}_{18}$ & $808.117,3.153$ & 126.1403 & 193199 \\
\hline 5-Methylundecane & $\mathrm{C}_{12} \mathrm{H}_{26}$ & $814.119,3.862$ & 170.2029 & 1393101 \\
\hline (1-Methylethyl)cyclohexane & $\mathrm{C}_{9} \mathrm{H}_{18}$ & $832.124,3.204$ & 126.1403 & 168744 \\
\hline 4-Methylnonane & $\mathrm{C}_{10} \mathrm{H}_{22}$ & $832.124,3.929$ & 142.1716 & 2992154 \\
\hline 3-Nonyne & $\mathrm{C}_{9} \mathrm{H}_{16}$ & $838.126,2.978$ & 124.1247 & 147187 \\
\hline $\begin{array}{l}\text { 1-Ethyl-2,4- } \\
\text { dimethylcyclohexane }\end{array}$ & $\mathrm{C}_{10} \mathrm{H}_{20}$ & $838.126,3.502$ & 140.156 & 555003 \\
\hline $\begin{array}{l}\text { (R)-3-(Tert- } \\
\text { butylsulfinyl)butanoic acid }\end{array}$ & $\mathrm{C}_{8} \mathrm{H}_{16} \mathrm{O}_{3} \mathrm{~S}$ & $838.126,4.209$ & 192.0815 & 129278 \\
\hline Decane & $\mathrm{C}_{10} \mathrm{H}_{22}$ & $844.127,4.173$ & 142.1716 & 79269 \\
\hline 3-Methyldodecane & $\mathrm{C}_{13} \mathrm{H}_{28}$ & $850.129,4.225$ & 184.2186 & 57459 \\
\hline Propylcyclohexane & $\mathrm{C}_{9} \mathrm{H}_{18}$ & $856.131,3.292$ & 126.1403 & 759545 \\
\hline Octahydro-cis- $1 \mathrm{H}$-indene & $\mathrm{C}_{9} \mathrm{H}_{16}$ & $874.136,3.106$ & 124.1247 & 144668 \\
\hline $\begin{array}{l}\text { 1-Ethyl-4-methyl-trans- } \\
\text { cyclohexane }\end{array}$ & $\mathrm{C}_{9} \mathrm{H}_{18}$ & $874.136,3.225$ & 126.1403 & 100699 \\
\hline 1-Ethyl-2-propylcyclohexane & $\mathrm{C}_{11} \mathrm{H}_{22}$ & $874.136,3.533$ & 154.1716 & 203834 \\
\hline $\begin{array}{l}\text { 1-Methyl-2-methylene-3-(1- } \\
\text { methylethyl)-cis- cyclopentane }\end{array}$ & $\mathrm{C}_{10} \mathrm{H}_{18}$ & $880.138,3.375$ & 138.1403 & 96215 \\
\hline Ethylbenzene & $\mathrm{C}_{8} \mathrm{H}_{10}$ & $886.14,2.378$ & 106.0777 & 68545 \\
\hline Cyclobutylidenacetaldehyde & $\mathrm{C}_{6} \mathrm{H}_{8} \mathrm{O}$ & $898.143,3.383$ & 96.057 & 32889 \\
\hline $\begin{array}{l}\text { 1-Ethyl-1,3-dimethyl-trans- } \\
\text { cyclohexane }\end{array}$ & $\mathrm{C}_{10} \mathrm{H}_{20}$ & $898.143,3.516$ & 140.156 & 168787 \\
\hline 1,3-Dimethylbenzene, & $\mathrm{C}_{8} \mathrm{H}_{10}$ & $904.145,2.417$ & 106.0777 & 820707 \\
\hline 4-Methylundecane & $\mathrm{C}_{12} \mathrm{H}_{26}$ & $916.148,4.251$ & 170.2029 & 77287 \\
\hline
\end{tabular}




\begin{tabular}{|c|c|c|c|c|c|}
\hline 3,3-Dimethyl-2-oxetanone & $\mathrm{C}_{5} \mathrm{H}_{8} \mathrm{O}_{2}$ & $946.157,3.456$ & 100.0519 & 10373 & \\
\hline $\begin{array}{l}\text { Dimethyl ester-2-butynedioic } \\
\text { acid }\end{array}$ & $\mathrm{C}_{6} \mathrm{H}_{6} \mathrm{O}_{4}$ & $946.157,3.543$ & 142.0261 & 27280 & \\
\hline Undecane & $\mathrm{C}_{11} \mathrm{H}_{24}$ & $952.158,4.297$ & 156.1873 & 188480 & \\
\hline $\begin{array}{l}\text { 1-(2-Furanyl)-4-methyl-1- } \\
\text { penten-3-ol }\end{array}$ & $\mathrm{C}_{10} \mathrm{H}_{14} \mathrm{O}_{2} ?$ & $964.162,3.579$ & 166.0988 & 26015 & 2.34E-4 \\
\hline $\begin{array}{l}\text { Ethyl 2-cyanohexa-4,5- } \\
\text { dienoate }\end{array}$ & $\mathrm{C}_{9} \mathrm{H}_{11} \mathrm{NO}_{2} ?$ & $970.164,4.140$ & 165.0784 & 14041 & $1.26 \mathrm{E}-4$ \\
\hline $\begin{array}{l}\text { 1-Isopropyl-4- } \\
\text { methylcyclohexane }\end{array}$ & $\mathrm{C}_{10} \mathrm{H}_{20}$ & $982.167,3.595$ & 140.156 & 414672 & \\
\hline Dodecane & $\mathrm{C}_{12} \mathrm{H}_{26}$ & $988.169,4.341$ & 170.2029 & 650880 & \\
\hline $\begin{array}{l}\text { 1-Butyl-2- } \\
\text { methylenecyclopentane }\end{array}$ & $\mathrm{C}_{10} \mathrm{H}_{18}$ & $994.171,3.154$ & 138.1403 & 29059 & \\
\hline 1-Hexyne & $\mathrm{C}_{6} \mathrm{H}_{10}$ & $1012.18,3.472$ & 82.0777 & 31352 & \\
\hline 1-Ethylcyclohexene & $\mathrm{C}_{8} \mathrm{H}_{14}$ & $1018.18,3.456$ & 110.109 & 106299 & \\
\hline $\begin{array}{l}\text { 1-Methyl-3-(1-methylethyl)- } \\
\text { cyclohexane }\end{array}$ & $\mathrm{C}_{10} \mathrm{H}_{20}$ & $1018.18,3.672$ & 140.156 & 506003 & \\
\hline 2,6-Dimethylnonane & $\mathrm{C}_{11} \mathrm{H}_{24}$ & $1024.18,4.356$ & 156.1873 & 1352944 & \\
\hline (E)-4-Hexen-1-ol & $\mathrm{C}_{6} \mathrm{H}_{12} \mathrm{O}$ & $1048.19,3.093$ & 100.0883 & 10630 & \\
\hline Undec-3-en-5-yn-2-ol & $\mathrm{C}_{11} \mathrm{H}_{18} \mathrm{O}$ ? & $1048.19,3.335$ & 166.1352 & 39074 & $3.52 \mathrm{E}-5$ \\
\hline Pinane-10-diol & $\mathrm{C}_{10} \mathrm{H}_{18} \mathrm{O}_{2}$ & $1054.19,3.911$ & 170.1301 & 29783 & \\
\hline 6-Ethyl-2-methyloctane & $\mathrm{C}_{11} \mathrm{H}_{24}$ & $1054.19,4.335$ & 156.1873 & 645744 & \\
\hline $\begin{array}{l}\text { 2-Ethylcyclohexyl 2- } \\
\text { thiopheneacetate }\end{array}$ & $\mathrm{C}_{14} \mathrm{H}_{20} \mathrm{O}_{2} \mathrm{~S}$ ? & $1072.19,3.646$ & 252.1179 & 394899 & $3.55 \mathrm{E}-3$ \\
\hline 2,6,11-Trimethyldodecane & $\mathrm{C}_{15} \mathrm{H}_{32}$ & $1078.19,4.371$ & 212.2499 & 613681 & \\
\hline $\begin{array}{l}\text { 1-Ethyl-4-methyl-cis- } \\
\text { cyclohexane }\end{array}$ & $\mathrm{C}_{9} \mathrm{H}_{18}$ & $1096.2,3.636$ & 126.1403 & 266918 & \\
\hline 4-Phenylpent-1-ene & $\mathrm{C}_{11} \mathrm{H}_{14}$ & $1102.2,2.577$ & 146.109 & 23369 & \\
\hline 3,7-Dimethylnonane & $\mathrm{C}_{11} \mathrm{H}_{24}$ & $1102.2,4.356$ & 156.1873 & 619490 & \\
\hline $\begin{array}{l}\text { Methyl octadeca-13,14- } \\
\text { dienoate }\end{array}$ & $\mathrm{C}_{19} \mathrm{H}_{34} \mathrm{O}_{2} ?$ & $1114.2,3.481$ & 294.2553 & 16052 & $1.44 \mathrm{E}-4$ \\
\hline 3-Ethoxy-1-propyne & $\mathrm{C}_{5} \mathrm{H}_{8} \mathrm{O}$ & $1126.21,3.925$ & 84.057 & 53546 & \\
\hline $\begin{array}{l}\text { 1-(Dodecycloxy)-4- } \\
\text { methoxybutan-2-one }\end{array}$ & $\mathrm{C}_{17} \mathrm{H}_{34} \mathrm{O}_{3} ?$ & $1126.21,4.314$ & 286.2502 & 20112 & $1.81 \mathrm{E}-4$ \\
\hline 1-Cyclooctyl-1-propanone & $\mathrm{C}_{11} \mathrm{H}_{20} \mathrm{O}$ & $1132.21,3.949$ & 168.1509 & 184729 & \\
\hline 2-Methylundecane & $\mathrm{C}_{12} \mathrm{H}_{26}$ & $1132.21,4.712$ & 170.2029 & 44258 & \\
\hline Pentadecane & $\mathrm{C}_{15} \mathrm{H}_{32}$ & $1150.22,4.407$ & 212.2499 & 506831 & \\
\hline (E)-Dec-5-en-2-ol & $\mathrm{C}_{10} \mathrm{H}_{20} \mathrm{O} ?$ & $1168.22,3.773$ & 156.1509 & 19617 & $1.76 \mathrm{E}-4$ \\
\hline 5-Methyldecane & $\mathrm{C}_{11} \mathrm{H}_{24}$ & $1174.22,4.423$ & 156.1873 & 1473756 & \\
\hline
\end{tabular}




\begin{tabular}{|c|c|c|c|c|c|}
\hline $\begin{array}{l}\text { 3,6-Dimethyl-2- } \\
\text { benzo[b]furanone }\end{array}$ & $\mathrm{C}_{10} \mathrm{H}_{16} \mathrm{O}_{2}$ & $1180.22,3.354$ & 168.1145 & 21300 & \\
\hline Dibutyl ethanedioate & $\mathrm{C}_{10} \mathrm{H}_{18} \mathrm{O}_{4}$ & $1180.22,4.728$ & 202.12 & 33721 & \\
\hline $\begin{array}{l}\text { 5-Cyclohexylidene--1-penten- } \\
\text { 3-ol }\end{array}$ & $\mathrm{C}_{11} \mathrm{H}_{18} \mathrm{O} ?$ & $1186.23,3.810$ & 166.1352 & 9778 & $8.80 \mathrm{E}-5$ \\
\hline $\begin{array}{l}\text { 2-(Aminomethyl)-cis- } \\
\text { cyclopropanecarboxylic acid }\end{array}$ & $\mathrm{C}_{5} \mathrm{H}_{9} \mathrm{NO}_{2}$ & $1186.23,3.985$ & 115.0628 & 18101 & \\
\hline 4-Methyldecane & $\mathrm{C}_{11} \mathrm{H}_{24}$ & $1186.23,4.433$ & 156.1873 & 451862 & \\
\hline $\begin{array}{l}\text { 4',4'-Dimethylspiro[1,3- } \\
\text { dioxolane-2,3'-cholestan-7'- } \\
\text { one] }\end{array}$ & $\mathrm{C}_{31} \mathrm{H}_{52} \mathrm{O}_{3} ?$ & $1192.23,4.504$ & 472.3911 & 4604 & $4.14 \mathrm{E}-5$ \\
\hline 2-methyldecane & $\mathrm{C}_{11} \mathrm{H}_{24}$ & $1198.23,4.449$ & 156.1873 & 1266319 & \\
\hline Azetidine & $\mathrm{C}_{3} \mathrm{H}_{7} \mathrm{~N}$ & $1210.23,3.866$ & 57.0573 & 19609 & \\
\hline $\begin{array}{l}\text { N-Benzyl-N-[(3E)-3,5- } \\
\text { hexadien-1-yl]amine }\end{array}$ & $\mathrm{C}_{13} \mathrm{H}_{19} \mathrm{~N}$ & $1216.23,2.657$ & 189.1512 & 30286 & \\
\hline $\begin{array}{l}\text { 3- } \\
\text { Phenoxytetracyclo[3.2.0.02,7.0 } \\
\text { 4,6]heptane }\end{array}$ & $\mathrm{C}_{13} \mathrm{H}_{12} \mathrm{O}$ & $1222.24,2.641$ & 184.0883 & 16637 & \\
\hline (1-Methylpropyl)cyclohexane & $\mathrm{C}_{10} \mathrm{H}_{20}$ & $1222.24,3.634$ & 140.156 & 73715 & \\
\hline 5-Butylnonane & $\mathrm{C}_{13} \mathrm{H}_{28}$ & $1222.24,4.392$ & 184.2186 & 109565 & \\
\hline Butylcyclohexane & $\mathrm{C}_{10} \mathrm{H}_{20}$ & $1234.24,3.693$ & 140.156 & 726115 & \\
\hline 3-Methyldecane & $\mathrm{C}_{11} \mathrm{H}_{24}$ & $1234.24,4.423$ & 156.1873 & 1009186 & \\
\hline 2,6-Dimethyl-1-octanol & $\mathrm{C}_{10} \mathrm{H}_{22} \mathrm{O} ?$ & $1258.25,3.972$ & 158.1665 & 108369 & $9.75 \mathrm{E}-4$ \\
\hline 1-Ethyl-2-methylbenzene & $\mathrm{C}_{9} \mathrm{H}_{12}$ & $1264.25,2.638$ & 120.0934 & 331698 & \\
\hline 1-Methylpropyl)cyclooctane & $\mathrm{C}_{12} \mathrm{H}_{24}$ & $1276.25,3.965$ & 168.1873 & 397961 & \\
\hline Mesitylene & $\mathrm{C}_{9} \mathrm{H}_{12}$ & $1288.25,2.659$ & 120.0934 & 1026177 & \\
\hline 1-Methyl-1-nitrocyclohexane & $\mathrm{C}_{7} \mathrm{H}_{13} \mathrm{NO}_{2}$ & $1300.26,3.970$ & 143.0941 & 25607 & \\
\hline 1,17-Octadecadiene & $\mathrm{C}_{18} \mathrm{H}_{34}$ & $1312.26,4.299$ & 250.2655 & 48794 & \\
\hline 2,6,10-Trimethyltridecane & $\mathrm{C}_{16} \mathrm{H}_{34}$ & $1312.26,4.703$ & 226.2655 & 86134 & \\
\hline 4,6-Dimethyldecan-1-ol & $\mathrm{C}_{12} \mathrm{H}_{26} \mathrm{O} ?$ & $1318.26,3.944$ & 186.1978 & 179400 & $1.61 \mathrm{E}-3$ \\
\hline Tricosane & $\mathrm{C}_{23} \mathrm{H}_{48}$ & $1330.27,4.667$ & 324.3751 & 152005 & \\
\hline Octadecane & $\mathrm{C}_{18} \mathrm{H}_{38}$ & $1342.27,4.687$ & 254.2968 & 249831 & \\
\hline 1-Ethyl-4-methylbenzene & $\mathrm{C}_{9} \mathrm{H}_{12}$ & $1372.28,2.608$ & 120.0934 & 60924 & \\
\hline $\begin{array}{l}\text { 1-Methyl-3-propyl } \\
\text { cyclohexane }\end{array}$ & $\mathrm{C}_{10} \mathrm{H}_{20}$ & $1384.28,3.907$ & 140.156 & 185975 & \\
\hline Tridecane & $\mathrm{C}_{13} \mathrm{H}_{28}$ & $1390.28,4.706$ & 184.2186 & 1411236 & \\
\hline 2-Methylpropyl)benzene & $\mathrm{C}_{10} \mathrm{H}_{14}$ & $1402.29,2.836$ & 134.109 & 23964 & \\
\hline
\end{tabular}




\begin{tabular}{|c|c|c|c|c|c|}
\hline m-Menthane & $\mathrm{C}_{10} \mathrm{H}_{20}$ & $1414.29,4.011$ & 140.156 & 333766 & \\
\hline Tetradecane & $\mathrm{C}_{14} \mathrm{H}_{30}$ & $1420.29,4.680$ & 198.2342 & 425685 & \\
\hline O-(1-Phenylethyl)acetaldoxime & $\mathrm{C}_{10} \mathrm{H}_{13} \mathrm{NO} ?$ & $1432.3,2.806$ & 163.0992 & 24251 & $2.18 \mathrm{E}-4$ \\
\hline 3,7-Dimethyldecane & $\mathrm{C}_{12} \mathrm{H}_{26}$ & $1444.3,4.649$ & 170.2029 & 522190 & \\
\hline Decahydronaphthalene & $\mathrm{C}_{10} \mathrm{H}_{18}$ & $1468.31,3.343$ & 138.1403 & 254209 & \\
\hline Heptadecane & $\mathrm{C}_{17} \mathrm{H}_{36}$ & $1468.31,4.685$ & 240.2812 & 382716 & \\
\hline $\begin{array}{l}\text { Methyl(1-methylethyl) } \\
\text { benzene }\end{array}$ & $\mathrm{C}_{10} \mathrm{H}_{14}$ & $1492.31,2.777$ & 134.109 & 105588 & \\
\hline $\begin{array}{l}\text { N-(But-2-enoyl)prop-2- } \\
\text { enamide }\end{array}$ & $\mathrm{C}_{7} \mathrm{H}_{11} \mathrm{NO}$ & $1498.32,4.205$ & 125.0835 & 31150 & \\
\hline 4-Methyloctane & $\mathrm{C}_{9} \mathrm{H}_{20}$ & $1498.32,4.588$ & 128.156 & 76087 & \\
\hline $\begin{array}{l}\text { 1-Isopropyl-1- } \\
\text { methylcyclohexane }\end{array}$ & $\mathrm{C}_{10} \mathrm{H}_{20}$ & $1516.32,3.911$ & 140.156 & 64801 & \\
\hline 2-(1-Phenylethyl)phenol & $\mathrm{C}_{14} \mathrm{H}_{14} \mathrm{O}$ ? & $1534.33,4.942$ & 198.1039 & 24740 & $2.22 \mathrm{E}-4$ \\
\hline Dioctyldisulfide & $\mathrm{C}_{16} \mathrm{H}_{34} \mathrm{~S}_{2}$ & $1540.33,4.889$ & 290.2096 & 13230 & \\
\hline 4-Ethyldecane & $\mathrm{C}_{12} \mathrm{H}_{26}$ & $1546.33,4.639$ & 170.2029 & 210017 & \\
\hline 2-Butoxyethanol & $\mathrm{C}_{6} \mathrm{H}_{14} \mathrm{O}_{2}$ & $1552.33,4.936$ & 118.0988 & 75003 & \\
\hline 6-Methylundecane & $\mathrm{C}_{12} \mathrm{H}_{26}$ & $1564.33,4.634$ & 170.2029 & 861108 & \\
\hline $\begin{array}{l}\text { 1,1,3-Trimethyl-3-(2-methyl-2- } \\
\text { propenyl)cyclopentane }\end{array}$ & $\mathrm{C}_{12} \mathrm{H}_{22}$ & $1570.34,4.222$ & 166.1716 & 188177 & \\
\hline 4-Ethyloctane & $\mathrm{C}_{10} \mathrm{H}_{22}$ & $1570.34,4.618$ & 142.1716 & 928014 & \\
\hline 1,2,3-Trimethylbenzene & $\mathrm{C}_{9} \mathrm{H}_{12}$ & $1588.34,2.613$ & 120.0934 & 130610 & \\
\hline 2,2-Dimethylpropane & $\mathrm{C}_{5} \mathrm{H}_{12}$ & $1606.35,4.953$ & 72.0934 & 186871 & \\
\hline Diphenylhexane & $\mathrm{C}_{18} \mathrm{H}_{20}$ & $1642.36,2.799$ & 236.156 & 16949 & \\
\hline 3-Methylundecane & $\mathrm{C}_{12} \mathrm{H}_{26}$ & $1642.36,4.587$ & 170.2029 & 1041652 & \\
\hline $\begin{array}{l}\text { 1-Hydroxymethyl-1,3-dimethyl } \\
\text { cyclopentane }\end{array}$ & $\mathrm{C}_{8} \mathrm{H}_{16} \mathrm{O}$ & $1660.36,4.200$ & 128.1196 & 132418 & \\
\hline 2-Ethyl-1,4-dimethylbenzene & $\mathrm{C}_{10} \mathrm{H}_{14}$ & $1672.37,2.757$ & 134.109 & 416535 & \\
\hline sec-Butylcyclooctane & $\mathrm{C}_{12} \mathrm{H}_{24}$ & $1684.37,4.091$ & 168.1873 & 44417 & \\
\hline $\begin{array}{l}\mathrm{N}-(1 \text {-Adamantyl)oxy-N,N- } \\
\text { dibutylamine }\end{array}$ & $\mathrm{C}_{18} \mathrm{H}_{33} \mathrm{NO} ?$ & $1714.38,3.300$ & 279.2557 & 35520 & $3.19 \mathrm{E}-4$ \\
\hline $\begin{array}{l}\text { 1- } \\
\text { Methyldecahydronaphthalene }\end{array}$ & $\mathrm{C}_{11} \mathrm{H}_{20}$ & $1756.39,3.483$ & 152.156 & 104574 & \\
\hline $\begin{array}{l}\text { 1-(1-Cyclopentenyl)-1- } \\
\text { hydroxy-5,5-dimethyl-6- } \\
\text { hepten-3-one }\end{array}$ & $\mathrm{C}_{14} \mathrm{H}_{22} \mathrm{O}_{2}$ ? & $1768.39,4.359$ & 222.1614 & 27139 & $2.44 \mathrm{E}-4$ \\
\hline Dibutyl sulfite & $\mathrm{C}_{8} \mathrm{H}_{18} \mathrm{O}_{3} \mathrm{~S}$ & $1780.4,4.788$ & 194.0971 & 251745 & \\
\hline 2,4-Dimethyl--1,3-hexanediol & $\mathrm{C}_{8} \mathrm{H}_{18} \mathrm{O}_{2}$ ? & $1786.4,4.021$ & 146.1301 & 34424 & $3.09 \mathrm{E}-4$ \\
\hline
\end{tabular}




\begin{tabular}{|c|c|c|c|c|c|}
\hline 2,6-Dimethylundecane & $\mathrm{C}_{13} \mathrm{H}_{28}$ & $1786.4,4.793$ & 184.2186 & 3261178 & \\
\hline 1-Butyl-2-hexylcyclopropane & $\mathrm{C}_{13} \mathrm{H}_{26}$ & $1798.4,3.999$ & 182.2029 & 16049 & \\
\hline $\begin{array}{l}\text { (2"E)-2'-Methyl-2'-(1",4",4"- } \\
\text { trimethylpent-2"- } \\
\text { enyloxy)propyl } \\
\text { cyclopropanecarboxylate }\end{array}$ & $\mathrm{C}_{16} \mathrm{H}_{28} \mathrm{O}_{3}$ ? & $1804.4,4.405$ & 268.2033 & 46401 & $4.17 \mathrm{E}-4$ \\
\hline 2,3,3-Trimethyl-(Z)- 4-nonene & $\mathrm{C}_{12} \mathrm{H}_{24}$ & $1810.4,4.142$ & 168.1873 & 147898 & \\
\hline Tetraethylbutanedinitrile & $\mathrm{C}_{12} \mathrm{H}_{20} \mathrm{~N}_{2}$ & $1834.41,4.163$ & 192.1621 & 14224 & \\
\hline 1-Methyl-2-pentylcyclohexane & $\mathrm{C}_{12} \mathrm{H}_{24}$ & $1840.41,4.109$ & 168.1873 & 224220 & \\
\hline Heptacosane & $\mathrm{C}_{27} \mathrm{H}_{56}$ & $1846.42,5.020$ & 380.4377 & 31661 & \\
\hline $\begin{array}{l}\text { 1,1'-(1,2-Dimethyl-1,2- } \\
\text { ethanediyl)bis-cyclohexane }\end{array}$ & $\mathrm{C}_{16} \mathrm{H}_{30}$ & $1852.42,4.063$ & 222.2342 & 214903 & \\
\hline $\begin{array}{l}\text { Ethyl 5,7,9-trimethyltridec-3- } \\
\text { enethioate }\end{array}$ & $\mathrm{C}_{18} \mathrm{H}_{34} \mathrm{OS}$ ? & $1864.42,4.359$ & 298.2325 & 66209 & $5.95 \mathrm{E}-4$ \\
\hline 2,3-Dimethylundecane & $\mathrm{C}_{13} \mathrm{H}_{28}$ & $1864.42,4.716$ & 184.2186 & 294724 & \\
\hline $\begin{array}{l}\text { 1-(2,4-Dimethylphenyl)-4- } \\
\text { methyl-1-pentanone }\end{array}$ & $\mathrm{C}_{14} \mathrm{H}_{20} \mathrm{O}$ ? & $1882.43,2.863$ & 204.1509 & 8096 & $7.28-5$ \\
\hline $\begin{array}{l}\text { 1-Methyl-2- } \\
\text { (tricyclo[4.1.0.0(2,7)]hept-1- } \\
\text { yl)benene }\end{array}$ & $\mathrm{C}_{14} \mathrm{H}_{16}$ & $1882.43,2.918$ & 184.1247 & 17101 & \\
\hline 2,9-Dimethyldecane & $\mathrm{C}_{12} \mathrm{H}_{26}$ & $1882.43,4.735$ & 170.2029 & 136149 & \\
\hline 3,4-Dimethylcumene & $\mathrm{C}_{11} \mathrm{H}_{16}$ & $1888.43,2.854$ & 148.1247 & 63166 & \\
\hline 2-Cyclohexyldecane & $\mathrm{C}_{16} \mathrm{H}_{32}$ & $1900.43,4.025$ & 224.2499 & 110054 & \\
\hline 4-Methyldodecane & $\mathrm{C}_{13} \mathrm{H}_{28}$ & $1900.43,4.659$ & 184.2186 & 90938 & \\
\hline $\begin{array}{l}\text { S-Butyl } \\
\text { cyclohexanecarbothioate }\end{array}$ & $\mathrm{C}_{11} \mathrm{H}_{20} \mathrm{OS}$ & $1912.43,4.261$ & 200.1229 & 25938 & \\
\hline 1,2-Diethyl-cis-cyclohexane & $\mathrm{C}_{10} \mathrm{H}_{20}$ & $1930.44,4.312$ & 140.156 & 62526 & \\
\hline Hexadecane & $\mathrm{C}_{16} \mathrm{H}_{34}$ & $1936.44,4.958$ & 226.2655 & 387334 & \\
\hline (2-Methylpropyl)cyclohexane & $\mathrm{C}_{10} \mathrm{H}_{20}$ & $1942.44,3.975$ & 140.156 & 149251 & \\
\hline $\begin{array}{l}\text { 3,3-Dimethylcyclohex-1-en-1- } \\
\text { ylbut-2-enoate }\end{array}$ & $\mathrm{C}_{12} \mathrm{H}_{18} \mathrm{O}_{2}$ ? & $1942.44,4.217$ & 194.1301 & 26303 & $2.36 \mathrm{E}-4$ \\
\hline $\begin{array}{l}2- \\
\text { Methylcyclopentylacetophenon } \\
\text { e }\end{array}$ & $\mathrm{C}_{14} \mathrm{H}_{18} \mathrm{O}$ & $1948.44,2.681$ & 202.1352 & 20636 & \\
\hline 4-Ethylundecane & $\mathrm{C}_{13} \mathrm{H}_{28}$ & $1954.45,4.659$ & 184.2186 & 204820 & \\
\hline 1,2,3,4-Tetramethylbenzene & $\mathrm{C}_{10} \mathrm{H}_{14}$ & $1960.45,2.751$ & 134.109 & 139505 & \\
\hline $\begin{array}{l}\text { 3-Cyclohexyl-(2S-trans)- } \\
\text { oxiranemethanol }\end{array}$ & $\mathrm{C}_{9} \mathrm{H}_{16} \mathrm{O}_{2}$ & $1960.45,3.662$ & 156.1145 & 12543 & \\
\hline $\begin{array}{l}2,6,10,14- \\
\text { Tetramethylhexadecane, }\end{array}$ & $\mathrm{C}_{20} \mathrm{H}_{42}$ & $1960.45,4.932$ & 282.3281 & 47954 & \\
\hline 4-Methylchroman & $\mathrm{C}_{10} \mathrm{H}_{12} \mathrm{O}$ & $1966.45,2.852$ & 148.0883 & 13187 & \\
\hline $\begin{array}{l}\text { 4-Propyl-2,8- } \\
\text { dioxabicyclo[3.3.0]octan-3-one }\end{array}$ & $\mathrm{C}_{9} \mathrm{H}_{14} \mathrm{O}_{3}$ & $1972.45,4.245$ & 170.0937 & 22513 & \\
\hline
\end{tabular}




\begin{tabular}{|c|c|c|c|c|c|}
\hline 6-Methyldodecane & $\mathrm{C}_{13} \mathrm{H}_{28}$ & $1972.45,4.649$ & 184.2186 & 1643386 & \\
\hline Hexadecanoic acid & $\mathrm{C}_{16} \mathrm{H}_{32} \mathrm{O}_{2}$ ? & $1972.45,4.926$ & 256.2397 & 67741 & 6.09 \\
\hline $\begin{array}{l}\text { 3-Ethyl-6-ethylidene-3-methyl- } \\
\text { 1,4-cyclohexadiene }\end{array}$ & $\mathrm{C}_{11} \mathrm{H}_{16}$ & $1984.45,2.874$ & 148.1247 & 23147 & \\
\hline 1-Ethyl-3,5-dimethyl- benzene & $\mathrm{C}_{10} \mathrm{H}_{14}$ & $1996.46,2.721$ & 134.109 & 224148 & \\
\hline 1-(2-Ethylphenyl)ethan-1-one & $\mathrm{C}_{10} \mathrm{H}_{12} \mathrm{O}$ & $1996.46,2.835$ & 148.0883 & 18451 & \\
\hline 2,3,7-Trimethyloctane & $\mathrm{C}_{11} \mathrm{H}_{24}$ & $2002.46,4.947$ & 156.1873 & 1696737 & \\
\hline 2-Methyldodecane & $\mathrm{C}_{13} \mathrm{H}_{28}$ & $2014.46,4.663$ & 184.2186 & 909384 & \\
\hline 2-Methylazetidine & $\mathrm{C}_{4} \mathrm{H}_{9} \mathrm{~N}$ & $2014.46,4.932$ & 71.073 & 54396 & \\
\hline $\begin{array}{l}\text { 1-Methyl-4-(1- } \\
\text { methylpropyl)benzene }\end{array}$ & $\mathrm{C}_{11} \mathrm{H}_{16}$ & $2020.47,2.865$ & 148.1247 & 214683 & \\
\hline Pentylidenecyclohexane & $\mathrm{C}_{11} \mathrm{H}_{20}$ & $2026.47,3.340$ & 152.156 & 23411 & \\
\hline $\begin{array}{l}\text { 1-Ethyl-4-(1- } \\
\text { methylethyl)benzene }\end{array}$ & $\mathrm{C}_{11} \mathrm{H}_{16}$ & $2038.47,2.816$ & 148.1247 & 63926 & \\
\hline Neopentyl 2-oxobutanoate & $\mathrm{C}_{9} \mathrm{H}_{16} \mathrm{O}_{3}$ & $2038.47,4.572$ & 172.1094 & 108730 & \\
\hline 2-Methylpyrrolizidine & $\mathrm{C}_{8} \mathrm{H}_{15} \mathrm{~N}$ & $2050.47,4.188$ & 125.1199 & 25877 & \\
\hline Germacrene & $\mathrm{C}_{15} \mathrm{H}_{30}$ & $2056.48,4.248$ & 210.2342 & 233261 & \\
\hline 1-Methyladamantane & $\mathrm{C}_{11} \mathrm{H}_{18}$ & $2074.48,3.109$ & 150.1403 & 18584 & \\
\hline Hexylcyclohexane & $\mathrm{C}_{12} \mathrm{H}_{24}$ & $2086.48,3.924$ & 168.1873 & 771719 & \\
\hline 2-Methyl-4-hexyn-3-ol & $\mathrm{C}_{7} \mathrm{H}_{12} \mathrm{O}$ & $2086.48,4.180$ & 112.0883 & 107032 & \\
\hline $\begin{array}{l}\text { Tetrahydromethyl-2- } \\
\text { furanpropanoic acid }\end{array}$ & $\mathrm{C}_{8} \mathrm{H}_{14} \mathrm{O}_{3}$ ? & $2104.49,4.781$ & 158.0937 & 32452 & $2.92 \mathrm{E}-4$ \\
\hline 10-Acetoxydecanal & $\mathrm{C}_{12} \mathrm{H}_{22} \mathrm{O}_{3}$ ? & $2110.49,4.202$ & 214.1563 & 223112 & $2.00 \mathrm{E}-3$ \\
\hline $\begin{array}{l}\text { Bis(2-cyclohexyl-1- } \\
\text { methylethyl 1,2-dioxidediazene }\end{array}$ & $\mathrm{C}_{18} \mathrm{H}_{34} \mathrm{~N}_{2} \mathrm{O}_{2}$ ? & $2122.49,4.469$ & 310.2615 & 55201 & $4.96 \mathrm{E}-4$ \\
\hline (1,1-Dimethylpropyl)benzene & $\mathrm{C}_{11} \mathrm{H}_{16}$ & $2128.5,2.849$ & 148.1247 & 73575 & \\
\hline $\begin{array}{l}\text { 2-(3,5-Dimethyl-1-adamantyl)- } \\
\text { 1,2-dicyanoethane }\end{array}$ & $\mathrm{C}_{16} \mathrm{H}_{22} \mathrm{~N}_{2}$ & $2128.5,3.563$ & 242.1778 & 12520 & \\
\hline $\begin{array}{l}\text { 1'-Cyclobutan]-2'-one, } 1,4,4- \\
\text { trimethylspiro[bicyclo[3.2.0]he } \\
\text { pt-6-ene-2 }\end{array}$ & $\mathrm{C}_{13} \mathrm{H}_{18} \mathrm{O}$ & $2134.5,2.847$ & 190.1352 & 23570 & \\
\hline 3-Methyl-, [R-(E)]-4-hexenal & $\mathrm{C}_{7} \mathrm{H}_{12} \mathrm{O}$ & $2134.5,4.115$ & 112.0883 & 84744 & \\
\hline $\begin{array}{l}\text { trans-1-Methyl-2-nonyl- } \\
\text { cyclohexane }\end{array}$ & $\mathrm{C}_{16} \mathrm{H}_{32}$ & $2152.5,4.092$ & 224.2499 & 77812 & \\
\hline $\begin{array}{l}\text { cis,trans-2- } \\
\text { Ethylbicyclo[4.4.0]decane }\end{array}$ & $\mathrm{C}_{12} \mathrm{H}_{22}$ & $2158.5,3.569$ & 166.1716 & 145306 & \\
\hline $\begin{array}{l}\text { 1-Methyl-2-(2'-methylprop-2'- } \\
\text { enyl)cyclododecan-1-ol }\end{array}$ & $\mathrm{C}_{17} \mathrm{H}_{32} \mathrm{O}$ ? & $2164.51,4.390$ & 252.2448 & 20329 & $1.82 \mathrm{E}-4$ \\
\hline $\begin{array}{l}\text { 1,4-Dimethyl-2-(2- } \\
\text { methylpropyl)benzene }\end{array}$ & $\mathrm{C}_{12} \mathrm{H}_{18}$ & $2170.51,2.978$ & 162.1403 & 87696 & \\
\hline 1-Ethyl-2,3-dimethylbenzene & $\mathrm{C}_{10} \mathrm{H}_{14}$ & $2176.51,2.664$ & 134.109 & 37821 & \\
\hline
\end{tabular}




\begin{tabular}{|c|c|c|c|c|c|}
\hline 2-(4'-Methylphenyl)-propanal & $\mathrm{C}_{10} \mathrm{H}_{12} \mathrm{O}$ & $2182.51,2.813$ & 148.0883 & 112253 & \\
\hline Benzyl 4-methylphenyl ketone & $\mathrm{C}_{15} \mathrm{H}_{14} \mathrm{O}$ ? & $2188.51,2.963$ & 210.1039 & 12242 & 1.10 \\
\hline $\begin{array}{l}\text { 2-Methyl-2- } \\
\text { dicyanomethyladamantane }\end{array}$ & $\mathrm{C}_{14} \mathrm{H}_{18} \mathrm{~N}_{2}$ & $2194.51,3.277$ & 214.1465 & 31277 & \\
\hline $\begin{array}{l}\text { 3,4-Trimethyl- } \\
\text { benzeneacetaldehyde }\end{array}$ & $\mathrm{C}_{11} \mathrm{H}_{14} \mathrm{O}$ & $2200.52,2.932$ & 162.1039 & 58723 & \\
\hline (Z)-6-Undecen-3-ol & $\mathrm{C}_{11} \mathrm{H}_{22} \mathrm{O}$ & $2200.52,3.494$ & 170.1665 & 11941 & \\
\hline $\begin{array}{l}\text { Ethyl N-cyano-N- } \\
\text { pentylcarbamate }\end{array}$ & $\mathrm{C}_{9} \mathrm{H}_{16} \mathrm{~N}_{2} \mathrm{O}_{2}$ ? & $2206.52,4.051$ & 184.1206 & 27111 & $2.43 \mathrm{E}-4$ \\
\hline $\begin{array}{l}\text { (2Z,4E)-5-(2'-Furyl)-3- } \\
\text { methylpenta-2,4-dienal }\end{array}$ & $\mathrm{C}_{10} \mathrm{H}_{10} \mathrm{O}_{2}$ & $2212.52,2.898$ & 162.0675 & 11393 & \\
\hline 1-Octadecanol & $\mathrm{C}_{18} \mathrm{H}_{38} \mathrm{O}$ ? & $2218.52,4.040$ & 270.2917 & 22434 & $2.01 \mathrm{E}-4$ \\
\hline 1-Phenyl-(Z)-2-Butene & $\mathrm{C}_{10} \mathrm{H}_{12}$ & $2230.53,2.546$ & 132.0934 & 17652 & \\
\hline $\begin{array}{l}\text { 1-(1-Methyl-1-phenylethyl)-4- } \\
\text { phenyl-1,2,4-triazolidine-3,5- } \\
\text { dione }\end{array}$ & $\mathrm{C}_{17} \mathrm{H}_{17} \mathrm{~N}_{3} \mathrm{O}_{2}$ & $2236.53,2.986$ & 295.1315 & 24655 & \\
\hline 2-Nitrocyclotridecanone & $\mathrm{C}_{13} \mathrm{H}_{23} \mathrm{NO}_{3}$ & $2236.53,4.164$ & 241.1672 & 10269 & \\
\hline $\begin{array}{l}\text { (E)-1,1,2,2-Tetramethyl-3- } \\
\text { oxahepta-4,6-dienyl acrylate }\end{array}$ & $\mathrm{C}_{13} \mathrm{H}_{20} \mathrm{O}_{3}$ & $2236.53,4.354$ & 224.1407 & 26956 & \\
\hline $\begin{array}{l}\text { 3-Oxo-1,3-dihydro-1- } \\
\text { isobenzofuranyl phenylacetate }\end{array}$ & $\mathrm{C}_{16} \mathrm{H}_{12} \mathrm{O}_{4}$ & $2242.53,2.976$ & 268.073 & 16185 & \\
\hline $\begin{array}{l}\text { 2,2,7-Trimethyl-6-octene-3- } \\
\text { one }\end{array}$ & $\mathrm{C}_{11} \mathrm{H}_{20} \mathrm{O}$ & $2254.53,4.335$ & 168.1509 & 59683 & \\
\hline 3,3,4,4-Tetraethylhexane & $\mathrm{C}_{14} \mathrm{H}_{30}$ & $2254.53,4.704$ & 198.2342 & 33497 & \\
\hline 2,6-Nonanedione & $\mathrm{C}_{9} \mathrm{H}_{16} \mathrm{O}_{2}$ & $2254.53,4.935$ & 156.1145 & 19777 & \\
\hline $\begin{array}{l}\text { 4a-Methyl-5,6,7,8-tetrahydro- } \\
\text { 2(4aH)naphthalenone }\end{array}$ & $\mathrm{C}_{11} \mathrm{H}_{14} \mathrm{O}$ & $2260.53,2.982$ & 162.1039 & 11813 & \\
\hline 6,9-Dimethyltetradecane & $\mathrm{C}_{16} \mathrm{H}_{34}$ & $2260.53,4.663$ & 226.2655 & 199591 & \\
\hline $\begin{array}{l}\text { Decahydro-1,1,6-trimethyl } \\
\text { Naphthalene }\end{array}$ & $\mathrm{C}_{13} \mathrm{H}_{24}$ & $2266.54,3.702$ & 180.1873 & 18598 & \\
\hline (E)-5-Heptenenitrile & $\mathrm{C}_{7} \mathrm{H}_{11} \mathrm{~N}$ & $2272.54,3.713$ & 109.0886 & 11814 & \\
\hline $\begin{array}{l}\text { 1-(2',3'-Dihydro-1'-methyl-1H- } \\
\text { inden-3'-yl)-3-hydroxy-3- } \\
\text { methylbutan-1-one }\end{array}$ & $\mathrm{C}_{15} \mathrm{H}_{20} \mathrm{O}_{2}$ ? & $2284.54,2.715$ & 232.1458 & 8436 & $7.59 \mathrm{E}-5$ \\
\hline $\begin{array}{l}\text { 1,2-bis[p-Methylbenzoyloxy- } \\
\text { (p-tolyl)]ethane }\end{array}$ & $\mathrm{C}_{32} \mathrm{H}_{28} \mathrm{O}_{4}$ & $2290.54,2.769$ & 476.1982 & 11987 & $1.07 \mathrm{E}-4$ \\
\hline $\begin{array}{l}\text { 2,2-Dimethyl-4-(methylethyl)- } \\
\text { 2H-imidazole }\end{array}$ & $\mathrm{C}_{8} \mathrm{H}_{14} \mathrm{~N}_{2}$ & $2296.54,4.270$ & 138.1151 & 30186 & \\
\hline $\begin{array}{l}\text { p-Methylphenyl 2-(o- } \\
\text { methylphenyl)-2-propyl } \\
\text { sulfone }\end{array}$ & $\mathrm{C}_{17} \mathrm{H}_{20} \mathrm{O}_{2} \mathrm{~S}$ ? & $2302.55,2.927$ & 288.1179 & 16842 & $1.51 \mathrm{E}-4$ \\
\hline $\begin{array}{l}\text { (5S,7S,9S,E)-S-Ethyl 5,7,9- } \\
\text { trimethyltridec-3-enethioate }\end{array}$ & $\mathrm{C}_{18} \mathrm{H}_{34} \mathrm{OS}$ & $2302.55,4.275$ & 298.2325 & 25611 & \\
\hline $\begin{array}{l}\text { 6-Methyl-1,2,3,4- } \\
\text { tetrahydronaphthalene-1- } \\
\text { methanol }\end{array}$ & $\mathrm{C}_{12} \mathrm{H}_{16} \mathrm{O}$ ? & $2320.55,2.810$ & 176.1196 & 19259 & 1.73 \\
\hline 3-(2-Propenyl)-5-hexen-2-one & $\mathrm{C}_{9} \mathrm{H}_{14} \mathrm{O}$ & $2326.55,4.378$ & 138.1039 & 3408 & \\
\hline 1,2,3,4-Tetrahydronaphthalene & $\mathrm{C}_{10} \mathrm{H}_{12}$ & $2332.55,2.472$ & 132.0934 & 36077 & \\
\hline
\end{tabular}




\begin{tabular}{|c|c|c|c|c|c|}
\hline $\begin{array}{l}\text { Methyl (E)-4,5-epoxyhex-2- } \\
\text { enoate }\end{array}$ & $\mathrm{C}_{7} \mathrm{H}_{10} \mathrm{O}_{3}$ & $2332.55,4.238$ & 142.0624 & 18965 & \\
\hline $\begin{array}{l}\text { (1RS,7RS)-1,4,7,10- } \\
\text { Tetramethyl-2,8- } \\
\text { dioxatricyclo[7.3.0.0(3,7)]dode } \\
\text { ca-3,9-diene }\end{array}$ & $\mathrm{C}_{14} \mathrm{H}_{20} \mathrm{O}_{2}$ & $2338.56,4.304$ & 220.1458 & 21552 & \\
\hline (1,1-Dimethylbutyl)benzene & $\mathrm{C}_{12} \mathrm{H}_{18}$ & $2344.56,2.919$ & 162.1403 & 42347 & \\
\hline 3,3-Dimethylhexane & $\mathrm{C}_{8} \mathrm{H}_{18}$ & $2350.56,4.634$ & 114.1403 & 133244 & \\
\hline $\begin{array}{l}\text { (2E)-1-O-Acetyl-3,7-dimethyl- } \\
\text { 2-octen-1-ol 6,7-epoxide }\end{array}$ & $\mathrm{C}_{12} \mathrm{H}_{20} \mathrm{O}_{3} ?$ & $2356.56,4.597$ & 212.1407 & 21636 & $1.94 \mathrm{E}-4$ \\
\hline $\begin{array}{l}\text { 1-Methyl-3-(2- } \\
\text { methylpropyl)cyclopentane }\end{array}$ & $\mathrm{C}_{10} \mathrm{H}_{20}$ & $2362.56,4.001$ & 140.156 & 82724 & \\
\hline 6-Methyltridecane & $\mathrm{C}_{14} \mathrm{H}_{30}$ & $2368.56,4.602$ & 198.2342 & 1184032 & \\
\hline $\begin{array}{l}\text { Methyl p-(isopropyl)benzyl } \\
\text { sulfoxide }\end{array}$ & $\mathrm{C}_{11} \mathrm{H}_{16} \mathrm{OS}$ & $2374.57,2.870$ & 196.0916 & 32948 & \\
\hline $\begin{array}{l}\text { (E)-4-Methyl-6-(2',5',6',6'- } \\
\text { tetramethylcyclohex-1'- } \\
\text { enyl)hex-3-en-1-ol }\end{array}$ & $\mathrm{C}_{17} \mathrm{H}_{30} \mathrm{O} ?$ & $2374.57,3.687$ & 250.2291 & 32560 & $2.93 \mathrm{E}-4$ \\
\hline 2,5,9-Trimethyldecane & $\mathrm{C}_{13} \mathrm{H}_{28}$ & $2374.57,4.860$ & 184.2186 & 93286 & \\
\hline 5-Methyltridecane & $\mathrm{C}_{14} \mathrm{H}_{30}$ & $2380.57,4.593$ & 198.2342 & 493646 & \\
\hline 3-Butenal & $\mathrm{C}_{4} \mathrm{H}_{6} \mathrm{O}$ & $2380.57,4.855$ & 70.0413 & 18130 & \\
\hline 2,2-Dimethylindan & $\mathrm{C}_{11} \mathrm{H}_{14}$ & $2386.57,2.637$ & 146.109 & 20036 & \\
\hline 2,6-Dimethyl-4-heptanone & $\mathrm{C}_{9} \mathrm{H}_{18} \mathrm{O}$ & $2386.57,4.830$ & 142.1352 & 35844 & \\
\hline 4-Methyltridecane & $\mathrm{C}_{14} \mathrm{H}_{30}$ & $2398.57,4.608$ & 198.2342 & 497242 & \\
\hline $\begin{array}{l}\text { 3-Cyclohexyl-, 3- } \\
\text { cyclohexyldecane }\end{array}$ & $\mathrm{C}_{16} \mathrm{H}_{32}$ & $2410.58,3.885$ & 224.2499 & 17539 & \\
\hline $\begin{array}{l}\text { 1,2,4-Trimethyl-3-nitro-, (2- } \\
\text { endo,3-exo,4-exo)-(.+-.)- } \\
\text { bicyclo[3.3.1]nonan-9-one }\end{array}$ & $\mathrm{C}_{12} \mathrm{H}_{19} \mathrm{NO}_{3}$ ? & $2422.58,3.671$ & 225.1359 & 34141 & 3.07E-4 \\
\hline $\begin{array}{l}\text { (-)-8-Phenylmenthyl 2-phenyl- } \\
\text { 3-ethenylcyclopropane-1- } \\
\text { carboxylate }\end{array}$ & $\mathrm{C}_{28} \mathrm{H}_{34} \mathrm{O}_{2}$ & $2428.58,3.039$ & 402.2553 & 4370 & \\
\hline 2,7,10-Trimethyldodecane & $\mathrm{C}_{15} \mathrm{H}_{32}$ & $2434.58,4.809$ & 212.2499 & 1737835 & \\
\hline $\begin{array}{l}\text { 2,6,10,14- } \\
\text { Tetramethylheptadecane }\end{array}$ & $\mathrm{C}_{21} \mathrm{H}_{44}$ & $2452.59,4.567$ & 296.3438 & 949943 & \\
\hline $\begin{array}{l}\text { 4-[ 1'- } \\
\text { Phenylethenyloxymethyl]pyrid } \\
\text { ine }\end{array}$ & $\mathrm{C}_{14} \mathrm{H}_{13} \mathrm{NO} ?$ & $2482.6,2.922$ & 211.0992 & 6674 & $6.00 \mathrm{E}-5$ \\
\hline $\begin{array}{l}\text { 3-Octyl-1,3-dihydro-1- } \\
\text { isobenzofuranone }\end{array}$ & $\mathrm{C}_{16} \mathrm{H}_{22} \mathrm{O}_{2}$ & $2482.6,3.027$ & 246.1614 & 17182 & \\
\hline $\begin{array}{l}\text { 2-Hydroxy-2-mesityl-2- } \\
\text { phenylacetaldehyde }\end{array}$ & $\mathrm{C}_{10} \mathrm{H}_{11} \mathrm{O}$ & $2494.6,2.973$ & 147.0804 & 10756 & \\
\hline $\begin{array}{l}\text { 1,2,3,4-Tetrahydro-2- } \\
\text { methylnaphthalene }\end{array}$ & $\mathrm{C}_{11} \mathrm{H}_{14}$ & $2500.6,2.588$ & 146.109 & 32086 & \\
\hline $\begin{array}{l}\text { 1-Ethyl-4-(1- } \\
\text { methylpropyl)benzene }\end{array}$ & $\mathrm{C}_{12} \mathrm{H}_{18}$ & $2500.6,2.900$ & 162.1403 & 25151 & \\
\hline $\begin{array}{l}\text { 4-Butyl-2-methylpyrrole-3- } \\
\text { carbonitrile }\end{array}$ & $\mathrm{C}_{10} \mathrm{H}_{14} \mathrm{~N}_{2}$ & $2530.61,2.904$ & 162.1151 & 25813 & \\
\hline $\begin{array}{l}\text { (1S)-4-(1,3-Dithian-2-yl)-3- } \\
\text { methoxy-2-butanone }\end{array}$ & $\mathrm{C}_{9} \mathrm{H}_{16} \mathrm{O}_{2} \mathrm{~S}_{2}$ & $2542.61,2.982$ & 220.0586 & 9551 & \\
\hline
\end{tabular}




\begin{tabular}{|c|c|c|c|c|c|}
\hline 1,1'-Bicyclohexyl & $\mathrm{C}_{12} \mathrm{H}_{22}$ & $2548.62,3.382$ & 166.1716 & 30699 & \\
\hline $\begin{array}{l}\text { 1-Methylcyclohexyl 1-phenyl- } \\
\text { 2-propenyl ketone }\end{array}$ & $\mathrm{C}_{17} \mathrm{H}_{22} \mathrm{O}$ ? & $2578.63,4.100$ & 242.1665 & 15246 & $1.37 \mathrm{E}-4$ \\
\hline $\begin{array}{l}\text { 1-(N-Methoxycarbonyl-2- } \\
\text { pyrrolidinyl)thymine }\end{array}$ & $\mathrm{C}_{11} \mathrm{H}_{15} \mathrm{~N}_{3} \mathrm{O}_{4} ?$ & $2584.63,2.272$ & 253.1057 & 12216 & $1.09 \mathrm{E}-4$ \\
\hline 3-Acetylpropylbenzene & $\mathrm{C}_{11} \mathrm{H}_{14} \mathrm{O}$ ? & $2596.63,2.845$ & 162.1039 & 44232 & $3.98 \mathrm{E}-4$ \\
\hline 4-Methyl-benzaldehyde & $\mathrm{C}_{8} \mathrm{H}_{8} \mathrm{O}$ & $2614.64,2.979$ & 120.057 & 9310 & \\
\hline 5-Ethyl-5-methyl-2-oxepanone & $\mathrm{C}_{9} \mathrm{H}_{16} \mathrm{O}_{2}$ ? & $2614.64,4.333$ & 156.1145 & 38250 & $3.44 \mathrm{E}-4$ \\
\hline Ethenyl cyclohexanoate & $\mathrm{C}_{9} \mathrm{H}_{14} \mathrm{O}_{2}$ ? & $2644.64,4.231$ & 154.0988 & 42195 & $3.79 \mathrm{E}-4$ \\
\hline 2,6,8-Trimethyldecane & $\mathrm{C}_{13} \mathrm{H}_{28}$ & $2644.64,4.646$ & 184.2186 & 214263 & \\
\hline Hexacosane & $\mathrm{C}_{26} \mathrm{H}_{54}$ & $2662.65,4.840$ & 366.422 & 182684 & \\
\hline $\begin{array}{l}\text { (cis)-1-Butyl-2- } \\
\text { undecylcyclopropane }\end{array}$ & $\mathrm{C}_{18} \mathrm{H}_{36}$ & $2668.65,4.249$ & 252.2812 & 63694 & \\
\hline 2,6-Dimethylheptadecane & $\mathrm{C}_{19} \mathrm{H}_{40}$ & $2680.65,4.598$ & 268.3125 & 326491 & \\
\hline Bis(cyclopentylmethyl) ether & $\mathrm{C}_{12} \mathrm{H}_{22} \mathrm{O}$ & $2698.66,4.086$ & 182.1665 & 28595 & \\
\hline $\begin{array}{l}\text { 4-Phenyl-6-thioxo-6,7- } \\
\text { dihydro-8H-pyrimido[1,6- } \\
\text { a]pyrimidin-8-one }\end{array}$ & $\mathrm{C}_{13} \mathrm{H}_{9} \mathrm{~N}_{3} \mathrm{OS}$ ? & $2704.66,3.807$ & 255.0461 & 12939 & $1.16 \mathrm{E}-4$ \\
\hline $\begin{array}{l}\text { 2,3-Dihydro-1,4,7-trimethyl- } \\
\text { 1H-indene }\end{array}$ & $\mathrm{C}_{12} \mathrm{H}_{16}$ & $2716.66,2.727$ & 160.1247 & 17481 & \\
\hline $\begin{array}{l}\text { N-Phenyl-N'-[6-(4(5)- } \\
\text { imidazolyl)hexyl]thiourea } \\
\text { oxalate }\end{array}$ & $\mathrm{C}_{16} \mathrm{H}_{22} \mathrm{~N}_{4} \mathrm{~S} ?$ & $2722.67,3.749$ & 302.156 & 19103 & $1.71 \mathrm{E}-4$ \\
\hline $\begin{array}{l}\text { 4-Hexyl-4-methyl-5- } \\
\text { methylene-1,3-dioxolan-2-one }\end{array}$ & $\mathrm{C}_{11} \mathrm{H}_{18} \mathrm{O}_{3}$ & $2722.67,4.220$ & 198.125 & 17780 & \\
\hline $\begin{array}{l}\text { 2,3-Dihydro-6-methyl-1H- } \\
\text { inden-5-ol }\end{array}$ & $\mathrm{C}_{10} \mathrm{H}_{12} \mathrm{O}$ & $2728.67,2.675$ & 148.0883 & 10573 & \\
\hline $\begin{array}{l}\text { (3,5-cis)-3-Pentyl-5-methyl- } \\
\text { 1,2-oxathiolane-(2,3-trans)-2- } \\
\text { oxide }\end{array}$ & $\mathrm{C}_{9} \mathrm{H}_{18} \mathrm{O}_{2} \mathrm{~S}$ & $2728.67,4.289$ & 190.1022 & 39723 & \\
\hline $\begin{array}{l}\text { 1,2,3,4-Tetrahydro-5- } \\
\text { methylnaphthalene }\end{array}$ & $\mathrm{C}_{11} \mathrm{H}_{14}$ & $2746.67,2.530$ & 146.109 & 79699 & \\
\hline $\begin{array}{l}5,5- \\
\text { Dimethylpentacyclo[5.4.2.0(4, } \\
\text { 11).0(6,8).0(7,11)]tridec-9-en- } \\
\text { 1-ol }\end{array}$ & $\mathrm{C}_{15} \mathrm{H}_{20} \mathrm{O}$ ? & $2752.68,2.931$ & 216.1509 & 17872 & $1.60 \mathrm{E}-4$ \\
\hline $\begin{array}{l}\text { 2,3-Dihydro-1,5,7-trimethyl- } \\
\text { 1H-indene }\end{array}$ & $\mathrm{C}_{12} \mathrm{H}_{16}$ & $2764.68,2.701$ & 160.1247 & 44331 & \\
\hline $\begin{array}{l}\text { 2-Isobutyl-3- } \\
\text { methylcyclopentan-1-one }\end{array}$ & $\mathrm{C}_{10} \mathrm{H}_{18} \mathrm{O}$ & $2764.68,3.984$ & 154.1352 & 22919 & \\
\hline 5-Methyl-tetradecane & $\mathrm{C}_{15} \mathrm{H}_{32}$ & $2764.68,4.536$ & 212.2499 & 493618 & \\
\hline $\begin{array}{l}\text { 2,2',3',5-Tetrahydro-2,3'- } \\
\text { bifuran }\end{array}$ & $\mathrm{C}_{8} \mathrm{H}_{10} \mathrm{O}_{2}$ & $2776.68,4.144$ & 138.0675 & 29795 & \\
\hline 4-Methyltetradecane & $\mathrm{C}_{15} \mathrm{H}_{32}$ & $2782.68,4.546$ & 212.2499 & 458412 & \\
\hline Nonadecane & $\mathrm{C}_{19} \mathrm{H}_{40}$ & $2788.69,4.717$ & 268.3125 & 139772 & \\
\hline 2-Phenyl-3-(p-tolyl)butane & $\mathrm{C}_{17} \mathrm{H}_{20}$ & $2794.69,2.915$ & 224.156 & 8821 & \\
\hline
\end{tabular}




\begin{tabular}{|c|c|c|c|c|c|}
\hline 3-Methyltridecane & $\mathrm{C}_{14} \mathrm{H}_{30}$ & $2800.69,4.541$ & 198.2342 & 886113 & \\
\hline 3-Methyltetradecane & $\mathrm{C}_{15} \mathrm{H}_{32}$ & $2836.7,4.500$ & 212.2499 & 803038 & \\
\hline Isopropoxy-3-methylbenzyl & $\mathrm{C}_{11} \mathrm{H}_{16} \mathrm{O}$ & $2848.7,2.944$ & 164.1196 & 13067 & \\
\hline (E,Z)-7,9-Dodecadien-1-ol & $\mathrm{C}_{12} \mathrm{H}_{22} \mathrm{O}$ ? & $2848.7,3.617$ & 182.1665 & 7397 & $6.65 \mathrm{E}-5$ \\
\hline $\begin{array}{l}\text { 2,5-Dimethylpyrrole-3- } \\
\text { carbonitrile }\end{array}$ & $\mathrm{C}_{7} \mathrm{H}_{8} \mathrm{~N}_{2}$ & $2878.71,2.909$ & 120.0682 & 12613 & \\
\hline $\begin{array}{l}\text { 3,3,12,12-Tetramethyl-1,2- } \\
\text { diaza-1,5,9-cyclododecatriene }\end{array}$ & $\mathrm{C}_{14} \mathrm{H}_{24} \mathrm{~N}_{2}$ & $2878.71,3.705$ & 220.1934 & 5881 & \\
\hline 2-Pent-1-enyltetrahydrofuran & $\mathrm{C}_{9} \mathrm{H}_{16} \mathrm{O}$ & $2878.71,4.153$ & 140.1196 & 50301 & \\
\hline $\begin{array}{l}\text { 1,2,3,4-Tetrahydro-1,4- } \\
\text { dimethylnaphthalene }\end{array}$ & $\mathrm{C}_{12} \mathrm{H}_{16}$ & $2890.71,2.656$ & 160.1247 & 19558 & \\
\hline 1-Phenyl-1-undecyn-5-ol & $\mathrm{C}_{17} \mathrm{H}_{24} \mathrm{O}$ ? & $2890.71,2.757$ & 244.1822 & 10032 & $9.02 \mathrm{E}-5$ \\
\hline $\begin{array}{l}\text { (1R,2R)-1,2-Dihydroxy-3- } \\
\text { cyclohexene }\end{array}$ & $\mathrm{C}_{6} \mathrm{H}_{10} \mathrm{O}_{2}$ & $2896.72,4.110$ & 114.0675 & 41245 & \\
\hline $\begin{array}{l}\text { 1,2,3,4-Tetrahydro-2,7- } \\
\text { dimethylnaphthalene }\end{array}$ & $\mathrm{C}_{12} \mathrm{H}_{16}$ & $2902.72,2.639$ & 160.1247 & 52774 & \\
\hline $\begin{array}{l}\text { (2S, 2R)-2-Isobutyl-3- } \\
\text { methylcyclopentan-1-one }\end{array}$ & $\mathrm{C}_{10} \mathrm{H}_{18} \mathrm{O}$ & $2902.72,4.158$ & 154.1352 & 45784 & \\
\hline $\begin{array}{l}\text { 1,2,3,4-Tetrahydro-6- } \\
\text { methylnaphthalene }\end{array}$ & $\mathrm{C}_{11} \mathrm{H}_{14}$ & $2908.72,2.458$ & 146.109 & 35115 & \\
\hline Octylcyclohexane & $\mathrm{C}_{14} \mathrm{H}_{28}$ & $2908.72,3.893$ & 196.2186 & 802198 & \\
\hline (1E)-1-Hexenylbenzene & $\mathrm{C}_{12} \mathrm{H}_{16}$ & $2914.72,2.650$ & 160.1247 & 16909 & \\
\hline $\begin{array}{l}\text { 1-Ethyl-1,4-dimethyl-trans- } \\
\text { cyclohexane }\end{array}$ & $\mathrm{C}_{10} \mathrm{H}_{20}$ & $2926.73,4.115$ & 140.156 & 179387 & \\
\hline $\begin{array}{l}\text { 1,2,3,4-Tetrahydro-1,1- } \\
\text { dimethylnaphthalene }\end{array}$ & $\mathrm{C}_{12} \mathrm{H}_{16}$ & $2938.73,2.605$ & 160.1247 & 19119 & \\
\hline 1,12-Octadecandiol & $\mathrm{C}_{18} \mathrm{H}_{38} \mathrm{O}_{2}$ ? & $2938.73,3.598$ & 286.2866 & 31470 & $2.83 \mathrm{E}-4$ \\
\hline $\begin{array}{l}\text { (2R,3S)-cis-2,3-Epoxytridecan- } \\
\text { 1-ol }\end{array}$ & $\mathrm{C}_{13} \mathrm{H}_{26} \mathrm{O}_{2}$ ? & $2938.73,4.088$ & 214.1927 & 29820 & $2.68 \mathrm{E}-4$ \\
\hline $\begin{array}{l}\text { (+-)-trans-2-(2,5-Octdiynyl)-3- } \\
\text { undecyloxirane }\end{array}$ & $\mathrm{C}_{21} \mathrm{H}_{34} \mathrm{O}$ & $2950.73,4.017$ & 302.2604 & 76817 & \\
\hline 3-Methyl-1-hexen-5-one & $\mathrm{C}_{7} \mathrm{H}_{12} \mathrm{O}$ & $2974.74,4.052$ & 112.0883 & 34677 & \\
\hline $\begin{array}{l}\text { 2-(trans-2-Methyl-2- } \\
\text { butenoxy)cycloheptene }\end{array}$ & $\mathrm{C}_{12} \mathrm{H}_{20} \mathrm{O}$ & $2980.74,4.056$ & 180.1509 & 23752 & \\
\hline Octyl Allyl Ether & $\mathrm{C}_{11} \mathrm{H}_{22} \mathrm{O}$ & $2980.74,4.545$ & 170.1665 & 32222 & \\
\hline $\begin{array}{l}\text { 3',3',5'-Trimethylcyclohexyl } \\
\text { (cis)-(3- } \\
\text { thienylglyoxyl)carboxylate }\end{array}$ & $\mathrm{C}_{15} \mathrm{H}_{20} \mathrm{O}_{3} \mathrm{~S}$ ? & $2986.74,4.002$ & 280.1128 & 23912 & $2.15 \mathrm{E}-4$ \\
\hline 3-Ethyloctane & $\mathrm{C}_{10} \mathrm{H}_{22}$ & $2992.74,4.599$ & 142.1716 & 85567 & \\
\hline $\begin{array}{l}\text { o-(4-Ethylbenzoyl)-o'-(3- } \\
\text { methylbenzoyl)-1,3- } \\
\text { benzenediol }\end{array}$ & $\mathrm{C}_{23} \mathrm{H}_{20} \mathrm{O}_{4}$ ? & $3004.75,2.868$ & 360.1356 & 17787 & $1.60 \mathrm{E}-4$ \\
\hline 1-Methylnaphthalene & $\mathrm{C}_{11} \mathrm{H}_{10}$ & $3022.75,2.319$ & 142.0777 & 63818 & \\
\hline 1-Isocyanobutane & $\mathrm{C}_{5} \mathrm{H}_{9} \mathrm{~N}$ & $3028.75,4.099$ & 83.073 & 21097 & \\
\hline
\end{tabular}




\begin{tabular}{|c|c|c|c|c|c|}
\hline $\begin{array}{l}\text { (R)-5-(1-Decenyl)-2- } \\
\text { oxotetrahydrofuran }\end{array}$ & $\mathrm{C}_{14} \mathrm{H}_{24} \mathrm{O}_{2}$ & $3058.76,4.009$ & 224.1771 & 19883 & \\
\hline 2-Isononenal & $\mathrm{C}_{9} \mathrm{H}_{16} \mathrm{O}$ & $3058.76,4.175$ & 140.1196 & 17807 & \\
\hline $\begin{array}{l}\text { (6R,12R)-6,12- } \\
\text { Dimethylpentadec-1-ene }\end{array}$ & $\mathrm{C}_{17} \mathrm{H}_{34}$ & $3070.77,3.712$ & 238.2655 & 12897 & \\
\hline $\begin{array}{l}\text { (3R,4S,5R,7R)-3,5,7- } \\
\text { Trimethyl-1-nonen-4-ol }\end{array}$ & $\mathrm{C}_{12} \mathrm{H}_{24} \mathrm{O}$ ? & $3112.78,4.169$ & 184.1822 & 43711 & 3.93E-4 \\
\hline 2,4-Dimethyl-3-pentanone & $\mathrm{C}_{7} \mathrm{H}_{14} \mathrm{O}$ & $3124.78,4.696$ & 114.1039 & 76365 & \\
\hline 1,1'-Bicyclooctyl & $\mathrm{C}_{16} \mathrm{H}_{30}$ & $3136.79,4.146$ & 222.2342 & 48000 & \\
\hline 4-Methylpentadecane & $\mathrm{C}_{16} \mathrm{H}_{34}$ & $3148.79,4.474$ & 226.2655 & 405045 & \\
\hline $\begin{array}{l}\text { 1-Methyl-3- } \\
\text { (phenylsulfonyl)pyrrolidine }\end{array}$ & $\mathrm{C}_{11} \mathrm{H}_{15} \mathrm{NO}_{2} \mathrm{~S} ?$ & $3166.79,3.904$ & 225.0818 & 29920 & 2.69E-4 \\
\hline 2-Methylnonadecane & $\mathrm{C}_{20} \mathrm{H}_{42}$ & $3166.79,4.464$ & 282.3281 & 762289 & \\
\hline 2,6-dimethyloctadecane & $\mathrm{C}_{20} \mathrm{H}_{42}$ & $3202.8,4.423$ & 282.3281 & 679061 & \\
\hline $\begin{array}{l}\text { 4,5-trans-4-(2-Methylpropyl)- } \\
\text { 5-ethenyl-1,3-dioxolan-2-one }\end{array}$ & $\mathrm{C}_{9} \mathrm{H}_{14} \mathrm{O}_{3}$ & $3220.81,4.165$ & 170.0937 & 14474 & \\
\hline 1,2-Dibutyl-cis-cyclopropane & $\mathrm{C}_{11} \mathrm{H}_{22}$ & $3232.81,4.115$ & 154.1716 & 35746 & \\
\hline trans-1,2-Dibutyl-cyclopropane & $\mathrm{C}_{11} \mathrm{H}_{22}$ & $3250.82,4.094$ & 154.1716 & 57260 & \\
\hline $\begin{array}{l}\text { 1,2,3,4-Tetrahydro-5,6- } \\
\text { dimethylnaphthalene }\end{array}$ & $\mathrm{C}_{12} \mathrm{H}_{16}$ & $3268.82,2.526$ & 160.1247 & 76855 & \\
\hline Heptyl propyl oxaloate & $\mathrm{C}_{12} \mathrm{H}_{22} \mathrm{O}_{4}$ ? & $3268.82,4.549$ & 230.1513 & 91456 & 8.23E-4 \\
\hline 1-Cyclohexyl-3-methylbenzene & $\mathrm{C}_{13} \mathrm{H}_{18}$ & $3286.83,2.658$ & 174.1403 & 24025 & \\
\hline n-Nonylcyclohexane & $\mathrm{C}_{15} \mathrm{H}_{30}$ & $3286.83,3.862$ & 210.2342 & 711824 & \\
\hline $\begin{array}{l}\text { 2-Ethyl-2,4,5-trimethyl-2,5- } \\
\text { dihydro-1H-imidazole }\end{array}$ & $\mathrm{C}_{8} \mathrm{H}_{16} \mathrm{~N}_{2}$ & $3286.83,4.068$ & 140.1308 & 16133 & \\
\hline $\begin{array}{l}\text { (Tetrahydro-2-furanyl)methyl } \\
\text { butanoate }\end{array}$ & $\mathrm{C}_{9} \mathrm{H}_{16} \mathrm{O}_{3}$ ? & $3322.84,4.485$ & 172.1094 & 146973 & $1.32 \mathrm{E}-3$ \\
\hline 3-Ethylcosane & $\mathrm{C}_{22} \mathrm{H}_{46}$ & $3334.84,4.692$ & 310.3594 & 219953 & \\
\hline 1,2-Epoxy-3-tetradecanol & $\mathrm{C}_{14} \mathrm{H}_{28} \mathrm{O}_{2}$ & $3370.85,4.001$ & 228.2084 & 198247 & \\
\hline 1,7-Dimethylnaphthalene & $\mathrm{C}_{12} \mathrm{H}_{12}$ & $3418.87,2.386$ & 156.0934 & 95308 & \\
\hline 2-Cyclohexyloxirane & $\mathrm{C}_{8} \mathrm{H}_{14} \mathrm{O}$ & $3418.87,4.067$ & 126.1039 & 1549 & \\
\hline 2-Methyloct-3-enal & $\mathrm{C}_{9} \mathrm{H}_{16} \mathrm{O}$ & $3418.87,4.161$ & 140.1196 & 13069 & \\
\hline 2,6,10-Trimethylpentadecane & $\mathrm{C}_{18} \mathrm{H}_{38}$ & $3418.87,4.670$ & 254.2968 & 2218430 & \\
\hline 3-Acetoxy-1H-pyrrole & $\mathrm{C}_{6} \mathrm{H}_{7} \mathrm{NO}_{2}$ & $3436.87,4.123$ & 125.0471 & 40434 & \\
\hline $\begin{array}{l}\text { [3-(Ethylthio)-1-methyl-1- } \\
\text { butenyl]benzene }\end{array}$ & $\mathrm{C}_{13} \mathrm{H}_{18} \mathrm{~S}$ & $3442.87,2.470$ & 206.1124 & 5737 & \\
\hline 2,7-Dimethylundecane & $\mathrm{C}_{13} \mathrm{H}_{28}$ & $3478.88,4.382$ & 184.2186 & 426023 & \\
\hline 2,5-Dimethylheptane & $\mathrm{C}_{9} \mathrm{H}_{20}$ & $3490.89,4.607$ & 128.156 & 67846 & \\
\hline
\end{tabular}




\begin{tabular}{|c|c|c|c|c|c|}
\hline 4-Methylhexadecane & $\mathrm{C}_{17} \mathrm{H}_{36}$ & $3496.89,4.418$ & 240.2812 & 337368 & \\
\hline $\begin{array}{l}\text { 4-(2- } \\
\text { Tetrahydropyranyl)oxybutanal }\end{array}$ & $\mathrm{C}_{9} \mathrm{H}_{16} \mathrm{O}_{3}$ & $3496.89,4.807$ & 172.1094 & 66789 & \\
\hline 1,8-Dimethylnaphthalene & $\mathrm{C}_{12} \mathrm{H}_{12}$ & $3502.89,2.350$ & 156.0934 & 35917 & \\
\hline $\begin{array}{l}\text { Neopentyl 2,2- } \\
\text { dimethylbutanoate }\end{array}$ & $\mathrm{C}_{11} \mathrm{H}_{22} \mathrm{O}_{2}$ ? & $3502.89,4.377$ & 186.1614 & 47433 & $4.26 \mathrm{E}-4$ \\
\hline 2-Methyleicosane & $\mathrm{C}_{21} \mathrm{H}_{44}$ & $3508.89,4.428$ & 296.3438 & 601850 & \\
\hline $\begin{array}{l}\text { 3,7-Dimethyl-1H-Indene-5- } \\
\text { carboxaldehyde }\end{array}$ & $\mathrm{C}_{12} \mathrm{H}_{12} \mathrm{O}$ & $3514.89,2.488$ & 172.0883 & 10762 & \\
\hline 3-Butene-1,2-diol & $\mathrm{C}_{4} \mathrm{H}_{8} \mathrm{O}_{2}$ & $3514.89,4.789$ & 88.0519 & 46406 & \\
\hline $\begin{array}{l}\text { 2'-Methylspiro[cyclopropane- } \\
1,1^{\prime} \text {-ind-2'-ene] }\end{array}$ & $\mathrm{C}_{12} \mathrm{H}_{12}$ & $3520.9,2.335$ & 156.0934 & 86239 & \\
\hline $\begin{array}{l}\text { 3-(2- } \\
\text { Methylphenyl)cyclohexene }\end{array}$ & $\mathrm{C}_{13} \mathrm{H}_{16}$ & $3520.9,2.477$ & 172.1247 & 14387 & \\
\hline $\begin{array}{l}\text { 1,4-bis( Isopropylamino) } \\
\text { anthraquinone }\end{array}$ & $\mathrm{C}_{20} \mathrm{H}_{20} \mathrm{~N}_{2} \mathrm{O}_{2}$ & $3550.9,4.047$ & 320.1519 & 17798 & \\
\hline 3-Methylhexadecane & $\mathrm{C}_{17} \mathrm{H}_{36}$ & $3550.9,4.356$ & 240.2812 & 515320 & \\
\hline $\begin{array}{l}\text { 1-Methyl-2,3-dihydro-4- } \\
\text { pyridone }\end{array}$ & $\mathrm{C}_{6} \mathrm{H}_{9} \mathrm{NO}$ & $3556.91,3.830$ & 111.0679 & 16804 & \\
\hline 1-Ethylnaphthalene & $\mathrm{C}_{12} \mathrm{H}_{12}$ & $3598.92,2.331$ & 156.0934 & 12993 & \\
\hline Tetradecylcyclooctane & $\mathrm{C}_{22} \mathrm{H}_{44}$ & $3604.92,4.118$ & 308.3438 & 143286 & \\
\hline 2,2-Dimethylbutane & $\mathrm{C}_{6} \mathrm{H}_{14}$ & $3664.94,4.487$ & 86.109 & 151737 & \\
\hline Cyclopentanodec-5-ene & $\mathrm{C}_{15} \mathrm{H}_{28}$ & $3700.95,3.946$ & 208.2186 & 37902 & \\
\hline $\begin{array}{l}\text { (trans)-1-Butyl-2- } \\
\text { undecylcyclopropane }\end{array}$ & $\mathrm{C}_{18} \mathrm{H}_{36}$ & $3718.95,3.973$ & 252.2812 & 247026 & \\
\hline 6-Hydroxy-3-hexanone & $\mathrm{C}_{6} \mathrm{H}_{12} \mathrm{O}_{2}$ & $3754.96,4.566$ & 116.0832 & 59749 & \\
\hline 2-(1-Methylethyl)naphthalene & $\mathrm{C}_{13} \mathrm{H}_{14}$ & $3766.97,2.420$ & 170.109 & 14213 & \\
\hline $\begin{array}{l}\text { 7-Phenyltetracyclo } \\
{[4.1 .0 .02,4.03,5] \text { heptane }}\end{array}$ & $\mathrm{C}_{13} \mathrm{H}_{12}$ & $3772.97,2.282$ & 168.0934 & 19338 & \\
\hline $\begin{array}{l}\text { 3-Methyl-1-[(1H)-1,2,4-triazol- } \\
\text { 1-yl]butan-2-one }\end{array}$ & $\mathrm{C}_{7} \mathrm{H}_{11} \mathrm{~N}_{3} \mathrm{O}$ & $3772.97,3.887$ & 153.0897 & 30038 & \\
\hline $\begin{array}{l}\text { 1-Methyl-4-(1-methylethyl)- } \\
\text { trans-cyclohexane }\end{array}$ & $\mathrm{C}_{10} \mathrm{H}_{20}$ & $3790.97,3.965$ & 140.156 & 137464 & \\
\hline 3,4-Dimethyl-4H-1,2,4-triazole & $\mathrm{C}_{4} \mathrm{H}_{7} \mathrm{~N}_{3}$ & $3796.97,4.018$ & 97.0634 & 50502 & \\
\hline 4-Methylheptadecane & $\mathrm{C}_{18} \mathrm{H}_{38}$ & $3826.98,4.346$ & 254.2968 & 337989 & \\
\hline 2,3-Epoxytridecan-1-ol & $\mathrm{C}_{13} \mathrm{H}_{26} \mathrm{O}_{2}$ ? & $3832.99,3.896$ & 214.1927 & 27297 & $2.45 \mathrm{E}-4$ \\
\hline $\begin{array}{l}\text { 1,2,2,3,3,4,4,5-Octamethyl-6- } \\
\text { oxabicyclo[3.1.0]hexane }\end{array}$ & $\mathrm{C}_{13} \mathrm{H}_{24} \mathrm{O}$ & $3838.99,4.053$ & 196.1822 & 5768 & \\
\hline 8-Heptylpentadecane & $\mathrm{C}_{22} \mathrm{H}_{46}$ & $3838.99,4.402$ & 310.3594 & 514166 & \\
\hline 2-Isocyano-2-methylpropane & $\mathrm{C}_{5} \mathrm{H}_{9} \mathrm{~N}$ & $3844.99,3.892$ & 83.073 & 47767 & \\
\hline 5-Methylhept-2-yn-4-ol & $\mathrm{C}_{8} \mathrm{H}_{14} \mathrm{O}$ ? & $3844.99,3.948$ & 126.1039 & 71078 & $6.39 \mathrm{E}-4$ \\
\hline
\end{tabular}




\begin{tabular}{|c|c|c|c|c|c|}
\hline 4,6,8-Trimethylazulene & $\mathrm{C}_{13} \mathrm{H}_{14}$ & $3869,2.386$ & 170.109 & 42518 & \\
\hline $\begin{array}{l}\text { Methyl 3-(p- } \\
\text { nitrophenyl)bicyclo[1.1.1]pent } \\
\text { ane-1-carboxylate }\end{array}$ & $\mathrm{C}_{13} \mathrm{H}_{13} \mathrm{NO}_{4}$ ? & $3875,2.490$ & 247.0839 & 18964 & $1.70 \mathrm{E}-4$ \\
\hline 4-tert-Butylhex-1-ene & $\mathrm{C}_{10} \mathrm{H}_{20}$ & $3875,4.320$ & 140.156 & 157992 & \\
\hline 1,6,7-Trimethylnaphthalene & $\mathrm{C}_{13} \mathrm{H}_{14}$ & $3881,2.406$ & 170.109 & 54128 & \\
\hline $\begin{array}{l}\text { 1-(3-Methyl-2-butenyl)-2- } \\
\text { hexylcyclopropane }\end{array}$ & $\mathrm{C}_{14} \mathrm{H}_{26}$ & $3887,3.837$ & 194.2029 & 14376 & \\
\hline $\begin{array}{l}\text { Methyl (1S,2R,3S,4R)-3-[(4S)- } \\
\text { 4-(2,2-dimethyl-1,3- } \\
\text { dioxolo)]bicyclo[2.2.1]-5- } \\
\text { hepten-2-ylcarboxylate } \\
\end{array}$ & $\mathrm{C}_{14} \mathrm{H}_{20} \mathrm{O}_{4}$ ? & $3929.01,3.980$ & 252.1356 & 10737 & $9.66 \mathrm{E}-5$ \\
\hline 1,6,7-Trimethylnaphthalene & $\mathrm{C}_{13} \mathrm{H}_{14}$ & $3965.02,2.393$ & 170.109 & 54631 & \\
\hline 7-Oxatridecane & $\mathrm{C}_{12} \mathrm{H}_{26} \mathrm{O}$ & $3989.03,4.422$ & 186.1978 & 45463 & \\
\hline $\begin{array}{l}\text { (Z)-2,2,3,5,5-Pentamethyl-3- } \\
\text { hexene }\end{array}$ & $\mathrm{C}_{11} \mathrm{H}_{22}$ & $4055.05,3.921$ & 154.1716 & 61954 & \\
\hline n-Decyl prop-2-ynoate & $\mathrm{C}_{13} \mathrm{H}_{22} \mathrm{O}_{2}$ ? & $4061.05,3.971$ & 210.1614 & 48716 & $4.38 \mathrm{E}-4$ \\
\hline n-Cetyl thiocyanate & $\mathrm{C}_{17} \mathrm{H}_{33} \mathrm{NS} ?$ & $4067.05,4.340$ & 283.2328 & 95651 & $8.60 \mathrm{E}-4$ \\
\hline 3,4'-Dimethyl-1,1'-biphenyl & $\mathrm{C}_{14} \mathrm{H}_{14}$ & $4121.07,2.311$ & 182.109 & 22041 & \\
\hline 3,3'-Dimethylbiphenyl & $\mathrm{C}_{14} \mathrm{H}_{14}$ & $4163.08,2.313$ & 182.109 & 18660 & \\
\hline $\begin{array}{l}\text { (4E)-2,7-Dimethyl-4-octene- } \\
\text { 3,6-dione }\end{array}$ & $\mathrm{C}_{10} \mathrm{H}_{16} \mathrm{O}_{2}$ & $4181.09,4.249$ & 168.1145 & 42423 & \\
\hline 2-Methyloctadecane & $\mathrm{C}_{19} \mathrm{H}_{40}$ & $4193.09,4.279$ & 268.3125 & 410169 & \\
\hline $\begin{array}{l}\text { 2-(1,3-Dioxolan-2-yl)-1-(2- } \\
\text { furyl)ethene }\end{array}$ & $\mathrm{C}_{9} \mathrm{H}_{10} \mathrm{O}_{3}$ & $4211.09,4.396$ & 166.0624 & 99070 & \\
\hline 2,4,4-Trimethylhexane & $\mathrm{C}_{9} \mathrm{H}_{20}$ & $4235.1,4.383$ & 128.156 & 56630 & \\
\hline $\begin{array}{l}\text { 7-Methylbicyclo[3.2.1]octan-1- } \\
\text { ol }\end{array}$ & $\mathrm{C}_{9} \mathrm{H}_{16} \mathrm{O}$ & $4265.11,3.931$ & 140.1196 & 25243 & \\
\hline $\begin{array}{l}\text { 2,6-Dimethylcyclohexane-1- } \\
\text { (cis)-carbaldehyde }\end{array}$ & $\mathrm{C}_{9} \mathrm{H}_{16} \mathrm{O}$ & $4265.11,4.101$ & 140.1196 & 32116 & \\
\hline 2-Cyanocyclohexanol & $\mathrm{C}_{7} \mathrm{H}_{11} \mathrm{NO}$ & $4331.13,4.316$ & 125.0835 & 59362 & \\
\hline Bicyclo[4.3.1]deca-1-ol & $\mathrm{C}_{10} \mathrm{H}_{18} \mathrm{O}$ ? & $4343.13,3.858$ & 154.1352 & 11229 & $1.01 \mathrm{E}-4$ \\
\hline $\begin{array}{l}\text { Cyclohexylmethyl octadecyl } \\
\text { sulfurous acid ester }\end{array}$ & $\mathrm{C}_{25} \mathrm{H}_{50} \mathrm{O}_{3} \mathrm{~S}$ ? & $4415.15,3.903$ & 430.3475 & 283235 & $2.54 \mathrm{E}-3$ \\
\hline $\begin{array}{l}\text { 2,4-Dimethyl-1-methylethyl } \\
\text { ester, (S)-pentanoic acid }\end{array}$ & $\mathrm{C}_{10} \mathrm{H}_{20} \mathrm{O}_{2} ?$ & $4415.15,4.442$ & 172.1458 & 46722 & $4.20 \mathrm{E}-4$ \\
\hline $\begin{array}{l}\text { Hexyl octyl ester sulfurous } \\
\text { acid }\end{array}$ & $\mathrm{C}_{14} \mathrm{H}_{30} \mathrm{O}_{3} \mathrm{~S}$ ? & $4421.15,4.299$ & 278.191 & 124949 & $1.12 \mathrm{E}-3$ \\
\hline 2,5,9-Trimethyldecane & $\mathrm{C}_{13} \mathrm{H}_{28}$ & $4427.16,4.585$ & 184.2186 & 61920 & \\
\hline 4-Methylhexanoic acid & $\mathrm{C}_{7} \mathrm{H}_{14} \mathrm{O}_{2}$ ? & $4439.16,4.585$ & 130.0988 & 51375 & 4.62E-4 \\
\hline Eicosane & $\mathrm{C}_{20} \mathrm{H}_{42}$ & $4451.16,4.583$ & 282.3281 & 129887 & \\
\hline 3,8-Dimethylundecane & $\mathrm{C}_{13} \mathrm{H}_{28}$ & $4493.17,4.248$ & 184.2186 & 308602 & \\
\hline
\end{tabular}




\begin{tabular}{|c|c|c|c|c|c|}
\hline $\begin{array}{l}\text { 1-Propanoyl-2- } \\
\text { butanoylethylene }\end{array}$ & $\mathrm{C}_{9} \mathrm{H}_{14} \mathrm{O}_{2}$ & $4505.18,4.030$ & 154.0988 & 41660 & \\
\hline $\begin{array}{l}\text { 3-(1,1-Dimethylethoxy)-1- } \\
\text { propene }\end{array}$ & $\mathrm{C}_{7} \mathrm{H}_{14} \mathrm{O}$ & $4517.18,4.336$ & 114.1039 & 77327 & \\
\hline 2-Methoxy-1,4-pentadiene & $\mathrm{C}_{6} \mathrm{H}_{10} \mathrm{O}$ & $4529.18,4.323$ & 98.0726 & 15074 & \\
\hline 1-Deuteriocyclohexene & $\mathrm{C}_{6} \mathrm{H}_{9} \mathrm{D}$ & $4601.21,3.796$ & 83.084 & 24572 & \\
\hline $\begin{array}{l}\text { Tetrahydroacetate-2- } \\
\text { furanmethanol }\end{array}$ & $\mathrm{C}_{7} \mathrm{H}_{12} \mathrm{O}_{3}$ & $4637.22,4.277$ & 144.0781 & 42654 & \\
\hline $\begin{array}{l}\text { 2-(2,2-Dimethylpropylidene)- } \\
\text { 1,3-cyclohexanedione }\end{array}$ & $\mathrm{C}_{11} \mathrm{H}_{16} \mathrm{O}_{2}$ ? & $4649.22,2.241$ & 180.1145 & 17266 & $1.55 \mathrm{E}-4$ \\
\hline $\begin{array}{l}\text { 3-Hydroxy-2,7,7-trimethyl-1,8- } \\
\text { nonadien-5-one }\end{array}$ & $\mathrm{C}_{12} \mathrm{H}_{20} \mathrm{O}_{2}$ ? & $4661.22,3.908$ & 196.1458 & 40285 & $3.62 \mathrm{E}-4$ \\
\hline $\begin{array}{l}\text { [[2-(2-Propenyloxy)-1- } \\
\text { propenyl]sulfonyl]-(E)- } \\
\text { benzene }\end{array}$ & $\mathrm{C}_{12} \mathrm{H}_{14} \mathrm{O}_{3} \mathrm{~S}$ ? & $4667.22,3.931$ & 238.0658 & 76846 & $6.91 \mathrm{E}-4$ \\
\hline 10-Methyleicosane & $\mathrm{C}_{21} \mathrm{H}_{44}$ & $4673.23,4.263$ & 296.3438 & 545922 & \\
\hline 3-Ethyl-3-methylhexane & $\mathrm{C}_{9} \mathrm{H}_{20}$ & $4697.23,4.237$ & 128.156 & 142061 & \\
\hline Heneicosane & $\mathrm{C}_{21} \mathrm{H}_{44}$ & $4739.25,4.269$ & 296.3438 & 577749 & \\
\hline $\begin{array}{l}\text { 2-Methoxy-1-(1H-1,2,3- } \\
\text { triazol-4-yl)ethylamine }\end{array}$ & $\mathrm{C}_{5} \mathrm{H}_{10} \mathrm{~N}_{4} \mathrm{O}$ & $4775.26,3.822$ & 142.0849 & 55364 & \\
\hline $\begin{array}{l}2,6,10,15- \\
\text { Tetramethylheptadecane }\end{array}$ & $\mathrm{C}_{21} \mathrm{H}_{44}$ & $4775.26,4.238$ & 296.3438 & 304181 & \\
\hline (E)-Deca-1,6-dien-3-one & $\mathrm{C}_{10} \mathrm{H}_{16} \mathrm{O}$ & $4823.27,4.333$ & 152.1196 & 45544 & \\
\hline $\begin{array}{l}\text { 2-Ethyl-6- } \\
\text { oxabicyclo[3.1.0]hexan-2-ol }\end{array}$ & $\mathrm{C}_{7} \mathrm{H}_{12} \mathrm{O}_{2}$ & $4913.3,4.253$ & 128.0832 & 22538 & \\
\hline Undecylcyclohexane & $\mathrm{C}_{17} \mathrm{H}_{34}$ & $4925.3,3.744$ & 238.2655 & 251720 & \\
\hline $\begin{array}{l}\text { 5-Methyl-6-carbethoxy-2- } \\
\text { oxabicyclo[3.1.0]hexane }\end{array}$ & $\mathrm{C}_{9} \mathrm{H}_{14} \mathrm{O}_{3}$ ? & $4949.31,3.934$ & 170.0937 & 45322 & 4.07E-4 \\
\hline $\begin{array}{l}\text { 2,4,9,11-Tetramethyldodeca- } \\
\text { 5,7-diyne-4,9-diol }\end{array}$ & $\mathrm{C}_{16} \mathrm{H}_{26} \mathrm{O}_{2}$ ? & $4961.31,4.246$ & 250.1927 & 89912 & 8.09E-4 \\
\hline 9-Hydroxyinalool & $\mathrm{C}_{10} \mathrm{H}_{18} \mathrm{O}_{2}$ ? & $5003.32,4.243$ & 170.1301 & 95522 & $8.59 \mathrm{E}-4$ \\
\hline $\begin{array}{l}\text { Tert-bytyl (2E)-penta-2,4- } \\
\text { dienylcarbamoate }\end{array}$ & $\mathrm{C}_{10} \mathrm{H}_{17} \mathrm{NO}_{2} ?$ & $5009.32,4.460$ & 183.1254 & 55333 & 4.97E-4 \\
\hline 11-Decyldocosane & $\mathrm{C}_{32} \mathrm{H}_{66}$ & $5015.32,4.258$ & 450.5159 & 347597 & \\
\hline 3-Amino-5-nitro-1H-indazole & $\mathrm{C}_{7} \mathrm{H}_{6} \mathrm{~N}_{4} \mathrm{O}_{2}$ & $5027.33,2.132$ & 178.0485 & 10655 & \\
\hline $\begin{array}{l}\text { 2,2,4,5-Tetramethylhex-5-en- } \\
\text { 3-one }\end{array}$ & $\mathrm{C}_{10} \mathrm{H}_{14} \mathrm{O}$ & $5063.34,4.288$ & 150.1039 & 33317 & \\
\hline 2,2,4-Trimethyl-2H-imidazole & $\mathrm{C}_{6} \mathrm{H}_{10} \mathrm{~N}_{2}$ & $5075.34,3.968$ & 110.0838 & 38105 & \\
\hline 2-Methyldihydrothiophene & $\mathrm{C}_{5} \mathrm{H}_{8} \mathrm{~S}$ & $5093.35,4.302$ & 100.0341 & 30150 & \\
\hline (E)-6-Tosyl-4-nonene & $\mathrm{C}_{16} \mathrm{H}_{24} \mathrm{O}_{2} \mathrm{~S} ?$ & $5153.36,4.047$ & 280.1492 & 15290 & $1.37 \mathrm{E}-4$ \\
\hline 1-Hexadecene & $\mathrm{C}_{16} \mathrm{H}_{32}$ & $5171.37,3.801$ & 224.2499 & 23230 & \\
\hline Eicosylcyclohexane & $\mathrm{C}_{26} \mathrm{H}_{52}$ & $5201.38,3.754$ & 364.4064 & 193321 & \\
\hline $\begin{array}{l}\text { 1-Oxa-spiro[5.2]octane-2- } \\
\text { carbaldehyde }\end{array}$ & $\mathrm{C}_{8} \mathrm{H}_{12} \mathrm{O}_{2}$ & $5219.38,3.881$ & 140.0832 & 47417 & \\
\hline
\end{tabular}




\begin{tabular}{|c|c|c|c|c|c|}
\hline $\begin{array}{l}\text { 2,2,3,4,5,5-Hexamethyl- } \\
\text { (meso)hexane }\end{array}$ & $\mathrm{C}_{12} \mathrm{H}_{26}$ & $5249.39,4.235$ & 170.2029 & 103187 & \\
\hline n-Octyl ethynyl ether & $\mathrm{C}_{10} \mathrm{H}_{18} \mathrm{O}$ & $5267.4,4.223$ & 154.1352 & 112975 & \\
\hline 1-Ethyl-1H-1,2,4-triazole & $\mathrm{C}_{4} \mathrm{H}_{7} \mathrm{~N}_{3}$ & $5273.4,3.868$ & 97.0634 & 80146 & \\
\hline Octacosane & $\mathrm{C}_{28} \mathrm{H}_{58}$ & $5279.4,4.217$ & 394.4533 & 286398 & \\
\hline $\begin{array}{l}\text { 3-tert-Butyl-1,2-dimethoxy-5- } \\
\text { methylbenzene }\end{array}$ & $\mathrm{C}_{13} \mathrm{H}_{20} \mathrm{O}_{2}$ ? & $5291.4,2.356$ & 208.1458 & 10237 & $9.21 \mathrm{E}-5$ \\
\hline $\begin{array}{l}\text { 1a,9b-dihydro- } \\
\text { cyclopropa[1]phenanthrene }\end{array}$ & $\mathrm{C}_{15} \mathrm{H}_{12}$ & $5327.41,2.201$ & 192.0934 & 14816 & \\
\hline $\begin{array}{l}\text { 2,3,4,5-Tetrahydro-6- } \\
\text { hexylpyridine }\end{array}$ & $\mathrm{C}_{11} \mathrm{H}_{21} \mathrm{~N}$ & $5339.42,3.827$ & 167.1669 & 107650 & \\
\hline 7-Phenylindene & $\mathrm{C}_{15} \mathrm{H}_{12}$ & $5357.42,2.189$ & 192.0934 & 15851 & \\
\hline $\begin{array}{l}\text { 3-Methyl-5-methylenenon-2- } \\
\text { en-4-ol }\end{array}$ & $\mathrm{C}_{11} \mathrm{H}_{20} \mathrm{O}$ ? & $5375.43,4.277$ & 168.1509 & 17946 & $1.61 \mathrm{E}-4$ \\
\hline $\begin{array}{l}\text { 2,2-Dimethyl-3,4- } \\
\text { undecanedione }\end{array}$ & $\mathrm{C}_{13} \mathrm{H}_{24} \mathrm{O}_{2} ?$ & $5381.43,4.285$ & 212.1771 & 61853 & $5.56 \mathrm{E}-4$ \\
\hline 3-Ethoxyacrylonitrile & $\mathrm{C}_{5} \mathrm{H}_{7} \mathrm{NO}$ & $5435.44,3.792$ & 97.0522 & 35367 & \\
\hline Tritriacontane & $\mathrm{C}_{33} \mathrm{H}_{68}$ & $5459.45,4.233$ & 464.5316 & 238170 & \\
\hline $\begin{array}{l}\text { 2-(2-Oxopropyl)- } \\
\text { cyclopentanone }\end{array}$ & $\mathrm{C}_{8} \mathrm{H}_{12} \mathrm{O}_{2}$ & $5477.46,3.891$ & 140.0832 & 38692 & \\
\hline Bicyclo[3.2.1] octan-1-ol & $\mathrm{C}_{8} \mathrm{H}_{14} \mathrm{O}$ & $5495.46,3.876$ & 126.1039 & 24579 & \\
\hline $\begin{array}{l}\text { 1-Hydroxy-2-methyl- } \\
\text { bicyclo[3.3.1]nonan-3-one }\end{array}$ & $\mathrm{C}_{10} \mathrm{H}_{16} \mathrm{O}_{2} ?$ & $5603.49,3.811$ & 168.1145 & 84771 & 7.62E-4 \\
\hline 2-Ethylanthracene & $\mathrm{C}_{16} \mathrm{H}_{14}$ & $5717.53,2.242$ & 206.109 & 12128 & \\
\hline 4,6-Dimethylundecane & $\mathrm{C}_{13} \mathrm{H}_{28}$ & $5729.53,4.222$ & 184.2186 & 105181 & \\
\hline Docosane & $\mathrm{C}_{22} \mathrm{H}_{46}$ & $5801.55,4.185$ & 310.3594 & 76783 & \\
\hline $\begin{array}{l}\text { 1,4-Bis(cyclohex-1-enyl)buta- } \\
\text { 1,3-diyne }\end{array}$ & $\mathrm{C}_{16} \mathrm{H}_{18}$ & $5867.57,4.264$ & 210.1403 & 42155 & \\
\hline 3,5-Diethylhex-5-ene-3-ol & $\mathrm{C}_{10} \mathrm{H}_{20} \mathrm{O}$ ? & $5945.59,4.211$ & 156.1509 & 53279 & 4.79E-4 \\
\hline $\begin{array}{l}\text { 6-Hexyl-4-oxa-5- } \\
\text { azaspiro[2.4]hept-5-ene }\end{array}$ & $\mathrm{C}_{11} \mathrm{H}_{19} \mathrm{NO}$ & $5975.6,3.756$ & 181.1461 & 72198 & \\
\hline 2,3-Octanedione & $\mathrm{C}_{8} \mathrm{H}_{14} \mathrm{O}_{2}$ & $5993.61,4.225$ & 142.0988 & 50333 & \\
\hline $\begin{array}{l}\text { 4-(1,1-Dimethylethyl)-2- } \\
\text { methyl-2-nitro-trans- } \\
\text { cyclohexanone }\end{array}$ & $\mathrm{C}_{11} \mathrm{H}_{19} \mathrm{NO}_{3}$ ? & $6203.67,4.268$ & 213.1359 & 28764 & $2.58 \mathrm{E}-4$ \\
\hline $\begin{array}{l}\text { (3R)-3-Phenyl-2,3-dihydro- } \\
\text { 1H-isoindol-1-one }\end{array}$ & $\mathrm{C}_{14} \mathrm{H}_{11} \mathrm{NO} ?$ & $6245.68,1.099$ & 209.0835 & 25254 & $2.27 \mathrm{E}-4$ \\
\hline 2-Methylhexadecane & $\mathrm{C}_{17} \mathrm{H}_{36}$ & $6377.72,4.284$ & 240.2812 & 244918 & \\
\hline 2-Naphthalenesulfonic acid & $\mathrm{C}_{10} \mathrm{H}_{8} \mathrm{O}_{3} \mathrm{~S} ?$ & $6545.76,1.097$ & 208.0189 & 403842 & $3.63 \mathrm{E}-3$ \\
\hline $\begin{array}{l}\text { cis-exo-7- } \\
\text { Oxabicyclo[2.2.1]hept-5-ene- } \\
\text { 2,3-diol }\end{array}$ & $\mathrm{C}_{6} \mathrm{H}_{8} \mathrm{O}_{3}$ ? & $6731.82,4.360$ & 128.0468 & 21755 & $1.95 \mathrm{E}-4$ \\
\hline 5-Carbamoyltetrazole & $\mathrm{C}_{2} \mathrm{H}_{3} \mathrm{~N}_{5} \mathrm{O}$ & $6887.86,4.643$ & 113.0332 & 7173 & \\
\hline
\end{tabular}




\begin{tabular}{|c|c|c|c|c|c|}
\hline Tetratetracontane & $\mathrm{C}_{44} \mathrm{H}_{90}$ & $6941.88,4.397$ & 618.7037 & 483099 & \\
\hline (S)-2-Ethylheptanol & $\mathrm{C}_{9} \mathrm{H}_{20} \mathrm{O}$ ? & $7049.91,4.590$ & 144.1509 & 58667 & $5.28 \mathrm{E}-4$ \\
\hline Benzene & $\mathrm{C}_{6} \mathrm{H}_{6}$ & $7446.02,1.090$ & 78.0464 & 64600 & \\
\hline $\begin{array}{l}\text { Diethyl 1,4-dihydro-2,6- } \\
\text { dimethyl-4-(3-pyridazinyl)-- } \\
\text { 3,5-pyridinedicarboxylate } \\
\end{array}$ & $\mathrm{C}_{17} \mathrm{H}_{21} \mathrm{~N}_{3} \mathrm{O}_{4}$ ? & $7650.08,1.728$ & 331.1527 & 10001 & $9.00 \mathrm{E}-5$ \\
\hline Pentatriacontane & $\mathrm{C}_{35} \mathrm{H}_{72}$ & $7854.14,5.422$ & 492.5629 & 178296 & \\
\hline $\begin{array}{l}\text { 2-(1,1-Dimethylprop-2-en-1- } \\
\text { yl)-1,3-dioxolane }\end{array}$ & $\mathrm{C}_{8} \mathrm{H}_{14} \mathrm{O}_{2}$ & $7902.15,1.163$ & 142.0988 & 34057 & \\
\hline (E)-Non-3-en-8-yn-2-one & $\mathrm{C}_{9} \mathrm{H}_{12} \mathrm{O}$ & $7926.16,1.379$ & 136.0883 & 7366 & \\
\hline $\begin{array}{l}\text { Ethyl (E)-4-Benzoyl-4- } \\
\text { (imidazolidin-2-ylidene)but-2- } \\
\text { enoate }\end{array}$ & $\mathrm{C}_{16} \mathrm{H}_{18} \mathrm{~N}_{2} \mathrm{O}_{3}$ ? & $7968.17,1.260$ & 286.1312 & 19868 & $1.78 \mathrm{E}-4$ \\
\hline $\begin{array}{l}\text { (R)-3-(4- } \\
\text { Methoxyphenyl)butanoic Acid }\end{array}$ & $\mathrm{C}_{11} \mathrm{H}_{14} \mathrm{O}_{3} ?$ & $7986.18,1.384$ & 194.0937 & 15139 & $1.36 \mathrm{E}-4$ \\
\hline $\begin{array}{l}\text { tert-Butyl(3- } \\
\text { Isopentyltetrahydrofuran-2- } \\
\text { ylidene)acetate }\end{array}$ & $\mathrm{C}_{15} \mathrm{H}_{26} \mathrm{O}_{3}$ ? & $7998.18,0.745$ & 254.1876 & 14007 & $1.26 \mathrm{E}-4$ \\
\hline $\begin{array}{l}\text { (1R,2R)-2,3-Dibenzyloxy-1-[2- } \\
\text { (1,3-dioxolan-2-yl)]ethylpropyl } \\
\text { methanesulfanate }\end{array}$ & $\mathrm{C}_{23} \mathrm{H}_{30} \mathrm{O}_{7} \mathrm{~S} ?$ & $7998.18,1.173$ & 450.1707 & 37050 & $3.334 \mathrm{E}-4$ \\
\hline $\begin{array}{l}\text { 2-(3,3-Dimethyl-1-butenyl)- } \\
\text { (E)-1-cyclohexene-1- } \\
\text { carboxaldehyde }\end{array}$ & $\mathrm{C}_{13} \mathrm{H}_{20} \mathrm{O}$ ? & $7998.18,1.493$ & 192.1509 & 6727 & $6.05 \mathrm{E}-5$ \\
\hline $\begin{array}{l}\text { 4-Ethoxycarbonyl-2,2,5- } \\
\text { trimethyl-3-(n-decyl)-2H- } \\
\text { pyrrole 1-oxide }\end{array}$ & $\mathrm{C}_{20} \mathrm{H}_{35} \mathrm{NO}_{3} ?$ & $8082.2,1.461$ & 337.2611 & 4285 & $3.85 \mathrm{E}-5$ \\
\hline $\begin{array}{l}\text { (S)-(E)-4-Acetoxy-1-phenyl-2- } \\
\text { dodecen-1-one }\end{array}$ & $\mathrm{C}_{20} \mathrm{H}_{28} \mathrm{O}_{3}$ ? & $8088.21,1.111$ & 316.2033 & 76200 & $6.85 \mathrm{E}-4$ \\
\hline Octanoic acid & $\mathrm{C}_{8} \mathrm{H}_{16} \mathrm{O}_{2}$ ? & $8130.22,1.441$ & 144.1145 & 4599 & 4.13E-5 \\
\hline $\begin{array}{l}\text { 2-Methoxy-,4- } \\
\text { (methoxycarbonyl)phenyl } \\
\text { benzoate }\end{array}$ & $\mathrm{C}_{16} \mathrm{H}_{14} \mathrm{O}_{5}$ ? & $8136.22,1.360$ & 286.0836 & 7711 & $6.93 \mathrm{E}-5$ \\
\hline $\begin{array}{l}\text { Methyl 5-(4-methoxyphenyl)- } \\
\text { 2,2,4,4-tetramethyl-3,5- } \\
\text { dioxopentanoate }\end{array}$ & $\mathrm{C}_{17} \mathrm{H}_{22} \mathrm{O}_{5}$ ? & $8166.23,1.377$ & 306.1462 & 14504 & $1.30 \mathrm{E}-4$ \\
\hline $\begin{array}{l}\text { 5-(Pyridine-4-yl)-1,3,4- } \\
\text { oxadiazole-2-(3H)-thione }\end{array}$ & $\mathrm{C}_{7} \mathrm{H}_{5} \mathrm{~N}_{3} \mathrm{OS}$ & $8208.24,1.205$ & 179.0148 & 32849 & \\
\hline $\begin{array}{l}\text { (3S)-3-Phenyl-2,3-dihydro-1H- } \\
\text { isoindol-1-one }\end{array}$ & $\mathrm{C}_{14} \mathrm{H}_{11} \mathrm{NO} ?$ & $8214.24,1.183$ & 209.0835 & 7174 & $6.45 \mathrm{E}-5$ \\
\hline
\end{tabular}

Table S15. Data obtained for the distillate fraction of 1 condensate-like oil by using GCxGC/(+EI) TOF MS. Data provided are based on ChromaTOF software prediction of the molecular ion $\mathrm{m} / \mathrm{z}$ value and the identification of the compound by using the EI mass spectral library match using a match factor threshold of 800 . Formulas with a question mark "?" might not have been accurately identified. The ratio of the peak area of each of these questionable compounds relative to all peak areas of the detected compounds is provided in the table below

\begin{tabular}{|l|c|c|c|c|}
\hline \multicolumn{1}{|c|}{ Name } & Formula & $\begin{array}{c}\text { Retention Times (s) on the } \\
\text { Primary and Secondary } \\
\text { Columns }\end{array}$ & $\begin{array}{c}\text { Molecular } \\
\text { Ion } \mathbf{m} / \mathbf{z}\end{array}$ & $\begin{array}{c}\text { Chromatographic } \\
\text { Peak Area }\end{array}$ \\
\hline 3-Methylheptane & $\mathrm{C}_{8} \mathrm{H}_{18}$ & $406.002,2.407$ & 114.1403 & 228664 \\
\hline
\end{tabular}




\begin{tabular}{|c|c|c|c|c|c|}
\hline $\begin{array}{l}\text { 1,1,3,4-Tetramethyl-cis- } \\
\text { cyclopentane }\end{array}$ & $\mathrm{C}_{9} \mathrm{H}_{18}$ & $406.002,2.438$ & 126.1403 & 115682 & \\
\hline 1,1,2-Trimethylcyclopentane & $\mathrm{C}_{8} \mathrm{H}_{16}$ & $424.007,2.309$ & 112.1247 & 266856 & \\
\hline Cyclopentane & $\mathrm{C}_{5} \mathrm{H}_{10}$ & $436.01,2.386$ & 70.0777 & 90859 & \\
\hline 1,3-Dimethyl-cis-cyclohexane & $\mathrm{C}_{8} \mathrm{H}_{16}$ & $454.016,2.391$ & 112.1247 & 2497862 & \\
\hline 4-Methyl-1-heptanol & $\mathrm{C}_{8} \mathrm{H}_{18} \mathrm{O}$ & $454.016,2.618$ & 130.1352 & 127008 & \\
\hline 5-Methyldodecane & $\mathrm{C}_{13} \mathrm{H}_{28}$ & $460.017,2.787$ & 184.2186 & 134169 & \\
\hline 2,4-Dimethylheptane & $\mathrm{C}_{9} \mathrm{H}_{20}$ & $466.019,2.839$ & 128.156 & 92074 & \\
\hline 1-Ethyl-2-methylcyclopentane & $\mathrm{C}_{8} \mathrm{H}_{16}$ & $472.021,2.469$ & 112.1247 & 183216 & \\
\hline 1,1-Dimethylcyclohexane & $\mathrm{C}_{8} \mathrm{H}_{16}$ & $478.022,2.433$ & 112.1247 & 371592 & \\
\hline 2,6-Dimethylheptane & $\mathrm{C}_{9} \mathrm{H}_{20}$ & $478.022,2.875$ & 128.156 & 688531 & \\
\hline Methyl Propargyl Ether & $\mathrm{C}_{4} \mathrm{H}_{6} \mathrm{O}$ & $490.026,2.671$ & 70.0413 & 40531 & \\
\hline $\begin{array}{l}\text { 1,2-Dimethyl-trans- } \\
\text { cyclohexane }\end{array}$ & $\mathrm{C}_{8} \mathrm{H}_{16}$ & $496.028,2.501$ & 112.1247 & 1044446 & \\
\hline $\begin{array}{l}\text { Dihydro-2H-pyran-2,6(3H)- } \\
\text { dione }\end{array}$ & $\mathrm{C}_{5} \mathrm{H}_{6} \mathrm{O}_{3}$ & $496.028,2.692$ & 114.0311 & 44648 & \\
\hline 2,5-Dimethylheptane & $\mathrm{C}_{9} \mathrm{H}_{20}$ & $496.028,2.926$ & 128.156 & 660487 & \\
\hline $\begin{array}{l}\text { (2S,1'S)-(-)-2-(1-Hydroxy-6- } \\
\text { methylheptyl)-2,5-dihydrofuran }\end{array}$ & $\mathrm{C}_{12} \mathrm{H}_{22} \mathrm{O}_{2}$ ? & $502.029,2.690$ & 198.1614 & 39612 & $4.81 \mathrm{E}-4$ \\
\hline 1,4-Dimethylcyclohexane & $\mathrm{C}_{8} \mathrm{H}_{16}$ & $514.033,2.541$ & 112.1247 & 582815 & \\
\hline (S)-(-)-Pipecolic acid & $\mathrm{C}_{6} \mathrm{H}_{11} \mathrm{NO}_{2}$ & $514.033,2.812$ & 129.0784 & 13027 & \\
\hline 3-Ethyl-2-methylhexane & $\mathrm{C}_{9} \mathrm{H}_{20}$ & $526.036,3.010$ & 128.156 & 38067 & \\
\hline 1,3,5-Trimethylcyclohexane & $\mathrm{C}_{9} \mathrm{H}_{18}$ & $538.04,2.793$ & 126.1403 & 307540 & \\
\hline 2,3-Dimethylheptane & $\mathrm{C}_{9} \mathrm{H}_{20}$ & $550.043,3.088$ & 128.156 & 192047 & \\
\hline 1,1,3-Trimethylcyclohexane & $\mathrm{C}_{9} \mathrm{H}_{18}$ & $556.045,2.818$ & 126.1403 & 2260261 & \\
\hline 6-Methylundecane & $\mathrm{C}_{12} \mathrm{H}_{26}$ & $580.052,3.204$ & 170.2029 & 1606124 & \\
\hline Ethylcyclohexane & $\mathrm{C}_{8} \mathrm{H}_{16}$ & $586.053,2.700$ & 112.1247 & 995042 & \\
\hline 1,2,4-Trimethylcyclohexane & $\mathrm{C}_{9} \mathrm{H}_{18}$ & $592.055,2.916$ & 126.1403 & 1013505 & \\
\hline $\begin{array}{l}\text { 4,5-Dimethyl-trans-1- } \\
\text { azabicyclo[3.2.0]heptane-3,7- } \\
\text { dione }\end{array}$ & $\mathrm{C}_{8} \mathrm{H}_{11} \mathrm{NO}_{2}$ & $592.055,3.244$ & 153.0784 & 65190 & \\
\hline Toluene & $\mathrm{C}_{7} \mathrm{H}_{8}$ & $598.057,2.062$ & 92.0621 & 150890 & \\
\hline 1,2,3-Trimethylcyclohexane & $\mathrm{C}_{9} \mathrm{H}_{18}$ & $616.062,2.957$ & 126.1403 & 11390 & \\
\hline 1-Ethyl-3-methylcyclopentane & $\mathrm{C}_{8} \mathrm{H}_{16}$ & $646.071,3.043$ & 112.1247 & 30743 & \\
\hline $\begin{array}{l}\text { 3-Butyl-3,5- } \\
\text { dimethylcyclohexanone }\end{array}$ & $\mathrm{C}_{12} \mathrm{H}_{22} \mathrm{O}$ ? & $652.072,3.214$ & 182.1665 & 172392 & $2.09 \mathrm{E}-3$ \\
\hline
\end{tabular}




\begin{tabular}{|c|c|c|c|c|c|}
\hline $\begin{array}{l}\text { 2,4-Bis(methylethyl)-2-methyl- } \\
\text { 2,5-dihydro-1H-imidazole }\end{array}$ & $\mathrm{C}_{10} \mathrm{H}_{20} \mathrm{~N}_{2} ?$ & $664.076,3.178$ & 168.1621 & 17236 & $2.09 \mathrm{E}-4$ \\
\hline 1,2,3-Trimethylcyclohexane & $\mathrm{C}_{9} \mathrm{H}_{18}$ & $670.078,3.029$ & 126.1403 & 610234 & \\
\hline 2,4-Dimethylhexane & $\mathrm{C}_{8} \mathrm{H}_{18}$ & $682.081,3.636$ & 114.1403 & 326024 & \\
\hline 1-Ethyl-4-methylcyclohexane & $\mathrm{C}_{9} \mathrm{H}_{18}$ & $706.088,3.106$ & 126.1403 & 1458057 & \\
\hline $\begin{array}{l}\text { N-(But-2-enoyl)prop-2- } \\
\text { enamide }\end{array}$ & $\mathrm{C}_{7} \mathrm{H}_{11} \mathrm{NO}$ & $706.088,3.355$ & 125.0835 & 27673 & \\
\hline Nonane & $\mathrm{C}_{9} \mathrm{H}_{20}$ & $706.088,3.682$ & 128.156 & 599857 & \\
\hline $\begin{array}{l}\text { 1,2-Diethyl-3- } \\
\text { methylcyclohexane }\end{array}$ & $\mathrm{C}_{11} \mathrm{H}_{22}$ & $718.091,3.333$ & 154.1716 & 293576 & \\
\hline Dodec-3-yn-1-ol & $\mathrm{C}_{12} \mathrm{H}_{22} \mathrm{O}$ ? & $736.096,3.470$ & 182.1665 & 26464 & $3.21 \mathrm{E}-4$ \\
\hline 2,6-Dimethyloctane & $\mathrm{C}_{10} \mathrm{H}_{22}$ & $736.096,3.739$ & 142.1716 & 1467384 & \\
\hline 6-Methylheptan-1-ol & $\mathrm{C}_{8} \mathrm{H}_{18} \mathrm{O}$ & $748.1,3.477$ & 130.1352 & 86756 & \\
\hline Octahydropentalene & $\mathrm{C}_{8} \mathrm{H}_{14}$ & $754.102,2.746$ & 110.109 & 180515 & \\
\hline 3-Ethyl-2-methylheptane & $\mathrm{C}_{10} \mathrm{H}_{22}$ & $772.107,3.759$ & 142.1716 & 1125217 & \\
\hline 3-Propylcyclohexene & $\mathrm{C}_{9} \mathrm{H}_{16}$ & $778.109,2.988$ & 124.1247 & 268884 & \\
\hline $\begin{array}{l}\text { 1-Ethyl-2-methyl-cis- } \\
\text { cyclohexane }\end{array}$ & $\mathrm{C}_{9} \mathrm{H}_{18}$ & $778.109,3.172$ & 126.1403 & 851447 & \\
\hline 1-Ethyl-1H-1,2,4-triazole & $\mathrm{C}_{4} \mathrm{H}_{7} \mathrm{~N}_{3}$ & $784.11,3.525$ & 97.0634 & 177174 & \\
\hline $\begin{array}{l}\text { 1-(Dodecycloxy)-4- } \\
\text { methoxybutan-2-one }\end{array}$ & $\mathrm{C}_{17} \mathrm{H}_{34} \mathrm{O}_{3}$ ? & $784.11,3.730$ & 286.2502 & 38996 & 4.73E-4 \\
\hline cis-3,4-Epoxy-1-cyclohexanol & $\mathrm{C}_{6} \mathrm{H}_{10} \mathrm{O}_{2} ?$ & $790.112,3.775$ & 114.0675 & 42625 & $5.17 \mathrm{E}-4$ \\
\hline 1-Ethyl-1-methylcyclohexane & $\mathrm{C}_{9} \mathrm{H}_{18}$ & $808.117,3.143$ & 126.1403 & 125110 & \\
\hline $\begin{array}{l}\text { 1-Methyl- } \\
\text { bicyclo[3.2.0]heptan-6-one }\end{array}$ & $\mathrm{C}_{8} \mathrm{H}_{12} \mathrm{O}$ & $814.119,2.726$ & 124.0883 & 20546 & \\
\hline 2,5,6-Trimethyldecane & $\mathrm{C}_{13} \mathrm{H}_{28}$ & $814.119,3.842$ & 184.2186 & 797364 & \\
\hline $\begin{array}{l}\text { 5-Acetyl-3-methyl-4,5- } \\
\text { dihydroisoxazole }\end{array}$ & $\mathrm{C}_{6} \mathrm{H}_{9} \mathrm{NO}_{2}$ & $826.122,3.818$ & 127.0628 & 110312 & \\
\hline 4-Methylnonane & $\mathrm{C}_{10} \mathrm{H}_{22}$ & $832.124,3.909$ & 142.1716 & 1515744 & \\
\hline $\begin{array}{l}\text { 1-Ethyl-cis-1,3-dimethyl- } \\
\text { cyclohexane }\end{array}$ & $\mathrm{C}_{10} \mathrm{H}_{20}$ & $838.126,3.507$ & 140.156 & 396622 & \\
\hline 2,2,4-Trimethyl-2H-imidazole & $\mathrm{C}_{6} \mathrm{H}_{10} \mathrm{~N}_{2}$ & $844.127,3.967$ & 110.0838 & 13757 & \\
\hline Methyl pentanoate & $\mathrm{C}_{6} \mathrm{H}_{12} \mathrm{O}_{2} ?$ & $844.127,4.170$ & 116.0832 & 48095 & $5.84 \mathrm{E}-4$ \\
\hline Propylcyclohexane & $\mathrm{C}_{9} \mathrm{H}_{18}$ & $856.131,3.284$ & 126.1403 & 585906 & \\
\hline 3-Methylnonane & $\mathrm{C}_{10} \mathrm{H}_{22}$ & $868.134,3.945$ & 142.1716 & 568746 & \\
\hline Octahydro-cis- $1 \mathrm{H}$-indene & $\mathrm{C}_{9} \mathrm{H}_{16}$ & $874.136,3.091$ & 124.1247 & 437469 & \\
\hline $\begin{array}{l}\text { (1R,2S)-1-Methylcyclohexene } \\
\text { oxide }\end{array}$ & $\mathrm{C}_{7} \mathrm{H}_{12} \mathrm{O}$ & $874.136,3.222$ & 112.0883 & 86213 & \\
\hline
\end{tabular}




\begin{tabular}{|c|c|c|c|c|c|}
\hline 2,3,3-Trimethyl-(Z)- 4-nonene & $\mathrm{C}_{12} \mathrm{H}_{24}$ & $874.136,3.525$ & 168.1873 & 129157 & \\
\hline Ethylbenzene & $\mathrm{C}_{8} \mathrm{H}_{10}$ & $886.14,2.372$ & 106.0777 & 56713 & \\
\hline 4,6-Dimethyldecan-1-ol & $\mathrm{C}_{12} \mathrm{H}_{26} \mathrm{O} ?$ & $898.143,3.513$ & 186.1978 & 191681 & $2.32 \mathrm{E}-3$ \\
\hline p-Xylene & $\mathrm{C}_{8} \mathrm{H}_{10}$ & $904.145,2.417$ & 106.0777 & 580482 & \\
\hline $\begin{array}{l}\text { 3-Cyclohexyl-(2S-trans)- } \\
\text { oxiranemethanol }\end{array}$ & $\mathrm{C}_{9} \mathrm{H}_{16} \mathrm{O}_{2} ?$ & $904.145,3.359$ & 156.1145 & 31656 & $3.84 \mathrm{E}-4$ \\
\hline $\begin{array}{l}\text { 1-Ethyl-1,3-dimethyl-trans- } \\
\text { cyclohexane }\end{array}$ & $\mathrm{C}_{10} \mathrm{H}_{20}$ & $922.15,3.574$ & 140.156 & 803911 & \\
\hline $\begin{array}{l}\text { 2,6,10,14- } \\
\text { Tetramethylhexadecane }\end{array}$ & $\mathrm{C}_{20} \mathrm{H}_{42}$ & $934.153,4.231$ & 282.3281 & 83769 & \\
\hline $\begin{array}{l}1,1,2,3- \\
\text { Tetramethylcyclohexane }\end{array}$ & $\mathrm{C}_{10} \mathrm{H}_{20}$ & $940.155,3.466$ & 140.156 & 780984 & \\
\hline 4-Methyleneheptane & $\mathrm{C}_{8} \mathrm{H}_{16}$ & $940.155,4.037$ & 112.1247 & 47788 & \\
\hline Octadecane & $\mathrm{C}_{18} \mathrm{H}_{38}$ & $952.158,4.262$ & 254.2968 & 24027 & \\
\hline $\begin{array}{l}\text { (2"E)-2'-Methyl-2'-(1",4",4"- } \\
\text { trimethylpent-2"- } \\
\text { enyloxy)propyl } \\
\text { cyclopropanecarboxylate }\end{array}$ & $\mathrm{C}_{16} \mathrm{H}_{28} \mathrm{O}_{3}$ & $958.16,3.503$ & 268.2033 & 75733 & \\
\hline Tetrahydro-3,3'-bifuran & $\mathrm{C}_{8} \mathrm{H}_{10} \mathrm{O}_{2}$ ? & $958.16,4.016$ & 138.0675 & 31790 & $3.86 \mathrm{E}-4$ \\
\hline 1-(1-Propynyl)cyclopropanol & $\mathrm{C}_{6} \mathrm{H}_{8} \mathrm{O}$ ? & $964.162,3.417$ & 96.057 & 47951 & $5.82 \mathrm{E}-4$ \\
\hline Decane & $\mathrm{C}_{10} \mathrm{H}_{22}$ & $976.165,4.063$ & 142.1716 & 2505247 & \\
\hline $\begin{array}{l}\text { 1-Isopropyl-4- } \\
\text { methylcyclohexane }\end{array}$ & $\mathrm{C}_{10} \mathrm{H}_{20}$ & $988.169,3.596$ & 140.156 & 407611 & \\
\hline $\begin{array}{l}\text { 2,4-Bis(dimethylethyl)-2- } \\
\text { methyl-2,5-dihydro-1H- } \\
\text { imidazole }\end{array}$ & $\mathrm{C}_{12} \mathrm{H}_{24} \mathrm{~N}_{2}$ & $988.169,3.814$ & 196.1934 & 25961 & \\
\hline Undecane & $\mathrm{C}_{11} \mathrm{H}_{24}$ & $994.171,4.300$ & 156.1873 & 170967 & \\
\hline $\begin{array}{l}\text { 3-Methoxymethylhept-1-yn-3- } \\
\text { ol }\end{array}$ & $\mathrm{C}_{9} \mathrm{H}_{16} \mathrm{O}_{2}$ & $1006.17,3.577$ & 156.1145 & 32143 & \\
\hline $\begin{array}{l}\text { 1-(1-Methylcyclohexyl)- } \\
\text { ethanone }\end{array}$ & $\mathrm{C}_{9} \mathrm{H}_{16} \mathrm{O}$ & $1006.17,3.662$ & 140.1196 & 808183 & \\
\hline 6-Methyl-2-hepten-4-one & $\mathrm{C}_{8} \mathrm{H}_{14} \mathrm{O}$ & $1012.18,3.595$ & 126.1039 & 396396 & \\
\hline 1,2-Dimethylbenzene & $\mathrm{C}_{8} \mathrm{H}_{10}$ & $1024.18,2.427$ & 106.0777 & 312208 & \\
\hline $\begin{array}{l}\text { 2-Methyl-2-(1-methyl-1- } \\
\text { propenyl)cyclobutanone }\end{array}$ & $\mathrm{C}_{9} \mathrm{H}_{14} \mathrm{O}$ & $1024.18,3.050$ & 138.1039 & 10111 & \\
\hline 2,6-Dimethylnonane & $\mathrm{C}_{11} \mathrm{H}_{24}$ & $1024.18,4.351$ & 156.1873 & 1441833 & \\
\hline 2-Methyl-4-hexyn-3-ol & $\mathrm{C}_{7} \mathrm{H}_{12} \mathrm{O}$ & $1030.18,3.997$ & 112.0883 & 26630 & \\
\hline $\begin{array}{l}\text { 4-Acetoxy-2,5- } \\
\text { divinyltetrahydrofuran-3-yl } \\
\text { but-3-enoate }\end{array}$ & $\mathrm{C}_{14} \mathrm{H}_{18} \mathrm{O}_{5}$ ? & $1042.18,3.991$ & 266.1149 & 13219 & $1.60 \mathrm{E}-4$ \\
\hline 1-Pentadecanol & $\mathrm{C}_{15} \mathrm{H}_{32} \mathrm{O}$ ? & $1048.19,3.927$ & 228.2448 & 18138 & $2.20 \mathrm{E}-4$ \\
\hline $\begin{array}{l}\text { 4,8-Dimethyl-3,4-epoxy-7- } \\
\text { nonen-2-ol }\end{array}$ & $\mathrm{C}_{11} \mathrm{H}_{20} \mathrm{O}_{2}$ ? & $1054.19,3.937$ & 184.1458 & 10142 & $1.23 \mathrm{E}-4$ \\
\hline $\begin{array}{l}\text { cis-9-Oxabicyclo[6.1.0]non-2- } \\
\text { ene }\end{array}$ & $\mathrm{C}_{8} \mathrm{H}_{12} \mathrm{O}$ & $1066.19,3.408$ & 124.0883 & 11250 & \\
\hline
\end{tabular}




\begin{tabular}{|c|c|c|c|c|c|}
\hline Ethylpropylcyclopentane & $\mathrm{C}_{10} \mathrm{H}_{20}$ & $1072.19,3.662$ & 140.156 & 333873 & \\
\hline 3-Methyldodecane & $\mathrm{C}_{13} \mathrm{H}_{28}$ & $1078.19,4.350$ & 184.2186 & 128090 & \\
\hline 2,3,3-Trimethylcyclobutanone & $\mathrm{C}_{7} \mathrm{H}_{12} \mathrm{O}$ & $1084.2,4.061$ & 112.0883 & 25470 & \\
\hline Decan-1-ol & $\mathrm{C}_{10} \mathrm{H}_{22} \mathrm{O}$ ? & $1090.2,4.029$ & 158.1665 & 67430 & $8.19 \mathrm{E}-4$ \\
\hline $\begin{array}{l}\text { 2,2-Dimethyl-3-(3-oxobutyl)-, } \\
\text { methyl (1R-cis)- } \\
\text { cyclopropanecarboxylate }\end{array}$ & $\mathrm{C}_{11} \mathrm{H}_{18} \mathrm{O}_{3} ?$ & $1096.2,3.795$ & 198.125 & 47221 & $5.73 \mathrm{E}-4$ \\
\hline (1-Methylethyl)benzene & $\mathrm{C}_{9} \mathrm{H}_{12}$ & $1102.2,2.580$ & 120.0934 & 17659 & \\
\hline 2-But-1-enyltetrahydrofuran & $\mathrm{C}_{8} \mathrm{H}_{14} \mathrm{O}$ & $1102.2,4.036$ & 126.1039 & 26437 & \\
\hline 3,7-Dimethylnonane & $\mathrm{C}_{11} \mathrm{H}_{24}$ & $1102.2,4.346$ & 156.1873 & 474077 & \\
\hline 2,5-Dimethylhex-4-enoic acid & $\mathrm{C}_{8} \mathrm{H}_{14} \mathrm{O}_{2}$ ? & $1108.2,3.949$ & 142.0988 & 13313 & $1.61 \mathrm{E}-4$ \\
\hline 3-Methyl-1-hexen-5-one & $\mathrm{C}_{7} \mathrm{H}_{12} \mathrm{O}$ & $1120.21,4.096$ & 112.0883 & 82107 & \\
\hline 2,2-Dimethylheptanenitrile & $\mathrm{C}_{9} \mathrm{H}_{17} \mathrm{~N}$ & $1132.21,3.926$ & 139.1356 & 83236 & \\
\hline $\begin{array}{l}\text { N-(1-Methylpropylidene)- 2- } \\
\text { butanamine }\end{array}$ & $\mathrm{C}_{8} \mathrm{H}_{17} \mathrm{~N}$ & $1132.21,4.061$ & 127.1356 & 26293 & \\
\hline Methyl nonanoate & $\mathrm{C}_{10} \mathrm{H}_{20} \mathrm{O}_{2}$ ? & $1132.21,4.105$ & 172.1458 & 26486 & $3.21 \mathrm{E}-4$ \\
\hline $\begin{array}{l}\text { (+-)-cis-3-Hydroxymethyl-2,2- } \\
\text { dimethyl-1-cyclobutaneacetic } \\
\text { acid }\end{array}$ & $\mathrm{C}_{9} \mathrm{H}_{16} \mathrm{O}_{3}$ ? & $1138.21,3.904$ & 172.1094 & 55047 & $6.68 \mathrm{E}-4$ \\
\hline $\begin{array}{l}\text { 1,1-Dimethyl-2-oxopropyl- } \\
\text { N,N-pentamethylenecarbamate }\end{array}$ & $\mathrm{C}_{11} \mathrm{H}_{19} \mathrm{NO}_{3}$ ? & $1138.21,4.094$ & 213.1359 & 8844 & $1.07 \mathrm{E}-4$ \\
\hline 3-Decyn-1-ol & $\mathrm{C}_{10} \mathrm{H}_{18} \mathrm{O}$ ? & $1156.22,4.123$ & 154.1352 & 56324 & $6.84 \mathrm{E}-4$ \\
\hline 1,3,5-Triazine-2,4-diamine & $\mathrm{C}_{3} \mathrm{H}_{5} \mathrm{~N}_{5}$ & $1162.22,3.535$ & 111.0539 & 24088 & \\
\hline (S)-Undec-1-en-4-ol & $\mathrm{C}_{11} \mathrm{H}_{22} \mathrm{O}$ ? & $1162.22,3.591$ & 170.1665 & 49397 & $6.00 \mathrm{E}-4$ \\
\hline $\begin{array}{l}\text { 1,1-Dimethyl-3-methylene- } \\
\text { cyclopentane }\end{array}$ & $\mathrm{C}_{8} \mathrm{H}_{14}$ & $1168.22,3.782$ & 110.109 & 62665 & \\
\hline $\begin{array}{l}\text { trans-1-Formyl-2-(1- } \\
\text { methylethenyl)cyclopropane }\end{array}$ & $\mathrm{C}_{7} \mathrm{H}_{10} \mathrm{O}$ & $1174.22,3.782$ & 110.0726 & 97221 & \\
\hline 5-Methyldecane & $\mathrm{C}_{11} \mathrm{H}_{24}$ & $1174.22,4.402$ & 156.1873 & 482015 & \\
\hline 4-Methyl-5-propylnonane & $\mathrm{C}_{13} \mathrm{H}_{28}$ & $1192.23,4.392$ & 184.2186 & 314314 & \\
\hline Dioctyldisulfide & $\mathrm{C}_{16} \mathrm{H}_{34} \mathrm{~S}_{2}$ & $1192.23,4.718$ & 290.2096 & 25577 & \\
\hline 2-Methyldecane & $\mathrm{C}_{11} \mathrm{H}_{24}$ & $1198.23,4.433$ & 156.1873 & 381298 & \\
\hline Phenylacetaldehyde & $\mathrm{C}_{8} \mathrm{H}_{8} \mathrm{O}$ & $1222.24,2.634$ & 120.057 & 45787 & \\
\hline (1-Methylpropyl)cyclohexane & $\mathrm{C}_{10} \mathrm{H}_{20}$ & $1222.24,3.629$ & 140.156 & 39982 & \\
\hline Octacosane & $\mathrm{C}_{28} \mathrm{H}_{58}$ & $1222.24,4.378$ & 394.4533 & 64166 & \\
\hline Butylcyclohexane & $\mathrm{C}_{10} \mathrm{H}_{20}$ & $1234.24,3.687$ & 140.156 & 473311 & \\
\hline
\end{tabular}




\begin{tabular}{|c|c|c|c|c|c|}
\hline 3-Methyldecane & $\mathrm{C}_{11} \mathrm{H}_{24}$ & $1234.24,4.413$ & 156.1873 & 331679 & \\
\hline cis-1,2-Diethylcyclohexane & $\mathrm{C}_{10} \mathrm{H}_{20}$ & $1240.24,3.955$ & 140.156 & 395307 & \\
\hline 1-Ethyl-2-methylbenzene & $\mathrm{C}_{9} \mathrm{H}_{12}$ & $1264.25,2.633$ & 120.0934 & 256545 & \\
\hline 7-Methyl-(Z)-5-undecene & $\mathrm{C}_{12} \mathrm{H}_{24}$ & $1264.25,3.855$ & 168.1873 & 109336 & \\
\hline Ethylcyclooctane & $\mathrm{C}_{10} \mathrm{H}_{20}$ & $1276.25,3.965$ & 140.156 & 330810 & \\
\hline 1,2,3-Trimethylbenzene & $\mathrm{C}_{9} \mathrm{H}_{12}$ & $1288.25,2.654$ & 120.0934 & 607085 & \\
\hline 3-Ethoxy-1-propyne & $\mathrm{C}_{5} \mathrm{H}_{8} \mathrm{O}$ & $1294.26,3.875$ & 84.057 & 34761 & \\
\hline 1,1,2-Trimethylcyclohexane & $\mathrm{C}_{9} \mathrm{H}_{18}$ & $1300.26,4.015$ & 126.1403 & 45501 & \\
\hline S,S-Dioxide-2-methylthiolane & $\mathrm{C}_{5} \mathrm{H}_{10} \mathrm{O}_{2} \mathrm{~S} ?$ & $1300.26,4.343$ & 134.0396 & 12954 & $1.57 \mathrm{E}-4$ \\
\hline $\begin{array}{l}\text { (Z/E)-2,2,4,5-Tetramethyl-3- } \\
\text { heptene }\end{array}$ & $\mathrm{C}_{11} \mathrm{H}_{22}$ & $1312.26,4.283$ & 154.1716 & 29598 & \\
\hline $\begin{array}{l}\text { 3,3-Dimethylcyclohex-1-en-1- } \\
\text { yl but-2-enoate }\end{array}$ & $\mathrm{C}_{12} \mathrm{H}_{18} \mathrm{O}_{2} ?$ & $1324.27,3.885$ & 194.1301 & 56305 & $6.83 \mathrm{E}-4$ \\
\hline $\begin{array}{l}\text { 2-hydroxylactone } \\
\text { cyclohexaneacetic acid }\end{array}$ & $\mathrm{C}_{8} \mathrm{H}_{10} \mathrm{O}_{2}$ ? & $1324.27,4.101$ & 138.0675 & 21075 & $2.56 \mathrm{E}-4$ \\
\hline 4-Hydroxy- D-isovaline & $\mathrm{C}_{5} \mathrm{H}_{11} \mathrm{NO}_{3}$ & $1324.27,4.651$ & 133.0733 & 28167 & \\
\hline 3,3-Dimethylpentane & $\mathrm{C}_{7} \mathrm{H}_{16}$ & $1330.27,4.636$ & 100.1247 & 33786 & \\
\hline Pentadecane & $\mathrm{C}_{15} \mathrm{H}_{32}$ & $1336.27,4.620$ & 212.2499 & 27105 & \\
\hline $\begin{array}{l}3^{\prime}, 3^{\prime}, 5^{\prime}-\text { Trimethylcyclohexyl } \\
\text { (cis)-(3- } \\
\text { thienylglyoxyl)carboxylate }\end{array}$ & $\mathrm{C}_{15} \mathrm{H}_{20} \mathrm{O}_{3} \mathrm{~S}$ ? & $1348.27,3.907$ & 280.1128 & 78381 & $9.52 \mathrm{E}-4$ \\
\hline Cyclohexanone & $\mathrm{C}_{6} \mathrm{H}_{10} \mathrm{O}$ & $1348.27,4.387$ & 98.0726 & 15783 & \\
\hline Dodecane & $\mathrm{C}_{12} \mathrm{H}_{26}$ & $1366.28,4.407$ & 170.2029 & 3424632 & \\
\hline 1-Ethyl-4-methylbenzene & $\mathrm{C}_{9} \mathrm{H}_{12}$ & $1372.28,2.602$ & 120.0934 & 47914 & \\
\hline $\begin{array}{l}\text { 1,1-Dimethyl-2-propyl- } \\
\text { cyclohexane }\end{array}$ & $\mathrm{C}_{11} \mathrm{H}_{22}$ & $1372.28,3.795$ & 154.1716 & 468898 & \\
\hline $\begin{array}{l}\text { 2,3,4,5-Tetrahydro-6-propyl- } \\
\text { pyridine }\end{array}$ & $\mathrm{C}_{8} \mathrm{H}_{15} \mathrm{~N}$ & $1384.28,3.902$ & 125.1199 & 49669 & \\
\hline $\begin{array}{l}\text { 1-Hexyl-3-methyl- } \\
\text { cyclopentane }\end{array}$ & $\mathrm{C}_{12} \mathrm{H}_{24}$ & $1390.28,4.049$ & 168.1873 & 143076 & \\
\hline Tridecane & $\mathrm{C}_{13} \mathrm{H}_{28}$ & $1390.28,4.695$ & 184.2186 & 642683 & \\
\hline $\begin{array}{l}\text { (4RS,5S)-5-Methylhept-2-yn-4- } \\
\text { ol }\end{array}$ & $\mathrm{C}_{8} \mathrm{H}_{14} \mathrm{O}$ & $1396.29,3.897$ & 126.1039 & 86182 & \\
\hline $\begin{array}{l}\text { 1-Ethyl-4-methyl-trans- } \\
\text { cyclohexane }\end{array}$ & $\mathrm{C}_{9} \mathrm{H}_{18}$ & $1402.29,3.975$ & 126.1403 & 485500 & \\
\hline $\begin{array}{l}\text { 1-Hydroxymethyl-1,3- } \\
\text { dimethylcyclopentane }\end{array}$ & $\mathrm{C}_{8} \mathrm{H}_{16} \mathrm{O}$ & $1414.29,4.003$ & 128.1196 & 214240 & \\
\hline 2,6,10-trimethyltridecane & $\mathrm{C}_{16} \mathrm{H}_{34}$ & $1420.29,4.673$ & 226.2655 & 146084 & \\
\hline 3,7-Dimethyldecane & $\mathrm{C}_{12} \mathrm{H}_{26}$ & $1444.3,4.654$ & 170.2029 & 470598 & \\
\hline
\end{tabular}




\begin{tabular}{|c|c|c|c|c|c|}
\hline $\begin{array}{l}(3 \mathrm{R}, 3 \mathrm{aR}, 6 \mathrm{R}, 7 \mathrm{aR}) \text {-Perhydro- } \\
\text { 3,6-dimethyl-2- } \\
\text { benzo[b]furanone }\end{array}$ & $\mathrm{C}_{10} \mathrm{H}_{16} \mathrm{O}_{2} ?$ & $1450.3,3.770$ & 168.1145 & 12119 & $1.47 \mathrm{E}-4$ \\
\hline (Z)-6-Undecen-3-ol & $\mathrm{C}_{11} \mathrm{H}_{22} \mathrm{O}$ ? & $1456.3,3.727$ & 170.1665 & 17928 & $2.17 \mathrm{E}-4$ \\
\hline 2,4-Dimethyl-1,3-hexanediol & $\mathrm{C}_{8} \mathrm{H}_{18} \mathrm{O}_{2}$ ? & $1456.3,4.284$ & 146.1301 & 32396 & 3.93E-4 \\
\hline Decahydro-trans-naphthalene & $\mathrm{C}_{10} \mathrm{H}_{18}$ & $1468.31,3.338$ & 138.1403 & 287771 & \\
\hline Methyl(1-methylethyl)benzene & $\mathrm{C}_{10} \mathrm{H}_{14}$ & $1492.31,2.767$ & 134.109 & 77324 & \\
\hline $\begin{array}{l}\text { 1-Isopropyl-1-methyl- } \\
\text { cyclohexane }\end{array}$ & $\mathrm{C}_{10} \mathrm{H}_{20}$ & $1492.31,3.899$ & 140.156 & 39596 & \\
\hline 1-Methoxy-2-butyne & $\mathrm{C}_{5} \mathrm{H}_{8} \mathrm{O}$ & $1492.31,4.266$ & 84.057 & 17356 & \\
\hline $\begin{array}{l}\text { (1R,3S)-7,7-Dimethyl-2- } \\
\text { oxabicyclo[4.1.1.]octan-3-one }\end{array}$ & $\mathrm{C}_{9} \mathrm{H}_{14} \mathrm{O}_{2} ?$ & $1504.32,3.862$ & 154.0988 & 60484 & 7.34E-4 \\
\hline $\begin{array}{l}\text { 5-Hydroxymethyl-2- } \\
\text { tetrahydrofuraldehyde-3-one }\end{array}$ & $\mathrm{C}_{6} \mathrm{H}_{8} \mathrm{O}_{4}$ & $1510.32,4.584$ & 144.0417 & 41458 & \\
\hline 2-(1-Phenylethyl)phenol & $\mathrm{C}_{14} \mathrm{H}_{14} \mathrm{O}$ & $1528.32,4.594$ & 198.1039 & 113696 & \\
\hline 1,1-Dimethoxyprop-2-yne & $\mathrm{C}_{5} \mathrm{H}_{8} \mathrm{O}_{2}$ & $1552.33,4.124$ & 100.0519 & 45060 & \\
\hline 6-Methylundecane & $\mathrm{C}_{12} \mathrm{H}_{26}$ & $1564.33,4.629$ & 170.2029 & 880101 & \\
\hline Methylcycloheptane & $\mathrm{C}_{8} \mathrm{H}_{16}$ & $1576.34,4.315$ & 112.1247 & 56919 & \\
\hline $\begin{array}{l}\text { 2,2,5-Trimethyl-, (S)-6-hepten- } \\
\text { 1-ol }\end{array}$ & $\mathrm{C}_{10} \mathrm{H}_{20} \mathrm{O} ?$ & $1582.34,4.153$ & 156.1509 & 42516 & $5.16 \mathrm{E}-4$ \\
\hline 2-Acetylmethylnorbornane & $\mathrm{C}_{10} \mathrm{H}_{16} \mathrm{O}$ & $1588.34,3.561$ & 152.1196 & 9884 & \\
\hline 5-Ethyl-5-methyl-2-oxepanone & $\mathrm{C}_{9} \mathrm{H}_{16} \mathrm{O}_{2}$ & $1588.34,4.139$ & 156.1145 & 37345 & \\
\hline 5-Butylnonane & $\mathrm{C}_{13} \mathrm{H}_{28}$ & $1588.34,4.623$ & 184.2186 & 254682 & \\
\hline $\begin{array}{l}4,7- \\
\text { Dimethylhexahydrocyclopenta } \\
\text { pyran-3-one }\end{array}$ & $\mathrm{C}_{10} \mathrm{H}_{16} \mathrm{O}_{2}$ & $1606.35,3.982$ & 168.1145 & 21639 & \\
\hline Tetradecane & $\mathrm{C}_{14} \mathrm{H}_{30}$ & $1612.35,4.906$ & 198.2342 & 77183 & \\
\hline Aminoacetonitrile & $\mathrm{C}_{2} \mathrm{H}_{4} \mathrm{~N}_{2}$ & $1618.35,4.265$ & 56.0369 & 15303 & \\
\hline 1-Methyl-2-propylbenzene & $\mathrm{C}_{10} \mathrm{H}_{14}$ & $1624.35,2.793$ & 134.109 & 156612 & \\
\hline $\begin{array}{l}\text { 3,6-Dimethylhexahydro-1- } \\
\text { benzofuran-2(3H)-one }\end{array}$ & $\mathrm{C}_{10} \mathrm{H}_{16} \mathrm{O}_{2}$ & $1624.35,3.564$ & 168.1145 & 81045 & \\
\hline 2,6,10-Trimethyltridecane & $\mathrm{C}_{16} \mathrm{H}_{34}$ & $1630.35,4.550$ & 226.2655 & 52368 & \\
\hline $\begin{array}{l}\text { (2S)-2-(2- } \\
\text { Tetrahydropyranyloxy)propana } \\
1\end{array}$ & $\mathrm{C}_{8} \mathrm{H}_{14} \mathrm{O}_{3}$ & $1630.35,4.834$ & 158.0937 & 11561 & \\
\hline $\begin{array}{l}\text { 3-Methylene-15- } \\
\text { methoxypentadecanol }\end{array}$ & $\mathrm{C}_{18} \mathrm{H}_{36} \mathrm{O}_{3} ?$ & $1636.35,3.912$ & 300.2659 & 11582 & $1.40 \mathrm{E}-4$ \\
\hline Butylbenzene & $\mathrm{C}_{10} \mathrm{H}_{14}$ & $1642.36,2.805$ & 134.109 & 14147 & \\
\hline 3-Methylundecane & $\mathrm{C}_{12} \mathrm{H}_{26}$ & $1642.36,4.593$ & 170.2029 & 376205 & \\
\hline 2-Ethylbutyronitrile & $\mathrm{C}_{6} \mathrm{H}_{11} \mathrm{~N}$ & $1648.36,3.901$ & 97.0886 & 20942 & \\
\hline
\end{tabular}




\begin{tabular}{|c|c|c|c|c|c|}
\hline Pentylcyclohexane & $\mathrm{C}_{11} \mathrm{H}_{22}$ & $1654.36,3.888$ & 154.1716 & 403617 & \\
\hline 1-(2-Hydroxyethoxy)tridecane & $\mathrm{C}_{15} \mathrm{H}_{32} \mathrm{O}_{2}$ ? & $1654.36,4.856$ & 244.2397 & 10083 & $1.22 \mathrm{E}-4$ \\
\hline $\begin{array}{l}\text { Decahydro-2-methyl- } \\
\text { naphthalene }\end{array}$ & $\mathrm{C}_{11} \mathrm{H}_{20}$ & $1660.36,3.543$ & 152.156 & 357694 & \\
\hline $\begin{array}{l}\text { (cis)-1-Butyl-2- } \\
\text { hexylcyclopropane }\end{array}$ & $\mathrm{C}_{13} \mathrm{H}_{26}$ & $1666.36,4.140$ & 182.2029 & 51292 & \\
\hline 4-Ethyl-1,2-dimethylbenzene & $\mathrm{C}_{10} \mathrm{H}_{14}$ & $1672.37,2.757$ & 134.109 & 280936 & \\
\hline 10-Acetoxydecanal & $\mathrm{C}_{12} \mathrm{H}_{22} \mathrm{O}_{3}$ ? & $1690.37,4.161$ & 214.1563 & 309050 & $3.75 \mathrm{E}-3$ \\
\hline $\begin{array}{l}\text { (1R,5S,6S)-5-Methyl-6- } \\
\text { carbethoxy-2- } \\
\text { oxabicyclo[3.1.0]hexane }\end{array}$ & $\mathrm{C}_{9} \mathrm{H}_{14} \mathrm{O}_{3}$ ? & $1702.37,4.089$ & 170.0937 & 19545 & 2.37E-4 \\
\hline (1-Methylpropyl)benzene & $\mathrm{C}_{10} \mathrm{H}_{14}$ & $1714.38,2.772$ & 134.109 & 47405 & \\
\hline $\begin{array}{l}\text { (Z)-3-(Hydroxymethyl)-7- } \\
\text { methylocta-2,6-dien-1-yl } \\
\text { acetate }\end{array}$ & $\mathrm{C}_{12} \mathrm{H}_{20} \mathrm{O}_{3}$ ? & $1720.38,4.002$ & 212.1407 & 82626 & $1.00 \mathrm{E}-3$ \\
\hline $\begin{array}{l}\text { E-2-Methyl-3- } \\
\text { (phenylsulfonyl)undec-4-ene }\end{array}$ & $\mathrm{C}_{18} \mathrm{H}_{28} \mathrm{O}_{2} \mathrm{~S}$ ? & $1720.38,4.417$ & 308.1805 & 28713 & $3.48 \mathrm{E}-4$ \\
\hline 3,3,6-Trimethyl-1,2-dioxane & $\mathrm{C}_{7} \mathrm{H}_{13} \mathrm{O}_{2}$ & $1726.38,4.545$ & 129.091 & 29348 & \\
\hline 1,17-Octadecadiene & $\mathrm{C}_{18} \mathrm{H}_{34}$ & $1738.38,4.126$ & 250.2655 & 29165 & \\
\hline $\begin{array}{l}\text { 2,4,9,11-Tetramethyldodeca- } \\
\text { 5,7-diyne-4,9-diol }\end{array}$ & $\mathrm{C}_{16} \mathrm{H}_{26} \mathrm{O}_{2}$ ? & $1744.39,4.783$ & 250.1927 & 26964 & $3.27 \mathrm{E}-4$ \\
\hline 1-Methyldecahydronaphthalene & $\mathrm{C}_{11} \mathrm{H}_{20}$ & $1756.39,3.487$ & 152.156 & 320803 & \\
\hline 1-Ethyl-2,4-dimethylbenzene & $\mathrm{C}_{10} \mathrm{H}_{14}$ & $1768.39,2.751$ & 134.109 & 52985 & \\
\hline $\begin{array}{l}\text { 1-Methyl-2-(1-methylethyl)- } \\
\text { benzene }\end{array}$ & $\mathrm{C}_{10} \mathrm{H}_{14}$ & $1780.4,2.746$ & 134.109 & 134573 & \\
\hline $\begin{array}{l}\text { 1- } \\
\text { (Hydroxycarbonyl)cyclohexane }\end{array}$ & $\mathrm{C}_{7} \mathrm{H}_{12} \mathrm{O}_{2}$ & $1786.4,4.003$ & 128.0832 & 34268 & \\
\hline 2,6-Dimethylundecane & $\mathrm{C}_{13} \mathrm{H}_{28}$ & $1786.4,4.809$ & 184.2186 & 2724625 & \\
\hline 4-Methylphenyl acetone & $\mathrm{C}_{10} \mathrm{H}_{12} \mathrm{O}$ & $1792.4,2.952$ & 148.0883 & 66356 & \\
\hline $\begin{array}{l}\text { anti-2- } \\
\text { Propylbicyclo[3.1.0]hexane- } \\
\text { 1,2,8-triene }\end{array}$ & $\mathrm{C}_{9} \mathrm{H}_{16}$ & $1804.4,3.856$ & 124.1247 & 23267 & \\
\hline 2-(4'-Methylphenyl)propanal & $\mathrm{C}_{10} \mathrm{H}_{12} \mathrm{O}$ ? & $1822.41,2.903$ & 148.0883 & 57290 & $6.95 \mathrm{E}-4$ \\
\hline $\begin{array}{l}\text { 1-Methyl-2- } \\
\text { (tricyclo[4.1.0.0(2,7)]hept-1- } \\
\text { yl)benzene }\end{array}$ & $\mathrm{C}_{14} \mathrm{H}_{16}$ & $1822.41,2.951$ & 184.1247 & 20060 & \\
\hline 1-Methyl-2-pentylcyclohexane & $\mathrm{C}_{12} \mathrm{H}_{24}$ & $1822.41,4.104$ & 168.1873 & 308628 & \\
\hline $\begin{array}{l}\text { 3-Hydroxy-2,2-dimethyl-1- } \\
\text { phenyl-1-butanone }\end{array}$ & $\mathrm{C}_{12} \mathrm{H}_{16} \mathrm{O}_{2}$ ? & $1828.41,2.935$ & 192.1145 & 16484 & $2.00 \mathrm{E}-4$ \\
\hline $\begin{array}{l}\text { (3,5-cis)-3-Pentyl-5-methyl- } \\
\text { 1,2-oxathiolane-(2,3-trans)-2- } \\
\text { oxide }\end{array}$ & $\mathrm{C}_{9} \mathrm{H}_{18} \mathrm{O}_{2} \mathrm{~S}$ ? & $1834.41,4.039$ & 190.1022 & 46389 & $5.63 \mathrm{E}-4$ \\
\hline $\begin{array}{l}\text { 1,2,3,6,7,7a-Hexahydro-1-(2- } \\
\text { hydroxy-1-methylethyl)-, 7,3a- } \\
\text { (epoxymethano)inden-9-one }\end{array}$ & $\mathrm{C}_{13} \mathrm{H}_{18} \mathrm{O}_{3}$ ? & $1852.42,2.924$ & 222.125 & 23993 & $2.91 \mathrm{E}-4$ \\
\hline 2-Ethyldodecane & $\mathrm{C}_{13} \mathrm{H}_{28}$ ? & $1864.42,4.721$ & 184.2186 & 73201 & \\
\hline
\end{tabular}




\begin{tabular}{|c|c|c|c|c|c|}
\hline Nopyldiformamide & $\mathrm{C}_{13} \mathrm{H}_{19} \mathrm{NO}_{2}$ ? & $1882.43,2.900$ & 221.141 & 13601 & $1.65 \mathrm{E}-4$ \\
\hline 3,4-Dimethylcumene & $\mathrm{C}_{11} \mathrm{H}_{16}$ & $1888.43,2.853$ & 148.1247 & 40741 & \\
\hline 3-Ethynyl-2,2-dimethyloxirane & $\mathrm{C}_{6} \mathrm{H}_{8} \mathrm{O}$ & $1906.43,3.391$ & 96.057 & 10776 & \\
\hline $\begin{array}{l}\text { (Z/E)-4-Ethyl-2,2-dimethyl-3- } \\
\text { octene }\end{array}$ & $\mathrm{C}_{12} \mathrm{H}_{24}$ & $1906.43,3.978$ & 168.1873 & 41350 & \\
\hline $\begin{array}{l}\text { 9-(E-2- } \\
\text { Propylcyclopropyl)nonan-1-ol }\end{array}$ & $\mathrm{C}_{15} \mathrm{H}_{30} \mathrm{O} ?$ & $1906.43,4.159$ & 226.2291 & 11201 & $1.36 \mathrm{E}-4$ \\
\hline $\begin{array}{l}\text { Ethyl 2,2,4-Trimethyl-5- } \\
\text { phenyl-4-pentenoate }\end{array}$ & $\mathrm{C}_{16} \mathrm{H}_{22} \mathrm{O}_{2} ?$ & $1918.44,2.741$ & 246.1614 & 20510 & 2.49E-4 \\
\hline Hentriacontane & $\mathrm{C}_{31} \mathrm{H}_{64}$ & $1924.44,4.664$ & 436.5003 & 102151 & \\
\hline $\begin{array}{l}\text { Decahydro-1,6- } \\
\text { dimethylnaphthalene }\end{array}$ & $\mathrm{C}_{12} \mathrm{H}_{22}$ & $1936.44,3.682$ & 166.1716 & 310887 & \\
\hline (2-Methylpropyl)cyclohexane & $\mathrm{C}_{10} \mathrm{H}_{20}$ & $1942.44,3.990$ & 140.156 & 78749 & \\
\hline $\begin{array}{l}\text { 3,7,7-Trimethyl-[1S- } \\
\text { (1à,2à,6à)]-bicyclo[4.1.0]hept- } \\
\text { 3-ene-2-thiol }\end{array}$ & $\mathrm{C}_{10} \mathrm{H}_{16} \mathrm{~S}$ & $1948.44,2.675$ & 168.0967 & 18248 & \\
\hline $\begin{array}{l}\text { 2,2-Dimethylanhydrosulfide } \\
\text { propanethioic acid }\end{array}$ & $\mathrm{C}_{10} \mathrm{H}_{18} \mathrm{O}_{2} \mathrm{~S}$ ? & $1948.44,4.690$ & 202.1022 & 18339 & $2.22 \mathrm{E}-4$ \\
\hline $\begin{array}{l}2-[1-(E)- \\
\text { Hexenyl]tetrahydrofuran }\end{array}$ & $\mathrm{C}_{10} \mathrm{H}_{18} \mathrm{O}$ ? & $1954.45,4.322$ & 154.1352 & 23786 & $2.88 \mathrm{E}-4$ \\
\hline n-Cetyl thiocyanate & $\mathrm{C}_{17} \mathrm{H}_{33} \mathrm{NS}$ & $1954.45,4.649$ & 283.2328 & 60263 & \\
\hline 1,2,3,4-Tetramethylbenzene & $\mathrm{C}_{10} \mathrm{H}_{14}$ & $1960.45,2.751$ & 134.109 & 202047 & \\
\hline $\begin{array}{l}\text { 5-Hydroxy-1,2,3,4- } \\
\text { tetrahydronaphthalen-1-one }\end{array}$ & $\mathrm{C}_{10} \mathrm{H}_{12} \mathrm{O}_{2} ?$ & $1960.45,3.675$ & 164.0832 & 29364 & $3.56 \mathrm{E}-4$ \\
\hline $\begin{array}{l}\text { Ethyl N-cyano-N- } \\
\text { pentylcarbamate }\end{array}$ & $\mathrm{C}_{9} \mathrm{H}_{16} \mathrm{~N}_{2} \mathrm{O}_{2}$ ? & $1960.45,4.315$ & 184.1206 & 42765 & 5.19E-4 \\
\hline 1-Hexadecene & $\mathrm{C}_{16} \mathrm{H}_{32}$ & $1972.45,4.264$ & 224.2499 & 8418 & \\
\hline 6-Methyldodecane & $\mathrm{C}_{13} \mathrm{H}_{28}$ & $1972.45,4.654$ & 184.2186 & 591451 & \\
\hline $\begin{array}{l}\text { 3-Ethyl-6-ethylidene-3-methyl- } \\
\text { 1,4-cyclohexadiene }\end{array}$ & $\mathrm{C}_{11} \mathrm{H}_{16}$ & $1984.45,2.873$ & 148.1247 & 17584 & \\
\hline $\begin{array}{l}1,2,3,5- \\
\text { Tetramethylcyclohexane }\end{array}$ & $\mathrm{C}_{10} \mathrm{H}_{20}$ & $1984.45,4.181$ & 140.156 & 227806 & \\
\hline $\begin{array}{l}\text { N-Methyl-2-(pyrrolidin-2'- } \\
\text { ylideneamino)propionamide }\end{array}$ & $\mathrm{C}_{8} \mathrm{H}_{15} \mathrm{~N}_{3} \mathrm{O}$ ? & $1984.45,4.283$ & 169.121 & 33211 & 4.03E-4 \\
\hline 2,4,6-Trimethylnonan-1-ol & $\mathrm{C}_{12} \mathrm{H}_{26} \mathrm{O} ?$ & $1990.46,4.197$ & 186.1978 & 37458 & 4.55E-4 \\
\hline 1,2,3,5-Tetramethyl benzene & $\mathrm{C}_{10} \mathrm{H}_{14}$ & $1996.46,2.715$ & 134.109 & 367655 & \\
\hline $\begin{array}{l}\text { 2,2,4-Trimethyl-4-pentenoic } \\
\text { acid }\end{array}$ & $\mathrm{C}_{8} \mathrm{H}_{14} \mathrm{O}_{2}$ ? & $1996.46,4.348$ & 142.0988 & 53960 & $6.55 \mathrm{E}-4$ \\
\hline 4-Methyldodecane & $\mathrm{C}_{13} \mathrm{H}_{28}$ & $2002.46,4.639$ & 184.2186 & 182346 & \\
\hline 2,3,7-Trimethyloctane & $\mathrm{C}_{11} \mathrm{H}_{24}$ & $2002.46,4.968$ & 156.1873 & 1910277 & \\
\hline 1-sec-Butyl-4-methylbenzene & $\mathrm{C}_{11} \mathrm{H}_{16}$ & $2020.47,2.859$ & 148.1247 & 105595 & \\
\hline $\begin{array}{l}\text { Decahydro-1,5- } \\
\text { dimethylnaphthalene }\end{array}$ & $\mathrm{C}_{12} \mathrm{H}_{22}$ & $2044.47,3.595$ & 166.1716 & 51022 & \\
\hline Hexacosane & $\mathrm{C}_{26} \mathrm{H}_{54}$ & $2044.47,4.870$ & 366.422 & 101393 & \\
\hline
\end{tabular}




\begin{tabular}{|c|c|c|c|c|c|}
\hline 3-Methyl-1-undecen-4-ol & $\mathrm{C}_{12} \mathrm{H}_{24} \mathrm{O} ?$ & $2050.47,4.061$ & 184.1822 & 12315 & $1.49 \mathrm{E}-4$ \\
\hline Cyclohexyl 4-cyanobutanoate & $\mathrm{C}_{11} \mathrm{H}_{17} \mathrm{NO}_{2} ?$ & $2056.48,3.407$ & 195.1254 & 10149 & $1.23 \mathrm{E}-4$ \\
\hline Hexylcyclohexane & $\mathrm{C}_{12} \mathrm{H}_{24}$ & $2086.48,3.929$ & 168.1873 & 366193 & \\
\hline $\begin{array}{l}\text { 3-(2'-Methyl-2'- } \\
\text { propenyl)cyclopentan-1-one }\end{array}$ & $\mathrm{C}_{9} \mathrm{H}_{14} \mathrm{O}$ & $2092.49,3.899$ & 138.1039 & 23002 & \\
\hline $\begin{array}{l}\text { 1-Butyl-2,4,6- } \\
\text { trimethylcyclohexane }\end{array}$ & $\mathrm{C}_{13} \mathrm{H}_{26}$ & $2104.49,4.078$ & 182.2029 & 126291 & \\
\hline $\begin{array}{l}\text { 1-(1,2-Dimethylcyclopentyl)- } \\
\text { trans-2-propyn-1-one }\end{array}$ & $\mathrm{C}_{10} \mathrm{H}_{14} \mathrm{O}$ ? & $2104.49,4.161$ & 150.1039 & 38360 & 4.65E-4 \\
\hline $\begin{array}{l}\text { (1S,8R)-1-Methylhexahydro- } \\
\text { 1H-pyrrolizine } \\
\text { [pseudoheliotridane] }\end{array}$ & $\mathrm{C}_{8} \mathrm{H}_{15} \mathrm{~N}$ & $2110.49,4.035$ & 125.1199 & 11858 & \\
\hline Butyl cyclobutyl oxaloate & $\mathrm{C}_{10} \mathrm{H}_{16} \mathrm{O}_{4}$ ? & $2116.49,4.105$ & 200.1043 & 24111 & 2.92E-4 \\
\hline 1,4-Dimethyl-cis-cyclooctane & $\mathrm{C}_{10} \mathrm{H}_{20}$ & $2122.49,4.058$ & 140.156 & 211355 & \\
\hline $\begin{array}{l}\text { [[2-(2-Propenyloxy)-1- } \\
\text { propenyl]sulfonyl]-(E)- } \\
\text { benzene }\end{array}$ & $\mathrm{C}_{12} \mathrm{H}_{14} \mathrm{O}_{3} \mathrm{~S} ?$ & $2134.5,4.113$ & 238.0658 & 27814 & 3.37E-4 \\
\hline $\begin{array}{l}\text { 2,2-Dimethyl-1-cycloheptane- } \\
\text { 3-buten-1-ol }\end{array}$ & $\mathrm{C}_{12} \mathrm{H}_{22} \mathrm{O}$ ? & $2140.5,3.871$ & 182.1665 & 27801 & 3.37E-4 \\
\hline $\begin{array}{l}\text { N-Allyl-2- } \\
\text { (methylthio)allylamine }\end{array}$ & $\mathrm{C}_{7} \mathrm{H}_{13} \mathrm{NS}$ & $2146.5,4.180$ & 143.0763 & 11940 & \\
\hline $\begin{array}{l}\text { cis,trans-2- } \\
\text { Ethylbicyclo[4.4.0]decane }\end{array}$ & $\mathrm{C}_{12} \mathrm{H}_{22}$ & $2158.5,3.564$ & 166.1716 & 158582 & \\
\hline $\begin{array}{l}\text { Bicyclo[3.2.2]nona-6,8-dien-3- } \\
\text { one, 6,8-dimethyl- }\end{array}$ & $\mathrm{C}_{11} \mathrm{H}_{14} \mathrm{O}$ & $2170.51,2.988$ & 162.1039 & 42486 & \\
\hline 1-(2-Ethylphenyl)ethan-1-one & $\mathrm{C}_{10} \mathrm{H}_{12} \mathrm{O}$ & $2176.51,2.762$ & 148.0883 & 7291 & \\
\hline 1,4-Dimethylcyclooctane & $\mathrm{C}_{10} \mathrm{H}_{20}$ & $2176.51,4.317$ & 140.156 & 77392 & \\
\hline $\begin{array}{l}\text { 3,3,4-Trimethyl- } \\
\text { benzeneacetaldehyde }\end{array}$ & $\mathrm{C}_{11} \mathrm{H}_{14} \mathrm{O}$ & $2200.52,2.937$ & 162.1039 & 27283 & \\
\hline $\begin{array}{l}\text { trans-2- } \\
\text { Ethylbicyclo[4.4.0]decane }\end{array}$ & $\mathrm{C}_{12} \mathrm{H}_{22}$ & $2224.52,3.511$ & 166.1716 & 83470 & \\
\hline Cyclotetradecane & $\mathrm{C}_{14} \mathrm{H}_{28}$ & $2224.52,4.181$ & 196.2186 & 197594 & \\
\hline 1-Methyl-2,3-dihydroindene & $\mathrm{C}_{10} \mathrm{H}_{12}$ & $2230.53,2.549$ & 132.0934 & 34520 & \\
\hline $\begin{array}{l}\text { Neopentyl 2,2- } \\
\text { dimethylpropanoate }\end{array}$ & $\mathrm{C}_{10} \mathrm{H}_{20} \mathrm{O}_{2} ?$ & $2260.53,4.697$ & 172.1458 & 74573 & $9.05 \mathrm{E}-4$ \\
\hline $\begin{array}{l}\text { 5-Hexyl-3,3- } \\
\text { dimethylcyclopentene }\end{array}$ & $\mathrm{C}_{13} \mathrm{H}_{24}$ & $2266.54,3.708$ & 180.1873 & 117100 & \\
\hline $\begin{array}{l}\text { trans-1-Methyl-2-nonyl- } \\
\text { cyclohexane }\end{array}$ & $\mathrm{C}_{16} \mathrm{H}_{32}$ & $2266.54,4.123$ & 224.2499 & 121820 & \\
\hline $\begin{array}{l}\text { Isopropyl O- } \\
\text { (isobutoxycarbonyl)-2,3,4- } \\
\text { trideoxy-4-methyl-D-erythro- } \\
\text { hex-2-enopyranoside }\end{array}$ & $\mathrm{C}_{15} \mathrm{H}_{26} \mathrm{O}_{5}$ ? & $2272.54,3.737$ & 286.1775 & 14008 & $1.70 \mathrm{E}-4$ \\
\hline $\begin{array}{l}\text { 1-Ethyl-4-(1-methylethyl)- } \\
\text { benzene }\end{array}$ & $\mathrm{C}_{11} \mathrm{H}_{16}$ & $2308.55,2.771$ & 148.1247 & 41320 & \\
\hline 2,2-Dimethylindan & $\mathrm{C}_{11} \mathrm{H}_{14}$ & $2320.55,2.684$ & 146.109 & 28063 & \\
\hline $\begin{array}{l}\text { 2,3-Dihydro-1,5,7-trimethyl- } \\
\text { 1H-indene }\end{array}$ & $\mathrm{C}_{12} \mathrm{H}_{16}$ & $2320.55,2.800$ & 160.1247 & 44858 & \\
\hline $1,2,3,4$-Tetrahydronaphthalene & $\mathrm{C}_{10} \mathrm{H}_{12}$ & $2332.55,2.479$ & 132.0934 & 31092 & \\
\hline
\end{tabular}




\begin{tabular}{|c|c|c|c|c|c|}
\hline $\begin{array}{l}\text { 2,3-Dihydro-1,6-dimethyl-1H- } \\
\text { indene }\end{array}$ & $\mathrm{C}_{11} \mathrm{H}_{14}$ & $2332.55,2.664$ & 146.109 & 63294 & \\
\hline Tetraethylbutanedinitrile & $\mathrm{C}_{12} \mathrm{H}_{20} \mathrm{~N}_{2}$ & $2332.55,3.977$ & 192.1621 & 32388 & \\
\hline Cyclobutyl m-toluate & $\mathrm{C}_{12} \mathrm{H}_{14} \mathrm{O}_{2}$ ? & $2344.56,2.923$ & 190.0988 & 26335 & $3.19 \mathrm{E}-4$ \\
\hline 3-Methyl-(E)- 4-nonen-1-ol & $\mathrm{C}_{10} \mathrm{H}_{20} \mathrm{O}$ ? & $2350.56,3.632$ & 156.1509 & 16079 & $1.18 \mathrm{E}-4$ \\
\hline (E)-7-Methyl-5-octenoic acid & $\mathrm{C}_{9} \mathrm{H}_{16} \mathrm{O}_{2}$ ? & $2350.56,4.353$ & 156.1145 & 32223 & 3.91E-4 \\
\hline 1-Octadecanol & $\mathrm{C}_{18} \mathrm{H}_{38} \mathrm{O}$ & $2362.56,3.998$ & 270.2917 & 49192 & \\
\hline 6-Methyl tridecane & $\mathrm{C}_{14} \mathrm{H}_{30}$ & $2368.56,4.603$ & 198.2342 & 381879 & \\
\hline (E)-2-Phenyl-2-pentene & $\mathrm{C}_{11} \mathrm{H}_{14}$ & $2374.57,2.640$ & 146.109 & 16766 & \\
\hline $\begin{array}{l}\text { 2,4-Diethyl-1- } \\
\text { methylcyclohexane }\end{array}$ & $\mathrm{C}_{11} \mathrm{H}_{22}$ & $2374.57,4.119$ & 154.1716 & 374027 & \\
\hline $\begin{array}{l}\text { 1,3-Dimethyl-5-(1- } \\
\text { methylethyl)- benzene }\end{array}$ & $\mathrm{C}_{11} \mathrm{H}_{16}$ & $2380.57,2.726$ & 148.1247 & 70146 & \\
\hline $\begin{array}{l}\text { 2,3-Dihydro-1,1,4-trimethyl- } \\
\text { 1H-indene }\end{array}$ & $\mathrm{C}_{12} \mathrm{H}_{16}$ & $2380.57,2.775$ & 160.1247 & 55692 & \\
\hline $\begin{array}{l}\text { (R,Z)-12-Hydroxy-9- } \\
\text { octadecenoic acid }\end{array}$ & $\mathrm{C}_{18} \mathrm{H}_{34} \mathrm{O}_{3} ?$ & $2380.57,3.750$ & 298.2502 & 24648 & 2.99E-4 \\
\hline $\begin{array}{l}\text { Decyl hexyl ester sulfurous } \\
\text { acid }\end{array}$ & $\mathrm{C}_{16} \mathrm{H}_{34} \mathrm{O}_{3} \mathrm{~S}$ & $2380.57,4.598$ & 306.2223 & 141494 & \\
\hline $\begin{array}{l}\text { 2,3-Dihydro-1,1-dimethyl-1H- } \\
\text { indene }\end{array}$ & $\mathrm{C}_{11} \mathrm{H}_{14}$ & $2386.57,2.642$ & 146.109 & 39127 & \\
\hline Pentyl undecyl sulfite & $\mathrm{C}_{16} \mathrm{H}_{34} \mathrm{O}_{3} \mathrm{~S}$ ? & $2398.57,4.593$ & 306.2223 & 203498 & $2.47 \mathrm{E}-3$ \\
\hline Vinyl neopentanoate & $\mathrm{C}_{8} \mathrm{H}_{14} \mathrm{O}_{2}$ ? & $2404.58,4.856$ & 142.0988 & 92620 & $1.12 \mathrm{E}-3$ \\
\hline $\begin{array}{l}\left(1 \mathrm{R}^{*}, 5 \mathrm{R}^{*}-5-\text {-Methyl-1- }\right. \\
\text { hydroxybicyclo[3.3.1] octane-3- } \\
\text { one }\end{array}$ & $\mathrm{C}_{10} \mathrm{H}_{16} \mathrm{O}_{2} ?$ & $2416.58,4.188$ & 168.1145 & 10467 & $1.27 \mathrm{E}-4$ \\
\hline 2-Methyltridecane & $\mathrm{C}_{14} \mathrm{H}_{30}$ & $2416.58,4.608$ & 198.2342 & 402203 & \\
\hline $\begin{array}{l}\text { 3-Acetyl-3- } \\
\text { methylcyclohexanone }\end{array}$ & $\mathrm{C}_{9} \mathrm{H}_{14} \mathrm{O}_{2}$ ? & $2422.58,4.204$ & 154.0988 & 17832 & $2.16 \mathrm{E}-4$ \\
\hline Cyclohexadecane & $\mathrm{C}_{16} \mathrm{H}_{32}$ & $2434.58,4.191$ & 224.2499 & 52464 & \\
\hline Heptadecane & $\mathrm{C}_{17} \mathrm{H}_{36}$ & $2434.58,4.783$ & 240.2812 & 1575180 & \\
\hline Nonadecane & $\mathrm{C}_{19} \mathrm{H}_{40}$ & $2440.59,4.509$ & 268.3125 & 38686 & \\
\hline 2,9-Dimethyldecane & $\mathrm{C}_{12} \mathrm{H}_{26}$ & $2458.59,4.531$ & 170.2029 & 359191 & \\
\hline $\begin{array}{l}\text { Methyl (1,4-dihydro-4-oxo-1- } \\
\text { hydroxynaphthalen-1-yl)- } \\
\text { acetate }\end{array}$ & $\mathrm{C}_{13} \mathrm{H}_{12} \mathrm{O}_{4}$ ? & $2464.59,2.911$ & 232.073 & 10382 & $1.26 \mathrm{E}-4$ \\
\hline 3-Acetoxyfuro[3,2-b]pyridine & $\mathrm{C}_{9} \mathrm{H}_{7} \mathrm{NO}_{3} ?$ & $2464.59,3.180$ & 177.042 & 10443 & $1.26 \mathrm{E}-4$ \\
\hline $\begin{array}{l}\text { S-Butyl } \\
\text { cyclohexanecarbothioate }\end{array}$ & $\mathrm{C}_{11} \mathrm{H}_{20} \mathrm{OS}$ ? & $2470.59,4.237$ & 200.1229 & 34524 & 4.19E-4 \\
\hline $\begin{array}{l}\text { 2-(2',2'-Dimethyl-1',3'-dioxan- } \\
\text { 4'-yl)-ethanol }\end{array}$ & $\mathrm{C}_{8} \mathrm{H}_{16} \mathrm{O}_{3}$ ? & $2476.6,2.783$ & 160.1094 & 13747 & $1.66 \mathrm{E}-4$ \\
\hline $\begin{array}{l}\text { Bicyclo[3.2.2]nona-6,8-dien-3- } \\
\text { one, 1,5-dimethyl- }\end{array}$ & $\mathrm{C}_{11} \mathrm{H}_{14} \mathrm{O}$ ? & $2500.6,2.903$ & 162.1039 & 21035 & $2.55 \mathrm{E}-4$ \\
\hline
\end{tabular}




\begin{tabular}{|c|c|c|c|c|c|}
\hline 3-Phenylcyclobutanol & $\mathrm{C}_{10} \mathrm{H}_{12} \mathrm{O}$ ? & $2506.6,2.581$ & 148.0883 & 8214 & $9.87 \mathrm{E}-5$ \\
\hline Heptylcyclohexane & $\mathrm{C}_{13} \mathrm{H}_{26}$ & $2506.6,3.909$ & 182.2029 & 355250 & \\
\hline Propynoic acid amide & $\mathrm{C}_{3} \mathrm{H}_{3} \mathrm{NO}$ & $2506.6,3.950$ & 69.0209 & 9344 & \\
\hline $\begin{array}{l}\text { 1,2,3,4-Tetrahydro-1,8- } \\
\text { dimethylnaphthalene }\end{array}$ & $\mathrm{C}_{12} \mathrm{H}_{16}$ & $2524.61,2.738$ & 160.1247 & 18546 & \\
\hline 5,5-Dimethylfuran-2(5H)-one & $\mathrm{C}_{6} \mathrm{H}_{8} \mathrm{O}_{2}$ & $2548.62,4.074$ & 112.0519 & 19640 & \\
\hline (E)-9-Tricosene & $\mathrm{C}_{23} \mathrm{H}_{46}$ & $2566.62,4.040$ & 322.3594 & 38886 & \\
\hline 5,5-Dimethyl-2(5H)-furanone & $\mathrm{C}_{6} \mathrm{H}_{8} \mathrm{O}_{2}$ & $2566.62,4.094$ & 112.0519 & 17754 & \\
\hline Azulene & $\mathrm{C}_{10} \mathrm{H}_{8}$ & $2584.63,2.269$ & 128.0621 & 26643 & \\
\hline $\begin{array}{l}\text { 1,4-Dimethyl-2-(2- } \\
\text { methylpropyl)benzene }\end{array}$ & $\mathrm{C}_{12} \mathrm{H}_{18}$ & $2596.63,2.841$ & 162.1403 & 37716 & \\
\hline $\begin{array}{l}\text { (R)-4-Ethynyl-3,5,5- } \\
\text { trimethylcyclohex-3-enyl } \\
\text { acetate }\end{array}$ & $\mathrm{C}_{13} \mathrm{H}_{18} \mathrm{O}_{2}$ ? & $2632.64,2.604$ & 206.1301 & 14212 & $1.72 \mathrm{E}-4$ \\
\hline 2,3,4,5-Tetrahydropyridine & $\mathrm{C}_{5} \mathrm{H}_{9} \mathrm{~N}$ & $2644.64,4.146$ & 83.073 & 62311 & \\
\hline $\begin{array}{l}\text { 1-[(2'-Methylamino-1'- } \\
\text { naphthyl)methyl]-2-naphthol }\end{array}$ & $\mathrm{C}_{22} \mathrm{H}_{17} \mathrm{NO} ?$ & $2674.65,2.874$ & 311.1305 & 12239 & $1.48 \mathrm{E}-4$ \\
\hline $\begin{array}{l}\text { (2S,6R)-2,6-Dimethyl-1- } \\
\text { octanol }\end{array}$ & $\mathrm{C}_{10} \mathrm{H}_{22} \mathrm{O}$ ? & $2674.65,4.034$ & 158.1665 & 64799 & 7.87E-4 \\
\hline Tricosane & $\mathrm{C}_{23} \mathrm{H}_{48}$ & $2680.65,4.576$ & 324.3751 & 87128 & \\
\hline $\begin{array}{l}\text { 2,3-Dihydro-1,3-dimethyl-1H- } \\
\text { indene }\end{array}$ & $\mathrm{C}_{11} \mathrm{H}_{14}$ & $2698.66,2.592$ & 146.109 & 50864 & \\
\hline $\begin{array}{l}\text { 3-Oxo-1,3-dihydro-1- } \\
\text { isobenzofuranyl phenylacetate }\end{array}$ & $\mathrm{C}_{16} \mathrm{H}_{12} \mathrm{O}_{4}$ ? & $2716.66,2.767$ & 268.073 & 22995 & 2.79E-4 \\
\hline $\begin{array}{l}9,10,11- \\
\text { Trioxabicyclo[6.2.1]undecane }\end{array}$ & $\mathrm{C}_{8} \mathrm{H}_{14} \mathrm{O}_{3}$ & $2722.67,4.290$ & 158.0937 & 18792 & \\
\hline $\begin{array}{l}\text { 2,2,5,5-tetramethyl-3,4- } \\
\text { bis(methylene)cyclopent-1-yl } \\
\text { p-toluenesulfonate }\end{array}$ & $\mathrm{C}_{18} \mathrm{H}_{24} \mathrm{O}_{3} \mathrm{~S}$ ? & $2728.67,2.661$ & 320.1441 & 12921 & $1.56 \mathrm{E}-4$ \\
\hline 2-Methyl-1-pyrroline & $\mathrm{C}_{5} \mathrm{H}_{9} \mathrm{~N}$ & $2734.67,3.999$ & 83.073 & 38615 & \\
\hline $\begin{array}{l}\text { 1,2,3,4-Tetrahydro-5- } \\
\text { methylnaphthalene }\end{array}$ & $\mathrm{C}_{11} \mathrm{H}_{14}$ & $2746.67,2.530$ & 146.109 & 72507 & \\
\hline 4,4-Diethyl-2,5-octadiyne & $\mathrm{C}_{12} \mathrm{H}_{18}$ & $2752.68,2.932$ & 162.1403 & 14205 & \\
\hline Cyclopentanodec-5-ene & $\mathrm{C}_{15} \mathrm{H}_{28}$ & $2752.68,4.209$ & 208.2186 & 78730 & \\
\hline $\begin{array}{l}\text { 2,6,10,14- } \\
\text { Tetramethylheptadecane }\end{array}$ & $\mathrm{C}_{21} \mathrm{H}_{44}$ & $2752.68,4.773$ & 296.3438 & 1683818 & \\
\hline Cyclooctylhydroperoxide & $\mathrm{C}_{12} \mathrm{H}_{24} \mathrm{O}_{2}$ ? & $2758.68,4.144$ & 200.1771 & 20846 & $2.53 \mathrm{E}-4$ \\
\hline $\begin{array}{l}\text { spiro[4,5-Dihydro-3- } \\
\text { methylisoxazole-5,1'- } \\
\text { cyclopropane] }\end{array}$ & $\mathrm{C}_{6} \mathrm{H}_{9} \mathrm{NO} ?$ & $2764.68,4.241$ & 111.0679 & 18899 & $2.29 \mathrm{E}-4$ \\
\hline 5-Methyltetradecane & $\mathrm{C}_{15} \mathrm{H}_{32}$ & $2764.68,4.515$ & 212.2499 & 164941 & \\
\hline 4-Methyl- Tridecane & $\mathrm{C}_{14} \mathrm{H}_{30}$ & $2782.68,4.521$ & 198.2342 & 166757 & \\
\hline 1-Phenyl-1-undecyn-5-ol & $\mathrm{C}_{17} \mathrm{H}_{24} \mathrm{O}$ ? & $2788.69,2.817$ & 244.1822 & 8786 & $1.06 \mathrm{E}-4$ \\
\hline
\end{tabular}




\begin{tabular}{|c|c|c|c|c|c|}
\hline (E)-6-Tosyl-4-nonene & $\mathrm{C}_{16} \mathrm{H}_{24} \mathrm{O}_{2} \mathrm{~S}$ ? & $2794.69,4.056$ & 280.1492 & 104284 & $1.26 \mathrm{E}-3$ \\
\hline 3-Methyltridecane & $\mathrm{C}_{14} \mathrm{H}_{30}$ & $2800.69,4.526$ & 198.2342 & 437619 & \\
\hline 1,2,2-Trimethylindan & $\mathrm{C}_{12} \mathrm{H}_{16}$ & $2818.69,2.674$ & 160.1247 & 44767 & \\
\hline $\begin{array}{l}\text { endo-2,3- } \\
\text { Di(methoxycarbonyl)- } \\
\text { benzo[e]bicyclo[2.2.2]octane }\end{array}$ & $\mathrm{C}_{16} \mathrm{H}_{18} \mathrm{O}_{4}$ & $2824.7,2.793$ & 274.12 & 9665 & \\
\hline $\begin{array}{l}\text { 2-Methyl-, (E)-3-hexen-5-yn-2- } \\
\text { ol }\end{array}$ & $\mathrm{C}_{7} \mathrm{H}_{10} \mathrm{O}$ ? & $2836.7,3.606$ & 110.0726 & 19126 & 2.32E-4 \\
\hline $\begin{array}{l}2,6,10,15- \\
\text { Tetramethylheptadecane }\end{array}$ & $\mathrm{C}_{21} \mathrm{H}_{44}$ & $2836.7,4.474$ & 296.3438 & 308354 & \\
\hline $\begin{array}{l}\text { Methyl 3-methyl-2,4- } \\
\text { hexadienoate }\end{array}$ & $\mathrm{C}_{8} \mathrm{H}_{10} \mathrm{O}_{4}$ ? & $2842.7,4.153$ & 170.0574 & 7877 & $9.56 \mathrm{E}-5$ \\
\hline $\begin{array}{l}\text { 4-(Phenylthio)-2,4-dimethyl-2- } \\
\text { pentene }\end{array}$ & $\mathrm{C}_{13} \mathrm{H}_{18} \mathrm{~S}$ & $2848.7,3.838$ & 206.1124 & 27792 & \\
\hline $\begin{array}{l}\text { O-(tert-Butoxycarbonyl)-N- } \\
\text { isopropylidene oxime }\end{array}$ & $\mathrm{C}_{8} \mathrm{H}_{15} \mathrm{NO}_{3}$ & $2884.71,4.181$ & 173.1046 & 7946 & \\
\hline 1-Heptadecanol & $\mathrm{C}_{17} \mathrm{H}_{36} \mathrm{O}$ ? & $2896.72,3.911$ & 256.2761 & 94026 & $1.14 \mathrm{E}-3$ \\
\hline 1-Methoxybicyclo(3.2.1)octane & $\mathrm{C}_{9} \mathrm{H}_{16} \mathrm{O}$ & $2896.72,4.142$ & 140.1196 & 18753 & \\
\hline Octylcyclohexane & $\mathrm{C}_{14} \mathrm{H}_{28}$ & $2908.72,3.873$ & 196.2186 & 299890 & \\
\hline $\begin{array}{l}\text { 2,3-Dihydro-1,1,4,6- } \\
\text { tetramethyl-1H-indene }\end{array}$ & $\mathrm{C}_{13} \mathrm{H}_{18}$ & $2926.73,2.730$ & 174.1403 & 33243 & \\
\hline $\begin{array}{l}\text { 2,2-dimethyl-5-hexen-1-ol } \\
\text { tosylate }\end{array}$ & $\mathrm{C}_{15} \mathrm{H}_{23} \mathrm{O}_{3} \mathrm{~S} ?$ & $2926.73,4.132$ & 283.1362 & 24887 & $3.02 \mathrm{E}-4$ \\
\hline 1-Eicosanol & $\mathrm{C}_{20} \mathrm{H}_{42} \mathrm{O}$ ? & $2944.73,4.213$ & 298.323 & 90814 & $1.10 \mathrm{E}-4$ \\
\hline $\begin{array}{l}\text { 2,3-Dihydro-1,4,7-trimethyl- } \\
\text { 1H-indene }\end{array}$ & $\mathrm{C}_{12} \mathrm{H}_{16}$ & $2962.74,2.584$ & 160.1247 & 11279 & \\
\hline $\begin{array}{l}\text { 1,2-Dibutyl-trans- } \\
\text { cyclopropane }\end{array}$ & $\mathrm{C}_{11} \mathrm{H}_{22}$ & $2974.74,4.298$ & 154.1716 & 27741 & \\
\hline 1-Tetradecanol & $\mathrm{C}_{14} \mathrm{H}_{30} \mathrm{O}$ ? & $3016.75,4.102$ & 214.2291 & 135772 & 1.64E-4 \\
\hline 1-Ethylidene-1H-indene & $\mathrm{C}_{11} \mathrm{H}_{10}$ & $3022.75,2.319$ & 142.0777 & 165945 & \\
\hline 3-Methoxy-1-propyne & $\mathrm{C}_{4} \mathrm{H}_{6} \mathrm{O}$ & $3022.75,4.149$ & 70.0413 & 61177 & \\
\hline $\begin{array}{l}\text { 1-Methyl-3-(2- } \\
\text { methylpropyl)cyclopentane }\end{array}$ & $\mathrm{C}_{10} \mathrm{H}_{20}$ & $3040.76,4.047$ & 140.156 & 177458 & \\
\hline $\begin{array}{l}\text { 1-Octyl-1,4- } \\
\text { dihydronaphthalene }\end{array}$ & $\mathrm{C}_{18} \mathrm{H}_{26}$ & $3052.76,2.514$ & 242.2029 & 12070 & \\
\hline Hexadecane & $\mathrm{C}_{16} \mathrm{H}_{34}$ & $3070.77,4.461$ & 226.2655 & 85225 & \\
\hline 7-Methylpentadecane & $\mathrm{C}_{16} \mathrm{H}_{34}$ & $3106.78,4.469$ & 226.2655 & 505545 & \\
\hline $\begin{array}{l}\text { 3-Benzyl-4-ethyl-4-methyl- } \\
\text { oxetan-2-one }\end{array}$ & $\mathrm{C}_{13} \mathrm{H}_{16} \mathrm{O}_{2}$ ? & $3118.78,2.534$ & 204.1145 & 13478 & $1.63 \mathrm{E}-4$ \\
\hline $\begin{array}{l}\text { (E)-1-[2'-(3",4",4"- } \\
\text { Trimethylpent-1"- } \\
\text { yl)phenyl]ethanone }\end{array}$ & $\mathrm{C}_{16} \mathrm{H}_{22} \mathrm{O}$ ? & $3154.79,2.713$ & 230.1665 & 17376 & $2.11 \mathrm{E}-4$ \\
\hline $\begin{array}{l}\text { (1S,2R)-6,6-Dimethyl-2- } \\
\text { ethylbicyclo[3.1.1]heptan-3- } \\
\text { one }\end{array}$ & $\mathrm{C}_{11} \mathrm{H}_{18} \mathrm{O}$ ? & $3202.8,4.068$ & 166.1352 & 11060 & 1.34E-4 \\
\hline 2,6,8-Trimethyldecane & $\mathrm{C}_{13} \mathrm{H}_{28}$ & $3244.82,4.567$ & 184.2186 & 325128 & \\
\hline
\end{tabular}




\begin{tabular}{|c|c|c|c|c|c|}
\hline $\begin{array}{l}\text { 2-(1,1-Dimethylethyl)-3(2H)- } \\
\text { pyridazinone }\end{array}$ & $\mathrm{C}_{8} \mathrm{H}_{12} \mathrm{~N}_{2} \mathrm{O}$ & $3256.82,4.014$ & 152.0944 & 48529 & \\
\hline 5-Acetyllongipinandiolone & $\mathrm{C}_{17} \mathrm{H}_{26} \mathrm{O}_{4} ?$ & $3262.82,4.068$ & 294.1826 & 28325 & $3.44 \mathrm{E}-4$ \\
\hline $\begin{array}{l}\text { 1,2,3,4-Tetrahydro-5,7- } \\
\text { dimethylnaphthalene }\end{array}$ & $\mathrm{C}_{12} \mathrm{H}_{16}$ & $3274.82,2.506$ & 160.1247 & 73070 & \\
\hline $\begin{array}{l}\text { 1,2,3,4-Tetrahydro-1,1,6- } \\
\text { trimethylnaphthalene }\end{array}$ & $\mathrm{C}_{13} \mathrm{H}_{18}$ & $3274.82,2.693$ & 174.1403 & 21535 & \\
\hline $\begin{array}{l}\text { 1,3-Dimethyl-2-pentylidene } \\
\text { cyclohexanecarboxaldehyde }\end{array}$ & $\mathrm{C}_{14} \mathrm{H}_{24} \mathrm{O}$ ? & $3286.83,3.754$ & 208.1822 & 12501 & $1.51 \mathrm{E}-4$ \\
\hline $\begin{array}{l}\text { (5S,7S,9S,E)-S-Ethyl 5,7,9- } \\
\text { trimethyltridec-3-enethioate }\end{array}$ & $\mathrm{C}_{18} \mathrm{H}_{34} \mathrm{OS}$ ? & $3298.83,4.063$ & 298.2325 & 50733 & $6.16 \mathrm{E}-4$ \\
\hline $\begin{array}{l}\text { 1-Cyano-2,6-dimethyl-1,5- } \\
\text { heptadien-3-ol }\end{array}$ & $\mathrm{C}_{10} \mathrm{H}_{15} \mathrm{NO} ?$ & $3304.83,4.257$ & 165.1148 & 11847 & $1.43 \mathrm{E}-4$ \\
\hline 1-Ethylnaphthalene & $\mathrm{C}_{12} \mathrm{H}_{12}$ & $3382.86,2.367$ & 156.0934 & 30497 & \\
\hline 1,7-Dimethylnaphthalene & $\mathrm{C}_{12} \mathrm{H}_{12}$ & $3418.87,2.386$ & 156.0934 & 272739 & \\
\hline $\begin{array}{l}\text { Methyl 3-(p- } \\
\text { nitrophenyl)bicyclo[1.1.1]penta } \\
\text { ne-1-carboxylate }\end{array}$ & $\mathrm{C}_{13} \mathrm{H}_{13} \mathrm{NO}_{4}$ & $3430.87,2.536$ & 247.0839 & 7990 & \\
\hline Tritriacontane & $\mathrm{C}_{33} \mathrm{H}_{68}$ & $3454.88,4.397$ & 464.5316 & 429391 & \\
\hline $\begin{array}{l}\text { 3,6-Dihydro-4-phenyl-1-oxide- } \\
\text { 2H-thiopyran }\end{array}$ & $\mathrm{C}_{11} \mathrm{H}_{12} \mathrm{OS}$ & $3466.88,2.524$ & 192.0603 & 9899 & \\
\hline (E)-3-Octen-2,5-dione & $\mathrm{C}_{8} \mathrm{H}_{12} \mathrm{O}_{2}$ & $3478.88,3.918$ & 140.0832 & 57040 & \\
\hline 3,8-Dimethylundecane & $\mathrm{C}_{13} \mathrm{H}_{28}$ & $3496.89,4.377$ & 184.2186 & 142538 & \\
\hline 1,6-Dimethylnaphthalene & $\mathrm{C}_{12} \mathrm{H}_{12}$ & $3502.89,2.345$ & 156.0934 & 122237 & \\
\hline $\begin{array}{l}\text { 1-Phenyl-2,3- } \\
\text { diazabicyclo[2.2.1]hept-2-ene }\end{array}$ & $\mathrm{C}_{11} \mathrm{H}_{12} \mathrm{~N}_{2}$ & $3520.9,2.475$ & 172.0995 & 21334 & \\
\hline 2,6,11-Trimethyldodecane & $\mathrm{C}_{15} \mathrm{H}_{32}$ & $3586.91,4.742$ & 212.2499 & 1839748 & \\
\hline 1,2-Dimethylnaphthalene & $\mathrm{C}_{12} \mathrm{H}_{12}$ & $3622.92,2.305$ & 156.0934 & 21643 & \\
\hline Undecylcyclohexane & $\mathrm{C}_{17} \mathrm{H}_{34}$ & $3652.93,3.811$ & 238.2655 & 201593 & \\
\hline 1,2-Epoxy-3-tetradecanol & $\mathrm{C}_{14} \mathrm{H}_{28} \mathrm{O}_{2}$ ? & $3718.95,3.971$ & 228.2084 & 33183 & 4.03E-4 \\
\hline $\begin{array}{l}\text { 2-(1,3-Dioxolan-2-yl)-1-(2- } \\
\text { furyl)ethane }\end{array}$ & $\mathrm{C}_{9} \mathrm{H}_{10} \mathrm{O}_{3}$ ? & $3724.95,4.387$ & 166.0624 & 46807 & $5.68 \mathrm{E}-4$ \\
\hline $\begin{array}{l}\text { 3,5-trans-3-Hydroxy-5-[(E)-1- } \\
\text { pentenyl]-4,5-dihydro-2(3H)- } \\
\text { furanone }\end{array}$ & $\mathrm{C}_{9} \mathrm{H}_{14} \mathrm{O}_{3}$ ? & $3748.96,4.015$ & 170.0937 & 37678 & $4.57 \mathrm{E}-4$ \\
\hline 2-(1-Methylethyl)naphthalene & $\mathrm{C}_{13} \mathrm{H}_{14}$ & $3754.96,2.433$ & 170.109 & 32394 & \\
\hline 1,4,5-Trimethylnaphthalene & $\mathrm{C}_{13} \mathrm{H}_{14}$ & $3766.97,2.418$ & 170.109 & 46919 & \\
\hline 4-Methyl-1,1'-biphenyl & $\mathrm{C}_{13} \mathrm{H}_{12}$ & $3772.97,2.271$ & 168.0934 & 26430 & \\
\hline Heneicosane & $\mathrm{C}_{21} \mathrm{H}_{44}$ & $3826.98,4.339$ & 296.3438 & 136147 & \\
\hline $\begin{array}{l}\text { 5,7-Dimethyl-1,4-dihydro-1,4- } \\
\text { methanonaphthalene }\end{array}$ & $\mathrm{C}_{13} \mathrm{H}_{14}$ & $3844.99,2.358$ & 170.109 & 14779 & \\
\hline $\begin{array}{l}\text { endo-7-(3-Carbethoxy-3- } \\
\text { butenyl)-8-hexyl-2,9- } \\
\text { dioxabicyclo[4,3,0]nonane }\end{array}$ & $\mathrm{C}_{20} \mathrm{H}_{34} \mathrm{O}_{4}$ ? & $3850.99,4.254$ & 338.2452 & 21742 & $2.64 \mathrm{E}-4$ \\
\hline
\end{tabular}




\begin{tabular}{|c|c|c|c|c|c|}
\hline $\begin{array}{l}\text { 4-(2- } \\
\text { Tetrahydropyranyl)oxybutanal }\end{array}$ & $\mathrm{C}_{9} \mathrm{H}_{16} \mathrm{O}_{3} ?$ & $3947.02,4.410$ & 172.1094 & 28861 & $3.50 \mathrm{E}-4$ \\
\hline 1,4,6-Trimethylnaphthalene & $\mathrm{C}_{13} \mathrm{H}_{14}$ & $3965.02,2.381$ & 170.109 & 194557 & \\
\hline $\begin{array}{l}\text { (2E)-1-O-Acetyl-3,7-dimethyl- } \\
\text { 2-octen-1-ol 6,7-epoxide }\end{array}$ & $\mathrm{C}_{12} \mathrm{H}_{20} \mathrm{O}_{3}$ ? & $4103.06,4.299$ & 212.1407 & 36585 & 4.44E-4 \\
\hline n-Octyl ethynyl ether & $\mathrm{C}_{10} \mathrm{H}_{18} \mathrm{O}$ ? & $4109.06,4.292$ & 154.1352 & 74433 & $9.04 \mathrm{E}-4$ \\
\hline 4-Methylundecane & $\mathrm{C}_{12} \mathrm{H}_{26}$ & $4145.07,4.281$ & 170.2029 & 63718 & \\
\hline $\begin{array}{l}\text { 2-(Hydroxymethyl)-5- } \\
\text { hexyltetrahydrofuran }\end{array}$ & $\mathrm{C}_{11} \mathrm{H}_{22} \mathrm{O}_{2}$ ? & $4157.08,2.431$ & 186.1614 & 8459 & $1.02 \mathrm{E}-4$ \\
\hline Dehydrochamazulene & $\mathrm{C}_{14} \mathrm{H}_{14}$ & $4163.08,2.315$ & 182.109 & 16743 & \\
\hline $\begin{array}{l}\text { (2S)-2-(1-Ethyl-1- } \\
\text { methoxypropyl)-N-((E)-1-\{[(3- } \\
\text { methyl-2- } \\
\text { butenyl)oxy]methyl\}propyliden } \\
\text { e)-1-pyrrolidinamine }\end{array}$ & $\mathrm{C}_{19} \mathrm{H}_{36} \mathrm{~N}_{2} \mathrm{O}_{2} ?$ & $4175.08,4.106$ & 324.2771 & 24984 & 3.03E-4 \\
\hline Neopentyl 2-oxobutanoate & $\mathrm{C}_{9} \mathrm{H}_{16} \mathrm{O}_{3}$ ? & $4193.09,4.263$ & 172.1094 & 151023 & $1.83 \mathrm{E}-3$ \\
\hline (E)-Deca-1,6-dien-3-one & $\mathrm{C}_{10} \mathrm{H}_{16} \mathrm{O}$ ? & $4211.09,4.524$ & 152.1196 & 6853 & $8.32 \mathrm{E}-5$ \\
\hline $\begin{array}{l}2,3,3,6,6,7-\text { Hexamethyl-1,4,7- } \\
\text { octatriene }\end{array}$ & $\mathrm{C}_{14} \mathrm{H}_{24}$ & $4223.1,3.974$ & 192.1873 & 23453 & \\
\hline $\begin{array}{l}((\mathrm{Z}) \text {-Hept-5-ene-1,3-diynyl)- } \\
\text { benzene }\end{array}$ & $\mathrm{C}_{13} \mathrm{H}_{10}$ & $4253.11,2.202$ & 166.0777 & 11613 & \\
\hline 3-Ethylcosane & $\mathrm{C}_{22} \mathrm{H}_{46}$ & $4265.11,4.519$ & 310.3594 & 65264 & \\
\hline Heptacosane & $\mathrm{C}_{27} \mathrm{H}_{56}$ & $4289.12,4.202$ & 380.4377 & 2063047 & \\
\hline 2,2-Dimethyl-3-heptanone & $\mathrm{C}_{9} \mathrm{H}_{18} \mathrm{O}$ ? & $4307.12,4.458$ & 142.1352 & 19587 & $2.37 \mathrm{E}-4$ \\
\hline $\begin{array}{l}\text { 3-(1,1-Dimethylethoxy)-1- } \\
\text { propene }\end{array}$ & $\mathrm{C}_{7} \mathrm{H}_{14} \mathrm{O}$ & $4307.12,4.556$ & 114.1039 & 16529 & \\
\hline $1,4,5,8$-Tetramethylnaphthalene & $\mathrm{C}_{14} \mathrm{H}_{16}$ & $4367.14,2.417$ & 184.1247 & 53765 & \\
\hline (Dodecyloxy)methyl isonitrile & $\mathrm{C}_{14} \mathrm{H}_{27} \mathrm{NO} ?$ & $4385.14,3.937$ & 225.2087 & 107405 & $1.30 \mathrm{E}-3$ \\
\hline $\begin{array}{l}\text { Methyl (1RS,3RS,4SR)-3,4- } \\
\text { epoxycyclohexanecarboxylate }\end{array}$ & $\mathrm{C}_{8} \mathrm{H}_{12} \mathrm{O}_{3}$ ? & $4475.17,3.838$ & 156.0781 & 35088 & $4.26 \mathrm{E}-4$ \\
\hline Tetracosane & $\mathrm{C}_{24} \mathrm{H}_{50}$ & $4493.17,4.224$ & 338.3907 & 121644 & \\
\hline Heptane & $\mathrm{C}_{7} \mathrm{H}_{16}$ & $4505.18,4.491$ & 100.1247 & 43187 & \\
\hline $\begin{array}{l}\text { 2H-3,4-Dihydro-5- } \\
\text { methylpyrrole }\end{array}$ & $\mathrm{C}_{4} \mathrm{H}_{11} \mathrm{~N}$ & $4601.21,3.792$ & 73.0886 & 26459 & \\
\hline 5,5-Diphenylpentanoic acid & $\mathrm{C}_{17} \mathrm{H}_{18} \mathrm{O}_{2} ?$ & $4613.21,2.244$ & 254.1301 & 24524 & $2.97 \mathrm{E}-4$ \\
\hline 2-Methylene-3-butenylamine & $\mathrm{C}_{5} \mathrm{H}_{9} \mathrm{~N}$ & $4625.21,3.763$ & 83.073 & 69726 & \\
\hline 9-Methyl-9H-fluorene & $\mathrm{C}_{14} \mathrm{H}_{12}$ & $4649.22,2.235$ & 180.0934 & 34450 & \\
\hline cis-Bicyclo[3.3.0] octan-1-ol & $\mathrm{C}_{8} \mathrm{H}_{14} \mathrm{O}$ ? & $4679.23,3.952$ & 126.1039 & 34498 & 4.19E-4 \\
\hline 2,3-Dimethyl-9H-fluorene & $\mathrm{C}_{15} \mathrm{H}_{14}$ & $5087.35,2.253$ & 194.109 & 7495 & \\
\hline $\begin{array}{l}\text { 1,4-Bis(cyclohex-1-enyl)buta- } \\
\text { 1,3-diyne }\end{array}$ & $\mathrm{C}_{16} \mathrm{H}_{18}$ & $5207.38,4.235$ & 210.1403 & 40009 & \\
\hline
\end{tabular}




\begin{tabular}{|c|c|c|c|c|c|}
\hline $\begin{array}{l}\text { 3-tert-Butyl-1,2-dimethoxy-5- } \\
\text { methylbenzene }\end{array}$ & $\mathrm{C}_{13} \mathrm{H}_{20} \mathrm{O}_{2}$ ? & $5255.39,2.359$ & 208.1458 & 21832 & $2.65 \mathrm{E}-4$ \\
\hline $\begin{array}{l}\text { 1,4-Dihydro-4,4-dimethyl-1,3- } \\
\text { diphenylisoquinoline }\end{array}$ & $\mathrm{C}_{23} \mathrm{H}_{21} \mathrm{~N}$ & $5273.4,2.353$ & 311.1669 & 10750 & \\
\hline $\begin{array}{l}\text { 2-Ethyl-(1à,2à,5à)-6- } \\
\text { oxabicyclo[3.1.0]hexan-2-ol }\end{array}$ & $\mathrm{C}_{7} \mathrm{H}_{12} \mathrm{O}_{2}$ ? & $5279.4,4.162$ & 128.0832 & 42444 & $5.15 \mathrm{E}-4$ \\
\hline $\begin{array}{l}\text { 9,10-Dihydro-9,10-dimethyl- } \\
\text { anthracene }\end{array}$ & $\mathrm{C}_{16} \mathrm{H}_{16}$ & $5291.4,2.349$ & 208.1247 & 17524 & \\
\hline $\begin{array}{l}\text { 6a,10b-Dihydro-6a,10b- } \\
\text { dimethyl-trans- } \\
\text { cyclopentaheptalene }\end{array}$ & $\mathrm{C}_{16} \mathrm{H}_{16}$ & $5321.41,2.329$ & 208.1247 & 9000 & \\
\hline 7-Phenylindene & $\mathrm{C}_{15} \mathrm{H}_{12}$ & $5459.45,2.164$ & 192.0934 & 26744 & \\
\hline $\begin{array}{l}\text { 9,10-Dihydro-3,6- } \\
\text { dimethylphenanthrene }\end{array}$ & $\mathrm{C}_{16} \mathrm{H}_{16}$ & $5465.45,2.331$ & 208.1247 & 9621 & \\
\hline 2,7-Dimethylphenanthrene & $\mathrm{C}_{16} \mathrm{H}_{14}$ & $5669.51,2.233$ & 206.109 & 9378 & \\
\hline 3,6-Dimethylphenanthrene & $\mathrm{C}_{16} \mathrm{H}_{14}$ & $5717.53,2.235$ & 206.109 & 73731 & \\
\hline $\begin{array}{l}\text { 2-(2-Oxopropyl)- } \\
\text { cyclopentanone }\end{array}$ & $\mathrm{C}_{8} \mathrm{H}_{12} \mathrm{O}_{2}$ ? & $5729.53,3.751$ & 140.0832 & 29634 & $3.59 \mathrm{E}-4$ \\
\hline 2,2-Dimethylpentane & $\mathrm{C}_{7} \mathrm{H}_{16}$ & $5801.55,4.235$ & 100.1247 & 27923 & \\
\hline $\begin{array}{l}\text { (3R)-3-Phenyl-2,3-dihydro-1H- } \\
\text { isoindol-1-one }\end{array}$ & $\mathrm{C}_{14} \mathrm{H}_{11} \mathrm{NO} ?$ & $6563.77,1.096$ & 209.0835 & 743078 & $9.02 \mathrm{E}-3$ \\
\hline $\begin{array}{l}\text { cis- and trans-3,6-Dicetoxy-1- } \\
\text { cyclohexene }\end{array}$ & $\mathrm{C}_{10} \mathrm{H}_{14} \mathrm{O}_{4}$ ? & $6971.89,1.084$ & 198.0887 & 9769 & $1.18 \mathrm{E}-4$ \\
\hline 4-Methylhexanoic acid & $\mathrm{C}_{7} \mathrm{H}_{14} \mathrm{O}_{2}$ ? & $7325.99,4.679$ & 130.0988 & 51542 & $6.26 \mathrm{E}-4$ \\
\hline $\begin{array}{l}\text { (1S,2S)-N-Amino-2- } \\
\text { methylamino-1-phenyl-1- } \\
\text { propanol }\end{array}$ & $\mathrm{C}_{11} \mathrm{H}_{16} \mathrm{~N}_{2} \mathrm{O}$ ? & $7349.99,1.158$ & 192.1257 & 30680 & 3.72E-4 \\
\hline $\begin{array}{l}\text { 5,10-Dihydro-4H- } \\
\text { cyclopenta[b]thieno[6,5- } \\
\text { b]quinoline-4,5-dione }\end{array}$ & $\mathrm{C}_{14} \mathrm{H}_{7} \mathrm{NO}_{2} \mathrm{~S}$ ? & $7698.09,1.712$ & 253.0192 & 101633 & $1.23 \mathrm{E}-3$ \\
\hline $\begin{array}{l}\text { 1-(6,6-Dicyano-1,3,5- } \\
\text { hexatrienyl)-1,3,5- } \\
\text { cycloheptatriene }\end{array}$ & $\mathrm{C}_{15} \mathrm{H}_{12} \mathrm{~N}_{2}$ & $7884.15,0.648$ & 220.0995 & 21032 & \\
\hline $\begin{array}{l}\text { 5-(Pyridine-4-yl)-1,3,4- } \\
\text { oxadiazole-2-(3H)-thione }\end{array}$ & $\mathrm{C}_{7} \mathrm{H}_{5} \mathrm{~N}_{3} \mathrm{OS}$ & $7902.15,1.204$ & 179.0148 & 12351 & \\
\hline $\begin{array}{l}\text { Ethyl N-[(9- } \\
\text { fluorenyl)methoxycarbonyl]- } \\
\text { DL-dithioalanine }\end{array}$ & $\mathrm{C}_{20} \mathrm{H}_{21} \mathrm{NO}_{2} \mathrm{~S}_{2} ?$ & $8064.2,1.208$ & 371.1008 & 11800 & $1.43 \mathrm{E}-4$ \\
\hline $\begin{array}{l}\text { (S)-(E)-4-Acetoxy-1-phenyl-2- } \\
\text { dodecen-1-one }\end{array}$ & $\mathrm{C}_{20} \mathrm{H}_{28} \mathrm{O}_{3}$ ? & $8160.23,1.111$ & 316.2033 & 135274 & $1.64 \mathrm{E}-3$ \\
\hline $\begin{array}{l}\text { [(Methylbenzyl)oxy](methoxy) } \\
\text { methyl }\end{array}$ & $\mathrm{C}_{10} \mathrm{H}_{14} \mathrm{O}_{2}$ ? & $8196.24,1.080$ & 166.0988 & 10058 & $1.22 \mathrm{E}-4$ \\
\hline $\begin{array}{l}\text { 3,4-Dihydro-2,4-diphenyl-7,12- } \\
\text { methanocyclodeca[b]pyridine }\end{array}$ & $\mathrm{C}_{27} \mathrm{H}_{21} \mathrm{~N}$ & $8196.24,1.285$ & 359.1669 & 16551 & \\
\hline
\end{tabular}


Table 2S16. Data obtained for the distillate fraction of condensate-like oil 5 by using GCxGC/(+EI)-TOF MS. Data provided are based on ChromaTOF software prediction of the molecular ion $\mathrm{m} / \mathrm{z}$ value and the identification of the compound by using the EI mass spectral library match using a match factor threshold of 800 . Formulas with a question mark "?" might not have been accurately identified. The ratio of the peak area of each of these questionable compounds relative to all peak areas of the detected compounds is provided in the table below

\begin{tabular}{|c|c|c|c|c|c|}
\hline Name & Formula & $\begin{array}{l}\text { Retention Times (s) on the } \\
\text { Primary, Secondary Column }\end{array}$ & $\begin{array}{l}\text { Molecular } \\
\text { Ion } \mathbf{m} / \mathbf{z}\end{array}$ & $\begin{array}{l}\text { Chromatographic } \\
\text { Peak Area }\end{array}$ & $\begin{array}{c}\text { Ratio } \\
\begin{array}{c}\text { (Peak area/total sum of } \\
\text { peak areas) }\end{array}\end{array}$ \\
\hline 3-Methylheptane & $\mathrm{C}_{8} \mathrm{H}_{18}$ & $406.002,2.402$ & 114.1403 & 169906 & \\
\hline $\begin{array}{l}\text { 1,1,3,4-Tetramethyl-cis- } \\
\text { cyclopentane }\end{array}$ & $\mathrm{C}_{9} \mathrm{H}_{18}$ & $412.003,2.448$ & 126.1403 & 32752 & \\
\hline 4-Methylpropiolactam & $\mathrm{C}_{4} \mathrm{H}_{7} \mathrm{NO}$ & $418.005,2.383$ & 85.0522 & 8125 & \\
\hline 1,1,2-Trimethylcyclopentane & $\mathrm{C}_{8} \mathrm{H}_{16}$ & $424.007,2.304$ & 112.1247 & 176745 & \\
\hline 2,4-Dimethylhexane & $\mathrm{C}_{8} \mathrm{H}_{18}$ & $442.012,2.597$ & 114.1403 & 512088 & \\
\hline 1,3-Dimethyl-cis-cyclohexane & $\mathrm{C}_{8} \mathrm{H}_{16}$ & $454.016,2.386$ & 112.1247 & 1909811 & \\
\hline 4-Methyl-1-heptanol & $\mathrm{C}_{8} \mathrm{H}_{18} \mathrm{O}$ & $454.016,2.607$ & 130.1352 & 67932 & \\
\hline 3-Methylundecane & $\mathrm{C}_{12} \mathrm{H}_{26}$ & $460.017,2.793$ & 170.2029 & 379081 & \\
\hline 1,1-Dimethylcyclohexane & $\mathrm{C}_{8} \mathrm{H}_{16}$ & $478.022,2.422$ & 112.1247 & 649319 & \\
\hline 2,6-Dimethylheptane & $\mathrm{C}_{9} \mathrm{H}_{20}$ & $478.022,2.875$ & 128.156 & 596482 & \\
\hline 1,2-Dimethylcyclohexane & $\mathrm{C}_{8} \mathrm{H}_{16}$ & $496.028,2.501$ & 112.1247 & 638086 & \\
\hline $\begin{array}{l}\text { (2S,1'S)-(-)-2-(1-Hydroxy-6- } \\
\text { methylheptyl)-2,5- } \\
\text { dihydrofuran }\end{array}$ & $\mathrm{C}_{12} \mathrm{H}_{22} \mathrm{O}_{2}$ ? & $496.028,2.693$ & 198.1614 & 83464 & 7.93E-4 \\
\hline 3,5-Dimethylheptane & $\mathrm{C}_{9} \mathrm{H}_{20}$ & $496.028,2.916$ & 128.156 & 1131183 & \\
\hline 1,4-Dimethyl-cyclohexane & $\mathrm{C}_{8} \mathrm{H}_{16}$ & $514.033,2.535$ & 112.1247 & 592032 & \\
\hline 6-Methylheptan-1-ol & $\mathrm{C}_{8} \mathrm{H}_{18} \mathrm{O}$ & $514.033,2.777$ & 130.1352 & 15811 & \\
\hline 1,3,5-Trimethylcyclohexane & $\mathrm{C}_{9} \mathrm{H}_{18}$ & $538.04,2.777$ & 126.1403 & 300219 & \\
\hline 5-Eethyldecane & $\mathrm{C}_{11} \mathrm{H}_{24}$ & $550.043,3.093$ & 156.1873 & 162299 & \\
\hline $\begin{array}{l}\text { 1,1,3-Trimethylno space } \\
\text { cyclohexane }\end{array}$ & $\mathrm{C}_{9} \mathrm{H}_{18}$ & $562.047,2.808$ & 126.1403 & 1490003 & \\
\hline 4-Methyloctane & $\mathrm{C}_{9} \mathrm{H}_{20}$ & $562.047,3.131$ & 128.156 & 678506 & \\
\hline 1,7-Dial-2-heptene & $\mathrm{C}_{7} \mathrm{H}_{10} \mathrm{O}_{2}$ & $574.05,2.664$ & 126.0675 & 17119 & \\
\hline 6-Methylundecane & $\mathrm{C}_{12} \mathrm{H}_{26}$ & $580.052,3.194$ & 170.2029 & 1235637 & \\
\hline Ethylcyclohexane & $\mathrm{C}_{8} \mathrm{H}_{16}$ & $586.053,2.685$ & 112.1247 & 501599 & \\
\hline
\end{tabular}




\begin{tabular}{|c|c|c|c|c|c|}
\hline 1,2,4-trimethylcyclohexane & $\mathrm{C}_{9} \mathrm{H}_{18}$ & $592.055,2.911$ & 126.1403 & 608681 & \\
\hline 1-Nonadecanol & $\mathrm{C}_{19} \mathrm{H}_{40} \mathrm{O} ?$ & $592.055,3.117$ & 284.3074 & 14317 & $1.36 \mathrm{E}-4$ \\
\hline Methylbenzene, & $\mathrm{C}_{7} \mathrm{H}_{8}$ & $598.057,2.057$ & 92.0621 & 104019 & \\
\hline $\begin{array}{l}\text { 1,1,3,5- } \\
\text { Tetramethylcyclohexane }\end{array}$ & $\mathrm{C}_{10} \mathrm{H}_{20}$ & $652.072,3.206$ & 140.156 & 320680 & \\
\hline Nonane & $\mathrm{C}_{9} \mathrm{H}_{20}$ & $658.074,3.389$ & 128.156 & 761784 & \\
\hline $\begin{array}{l}\text { trans-1-Ethyl-4-methyl- } \\
\text { cyclohexane }\end{array}$ & $\mathrm{C}_{9} \mathrm{H}_{18}$ & $706.088,3.096$ & 126.1403 & 1040919 & \\
\hline 3,5-Dimethyloctane & $\mathrm{C}_{10} \mathrm{H}_{22}$ & $706.088,3.682$ & 142.1716 & 1093105 & \\
\hline 2,6-Dimethyloctane, & $\mathrm{C}_{10} \mathrm{H}_{22}$ & $736.096,3.734$ & 142.1716 & 1543047 & \\
\hline 1,2-Dibutyl-cis-cyclopropane & $\mathrm{C}_{11} \mathrm{H}_{22}$ & $742.098,3.489$ & 154.1716 & 11320 & \\
\hline (Z)-5-Undecen-1-ol & $\mathrm{C}_{11} \mathrm{H}_{22} \mathrm{O}$ ? & $754.102,2.740$ & 170.1665 & 39230 & 3.72E-4 \\
\hline 3,6-Dimethyloctane & $\mathrm{C}_{10} \mathrm{H}_{22}$ & $760.103,3.770$ & 142.1716 & 432462 & \\
\hline $\begin{array}{l}\text { N-1-Methyl-6-(1'- } \\
\text { piperidinyl)hexyl-1-amine }\end{array}$ & $\mathrm{C}_{12} \mathrm{H}_{26} \mathrm{~N}_{2}$ & $766.105,3.804$ & 198.2091 & 12810 & \\
\hline 1-Ethyl-4-methylcyclohexane & $\mathrm{C}_{9} \mathrm{H}_{18}$ & $778.109,3.163$ & 126.1403 & 582755 & \\
\hline Heptanal & $\mathrm{C}_{7} \mathrm{H}_{14} \mathrm{O}$ & $790.112,3.763$ & 114.1039 & 72771 & \\
\hline 1-Ethyl-1-methylcyclohexane & $\mathrm{C}_{9} \mathrm{H}_{18}$ & $808.117,3.137$ & 126.1403 & 173691 & \\
\hline $\begin{array}{l}\text { (+)-(S)-1-Butyl-2- } \\
\text { methylenecyclopentane }\end{array}$ & $\mathrm{C}_{10} \mathrm{H}_{18}$ & $814.119,2.734$ & 138.1403 & 22288 & \\
\hline 4-Ethyloctane & $\mathrm{C}_{10} \mathrm{H}_{22}$ & $814.119,3.831$ & 142.1716 & 799473 & \\
\hline $\begin{array}{l}\text { trans-1,1,3,5- } \\
\text { Tetramethylcyclohexane }\end{array}$ & $\mathrm{C}_{10} \mathrm{H}_{20}$ & $820.121,3.410$ & 140.156 & 267160 & \\
\hline 4-Methylnonane & $\mathrm{C}_{10} \mathrm{H}_{22}$ & $832.124,3.903$ & 142.1716 & 1548636 & \\
\hline 3-Nonyne & $\mathrm{C}_{9} \mathrm{H}_{16}$ & $838.126,2.978$ & 124.1247 & 174285 & \\
\hline 3,4-Di(ethyl)-3-hexene & $\mathrm{C}_{10} \mathrm{H}_{20}$ & $838.126,3.493$ & 140.156 & 205010 & \\
\hline $\begin{array}{l}\text { 1-Azido-1- } \\
\text { ethenylcyclopropane }\end{array}$ & $\mathrm{C}_{5} \mathrm{H}_{7} \mathrm{~N}_{3}$ & $844.127,3.153$ & 109.0634 & 20279 & \\
\hline $\begin{array}{l}\text { 1,2-Diethyl-3- } \\
\text { methylcyclohexane }\end{array}$ & $\mathrm{C}_{11} \mathrm{H}_{22}$ & $850.129,3.494$ & 154.1716 & 378334 & \\
\hline Propylcyclohexane & $\mathrm{C}_{9} \mathrm{H}_{18}$ & $856.131,3.275$ & 126.1403 & 338322 & \\
\hline 3-Methylnonane & $\mathrm{C}_{10} \mathrm{H}_{22}$ & $868.134,3.924$ & 142.1716 & 749919 & \\
\hline cis-Octahydro- $1 \mathrm{H}$-indene & $\mathrm{C}_{9} \mathrm{H}_{16}$ & $874.136,3.082$ & 124.1247 & 119334 & \\
\hline 2,3,3-Trimethyl-(Z)- 4-nonene & $\mathrm{C}_{12} \mathrm{H}_{24}$ & $874.136,3.538$ & 168.1873 & 174139 & \\
\hline $\begin{array}{l}\text { 1,7,7-Trimethyl- } \\
\text { bicyclo[2.2.1]heptane }\end{array}$ & $\mathrm{C}_{10} \mathrm{H}_{18}$ & $886.14,2.975$ & 138.1403 & 9472 & \\
\hline 1,2-Dimethylbenzene & $\mathrm{C}_{8} \mathrm{H}_{10}$ & $904.145,2.407$ & 106.0777 & 163761 & \\
\hline
\end{tabular}




\begin{tabular}{|c|c|c|c|c|c|}
\hline 3-Methoxy-1-propyne & $\mathrm{C}_{4} \mathrm{H}_{6} \mathrm{O}$ & $910.146,3.478$ & 70.0413 & 24350 & \\
\hline 2,6,10-Trimethyltridecane & $\mathrm{C}_{16} \mathrm{H}_{34}$ & $916.148,4.257$ & 226.2655 & 91976 & \\
\hline $\begin{array}{l}\text { 1-Ethyl-trans-1,3- } \\
\text { dimethylcyclohexane }\end{array}$ & $\mathrm{C}_{10} \mathrm{H}_{20}$ & $922.15,3.559$ & 140.156 & 621883 & \\
\hline $\begin{array}{l}\text { 1,1,2,3- } \\
\text { Tetramethylcyclohexane }\end{array}$ & $\mathrm{C}_{10} \mathrm{H}_{20}$ & $940.155,3.461$ & 140.156 & 202761 & \\
\hline 4-Methylundecane & $\mathrm{C}_{12} \mathrm{H}_{26}$ & $946.157,4.238$ & 170.2029 & 54013 & \\
\hline Di-N-methylpentan-2,3-imine & $\mathrm{C}_{7} \mathrm{H}_{14} \mathrm{~N}_{2}$ & $952.158,3.398$ & 126.1151 & 5961 & \\
\hline $\begin{array}{l}\text { 1-Ethyl-1,3-dimethyl-cis- } \\
\text { cyclohexane }\end{array}$ & $\mathrm{C}_{10} \mathrm{H}_{20}$ & $952.158,3.518$ & 140.156 & 266473 & \\
\hline $\begin{array}{l}\text { 3,7,7-Trimethyl-1,3,8- } \\
\text { nonatrien-5-one }\end{array}$ & $\mathrm{C}_{12} \mathrm{H}_{18} \mathrm{O}$ ? & $952.158,3.565$ & 178.1352 & 15848 & $1.50 \mathrm{E}-4$ \\
\hline $\begin{array}{l}\text { 2,2-Dimethyl-3,5-diphenyl-, 1- } \\
\text { oxide-2H-pyrrole }\end{array}$ & $\mathrm{C}_{18} \mathrm{H}_{17} \mathrm{NO} ?$ & $952.158,3.769$ & 263.1305 & 15138 & $1.43 \mathrm{E}-4$ \\
\hline Decane & $\mathrm{C}_{10} \mathrm{H}_{22}$ & $976.165,4.063$ & 142.1716 & 1985109 & \\
\hline $\begin{array}{l}\text { 1-Isopropyl-4- } \\
\text { methylcyclohexane }\end{array}$ & $\mathrm{C}_{10} \mathrm{H}_{20}$ & $982.167,3.574$ & 140.156 & 325676 & \\
\hline 2,4-Dimethyldecane & $\mathrm{C}_{12} \mathrm{H}_{26}$ & $994.171,4.294$ & 170.2029 & 435063 & \\
\hline $\begin{array}{l}\text { 1-Methyl-3-propyl- } \\
\text { cyclohexane }\end{array}$ & $\mathrm{C}_{10} \mathrm{H}_{20}$ & $1006.17,3.657$ & 140.156 & 505881 & \\
\hline $\begin{array}{l}\text { trans-1-Methyl-4-(1- } \\
\text { methylethyl)cyclohexane }\end{array}$ & $\mathrm{C}_{10} \mathrm{H}_{20}$ & $1018.18,3.662$ & 140.156 & 340150 & \\
\hline 1,4-Dimethylbenzene & $\mathrm{C}_{8} \mathrm{H}_{10}$ & $1024.18,2.427$ & 106.0777 & 73255 & \\
\hline 2,6-Dimethylnonane & $\mathrm{C}_{11} \mathrm{H}_{24}$ & $1024.18,4.351$ & 156.1873 & 1774353 & \\
\hline $\begin{array}{l}\text { 1,5-Diethyl-2,3-dimethyl- } \\
\text { cyclohexane }\end{array}$ & $\mathrm{C}_{12} \mathrm{H}_{24}$ & $1054.19,3.885$ & 168.1873 & 99866 & \\
\hline 6-Ethyl-2-methyloctane & $\mathrm{C}_{11} \mathrm{H}_{24}$ & $1054.19,4.330$ & 156.1873 & 503297 & \\
\hline 1-(2-Propynyloxy)-2-butene & $\mathrm{C}_{7} \mathrm{H}_{10} \mathrm{O}$ & $1072.19,3.387$ & 110.0726 & 42115 & \\
\hline Methyl benzenesulphinate & $\mathrm{C}_{18} \mathrm{H}_{28} \mathrm{O}_{2} \mathrm{~S} ?$ & $1078.19,3.859$ & 308.1805 & 44289 & $4.20 \mathrm{E}-4$ \\
\hline $\begin{array}{l}\text { 2,4-Di(dimethylethyl)-2- } \\
\text { methyl-2,5-dihydro-1H- } \\
\text { imidazole }\end{array}$ & $\mathrm{C}_{12} \mathrm{H}_{24} \mathrm{~N}_{2}$ ? & $1078.19,3.923$ & 196.1934 & 17418 & $1.65 \mathrm{E}-4$ \\
\hline 2,9-Dimethyldecane & $\mathrm{C}_{12} \mathrm{H}_{26}$ & $1078.19,4.366$ & 170.2029 & 565415 & \\
\hline $\begin{array}{l}\text { (1S,2S)-1-Amino-2- } \\
\text { propylcyclopropanecarbonitrile }\end{array}$ & $\mathrm{C}_{7} \mathrm{H}_{12} \mathrm{~N}_{2}$ & $1090.2,3.427$ & 124.0995 & 23082 & \\
\hline $\begin{array}{l}\text { 1-Methyl-4-(1-methylethyl)- } \\
\text { cyclohexane }\end{array}$ & $\mathrm{C}_{10} \mathrm{H}_{20}$ & $1096.2,3.631$ & 140.156 & 231973 & \\
\hline $\begin{array}{l}\text { 2-Ethyl-4-methyl-1H- } \\
\text { imidazole }\end{array}$ & $\mathrm{C}_{6} \mathrm{H}_{10} \mathrm{~N}_{2}$ & $1096.2,3.785$ & 110.0838 & 20016 & \\
\hline 3,7-Dimethylnonane & $\mathrm{C}_{11} \mathrm{H}_{24}$ & $1102.2,4.335$ & 156.1873 & 475618 & \\
\hline $\begin{array}{l}\text { 5,6-Epoxy-6-methyl-1,9- } \\
\text { decadien-4-one }\end{array}$ & $\mathrm{C}_{11} \mathrm{H}_{16} \mathrm{O}_{2}$ ? & $1126.21,3.917$ & 180.1145 & 64743 & $6.15 \mathrm{E}-4$ \\
\hline $\begin{array}{l}\text { rac-(+-)-2-(tert- } \\
\text { Butylsulfonyl)pentyl acetate }\end{array}$ & $\mathrm{C}_{11} \mathrm{H}_{22} \mathrm{O}_{4} \mathrm{~S}$ & $1126.21,4.324$ & 250.1233 & 14237 & \\
\hline 2-Methylundecane & $\mathrm{C}_{12} \mathrm{H}_{26}$ & $1138.21,4.690$ & 170.2029 & 30465 & \\
\hline
\end{tabular}




\begin{tabular}{|c|c|c|c|c|c|}
\hline 6-Ethyltridecane & $\mathrm{C}_{14} \mathrm{H}_{30}$ & $1144.21,4.377$ & 198.2342 & 379146 & \\
\hline 2-methylnonane & $\mathrm{C}_{10} \mathrm{H}_{22}$ & $1168.22,4.361$ & 142.1716 & 105947 & \\
\hline $\begin{array}{l}\text { 2,6,6-Trimethyl } \\
\text { bicyclo[3.1.1]heptane }\end{array}$ & $\mathrm{C}_{10} \mathrm{H}_{18}$ & $1180.22,3.640$ & 138.1403 & 20311 & \\
\hline 2,2-Dimethylundecane & $\mathrm{C}_{13} \mathrm{H}_{28}$ & $1180.22,4.709$ & 184.2186 & 41243 & \\
\hline 4-Methyldecane & $\mathrm{C}_{11} \mathrm{H}_{24}$ & $1192.23,4.384$ & 156.1873 & 509049 & \\
\hline 2-Methyldecane & $\mathrm{C}_{11} \mathrm{H}_{24}$ & $1198.23,4.433$ & 156.1873 & 610534 & \\
\hline Butylcyclohexane & $\mathrm{C}_{10} \mathrm{H}_{20}$ & $1234.24,3.682$ & 140.156 & 404759 & \\
\hline 3-Methyldecane & $\mathrm{C}_{11} \mathrm{H}_{24}$ & $1234.24,4.413$ & 156.1873 & 554663 & \\
\hline Ethylcyclooctane & $\mathrm{C}_{10} \mathrm{H}_{20}$ & $1240.24,3.929$ & 140.156 & 169894 & \\
\hline $\begin{array}{l}\text { 4,9-Dimethyl-7- } \\
\text { oxabicyclo[4.3.0]nonan-8-one }\end{array}$ & $\mathrm{C}_{10} \mathrm{H}_{16} \mathrm{O}_{2} ?$ & $1246.24,3.688$ & 168.1145 & 14545 & $1.38 \mathrm{E}-4$ \\
\hline 2,2,5-Trimethylhexane & $\mathrm{C}_{9} \mathrm{H}_{20}$ & $1258.25,4.673$ & 128.156 & 16313 & \\
\hline (1-Methylethyl)benzene & $\mathrm{C}_{9} \mathrm{H}_{12}$ & $1264.25,2.623$ & 120.0934 & 64517 & \\
\hline 3,3,6-Trimethyl-1,2-dioxane & $\mathrm{C}_{7} \mathrm{H}_{13} \mathrm{O}_{2}$ & $1264.25,3.932$ & 129.091 & 36607 & \\
\hline $\begin{array}{l}\text { 1-Isopropyl-2- } \\
\text { ((phenylthio)methyl)cyclopent } \\
\text { ene }\end{array}$ & $\mathrm{C}_{15} \mathrm{H}_{20} \mathrm{~S}$ & $1276.25,3.615$ & 232.128 & 9355 & \\
\hline $\begin{array}{l}\text { Tetrahydro-2- } \\
\text { furanpropanenitrile }\end{array}$ & $\mathrm{C}_{7} \mathrm{H}_{11} \mathrm{NO}$ & $1276.25,4.694$ & 125.0835 & 21980 & \\
\hline Mesitylene & $\mathrm{C}_{9} \mathrm{H}_{12}$ & $1288.25,2.654$ & 120.0934 & 397011 & \\
\hline $\begin{array}{l}\text { 1-Methyl-2-(2'-methylprop-2'- } \\
\text { enyl)cyclododecan-1-ol }\end{array}$ & $\mathrm{C}_{17} \mathrm{H}_{32} \mathrm{O}$ ? & $1300.26,3.901$ & 252.2448 & 26671 & $2.53 \mathrm{E}-4$ \\
\hline $\begin{array}{l}\text { 4,4,7,7-Tetramethyl-1,9- } \\
\text { decadiene }\end{array}$ & $\mathrm{C}_{14} \mathrm{H}_{26}$ & $1312.26,4.277$ & 194.2029 & 105641 & \\
\hline $\begin{array}{l}\text { 2,2,5-Trimethyl-(S)-6-hepten- } \\
\text { 1-ol }\end{array}$ & $\mathrm{C}_{10} \mathrm{H}_{20} \mathrm{O} ?$ & $1318.26,3.927$ & 156.1509 & 57926 & $5.50 \mathrm{E}-4$ \\
\hline Cacospongin A & $\mathrm{C}_{20} \mathrm{H}_{30} \mathrm{O} ?$ & $1330.27,3.622$ & 286.2291 & 21494 & $2.04 \mathrm{E}-4$ \\
\hline 2-Methyl-4-hexyn-3-ol & $\mathrm{C}_{7} \mathrm{H}_{12} \mathrm{O}$ & $1330.27,4.356$ & 112.0883 & 85574 & \\
\hline $\begin{array}{l}\text { 2,2,4,5-Tetramethylhex-5-en- } \\
\text { 3-one }\end{array}$ & $\mathrm{C}_{10} \mathrm{H}_{14} \mathrm{O} ?$ & $1342.27,4.691$ & 150.1039 & 62765 & $5.96 \mathrm{E}-4$ \\
\hline Octacosane & $\mathrm{C}_{28} \mathrm{H}_{58}$ & $1348.27,4.651$ & 394.4533 & 129622 & \\
\hline Dodecane & $\mathrm{C}_{12} \mathrm{H}_{26}$ & $1366.28,4.413$ & 170.2029 & 4046704 & \\
\hline $\begin{array}{l}\text { 4-(1- } \\
\text { Methylethylidene)bicyclo[3.1.0 } \\
\text { ]hex-2-ene }\end{array}$ & $\mathrm{C}_{9} \mathrm{H}_{12}$ & $1372.28,2.602$ & 120.0934 & 18414 & \\
\hline Dotriacontane & $\mathrm{C}_{32} \mathrm{H}_{66}$ & $1372.28,4.660$ & 450.5159 & 49812 & \\
\hline $\begin{array}{l}\text { cis-1-Ethyl-4-methyl } \\
\text { cyclohexane }\end{array}$ & $\mathrm{C}_{9} \mathrm{H}_{18}$ & $1384.28,3.902$ & 126.1403 & 127255 & \\
\hline $1,2,4,6$-Tetraeneheptane & $\mathrm{C}_{7} \mathrm{H}_{8}$ & $1402.29,2.828$ & 92.0621 & 11088 & \\
\hline
\end{tabular}




\begin{tabular}{|c|c|c|c|c|c|}
\hline 1,2,4-Trimethylbenzene & $\mathrm{C}_{9} \mathrm{H}_{12}$ & $1414.29,2.664$ & 120.0934 & 292327 & \\
\hline 2,4,6,8-Tetramethylundecene & $\mathrm{C}_{15} \mathrm{H}_{30}$ & $1414.29,4.227$ & 210.2342 & 137781 & \\
\hline 3,9-Epoxy-p-menthan-9-one & $\mathrm{C}_{10} \mathrm{H}_{16} \mathrm{O}_{2}$ ? & $1420.29,3.535$ & 168.1145 & 28855 & $2.74 \mathrm{E}-4$ \\
\hline 3,3-Dimethylhexane & $\mathrm{C}_{8} \mathrm{H}_{18}$ & $1420.29,4.675$ & 114.1403 & 432137 & \\
\hline $\begin{array}{l}\text { Bis(2-cyclohexyl-1- } \\
\text { methylethyl)-1,2- } \\
\text { dioxidediazene }\end{array}$ & $\mathrm{C}_{18} \mathrm{H}_{34} \mathrm{~N}_{2} \mathrm{O}_{2}$ & $1426.29,3.776$ & 310.2615 & 32449 & $3.08 \mathrm{E}-4$ \\
\hline 1-Ethyl-2-propylcyclohexane & $\mathrm{C}_{11} \mathrm{H}_{22}$ & $1438.3,3.945$ & 154.1716 & 297536 & \\
\hline 3,7-Dimethyldecane & $\mathrm{C}_{12} \mathrm{H}_{26}$ & $1444.3,4.644$ & 170.2029 & 768556 & \\
\hline Decahydronaphthalene & $\mathrm{C}_{10} \mathrm{H}_{18}$ & $1468.31,3.343$ & 138.1403 & 151938 & \\
\hline $\begin{array}{l}\text { 2-Methyl-4-(2- } \\
\text { methylpropyl)cyclopentanone }\end{array}$ & $\mathrm{C}_{10} \mathrm{H}_{18} \mathrm{O}$ ? & $1468.31,3.934$ & 154.1352 & 447746 & $4.25 \mathrm{E}-3$ \\
\hline Tetradecane & $\mathrm{C}_{14} \mathrm{H}_{30}$ & $1468.31,4.644$ & 198.2342 & 329958 & \\
\hline Isobutylbenzene & $\mathrm{C}_{10} \mathrm{H}_{14}$ & $1492.31,2.768$ & 134.109 & 51406 & \\
\hline 3,3-Dimethyl-1-nonyne & $\mathrm{C}_{11} \mathrm{H}_{20}$ & $1522.32,3.493$ & 152.156 & 4336 & \\
\hline 7-Methylpentadecane & $\mathrm{C}_{16} \mathrm{H}_{34}$ & $1528.32,4.594$ & 226.2655 & 279199 & \\
\hline Octadecane & $\mathrm{C}_{18} \mathrm{H}_{38}$ & $1540.33,4.892$ & 254.2968 & 31246 & \\
\hline 3-Ethoxy-1-propyne & $\mathrm{C}_{5} \mathrm{H}_{8} \mathrm{O}$ & $1552.33,4.136$ & 84.057 & 137533 & \\
\hline 1,2,3-Trimethylbenzene & $\mathrm{C}_{9} \mathrm{H}_{12}$ & $1588.34,2.613$ & 120.0934 & 67729 & \\
\hline $\begin{array}{l}\text { 1-Methyl-3-(1-methylethyl)- } \\
\text { cyclopentane }\end{array}$ & $\mathrm{C}_{9} \mathrm{H}_{18}$ & $1594.34,3.817$ & 126.1403 & 22942 & \\
\hline 2,5,9-Trimethyldecane & $\mathrm{C}_{13} \mathrm{H}_{28}$ & $1606.35,4.922$ & 184.2186 & 479568 & \\
\hline (E)-2,3-Epoxytetradecan-1-ol & $\mathrm{C}_{14} \mathrm{H}_{28} \mathrm{O}_{2}$ ? & $1630.35,4.184$ & 228.2084 & 70100 & $6.66 \mathrm{E}-4$ \\
\hline (1-Methylpropyl)benzene & $\mathrm{C}_{10} \mathrm{H}_{14}$ & $1636.35,2.806$ & 134.109 & 23460 & \\
\hline Pentyl-cyclohexane & $\mathrm{C}_{11} \mathrm{H}_{22}$ & $1654.36,3.873$ & 154.1716 & 502385 & \\
\hline $\begin{array}{l}\text { Decahydro-2- } \\
\text { methylnaphthalene }\end{array}$ & $\mathrm{C}_{11} \mathrm{H}_{20}$ & $1660.36,3.543$ & 152.156 & 106838 & \\
\hline $\begin{array}{l}\text { Ethyl 5-(1- } \\
\text { dimethylaminoethylidene)cycl } \\
\text { openta-1,3- } \\
\text { dienyl]hydroxyacetate }\end{array}$ & $\mathrm{C}_{13} \mathrm{H}_{19} \mathrm{NO}_{3} ?$ & $1666.36,4.785$ & 237.1359 & 29568 & $2.80 \mathrm{E}-4$ \\
\hline 4-Ethyl-1,2-dimethylbenzene & $\mathrm{C}_{10} \mathrm{H}_{14}$ & $1672.37,2.757$ & 134.109 & 223735 & \\
\hline $\begin{array}{l}\text { 1,2-di[p-Methylbenzoyloxy-(p- } \\
\text { tolyl)]ethene }\end{array}$ & $\mathrm{C}_{32} \mathrm{H}_{28} \mathrm{O}_{4}$ & $1678.37,2.744$ & 476.1982 & 9764 & \\
\hline 1,3-Dimethyladamantane & $\mathrm{C}_{12} \mathrm{H}_{20}$ & $1690.37,3.525$ & 164.156 & 50328 & \\
\hline $\begin{array}{l}\text { 1-Isobutyl-2,5- } \\
\text { dimethylcyclohexane }\end{array}$ & $\mathrm{C}_{12} \mathrm{H}_{24}$ & $1690.37,4.150$ & 168.1873 & 406912 & \\
\hline $\begin{array}{l}\text { 1-Methylimidazole-5- } \\
\text { carbaldehyde }\end{array}$ & $\mathrm{C}_{5} \mathrm{H}_{6} \mathrm{~N}_{2} \mathrm{O}$ & $1696.37,4.164$ & 110.0475 & 16321 & \\
\hline
\end{tabular}




\begin{tabular}{|c|c|c|c|c|c|}
\hline 2-(1-Phenylethyl)phenol & $\mathrm{C}_{14} \mathrm{H}_{14} \mathrm{O}$ ? & $1702.37,4.755$ & 198.1039 & 25134 & $2.38 \mathrm{E}-4$ \\
\hline 1,17-Octadecadiene & $\mathrm{C}_{18} \mathrm{H}_{34}$ & $1720.38,4.061$ & 250.2655 & 53775 & \\
\hline $\begin{array}{l}\text { 1- } \\
\text { Methyldecahydronaphthalene }\end{array}$ & $\mathrm{C}_{11} \mathrm{H}_{20}$ & $1756.39,3.489$ & 152.156 & 68107 & \\
\hline Dioctyl disulfide & $\mathrm{C}_{16} \mathrm{H}_{34} \mathrm{~S}_{2}$ & $1762.39,4.731$ & 290.2096 & 33028 & \\
\hline 1-Ethyl-2,3-dimethylbenzene & $\mathrm{C}_{10} \mathrm{H}_{14}$ & $1768.39,2.748$ & 134.109 & 33328 & \\
\hline $\begin{array}{l}\text { cis-1-Butyl-2- } \\
\text { undecylcyclopropane }\end{array}$ & $\mathrm{C}_{18} \mathrm{H}_{36}$ & $1768.39,4.391$ & 252.2812 & 57743 & \\
\hline Pentadecane & $\mathrm{C}_{15} \mathrm{H}_{32}$ & $1768.39,4.752$ & 212.2499 & 161040 & \\
\hline Tridecane & $\mathrm{C}_{13} \mathrm{H}_{28}$ & $1774.39,4.536$ & 184.2186 & 6196757 & \\
\hline 2,6-Dimethylundecane & $\mathrm{C}_{13} \mathrm{H}_{28}$ & $1786.4,4.798$ & 184.2186 & 5345893 & \\
\hline (1-Methylbutyl)benzene & $\mathrm{C}_{11} \mathrm{H}_{16}$ & $1792.4,2.942$ & 148.1247 & 80220 & \\
\hline $\begin{array}{l}\text { 3-Acetyl-3- } \\
\text { methylcyclohexanone }\end{array}$ & $\mathrm{C}_{9} \mathrm{H}_{14} \mathrm{O}_{2}$ & $1792.4,4.316$ & 154.0988 & 31137 & \\
\hline $\begin{array}{l}\text { 1-Methyl-3-propyl- } \\
\text { cyclooctane }\end{array}$ & $\mathrm{C}_{12} \mathrm{H}_{24}$ & $1810.4,4.132$ & 168.1873 & 69988 & \\
\hline $\begin{array}{l}\text { 1-Methyl-4-(1-methylethyl)- } \\
\text { benzene }\end{array}$ & $\mathrm{C}_{10} \mathrm{H}_{14}$ & $1822.41,2.904$ & 134.109 & 57972 & \\
\hline 3-Phenyl-2-butanone & $\mathrm{C}_{10} \mathrm{H}_{12} \mathrm{O}$ & $1822.41,2.946$ & 148.0883 & 46989 & \\
\hline $\begin{array}{l}\text { 1-Methyl-2-pentyl- } \\
\text { cyclohexane }\end{array}$ & $\mathrm{C}_{12} \mathrm{H}_{24}$ & $1822.41,4.094$ & 168.1873 & 618065 & \\
\hline 2,6,10-Trimethyldodecane & $\mathrm{C}_{15} \mathrm{H}_{32}$ & $1834.41,4.731$ & 212.2499 & 1301290 & \\
\hline $\begin{array}{l}\text { (4-Methylphenyl)propanoic } \\
\text { acid }\end{array}$ & $\mathrm{C}_{10} \mathrm{H}_{12} \mathrm{O}_{2}$ ? & $1852.42,2.905$ & 164.0832 & 24368 & 2.31E-4 \\
\hline $\begin{array}{l}\text { 1,1'-(1,2-Dimethyl-1,2- } \\
\text { ethanediyl)bis-cyclohexane }\end{array}$ & $\mathrm{C}_{16} \mathrm{H}_{30}$ & $1852.42,4.063$ & 222.2342 & 180013 & \\
\hline $\begin{array}{l}\text { Decahydro-2,2- } \\
\text { dimethylnaphthalene }\end{array}$ & $\mathrm{C}_{12} \mathrm{H}_{22}$ & $1858.42,3.692$ & 166.1716 & 66059 & \\
\hline $\begin{array}{l}\text { Methylcyclohexane-methyl } \\
\text { amine }\end{array}$ & $\mathrm{C}_{8} \mathrm{H}_{17} \mathrm{~N}$ & $1864.42,4.288$ & 127.1356 & 24547 & \\
\hline 2,2-Dimethyl-3-butenal & $\mathrm{C}_{6} \mathrm{H}_{10} \mathrm{O}$ & $1870.42,4.060$ & 98.0726 & 30717 & \\
\hline 3-Phenylbutanal & $\mathrm{C}_{10} \mathrm{H}_{12} \mathrm{O}$ ? & $1882.43,2.897$ & 148.0883 & 13009 & $1.23 \mathrm{E}-4$ \\
\hline (S)-Undec-1-en-4-ol & $\mathrm{C}_{11} \mathrm{H}_{22} \mathrm{O}$ ? & $1882.43,4.048$ & 170.1665 & 22126 & $2.10 \mathrm{E}-4$ \\
\hline 3,4-Dimethylcumene & $\mathrm{C}_{11} \mathrm{H}_{16}$ & $1888.43,2.854$ & 148.1247 & 60767 & \\
\hline (1-Methylpropyl)-cyclohexane & $\mathrm{C}_{10} \mathrm{H}_{20}$ & $1900.43,4.027$ & 140.156 & 182248 & \\
\hline 5,5-Dimethylfuran-2(5H)-one & $\mathrm{C}_{6} \mathrm{H}_{8} \mathrm{O}_{2}$ & $1936.44,4.338$ & 112.0519 & 37964 & \\
\hline 2,7,10-Trimethyldodecane & $\mathrm{C}_{15} \mathrm{H}_{32}$ & $1936.44,4.963$ & 212.2499 & 326229 & \\
\hline (3-Methylpentyl)cyclohexane & $\mathrm{C}_{12} \mathrm{H}_{24}$ & $1942.44,3.975$ & 168.1873 & 141843 & \\
\hline 3-Methyldodecane & $\mathrm{C}_{13} \mathrm{H}_{28}$ & $1948.44,4.968$ & 184.2186 & 72526 & \\
\hline
\end{tabular}




\begin{tabular}{|c|c|c|c|c|}
\hline 4-Ethylundecane & $\mathrm{C}_{13} \mathrm{H}_{28}$ & $1954.45,4.654$ & 184.2186 & 198420 \\
\hline $\begin{array}{l}\text { 1-Methyl-2-(1- } \\
\text { methylethyl)benzene }\end{array}$ & $\mathrm{C}_{10} \mathrm{H}_{14}$ & $1960.45,2.751$ & 134.109 & 86445 \\
\hline $\begin{array}{l}\text { 1-Ethyl-cis-1,4-dimethyl } \\
\text { cyclohexane }\end{array}$ & $\mathrm{C}_{10} \mathrm{H}_{20}$ & $1960.45,4.225$ & 140.156 & 146673 \\
\hline 6-Methyldodecane & $\mathrm{C}_{13} \mathrm{H}_{28}$ & $1972.45,4.649$ & 184.2186 & 1593030 \\
\hline $\begin{array}{l}\text { 3-Ethyl-2,3-dihydro-1- } \\
\text { benzofuran }\end{array}$ & $\mathrm{C}_{10} \mathrm{H}_{12} \mathrm{O}$ & $1984.45,2.868$ & 148.0883 & 14147 \\
\hline $\begin{array}{l}\text { (1R,3S)-7,7-Dimethyl-2- } \\
\text { oxabicyclo[4.1.1.]octan-3-one }\end{array}$ & $\mathrm{C}_{9} \mathrm{H}_{14} \mathrm{O}_{2}$ & $1984.45,4.289$ & 154.0988 & 125028 \\
\hline Tricosane & $\mathrm{C}_{23} \mathrm{H}_{48}$ & $1990.46,4.913$ & 324.3751 & 16514 \\
\hline $1,2,3,4$-Tetramethylbenzene & $\mathrm{C}_{10} \mathrm{H}_{14}$ & $1996.46,2.715$ & 134.109 & 139120 \\
\hline Germacrane & $\mathrm{C}_{15} \mathrm{H}_{30}$ & $2002.46,4.224$ & 210.2342 & 250312 \\
\hline 4-Methyldodecane & $\mathrm{C}_{13} \mathrm{H}_{28}$ & $2002.46,4.639$ & 184.2186 & 497826 \\
\hline 2,3,7-Trimethyloctane & $\mathrm{C}_{11} \mathrm{H}_{24}$ & $2002.46,4.953$ & 156.1873 & 4067569 \\
\hline 1,2-Diethylcyclooctane & $\mathrm{C}_{12} \mathrm{H}_{24}$ & $2014.46,4.257$ & 168.1873 & 119716 \\
\hline 2-Methyl-dodecane & $\mathrm{C}_{13} \mathrm{H}_{28}$ & $2014.46,4.665$ & 184.2186 & 995367 \\
\hline (1,1-Dimethylpropyl)benzene & $\mathrm{C}_{11} \mathrm{H}_{16}$ & $2020.47,2.865$ & 148.1247 & 133266 \\
\hline 1-Pentylcyclohexene & $\mathrm{C}_{11} \mathrm{H}_{20}$ & $2026.47,3.342$ & 152.156 & 32422 \\
\hline $\begin{array}{l}\text { 1-Ethyl-4-(1-methylethyl)- } \\
\text { benzene }\end{array}$ & $\mathrm{C}_{11} \mathrm{H}_{16}$ & $2038.47,2.823$ & 148.1247 & 34803 \\
\hline $\begin{array}{l}\text { N-(But-2-enoyl)prop-2- } \\
\text { enamide }\end{array}$ & $\mathrm{C}_{7} \mathrm{H}_{11} \mathrm{NO}$ & $2038.47,4.512$ & 125.0835 & 48841 \\
\hline 4-Methyltridecane & $\mathrm{C}_{14} \mathrm{H}_{30}$ & $2038.47,4.575$ & 198.2342 & 106789 \\
\hline $\begin{array}{l}\text { Methyl p-(isopropyl)benzyl } \\
\text { sulfoxide }\end{array}$ & $\mathrm{C}_{11} \mathrm{H}_{16} \mathrm{OS}$ & $2044.47,2.805$ & 196.0916 & 10490 \\
\hline $\begin{array}{l}\text { 1-Methyl-4-(1-methylbutyl)- } \\
\text { cyclohexane }\end{array}$ & $\mathrm{C}_{12} \mathrm{H}_{24}$ & $2062.48,4.207$ & 168.1873 & 286511 \\
\hline 2-Methyladamantane & $\mathrm{C}_{11} \mathrm{H}_{18}$ & $2074.48,3.106$ & 150.1403 & 31306 \\
\hline Hexylcyclohexane & $\mathrm{C}_{12} \mathrm{H}_{24}$ & $2086.48,3.919$ & 168.1873 & 658005 \\
\hline Undecane & $\mathrm{C}_{11} \mathrm{H}_{24}$ & $2092.49,4.784$ & 156.1873 & 107421 \\
\hline Hexyl butyl ether & $\mathrm{C}_{10} \mathrm{H}_{22} \mathrm{O}$ & $2116.49,4.808$ & 158.1665 & 56223 \\
\hline $\begin{array}{l}\text { 2-(3,5-Dimethyl-1-adamantyl)- } \\
\text { 1,2-dicyanoethane }\end{array}$ & $\mathrm{C}_{16} \mathrm{H}_{22} \mathrm{~N}_{2}$ & $2128.5,3.554$ & 242.1778 & 27424 \\
\hline $\begin{array}{l}\text { [[2-(2-Propenyloxy)-1- } \\
\text { propenyl]sulfonyl]-(E)- } \\
\text { benzene }\end{array}$ & $\mathrm{C}_{12} \mathrm{H}_{14} \mathrm{O}_{3} \mathrm{~S}$ & $2134.5,4.107$ & 238.0658 & 48790 \\
\hline $\begin{array}{l}\text { 8-Methyl-1- } \\
{[(\text { phenylthio)methyl]cycloocte }} \\
\text { ne }\end{array}$ & $\mathrm{C}_{16} \mathrm{H}_{22} \mathrm{~S}$ & $2152.5,3.582$ & 246.1437 & 16563 \\
\hline $\begin{array}{l}\text { 1-Isopropyl-1-methyl- } \\
\text { cyclohexane }\end{array}$ & $\mathrm{C}_{10} \mathrm{H}_{20}$ & $2152.5,4.093$ & 140.156 & 125215 \\
\hline
\end{tabular}




\begin{tabular}{|c|c|c|c|c|c|}
\hline $\begin{array}{l}\text { 1-Propanoyl-2- } \\
\text { (butanoyl)ethylene }\end{array}$ & $\mathrm{C}_{9} \mathrm{H}_{14} \mathrm{O}_{2} ?$ & $2164.51,4.026$ & 154.0988 & 16824 & $1.17 \mathrm{E}-4$ \\
\hline $\begin{array}{l}7,7- \\
\text { Dimethylbicyclo[3.2.1]octan- } \\
\text { 1-ol }\end{array}$ & $\mathrm{C}_{10} \mathrm{H}_{18} \mathrm{O}$ & $2164.51,4.109$ & 154.1352 & 12387 & \\
\hline 2,2-Dimethylheptanenitrile & $\mathrm{C}_{9} \mathrm{H}_{17} \mathrm{~N}$ & $2164.51,4.370$ & 139.1356 & 33297 & \\
\hline $\begin{array}{l}\text { 1,4-Dimethyl-2-(2- } \\
\text { methylpropyl)benzene }\end{array}$ & $\mathrm{C}_{12} \mathrm{H}_{18}$ & $2170.51,2.978$ & 162.1403 & 80472 & \\
\hline 3-Methyl-1-hexen-5-one & $\mathrm{C}_{7} \mathrm{H}_{12} \mathrm{O}$ & $2194.51,4.467$ & 112.0883 & 34875 & \\
\hline $\begin{array}{l}\text { 2,3-Dihydro-1-methyl-1H- } \\
\text { Indene }\end{array}$ & $\mathrm{C}_{10} \mathrm{H}_{12}$ & $2230.53,2.545$ & 132.0934 & 9772 & \\
\hline $\begin{array}{l}\text { 1-Ethyl-3-methyl- } \\
\text { cyclopentane }\end{array}$ & $\mathrm{C}_{8} \mathrm{H}_{16}$ & $2236.53,4.200$ & 112.1247 & 57632 & \\
\hline $\begin{array}{l}\text { (E)-5-Methyl-7-(2',6',6'- } \\
\text { trimethylcyclohex-1'- } \\
\text { enyl)hept-4-en-2-one } \\
\end{array}$ & $\mathrm{C}_{17} \mathrm{H}_{28} \mathrm{O}$ ? & $2242.53,3.741$ & 248.2135 & 17947 & $1.70 \mathrm{E}-4$ \\
\hline Heptadecane & $\mathrm{C}_{17} \mathrm{H}_{36}$ & $2248.53,4.701$ & 240.2812 & 202430 & \\
\hline 6,9-Dimethyltetradecane & $\mathrm{C}_{16} \mathrm{H}_{34}$ & $2260.53,4.680$ & 226.2655 & 213691 & \\
\hline $\begin{array}{l}\text { (exo)-9- } \\
\text { Methylbicyclo[6.1.0]nonane }\end{array}$ & $\mathrm{C}_{10} \mathrm{H}_{18}$ & $2302.55,3.745$ & 138.1403 & 9085 & \\
\hline $\begin{array}{l}\text { 1-(1,1-Dimethylethyl)-4- } \\
\text { methylbenzene }\end{array}$ & $\mathrm{C}_{11} \mathrm{H}_{16}$ & $2308.55,2.766$ & 148.1247 & 46560 & \\
\hline $\begin{array}{l}\text { 1-Ethyl-1,4-dimethyl-trans- } \\
\text { cyclohexane }\end{array}$ & $\mathrm{C}_{10} \mathrm{H}_{20}$ & $2314.55,4.270$ & 140.156 & 71787 & \\
\hline 2,2-Dimethylindan & $\mathrm{C}_{11} \mathrm{H}_{14}$ & $2320.55,2.676$ & 146.109 & 12796 & \\
\hline $\begin{array}{l}\text { 6-Methyl-1,2,3,4- } \\
\text { tetrahydronaphthalene-1- } \\
\text { methanol }\end{array}$ & $\mathrm{C}_{12} \mathrm{H}_{16} \mathrm{O}$ ? & $2320.55,2.800$ & 176.1196 & 21369 & $1.49 \mathrm{E}-4$ \\
\hline Hexadecane & $\mathrm{C}_{16} \mathrm{H}_{34}$ & $2320.55,4.634$ & 226.2655 & 279836 & \\
\hline $\begin{array}{l}\text { (Z)-5-Methylundec-4-en-6-yn- } \\
\text { 3-ol }\end{array}$ & $\mathrm{C}_{12} \mathrm{H}_{20} \mathrm{O}$ ? & $2326.55,3.770$ & 180.1509 & 15695 & $1.75 \mathrm{E}-4$ \\
\hline $\begin{array}{l}1,1^{\prime}-(1,4-B u t a n e d i y l) b i s- \\
\text { cyclohexane }\end{array}$ & $\mathrm{C}_{16} \mathrm{H}_{30}$ & $2326.55,4.036$ & 222.2342 & 50771 & \\
\hline (1,1-Dimethylbutyl)benzene & $\mathrm{C}_{12} \mathrm{H}_{18}$ & $2344.56,2.922$ & 162.1403 & 49213 & \\
\hline 1,4-Dipropylbenzene & $\mathrm{C}_{12} \mathrm{H}_{18}$ & $2356.56,2.898$ & 162.1403 & 32528 & \\
\hline $\begin{array}{l}\text { 1-Methyl-3-(2-methylpropyl)- } \\
\text { cyclopentane }\end{array}$ & $\mathrm{C}_{10} \mathrm{H}_{20}$ & $2362.56,3.991$ & 140.156 & 78948 & \\
\hline 1-Tridecene & $\mathrm{C}_{13} \mathrm{H}_{26}$ & $2380.57,4.154$ & 182.2029 & 102222 & \\
\hline 5-Methyltridecane & $\mathrm{C}_{14} \mathrm{H}_{30}$ & $2380.57,4.593$ & 198.2342 & 565349 & \\
\hline $\begin{array}{l}\text { [[1-Methyl-3-(2,6,6-trimethyl- } \\
\text { 1-cyclohexen-1-yl)-2- } \\
\text { propenyl]sulfonyl]benzene }\end{array}$ & $\mathrm{C}_{19} \mathrm{H}_{26} \mathrm{O}_{2} \mathrm{~S}$ & $2398.57,3.627$ & 318.1648 & 33931 & \\
\hline 2,6,11-Trimethyldodecane & $\mathrm{C}_{15} \mathrm{H}_{32}$ & $2404.58,4.834$ & 212.2499 & 440876 & \\
\hline $\begin{array}{l}\text { Dicyclohexyl- } \\
\text { acetalacetaldehyde }\end{array}$ & $\mathrm{C}_{14} \mathrm{H}_{26} \mathrm{O}_{2} ?$ & $2410.58,3.914$ & 226.1927 & 26972 & $2.56 \mathrm{E}-4$ \\
\hline $\begin{array}{l}\text { 1,7-Dimethyl-4-(1- } \\
\text { methylethyl)cyclodecane }\end{array}$ & $\mathrm{C}_{15} \mathrm{H}_{30}$ & $2410.58,4.223$ & 210.2342 & 298452 & \\
\hline 2-Methyltridecane & $\mathrm{C}_{14} \mathrm{H}_{30}$ & $2416.58,4.613$ & 198.2342 & 1189595 & \\
\hline
\end{tabular}




\begin{tabular}{|c|c|c|c|c|c|}
\hline 3,4-Dipropylcyclohex-3-enone & $\mathrm{C}_{12} \mathrm{H}_{20} \mathrm{O} ?$ & $2428.58,3.640$ & 180.1509 & 34177 & $3.24 \mathrm{E}-4$ \\
\hline Germacrane-B & $\mathrm{C}_{15} \mathrm{H}_{30}$ & $2434.58,4.199$ & 210.2342 & 92896 & \\
\hline $\begin{array}{l}\text { trans-1,2-Dibutylno } \\
\text { spacecyclopropane }\end{array}$ & $\mathrm{C}_{11} \mathrm{H}_{22}$ & $2440.59,4.132$ & 154.1716 & 47355 & \\
\hline $\begin{array}{l}\text { N-Butyl-N,N-di(2- } \\
\text { pyridiylmethyl)amine }\end{array}$ & $\mathrm{C}_{16} \mathrm{H}_{21} \mathrm{~N}_{3}$ & $2452.59,3.363$ & 255.173 & 9539 & \\
\hline 1-Methylcyclohexyl acetate & $\mathrm{C}_{9} \mathrm{H}_{16} \mathrm{O}_{2}$ & $2452.59,3.908$ & 156.1145 & 6649 & \\
\hline 3-Methyl-tridecane & $\mathrm{C}_{14} \mathrm{H}_{30}$ & $2458.59,4.531$ & 198.2342 & 1001169 & \\
\hline $\begin{array}{l}\text { 3- } \\
\text { Methyleneaminotricyclo[3.3.1. } \\
1(3,7)] \text { decane }\end{array}$ & $\mathrm{C}_{11} \mathrm{H}_{17} \mathrm{~N}$ & $2464.59,3.182$ & 163.1356 & 30410 & \\
\hline $\begin{array}{l}\text { trans-1-Ethyl-2-methyl } \\
\text { cyclohexane }\end{array}$ & $\mathrm{C}_{9} \mathrm{H}_{18}$ & $2476.6,4.217$ & 126.1403 & 78793 & \\
\hline 2-Methyl-1-cyclooctanol & $\mathrm{C}_{9} \mathrm{H}_{18} \mathrm{O}$ & $2536.61,4.128$ & 142.1352 & 67015 & \\
\hline $\begin{array}{l}\left(2 \mathrm{~S}, 2^{\prime} \mathrm{S}\right) \text { and }\left(2 \mathrm{~S}, 2^{\prime} \mathrm{R}\right)-\mathrm{N}-\left[2^{\prime}-\right. \\
\text { Hydroxy-2'-(p- } \\
\text { methylphenyl)ethyl]-2- } \\
\text { (methoxymethyl)pyrrolidine }\end{array}$ & $\mathrm{C}_{15} \mathrm{H}_{23} \mathrm{NO}_{2}$ & $2584.63,2.271$ & 249.1723 & 9419 & \\
\hline $\begin{array}{l}\text { trans-1-Butyl-2- } \\
\text { hexylcyclopropane }\end{array}$ & $\mathrm{C}_{13} \mathrm{H}_{26}$ & $2590.63,4.041$ & 182.2029 & 51004 & \\
\hline 1-Hexyl-3-methylcyclopentane & $\mathrm{C}_{12} \mathrm{H}_{24}$ & $2632.64,4.132$ & 168.1873 & 109985 & \\
\hline 1,1-Dimethyxyprop-2-yne & $\mathrm{C}_{5} \mathrm{H}_{8} \mathrm{O}_{2}$ ? & $2638.64,4.689$ & 100.0519 & 9419 & $1.80 \mathrm{E}-4$ \\
\hline $\begin{array}{l}\text { trans-1-Methyl-2-nonyl- } \\
\text { cyclohexane }\end{array}$ & $\mathrm{C}_{16} \mathrm{H}_{32}$ & $2680.65,4.063$ & 224.2499 & 283114 & \\
\hline 1-Azabicyclo[3.1.0]hexane & $\mathrm{C}_{5} \mathrm{H}_{9} \mathrm{~N}$ & $2692.66,4.024$ & 83.073 & 102160 & \\
\hline $\begin{array}{l}\text { 2,4,9,11-Tetramethyldodeca- } \\
\text { 5,7-diyne-4,9-diol }\end{array}$ & $\mathrm{C}_{16} \mathrm{H}_{26} \mathrm{O}_{2}$ & $2692.66,4.805$ & 250.1927 & 57363 & \\
\hline p-Methylpivalophenone & $\mathrm{C}_{12} \mathrm{H}_{16} \mathrm{O}$ & $2716.66,2.909$ & 176.1196 & 9820 & $1.51 \mathrm{E}-4$ \\
\hline 4-(Allyloxy)-2-butyne & $\mathrm{C}_{7} \mathrm{H}_{10} \mathrm{O}$ & $2722.67,3.933$ & 110.0726 & 13326 & \\
\hline $\begin{array}{l}\text { trans-1-(Cyclohexylmethyl)-3- } \\
\text { methylcyclohexane }\end{array}$ & $\mathrm{C}_{14} \mathrm{H}_{26}$ & $2734.67,3.988$ & 194.2029 & 149668 & \\
\hline $\begin{array}{l}\text { 5-Methyl-2,2,4-triethyl-2H- } \\
\text { imidazole }\end{array}$ & $\mathrm{C}_{10} \mathrm{H}_{18} \mathrm{~N}_{2}$ & $2734.67,4.232$ & 166.1465 & 10057 & \\
\hline 2,3-Dimethyl-2-butenoic acid & $\mathrm{C}_{6} \mathrm{H}_{10} \mathrm{O}_{2}$ & $2740.67,4.728$ & 114.0675 & 14597 & \\
\hline $\begin{array}{l}\text { 1,2,3,4-Tetrahydro-5- } \\
\text { methylnaphthalene }\end{array}$ & $\mathrm{C}_{11} \mathrm{H}_{14}$ & $2746.67,2.523$ & 146.109 & 51792 & \\
\hline 5-Propyldecane & $\mathrm{C}_{13} \mathrm{H}_{28}$ & $2746.67,4.544$ & 184.2186 & 1150816 & \\
\hline $\begin{array}{l}\text { 2,6,10,14-Tetramethyl- } \\
\text { heptadecane }\end{array}$ & $\mathrm{C}_{21} \mathrm{H}_{44}$ & $2752.68,4.762$ & 296.3438 & 3149250 & \\
\hline $\begin{array}{l}5,6,6- \\
\text { Trimethylbicyclo[3.2.0]heptan- } \\
\text { 2-one }\end{array}$ & $\mathrm{C}_{10} \mathrm{H}_{16} \mathrm{O} ?$ & $2758.68,4.254$ & 152.1196 & 27364 & $2.60 \mathrm{E}-4$ \\
\hline $\begin{array}{l}\text { 1,2,3,4-Tetrahydro-1,8- } \\
\text { dimethylnaphthalene }\end{array}$ & $\mathrm{C}_{12} \mathrm{H}_{16}$ & $2764.68,2.683$ & 160.1247 & 40724 & \\
\hline 10-Methylnonadecane & $\mathrm{C}_{20} \mathrm{H}_{42}$ & $2764.68,4.504$ & 282.3281 & 574911 & \\
\hline 2-Oxo-3-pentenenitrile & $\mathrm{C}_{5} \mathrm{H}_{5} \mathrm{NO}$ & $2782.68,3.659$ & 95.0366 & 9973 & \\
\hline
\end{tabular}




\begin{tabular}{|c|c|c|c|c|c|}
\hline 4-Methyltetradecane & $\mathrm{C}_{15} \mathrm{H}_{32}$ & $2782.68,4.535$ & 212.2499 & 513427 & \\
\hline 5-Methyl-3-hexyn-2-ol & $\mathrm{C}_{7} \mathrm{H}_{12} \mathrm{O}$ & $2824.7,4.194$ & 112.0883 & 28734 & \\
\hline 2,6,10-trimethyltridecane & $\mathrm{C}_{16} \mathrm{H}_{34}$ & $2824.7,4.435$ & 226.2655 & 76122 & \\
\hline 3-Methyltetradecane & $\mathrm{C}_{15} \mathrm{H}_{32}$ & $2836.7,4.469$ & 212.2499 & 879617 & \\
\hline $\begin{array}{l}\text { 1,2,3,4-Tetrahydro-1,4- } \\
\text { dimethylnaphthalene }\end{array}$ & $\mathrm{C}_{12} \mathrm{H}_{16}$ & $2902.72,2.636$ & 160.1247 & 36499 & \\
\hline Octylcyclohexane & $\mathrm{C}_{14} \mathrm{H}_{28}$ & $2908.72,3.883$ & 196.2186 & 713244 & \\
\hline Tetradecylcyclooctane & $\mathrm{C}_{22} \mathrm{H}_{44}$ & $2926.73,4.071$ & 308.3438 & 123702 & \\
\hline 2-Pent-1-enyltetrahydrofuran & $\mathrm{C}_{9} \mathrm{H}_{16} \mathrm{O}$ & $2938.73,4.082$ & 140.1196 & 41779 & \\
\hline Nonadecane & $\mathrm{C}_{19} \mathrm{H}_{40}$ & $2974.74,4.572$ & 268.3125 & 512729 & \\
\hline Heptane & $\mathrm{C}_{7} \mathrm{H}_{16}$ & $3004.75,4.743$ & 100.1247 & 24876 & \\
\hline $\begin{array}{l}\text { 4-(3-Hydroxy-3-methyl- } \\
\text { butenyl)imidazole }\end{array}$ & $\mathrm{C}_{8} \mathrm{H}_{12} \mathrm{~N}_{2} \mathrm{O}$ & $3016.75,2.831$ & 152.0944 & 5118 & \\
\hline 5-Phenylpent-1-en-3-yne & $\mathrm{C}_{11} \mathrm{H}_{10}$ & $3022.75,2.314$ & 142.0777 & 83346 & \\
\hline 2-Methyl-3-oxazoline & $\mathrm{C}_{4} \mathrm{H}_{7} \mathrm{NO}$ & $3028.75,4.536$ & 85.0522 & 12051 & \\
\hline Eicosane & $\mathrm{C}_{20} \mathrm{H}_{42}$ & $3034.76,4.517$ & 282.3281 & 100199 & \\
\hline $\begin{array}{l}\text { Indan-9-ethoxy-7a,3a - } \\
\text { (nitrilometheno) }\end{array}$ & $\mathrm{C}_{12} \mathrm{H}_{15} \mathrm{NO} ?$ & $3076.77,2.831$ & 189.1148 & 10677 & $3.05 \mathrm{E}-4$ \\
\hline 6-Methylpentadecane & $\mathrm{C}_{16} \mathrm{H}_{34}$ & $3112.78,4.454$ & 226.2655 & 1519679 & \\
\hline $\begin{array}{l}\text { 1-n-Butyl-1,2,3,4- } \\
\text { tetrahydronaphthalene }\end{array}$ & $\mathrm{C}_{14} \mathrm{H}_{20}$ & $3118.78,2.527$ & 188.156 & 9410 & \\
\hline 1-Methyl-2-pyrazoline & $\mathrm{C}_{4} \mathrm{H}_{8} \mathrm{~N}_{2}$ & $3118.78,4.263$ & 84.0682 & 27072 & \\
\hline 1-Methylnaphthalene & $\mathrm{C}_{11} \mathrm{H}_{10}$ & $3124.78,2.278$ & 142.0777 & 39716 & \\
\hline 3-Ethylcosane & $\mathrm{C}_{22} \mathrm{H}_{46}$ & $3124.78,4.640$ & 310.3594 & 121462 & \\
\hline 4-Methylpentadecane & $\mathrm{C}_{16} \mathrm{H}_{34}$ & $3148.79,4.449$ & 226.2655 & 589448 & \\
\hline $\begin{array}{l}\text { Hexahydro-1H-pyrrolizin-1- } \\
\text { one }\end{array}$ & $\mathrm{C}_{7} \mathrm{H}_{11} \mathrm{NO} ?$ & $3154.79,3.865$ & 125.0835 & 19094 & $5.73 \mathrm{E}-4$ \\
\hline 3,3,4,4-Tetraethylhexane & $\mathrm{C}_{14} \mathrm{H}_{30}$ & $3190.8,4.576$ & 198.2342 & 89557 & \\
\hline 3-Methylpentadecane & $\mathrm{C}_{16} \mathrm{H}_{34}$ & $3202.8,4.407$ & 226.2655 & 672137 & \\
\hline 1-Octadecanol & $\mathrm{C}_{18} \mathrm{H}_{38} \mathrm{O}$ ? & $3208.81,4.072$ & 270.2917 & 26911 & $2.55 \mathrm{E}-4$ \\
\hline 1-Buten-3,4-dicarboxylic acid & $\mathrm{C}_{6} \mathrm{H}_{8} \mathrm{O}_{4}$ & $3208.81,4.587$ & 144.0417 & 89180 & \\
\hline (E)-2-Phenyl-3-octene & $\mathrm{C}_{14} \mathrm{H}_{20}$ & $3256.82,2.784$ & 188.156 & 12334 & \\
\hline $\begin{array}{l}\text { 1,2,3,4-Tetrahydro-5,7- } \\
\text { dimethylnaphthalene }\end{array}$ & $\mathrm{C}_{12} \mathrm{H}_{16}$ & $3274.82,2.503$ & 160.1247 & 46516 & \\
\hline $\begin{array}{l}\text { 1-Cyclohexyl-3-methyl- } \\
\text { benzene }\end{array}$ & $\mathrm{C}_{13} \mathrm{H}_{18}$ & $3286.83,2.657$ & 174.1403 & 37394 & \\
\hline
\end{tabular}




\begin{tabular}{|c|c|c|c|c|c|}
\hline n-Nonylcyclohexane & $\mathrm{C}_{15} \mathrm{H}_{30}$ & $3286.83,3.842$ & 210.2342 & 558527 & \\
\hline 6-Hydroxy-3-hexanone & $\mathrm{C}_{6} \mathrm{H}_{12} \mathrm{O}_{2}$ & $3310.84,4.541$ & 116.0832 & 252832 & \\
\hline 9-Hydroxyinalol & $\mathrm{C}_{10} \mathrm{H}_{18} \mathrm{O}_{2}$ ? & $3376.85,4.659$ & 170.1301 & 24150 & $2.29 \mathrm{E}-4$ \\
\hline 1-Ethylnaphthalene & $\mathrm{C}_{12} \mathrm{H}_{12}$ & $3382.86,2.369$ & 156.0934 & 12256 & \\
\hline cis-Bicyclo[3.3.0]octan-1-ol & $\mathrm{C}_{8} \mathrm{H}_{14} \mathrm{O}$ & $3388.86,3.563$ & 126.1039 & 19773 & \\
\hline $\begin{array}{l}\text { (Z)-2-(4,4-Dimethyl-2-oxo-2- } \\
\text { C13-dihydrofuran-3(2H)- } \\
\text { ylidene)acetic acid }\end{array}$ & $\mathrm{C}_{8} \mathrm{H}_{10} \mathrm{O}_{4}$ & $3394.86,4.619$ & 170.0574 & 6673 & \\
\hline 1,7-Dimethylnaphthalene & $\mathrm{C}_{12} \mathrm{H}_{12}$ & $3418.87,2.386$ & 156.0934 & 154622 & \\
\hline 2-Methoxymethylfuran & $\mathrm{C}_{6} \mathrm{H}_{8} \mathrm{O}_{2}$ & $3460.88,4.089$ & 112.0519 & 22629 & \\
\hline 2,6,8-Trimethyldecane & $\mathrm{C}_{13} \mathrm{H}_{28}$ & $3460.88,4.397$ & 184.2186 & 1465120 & \\
\hline $\begin{array}{l}\text { 1-(1-Cyclopentenyl)-1- } \\
\text { hydroxy-5,5-dimethyl-6- } \\
\text { hepten-3-one }\end{array}$ & $\mathrm{C}_{14} \mathrm{H}_{22} \mathrm{O}_{2}$ ? & $3478.88,4.144$ & 222.1614 & 27327 & $2.59 \mathrm{E}-4$ \\
\hline 2,7-Dimethylundecane & $\mathrm{C}_{13} \mathrm{H}_{28}$ & $3478.88,4.392$ & 184.2186 & 510633 & \\
\hline Hexahydro-1H-pyrrolizine & $\mathrm{C}_{7} \mathrm{H}_{13} \mathrm{~N}$ & $3490.89,4.022$ & 111.1043 & 7551 & \\
\hline $\begin{array}{l}\text { 4-Methyl-2-propyl-2,5- } \\
\text { dihydrofuran }\end{array}$ & $\mathrm{C}_{8} \mathrm{H}_{14} \mathrm{O}$ & $3496.89,3.902$ & 126.1039 & 37125 & \\
\hline 4-Methylhexadecane & $\mathrm{C}_{17} \mathrm{H}_{36}$ & $3496.89,4.392$ & 240.2812 & 394562 & \\
\hline 1,5-Dimethylnaphthalene & $\mathrm{C}_{12} \mathrm{H}_{12}$ & $3502.89,2.350$ & 156.0934 & 61922 & \\
\hline 2-Methyloctadecane & $\mathrm{C}_{19} \mathrm{H}_{40}$ & $3514.89,4.392$ & 268.3125 & 691076 & \\
\hline $\begin{array}{l}\text { 3-Hydroxy-7,7-dimethyl-1,8- } \\
\text { nonadien-5-one }\end{array}$ & $\mathrm{C}_{11} \mathrm{H}_{18} \mathrm{O}_{2}$ ? & $3526.9,4.117$ & 182.1301 & 84877 & $8.06 \mathrm{E}-4$ \\
\hline 3-Methylhexadecane & $\mathrm{C}_{17} \mathrm{H}_{36}$ & $3550.9,4.335$ & 240.2812 & 509525 & \\
\hline 1,3-Dimethylnaphthalene & $\mathrm{C}_{12} \mathrm{H}_{12}$ & $3598.92,2.326$ & 156.0934 & 20139 & \\
\hline $\begin{array}{l}\text { 1-(2- } \\
\text { Methoxyethenyl)cyclopropane }\end{array}$ & $\mathrm{C}_{6} \mathrm{H}_{10} \mathrm{O}$ & $3598.92,4.113$ & 98.0726 & 15579 & \\
\hline Cyclopentanodec-5-ene & $\mathrm{C}_{15} \mathrm{H}_{28}$ & $3610.92,4.028$ & 208.2186 & 108355 & \\
\hline 1-Heptacosanol & $\mathrm{C}_{27} \mathrm{H}_{56} \mathrm{O}$ ? & $3616.92,4.007$ & 396.4326 & 40443 & $3.84 \mathrm{E}-4$ \\
\hline 2,4,4-Trimethylhexane & $\mathrm{C}_{9} \mathrm{H}_{20}$ & $3616.92,4.675$ & 128.156 & 424257 & \\
\hline 1,8-Dimethylnaphthalene & $\mathrm{C}_{12} \mathrm{H}_{12}$ & $3622.92,2.289$ & 156.0934 & 10031 & \\
\hline Undecylcyclohexane & $\mathrm{C}_{17} \mathrm{H}_{34}$ & $3652.93,3.816$ & 238.2655 & 406360 & \\
\hline n-Cetyl thiocyanate & $\mathrm{C}_{17} \mathrm{H}_{33} \mathrm{NS}$ & $3712.95,4.367$ & 283.2328 & 133265 & \\
\hline $\begin{array}{l}\text { 1-Oxa-spiro[5.2] octane-2- } \\
\text { carbaldehyde }\end{array}$ & $\mathrm{C}_{8} \mathrm{H}_{12} \mathrm{O}_{2}$ & $3718.95,4.143$ & 140.0832 & 51740 & \\
\hline 1-Heptadecanol & $\mathrm{C}_{17} \mathrm{H}_{36} \mathrm{O}$ ? & $3736.96,3.994$ & 256.2761 & 70284 & $6.67 \mathrm{E}-4$ \\
\hline
\end{tabular}




\begin{tabular}{|c|c|c|c|c|c|}
\hline 2-Methylene-3-butenylamine & $\mathrm{C}_{5} \mathrm{H}_{9} \mathrm{~N}$ & $3754.96,3.977$ & 83.073 & 97531 & \\
\hline 3-Methyltricosane & $\mathrm{C}_{24} \mathrm{H}_{50}$ & $3754.96,4.339$ & 338.3907 & 56426 & \\
\hline 2-Methyl-1,1'-biphenyl & $\mathrm{C}_{13} \mathrm{H}_{12}$ & $3772.97,2.268$ & 168.0934 & 40384 & \\
\hline $\begin{array}{l}\left(1 \mathrm{R}^{*}, 5 \mathrm{~S}^{*}, 7 \mathrm{R}^{*}\right)-7- \\
\text { Methylbicyclo[3.2.1] } \mathrm{octan}-1-\mathrm{ol}\end{array}$ & $\mathrm{C}_{9} \mathrm{H}_{16} \mathrm{O}$ & $3778.97,4.057$ & 140.1196 & 44399 & \\
\hline $\begin{array}{l}\text { 2-(1-Cyclohexyl-1- } \\
\text { methylethyl)propanedicarbonit } \\
\text { rile }\end{array}$ & $\mathrm{C}_{12} \mathrm{H}_{18} \mathrm{~N}_{2}$ & $3790.97,4.121$ & 190.1465 & 25017 & \\
\hline $\begin{array}{l}\text { 2-Isobutyl-3- } \\
\text { methylcyclopentan-1-one }\end{array}$ & $\mathrm{C}_{10} \mathrm{H}_{18} \mathrm{O}$ & $3796.97,4.034$ & 154.1352 & 68369 & \\
\hline $\begin{array}{l}\text { Phenyl ethyl } \\
\text { benzeneethanimidate }\end{array}$ & $\mathrm{C}_{16} \mathrm{H}_{17} \mathrm{NO} ?$ & $3814.98,2.273$ & 239.1305 & 15880 & $1.50 \mathrm{E}-4$ \\
\hline 4-Methylheptadecane & $\mathrm{C}_{18} \mathrm{H}_{38}$ & $3826.98,4.320$ & 254.2968 & 222456 & \\
\hline 2-Methyleicosane & $\mathrm{C}_{21} \mathrm{H}_{44}$ & $3838.99,4.335$ & 296.3438 & 743644 & \\
\hline 2,3,6-Trimethylnaphthalene & $\mathrm{C}_{13} \mathrm{H}_{14}$ & $3869,2.386$ & 170.109 & 76351 & \\
\hline 1,6,7-trimethyl-naphthalene & $\mathrm{C}_{13} \mathrm{H}_{14}$ & $3881,2.397$ & 170.109 & 83936 & \\
\hline 3,8-Dimethylundecane & $\mathrm{C}_{13} \mathrm{H}_{28}$ & $3881,4.284$ & 184.2186 & 445486 & \\
\hline 2-p-Tolyl-1H-pyrrole & $\mathrm{C}_{11} \mathrm{H}_{11} \mathrm{~N}$ & $3911.01,2.649$ & 157.0886 & 9668 & \\
\hline 2,2,3,3-Tetramethylbutane & $\mathrm{C}_{8} \mathrm{H}_{18}$ & $3911.01,4.644$ & 114.1403 & 85702 & \\
\hline 2,2-Dimethylbutane & $\mathrm{C}_{6} \mathrm{H}_{14}$ & $3953.02,4.449$ & 86.109 & 153113 & \\
\hline 6-Allyltetrahydropyran-2-one & $\mathrm{C}_{8} \mathrm{H}_{12} \mathrm{O}_{2}$ & $3959.02,4.436$ & 140.0832 & 82478 & \\
\hline 1,4,6-Trimethylnaphthalene & $\mathrm{C}_{13} \mathrm{H}_{14}$ & $3965.02,2.381$ & 170.109 & 86101 & \\
\hline 3,3'-Dimethyl-1,1'-biphenyl & $\mathrm{C}_{14} \mathrm{H}_{14}$ & $4121.07,2.305$ & 182.109 & 82943 & \\
\hline 3,4'-Dimethyl-1,1'-biphenyl & $\mathrm{C}_{14} \mathrm{H}_{14}$ & $4163.08,2.309$ & 182.109 & 43064 & \\
\hline (E)-Deca-1,6-dien-3-one & $\mathrm{C}_{10} \mathrm{H}_{16} \mathrm{O}$ & $4169.08,4.579$ & 152.1196 & 20175 & \\
\hline $\begin{array}{l}2,6,10,15- \\
\text { Tetramethylheptadecane }\end{array}$ & $\mathrm{C}_{21} \mathrm{H}_{44}$ & $4181.09,4.608$ & 296.3438 & 917232 & \\
\hline Heptacosane & $\mathrm{C}_{27} \mathrm{H}_{56}$ & $4193.09,4.243$ & 380.4377 & 344773 & \\
\hline $\begin{array}{l}\text { trans--1-Butyl-2- } \\
\text { undecylcyclopropane }\end{array}$ & $\mathrm{C}_{18} \mathrm{H}_{36}$ & $4367.14,3.933$ & 252.2812 & 52121 & \\
\hline $\begin{array}{l}\text { 1-(1-Methylcyclohexyl)- } \\
\text { ethanone }\end{array}$ & $\mathrm{C}_{9} \mathrm{H}_{16} \mathrm{O}$ & $4415.15,3.898$ & 140.1196 & 153780 & \\
\hline 2,4,6-Trimethyl-1,1'-biphenyl & $\mathrm{C}_{15} \mathrm{H}_{16}$ & $4433.16,2.340$ & 196.1247 & 53978 & \\
\hline 3,7-Dimethyldecane & $\mathrm{C}_{12} \mathrm{H}_{26}$ & $4445.16,4.243$ & 170.2029 & 137176 & \\
\hline (E)-3-Octen-2,5-dione & $\mathrm{C}_{8} \mathrm{H}_{12} \mathrm{O}_{2}$ ? & $4475.17,3.850$ & 140.0832 & 98937 & $2.47 \mathrm{E}-3$ \\
\hline 3,3-Dimethylheptane & $\mathrm{C}_{9} \mathrm{H}_{20}$ & $4565.2,4.416$ & 128.156 & 72944 & \\
\hline 1-Methoxybicyclo(3.2.1)octane & $\mathrm{C}_{9} \mathrm{H}_{16} \mathrm{O}$ & $4655.22,3.927$ & 140.1196 & 66873 & \\
\hline
\end{tabular}




\begin{tabular}{|c|c|c|c|c|c|}
\hline 2-Methylpyrrolizidine & $\mathrm{C}_{8} \mathrm{H}_{15} \mathrm{~N}$ & $4673.23,3.948$ & 125.1199 & 25486 & \\
\hline Pentatriacontane & $\mathrm{C}_{35} \mathrm{H}_{72}$ & $4673.23,4.263$ & 492.5629 & 466027 & \\
\hline 2,2,3,3-Tetramethylhexane & $\mathrm{C}_{10} \mathrm{H}_{22}$ & $4709.24,4.243$ & 142.1716 & 70431 & \\
\hline $\begin{array}{l}\text { 2-Methyl-4-methylidenehex-5- } \\
\text { en-3-ol }\end{array}$ & $\mathrm{C}_{8} \mathrm{H}_{14} \mathrm{O}$ & $4889.29,3.784$ & 126.1039 & 13097 & \\
\hline 4-Methylstilbene & $\mathrm{C}_{15} \mathrm{H}_{14}$ & $4967.31,2.292$ & 194.109 & 10224 & \\
\hline $\begin{array}{l}\text { anti-9-Methyl-1,6- } \\
\text { methanofluorene }\end{array}$ & $\mathrm{C}_{15} \mathrm{H}_{14}$ & $4985.32,2.294$ & 194.109 & 10067 & \\
\hline Heneicosane & $\mathrm{C}_{21} \mathrm{H}_{44}$ & $4985.32,4.223$ & 296.3438 & 50325 & \\
\hline Benzo[A]azulene & $\mathrm{C}_{14} \mathrm{H}_{10}$ & $5027.33,2.124$ & 178.0777 & 26705 & \\
\hline $\begin{array}{l}\text { 1-Hydroxy-2-methyl- } \\
\text { bicyclo[3.3.1]nonan-3-one }\end{array}$ & $\mathrm{C}_{10} \mathrm{H}_{16} \mathrm{O}_{2}$ ? & $5273.4,3.851$ & 168.1145 & 64185 & $6.09 \mathrm{E}-4$ \\
\hline 2,10-Dimethylundecane & $\mathrm{C}_{13} \mathrm{H}_{28}$ & $5309.41,4.193$ & 184.2186 & 137521 & \\
\hline 4-Methylphenanthrene & $\mathrm{C}_{15} \mathrm{H}_{12}$ & $5327.41,2.193$ & 192.0934 & 18641 & \\
\hline (E)-5-Heptenenitrile & $\mathrm{C}_{7} \mathrm{H}_{11} \mathrm{~N}$ & $5339.42,3.820$ & 109.0886 & 17066 & \\
\hline $\begin{array}{l}8,9- \\
\text { Dihydrocyclopent[a]acenaphth } \\
\text { ylene, }\end{array}$ & $\mathrm{C}_{15} \mathrm{H}_{12}$ & $5357.42,2.181$ & 192.0934 & 22597 & \\
\hline $\begin{array}{l}\text { 1H-1a,9b-dihydro- } \\
\text { cyclopropa[1]phenanthrene }\end{array}$ & $\mathrm{C}_{15} \mathrm{H}_{12}$ & $5441.45,2.175$ & 192.0934 & 8827 & \\
\hline Docosane & $\mathrm{C}_{22} \mathrm{H}_{46}$ & $5459.45,4.221$ & 310.3594 & 142439 & \\
\hline 2,2,4,6,6-Pentamethyl heptane & $\mathrm{C}_{12} \mathrm{H}_{26}$ & $5561.48,4.196$ & 170.2029 & 77571 & \\
\hline 2,2-Dimethylpropyl nitrite & $\mathrm{C}_{5} \mathrm{H}_{11} \mathrm{NO}_{2}$ & $6791.83,4.402$ & 117.0784 & 21843 & \\
\hline Tetracosane & $\mathrm{C}_{24} \mathrm{H}_{50}$ & $6941.88,4.392$ & 338.3907 & 111796 & \\
\hline 4-Hydroxyhex-2-en-1-al & $\mathrm{C}_{6} \mathrm{H}_{10} \mathrm{O}_{2}$ & $7139.93,4.512$ & 114.0675 & 42626 & \\
\hline Benzene & $\mathrm{C}_{6} \mathrm{H}_{6}$ & $7163.94,1.098$ & 78.0464 & 11743 & \\
\hline $\begin{array}{l}\text { 2,2-Dimethyl-3-butyl-4-(2'- } \\
\text { propenyl)-2,5-dihydrofuran }\end{array}$ & $\mathrm{C}_{13} \mathrm{H}_{22} \mathrm{O}$ ? & $7788.12,1.202$ & 194.1665 & 24815 & $2.35 \mathrm{E}-4$ \\
\hline $\begin{array}{l}\text { 2-(1,1-Dimethylprop-2-en-1- } \\
\text { yl)-1,3-dioxolane }\end{array}$ & $\mathrm{C}_{8} \mathrm{H}_{14} \mathrm{O}_{2}$ & $7998.18,1.162$ & 142.0988 & 19952 & \\
\hline $\begin{array}{l}\text { 2-(3,3-Dimethyl-1-butenyl)- } \\
\text { (E)-1-cyclohexene-1- } \\
\text { carboxaldehyde }\end{array}$ & $\mathrm{C}_{13} \mathrm{H}_{20} \mathrm{O}$ & $8076.2,1.375$ & 192.1509 & 8089 & \\
\hline
\end{tabular}


Table S17. Data obtained for the distillate fraction of condensate-like oil 4 by using GCxGC/(+EI) TOF MS. Data provided are based on ChromaTOF software prediction of the molecular ion $\mathrm{m} / \mathrm{z}$ value and the identification of the compound by using the EI mass spectral library match using a match factor threshold of 800. Formulas with a question mark "?" might not have been accurately identified. The ratio of the peak area of each of these questionable compounds relative to all peak areas of the detected compounds is provided in the table below

\begin{tabular}{|c|c|c|c|c|c|}
\hline Name & Formula & $\begin{array}{l}\text { Retention Times (s) on } \\
\text { the Primary, Secondary } \\
\text { Column }\end{array}$ & $\begin{array}{l}\text { Molecular } \\
\text { Ion } \mathbf{~} / \mathbf{z}\end{array}$ & $\begin{array}{c}\text { Chromatographic } \\
\text { Peak Area }\end{array}$ & $\begin{array}{c}\text { Ratio } \\
\text { (Peak } \\
\text { area/total } \\
\text { sum of peak } \\
\text { areas) } \\
\end{array}$ \\
\hline 3-Methylheptane & $\mathrm{C}_{8} \mathrm{H}_{18}$ & $406.002,2.407$ & 114.1403 & 345175 & \\
\hline Isooctanol & $\mathrm{C}_{8} \mathrm{H}_{18} \mathrm{O}$ & $430.009,2.300$ & 130.1352 & 66947 & \\
\hline 1-Buten-1-one & $\mathrm{C}_{4} \mathrm{H}_{6} \mathrm{O}$ & $436.01,2.373$ & 70.0413 & 26391 & \\
\hline Octane & $\mathrm{C}_{8} \mathrm{H}_{18}$ & $442.012,2.597$ & 114.1403 & 805143 & \\
\hline cis-1,3-Dimethylcyclohexane & $\mathrm{C}_{8} \mathrm{H}_{16}$ & $454.016,2.386$ & 112.1247 & 2614525 & \\
\hline 2-Methyl-1-pentanol & $\mathrm{C}_{6} \mathrm{H}_{14} \mathrm{O}$ & $454.016,2.597$ & 102.1039 & 18640 & \\
\hline 2,4-Dimethylhexane & $\mathrm{C}_{8} \mathrm{H}_{18}$ & $460.017,2.793$ & 114.1403 & 361560 & \\
\hline Di(2-ethylbutyl) ether & $\mathrm{C}_{12} \mathrm{H}_{26} \mathrm{O} ?$ & $472.021,2.826$ & 186.1978 & 66954 & $9.11 \mathrm{E}-4$ \\
\hline 1,1-Dimethylcyclohexane & $\mathrm{C}_{8} \mathrm{H}_{16}$ & $478.022,2.422$ & 112.1247 & 457605 & \\
\hline 2,6-Dimethylheptane & $\mathrm{C}_{9} \mathrm{H}_{20}$ & $478.022,2.875$ & 128.156 & 468932 & \\
\hline (S)-(+)-2-Acetoxyoctane & $\mathrm{C}_{10} \mathrm{H}_{20} \mathrm{O}_{2}$ ? & $484.024,2.489$ & 172.1458 & 14487 & $1.97 \mathrm{E}-4$ \\
\hline trans-1,2-Dimethylcyclohexane & $\mathrm{C}_{8} \mathrm{H}_{16}$ & $496.028,2.497$ & 112.1247 & 647324 & \\
\hline 3,5-Dimethylno hyphenheptane & $\mathrm{C}_{9} \mathrm{H}_{20}$ & $496.028,2.916$ & 128.156 & 1310843 & \\
\hline trans-1,3-Dimethylcyclohexane & $\mathrm{C}_{8} \mathrm{H}_{16}$ & $514.033,2.530$ & 112.1247 & 648656 & \\
\hline (+-)-4-Vinyldecanal & $\mathrm{C}_{12} \mathrm{H}_{22} \mathrm{O}$ ? & $514.033,2.767$ & 182.1665 & 8349 & $1.14 \mathrm{E}-4$ \\
\hline 1,3,5-Trimethylcyclohexane & $\mathrm{C}_{9} \mathrm{H}_{18}$ & $538.04,2.772$ & 126.1403 & 362435 & \\
\hline 5-Methyldecane & $\mathrm{C}_{11} \mathrm{H}_{24}$ & $550.043,3.107$ & 156.1873 & 174985 & \\
\hline 1,1,3-Trimethylcyclohexane & $\mathrm{C}_{9} \mathrm{H}_{18}$ & $562.047,2.803$ & 126.1403 & 846536 & \\
\hline 2,4-Dimethylheptane & $\mathrm{C}_{9} \mathrm{H}_{20}$ & $562.047,3.137$ & 128.156 & 1077895 & \\
\hline 3-Methyloctane & $\mathrm{C}_{9} \mathrm{H}_{20}$ & $580.052,3.189$ & 128.156 & 1956654 & \\
\hline Ethylcyclohexane & $\mathrm{C}_{8} \mathrm{H}_{16}$ & $586.053,2.685$ & 112.1247 & 751674 & \\
\hline 1,2,4-Trimethylcyclohexane & $\mathrm{C}_{9} \mathrm{H}_{18}$ & $592.055,2.906$ & 126.1403 & 636493 & \\
\hline
\end{tabular}




\begin{tabular}{|c|c|c|c|c|c|}
\hline Methylbenzene & $\mathrm{C}_{7} \mathrm{H}_{8}$ & $598.057,2.057$ & 92.0621 & 138021 & \\
\hline Heptane & $\mathrm{C}_{7} \mathrm{H}_{16}$ & $628.065,3.495$ & 100.1247 & 32149 & \\
\hline 3-Butyl-3,5-dimethylcyclohexanone & $\mathrm{C}_{12} \mathrm{H}_{22} \mathrm{O}$ ? & $658.074,3.185$ & 182.1665 & 121589 & $1.65 \mathrm{E}-4$ \\
\hline Nonane & $\mathrm{C}_{9} \mathrm{H}_{20}$ & $658.074,3.384$ & 128.156 & 1730365 & \\
\hline 1,2,3-Trimethylcyclohexane & $\mathrm{C}_{9} \mathrm{H}_{18}$ & $670.078,3.022$ & 126.1403 & 218158 & \\
\hline 1-Hydroxy-3-penten-2-one & $\mathrm{C}_{5} \mathrm{H}_{8} \mathrm{O}_{2}$ & $676.079,3.348$ & 100.0519 & 8841 & \\
\hline 2,4,6-Trimethylheptane & $\mathrm{C}_{10} \mathrm{H}_{22}$ & $682.081,3.636$ & 142.1716 & 669735 & \\
\hline 1-Ethyl-4-methylcyclohexane & $\mathrm{C}_{9} \mathrm{H}_{18}$ & $706.088,3.096$ & 126.1403 & 1193567 & \\
\hline 2,5-Dimethyloctane & $\mathrm{C}_{10} \mathrm{H}_{22}$ & $706.088,3.682$ & 142.1716 & 1035019 & \\
\hline 3,6-Dimethyloctane & $\mathrm{C}_{10} \mathrm{H}_{22}$ & $736.096,3.734$ & 142.1716 & 979910 & \\
\hline trans-1-Ethyl-4-methyl cyclohexane & $\mathrm{C}_{9} \mathrm{H}_{18}$ & $778.109,3.168$ & 126.1403 & 512119 & \\
\hline 4,5-Dimethyloctane & $\mathrm{C}_{10} \mathrm{H}_{22}$ & $790.112,3.784$ & 142.1716 & 53327 & \\
\hline 1-Ethyl-1-methylcyclohexane & $\mathrm{C}_{9} \mathrm{H}_{18}$ & $808.117,3.131$ & 126.1403 & 132873 & \\
\hline 4-Ethyloctane & $\mathrm{C}_{10} \mathrm{H}_{22}$ & $814.119,3.837$ & 142.1716 & 762796 & \\
\hline Dodecane & $\mathrm{C}_{12} \mathrm{H}_{26}$ & $832.124,3.898$ & 170.2029 & 1896380 & \\
\hline Diethylcyclohexane & $\mathrm{C}_{10} \mathrm{H}_{20}$ & $838.126,3.487$ & 140.156 & 285406 & \\
\hline 5H-Tetrazol-5-amine & $\mathrm{CH}_{3} \mathrm{~N}_{5}$ & $844.127,4.171$ & 85.0383 & 174881 & \\
\hline 2,3,3-Trimethyl-4-nonene & $\mathrm{C}_{12} \mathrm{H}_{24}$ & $850.129,3.485$ & 168.1873 & 165314 & \\
\hline Propylcyclohexane, & $\mathrm{C}_{9} \mathrm{H}_{18}$ & $856.131,3.266$ & 126.1403 & 419216 & \\
\hline 3-Methylnonane & $\mathrm{C}_{10} \mathrm{H}_{22}$ & $868.134,3.919$ & 142.1716 & 1061294 & \\
\hline cis-Octahydroindene & $\mathrm{C}_{9} \mathrm{H}_{16}$ & $874.136,3.099$ & 124.1247 & 85930 & \\
\hline $\begin{array}{l}\text { 1-Methyl-2-methylene-3-(1- } \\
\text { methylethyl)cyclopentane }\end{array}$ & $\mathrm{C}_{10} \mathrm{H}_{18}$ & $880.138,3.349$ & 138.1403 & 45149 & \\
\hline Ethylbenzene & $\mathrm{C}_{8} \mathrm{H}_{10}$ & $886.14,2.369$ & 106.0777 & 30331 & \\
\hline 8-Methyl-1,7-nonadien-4-one & $\mathrm{C}_{10} \mathrm{H}_{16} \mathrm{O}$ ? & $898.143,3.482$ & 152.1196 & 89535 & $1.21 \mathrm{E}-3$ \\
\hline o-Xylene & $\mathrm{C}_{8} \mathrm{H}_{10}$ & $904.145,2.412$ & 106.0777 & 418068 & \\
\hline 2-Eethyl-1H-imidazole & $\mathrm{C}_{5} \mathrm{H}_{8} \mathrm{~N}_{2}$ & $904.145,3.344$ & 96.0682 & 10520 & \\
\hline 4-Methylundecane & $\mathrm{C}_{12} \mathrm{H}_{26}$ & $916.148,4.240$ & 170.2029 & 27620 & \\
\hline trans-1-Ethyl-1,3-dimethyl cyclohexane & $\mathrm{C}_{10} \mathrm{H}_{20}$ & $922.15,3.559$ & 140.156 & 483840 & \\
\hline Decane & $\mathrm{C}_{10} \mathrm{H}_{22}$ & $976.165,4.053$ & 142.1716 & 2846145 & \\
\hline
\end{tabular}




\begin{tabular}{|c|c|c|c|c|c|}
\hline 1-Isopropyl-4-methylcyclohexane & $\mathrm{C}_{10} \mathrm{H}_{20}$ & $982.167,3.579$ & 140.156 & 229053 & \\
\hline $\begin{array}{l}\text { 2,4-Bis(dimethylethyl)-2-methyl-2,5- } \\
\text { dihydro-1H-imidazole }\end{array}$ & $\mathrm{C}_{12} \mathrm{H}_{24} \mathrm{~N}_{2}$ & $982.167,3.837$ & 196.1934 & 39775 & \\
\hline 1-Methyl-3-propylcyclohexane & $\mathrm{C}_{10} \mathrm{H}_{20}$ & $1006.17,3.651$ & 140.156 & 907920 & \\
\hline 13,13-Diethoxy-(Z)-1,11-tridecadiene & $\mathrm{C}_{17} \mathrm{H}_{32} \mathrm{O}_{2} ?$ & $1012.18,3.569$ & 268.2397 & 41253 & $5.61 \mathrm{E}-4$ \\
\hline $\begin{array}{l}\text { (E)-1-((1'S,2'S)-trans-2- } \\
\text { (Methyl)cyclopentyl)-2-butylethylene }\end{array}$ & $\mathrm{C}_{12} \mathrm{H}_{22}$ & $1018.18,3.441$ & 166.1716 & 26423 & \\
\hline 1,2-Dimethylbenzene & $\mathrm{C}_{8} \mathrm{H}_{10}$ & $1024.18,2.417$ & 106.0777 & 170341 & \\
\hline 2,6,7-Trimethyldecane & $\mathrm{C}_{13} \mathrm{H}_{28}$ & $1024.18,4.330$ & 184.2186 & 1143855 & \\
\hline 2,7,10-Trimethyldodecane & $\mathrm{C}_{15} \mathrm{H}_{32}$ & $1078.19,4.356$ & 212.2499 & 364035 & \\
\hline Methyl p-methylbenzyl sulfoxide & $\mathrm{C}_{9} \mathrm{H}_{12} \mathrm{OS}$ & $1102.2,2.570$ & 168.0603 & 11736 & \\
\hline Tetradecane & $\mathrm{C}_{14} \mathrm{H}_{30}$ & $1102.2,4.346$ & 198.2342 & 327260 & \\
\hline $\begin{array}{l}\text { 1-Hydroxymethyl-1,3-dimethyl- } \\
\text { cyclopentane }\end{array}$ & $\mathrm{C}_{8} \mathrm{H}_{16} \mathrm{O}$ & $1114.2,3.646$ & 128.1196 & 28619 & \\
\hline 1-Cyclopropyl-2-propen-1-one & $\mathrm{C}_{6} \mathrm{H}_{8} \mathrm{O}$ & $1120.21,3.600$ & 96.057 & 28861 & \\
\hline N-Methylene-tert-butylamine & $\mathrm{C}_{5} \mathrm{H}_{11} \mathrm{~N}$ & $1120.21,4.299$ & 85.0886 & 15479 & \\
\hline 2-Methylundecane & $\mathrm{C}_{12} \mathrm{H}_{26}$ & $1138.21,4.654$ & 170.2029 & 16146 & \\
\hline 6-Methylno hyphen-tridecane & $\mathrm{C}_{14} \mathrm{H}_{30}$ & $1144.21,4.356$ & 198.2342 & 335839 & \\
\hline $\begin{array}{l}\text { 2,2-Dimethyl-4-(methylethyl)-2H- } \\
\text { imidazole }\end{array}$ & $\mathrm{C}_{8} \mathrm{H}_{14} \mathrm{~N}_{2}$ & $1162.22,3.578$ & 138.1151 & 20608 & \\
\hline 4-Methyl-decane & $\mathrm{C}_{11} \mathrm{H}_{24}$ & $1192.23,4.382$ & 156.1873 & 509051 & \\
\hline 2-Methyldecane & $\mathrm{C}_{11} \mathrm{H}_{24}$ & $1198.23,4.423$ & 156.1873 & 600613 & \\
\hline Undecane & $\mathrm{C}_{11} \mathrm{H}_{24}$ & $1216.23,4.691$ & 156.1873 & 75699 & \\
\hline (1-Methylpropyl)cyclohexane & $\mathrm{C}_{10} \mathrm{H}_{20}$ & $1222.24,3.597$ & 140.156 & 22323 & \\
\hline 1-Methylaziridine & $\mathrm{C}_{3} \mathrm{H}_{7} \mathrm{~N}$ & $1222.24,4.672$ & 57.0573 & 69399 & \\
\hline 6-Ethyl-2-methyloctane & $\mathrm{C}_{11} \mathrm{H}_{24}$ & $1228.24,4.349$ & 156.1873 & 115214 & \\
\hline Butylcyclohexane & $\mathrm{C}_{10} \mathrm{H}_{20}$ & $1234.24,3.672$ & 140.156 & 425014 & \\
\hline 4,6-Dimethyldecan-1-ol & $\mathrm{C}_{12} \mathrm{H}_{26} \mathrm{O}$ ? & $1234.24,3.939$ & 186.1978 & 275040 & $3.74 \mathrm{E}-3$ \\
\hline 3-Methyldecane & $\mathrm{C}_{11} \mathrm{H}_{24}$ & $1234.24,4.392$ & 156.1873 & 614752 & \\
\hline 1-Ethyl-4-methylno hyphen-benzene & $\mathrm{C}_{9} \mathrm{H}_{12}$ & $1264.25,2.628$ & 120.0934 & 173809 & \\
\hline (2S,6R)-2,6-Dimethyl-1-octanol & $\mathrm{C}_{10} \mathrm{H}_{22} \mathrm{O}$ & $1276.25,3.956$ & 158.1665 & 267793 & \\
\hline 1,2,4-Trimethylbenzene & $\mathrm{C}_{9} \mathrm{H}_{12}$ & $1288.25,2.649$ & 120.0934 & 539832 & \\
\hline 3-Methyl-1-hexen-5-one & $\mathrm{C}_{7} \mathrm{H}_{12} \mathrm{O}$ & $1312.26,4.264$ & 112.0883 & 15677 & \\
\hline
\end{tabular}




\begin{tabular}{|c|c|c|c|c|c|}
\hline Hexadecane & $\mathrm{C}_{16} \mathrm{H}_{34}$ & $1348.27,4.642$ & 226.2655 & 136373 & \\
\hline Octacosane & $\mathrm{C}_{28} \mathrm{H}_{58}$ & $1372.28,4.647$ & 394.4533 & 47236 & \\
\hline (4RS,5S)-5-Methylhept-2-yn-4-ol & $\mathrm{C}_{8} \mathrm{H}_{14} \mathrm{O}$ & $1396.29,3.910$ & 126.1039 & 56414 & \\
\hline $\begin{array}{l}\text { 1-Isopropyl-1-methylno hyphen- } \\
\text { cyclohexane }\end{array}$ & $\mathrm{C}_{10} \mathrm{H}_{20}$ & $1420.29,3.970$ & 140.156 & 174765 & \\
\hline 3,3-Dimethylhexane & $\mathrm{C}_{8} \mathrm{H}_{18}$ & $1420.29,4.665$ & 114.1403 & 279770 & \\
\hline N-Methoxy-N-methylbenzamide & $\mathrm{C}_{9} \mathrm{H}_{11} \mathrm{NO}_{2}$ & $1438.3,2.783$ & 165.0784 & 10031 & \\
\hline 3,7-Dimethyldecane & $\mathrm{C}_{12} \mathrm{H}_{26}$ & $1450.3,4.608$ & 170.2029 & 345459 & \\
\hline trans-Decahydronaphthalene & $\mathrm{C}_{10} \mathrm{H}_{18}$ & $1468.31,3.327$ & 138.1403 & 131521 & \\
\hline (2-Methylbutyl)cyclohexane & $\mathrm{C}_{11} \mathrm{H}_{22}$ & $1474.31,3.929$ & 154.1716 & 282149 & \\
\hline Methyl(1-methylethyl)benzene & $\mathrm{C}_{10} \mathrm{H}_{14}$ & $1492.31,2.761$ & 134.109 & 65850 & \\
\hline 2-Methylheptadecane & $\mathrm{C}_{18} \mathrm{H}_{38}$ & $1510.32,4.589$ & 254.2968 & 148470 & \\
\hline 5-Ethyldecane & $\mathrm{C}_{12} \mathrm{H}_{26}$ & $1528.32,4.572$ & 170.2029 & 227256 & \\
\hline 3-Ethoxy-1-propyne & $\mathrm{C}_{5} \mathrm{H}_{8} \mathrm{O}$ & $1552.33,4.115$ & 84.057 & 28580 & \\
\hline 1-Ethyl-2,4-dimethylcyclohexane & $\mathrm{C}_{10} \mathrm{H}_{20}$ & $1570.34,4.199$ & 140.156 & 112395 & \\
\hline 2,6-Dimethylno hyphen-undecane & $\mathrm{C}_{13} \mathrm{H}_{28}$ & $1570.34,4.593$ & 184.2186 & 1129458 & \\
\hline 1,2,4-Trimethylbenzene & $\mathrm{C}_{9} \mathrm{H}_{12}$ & $1588.34,2.603$ & 120.0934 & 82582 & \\
\hline 1-Methyl-3-propylbenzene & $\mathrm{C}_{10} \mathrm{H}_{14}$ & $1624.35,2.782$ & 134.109 & 114077 & \\
\hline 4-Methyldodecane & $\mathrm{C}_{13} \mathrm{H}_{28}$ & $1630.35,4.535$ & 184.2186 & 97000 & \\
\hline Nonadecane & $\mathrm{C}_{19} \mathrm{H}_{40}$ & $1636.35,4.831$ & 268.3125 & 33266 & \\
\hline $\begin{array}{l}\text { 1-(2-Methylphenyl)-3-phenyl-2- } \\
\text { propanone }\end{array}$ & $\mathrm{C}_{16} \mathrm{H}_{16} \mathrm{O}$ ? & $1642.36,2.783$ & 224.1196 & 10836 & $1.47 \mathrm{E}-4$ \\
\hline 3-Methylundecane & $\mathrm{C}_{12} \mathrm{H}_{26}$ & $1642.36,4.572$ & 170.2029 & 675891 & \\
\hline Pentylcyclohexane & $\mathrm{C}_{11} \mathrm{H}_{22}$ & $1654.36,3.867$ & 154.1716 & 404304 & \\
\hline Octadecane & $\mathrm{C}_{18} \mathrm{H}_{38}$ & $1654.36,4.825$ & 254.2968 & 89841 & \\
\hline trans-2-Methyldecalin & $\mathrm{C}_{11} \mathrm{H}_{20}$ & $1660.36,3.538$ & 152.156 & 129156 & \\
\hline 1-Ethyl-2,4-dimethylbenzene & $\mathrm{C}_{10} \mathrm{H}_{14}$ & $1672.37,2.757$ & 134.109 & 259121 & \\
\hline 2-(1-Phenylethyl)phenol & $\mathrm{C}_{14} \mathrm{H}_{14} \mathrm{O}$ ? & $1708.38,4.808$ & 198.1039 & 63926 & $8.69 \mathrm{E}-4$ \\
\hline 7,9-Dimethylhexadecane & $\mathrm{C}_{18} \mathrm{H}_{38}$ & $1738.38,4.773$ & 254.2968 & 319480 & \\
\hline 4-(Allyloxy)-2-butyne & $\mathrm{C}_{7} \mathrm{H}_{10} \mathrm{O}$ & $1744.39,4.062$ & 110.0726 & 17546 & \\
\hline trans-4a-Methyldecahydronaphthalene & $\mathrm{C}_{11} \mathrm{H}_{20}$ & $1756.39,3.473$ & 152.156 & 51382 & \\
\hline
\end{tabular}




\begin{tabular}{|c|c|c|c|c|c|}
\hline 1,2-Dibutylcyclopropan & $\mathrm{C}_{11} \mathrm{H}_{22}$ & $1768.39,4.346$ & 154.1716 & 20883 & \\
\hline Pentadecane & $\mathrm{C}_{15} \mathrm{H}_{32}$ & $1768.39,4.746$ & 212.2499 & 97892 & \\
\hline Tridecane & $\mathrm{C}_{13} \mathrm{H}_{28}$ & $1774.39,4.536$ & 184.2186 & 4795319 & \\
\hline cis-1,2-Dibutylcyclopropane & $\mathrm{C}_{11} \mathrm{H}_{22}$ & $1786.4,4.053$ & 154.1716 & 15605 & \\
\hline (1-Methylbutyl)benzene & $\mathrm{C}_{11} \mathrm{H}_{16}$ & $1792.4,2.938$ & 148.1247 & 69884 & \\
\hline 1-Tridecyne & $\mathrm{C}_{13} \mathrm{H}_{24}$ & $1792.4,3.466$ & 180.1873 & 8999 & \\
\hline o-Cymene & $\mathrm{C}_{10} \mathrm{H}_{14}$ & $1816.41,2.726$ & 134.109 & 98098 & \\
\hline 1-Methyl-2-pentylcyclohexane & $\mathrm{C}_{12} \mathrm{H}_{24}$ & $1822.41,4.094$ & 168.1873 & 376000 & \\
\hline 1-Methyl-3-(1-methylethyl)benzene & $\mathrm{C}_{10} \mathrm{H}_{14}$ & $1828.41,2.881$ & 134.109 & 44118 & \\
\hline 9-Octadecenoic acid & $\mathrm{C}_{18} \mathrm{H}_{34} \mathrm{O}_{2}$ & $1870.42,3.968$ & 282.2553 & 8959 & \\
\hline 3-Methyldodecane & $\mathrm{C}_{13} \mathrm{H}_{28}$ & $1882.43,4.710$ & 184.2186 & 98229 & \\
\hline 3,4-Dimethylcumene & $\mathrm{C}_{11} \mathrm{H}_{16}$ & $1888.43,2.841$ & 148.1247 & 40425 & \\
\hline $\begin{array}{l}\text { 2-Methyl-2-methyl-2-propenyl } \\
\text { butanoate }\end{array}$ & $\mathrm{C}_{9} \mathrm{H}_{16} \mathrm{O}_{2}$ & $1900.43,4.645$ & 156.1145 & 38921 & \\
\hline (2S-trans)-3-Octyloxiranemethanol & $\mathrm{C}_{11} \mathrm{H}_{22} \mathrm{O}_{2} ?$ & $1918.44,4.295$ & 186.1614 & 15203 & $2.07 \mathrm{E}-4$ \\
\hline 2-Methyl-4-hexyn-3-ol & $\mathrm{C}_{7} \mathrm{H}_{12} \mathrm{O}$ & $1924.44,4.217$ & 112.0883 & 20883 & \\
\hline 2,6,10,15-Tetramethylheptadecane & $\mathrm{C}_{21} \mathrm{H}_{44}$ & $1936.44,4.927$ & 296.3438 & 314975 & \\
\hline 1,2,4,5-Tetramethylbenzene & $\mathrm{C}_{10} \mathrm{H}_{14}$ & $1960.45,2.746$ & 134.109 & 85776 & \\
\hline trans-1-Butyl-2-hexylcyclopropane & $\mathrm{C}_{13} \mathrm{H}_{26}$ & $1960.45,4.318$ & 182.2029 & 26925 & \\
\hline 6-Methyldodecane & $\mathrm{C}_{13} \mathrm{H}_{28}$ & $1972.45,4.639$ & 184.2186 & 840710 & \\
\hline 4-Methyl-1,6-heptadien-4-ol & $\mathrm{C}_{8} \mathrm{H}_{14} \mathrm{O}$ & $1972.45,4.910$ & 126.1039 & 20658 & \\
\hline 3-Ethyl-2,3-dihydro-1-benzofuran & $\mathrm{C}_{10} \mathrm{H}_{12} \mathrm{O}$ ? & $1984.45,2.864$ & 148.0883 & 16876 & $2.30 \mathrm{E}-4$ \\
\hline 2,4-Dimethylundecane & $\mathrm{C}_{13} \mathrm{H}_{28}$ & $1984.45,4.608$ & 184.2186 & 214732 & \\
\hline 1,2,3,4-Tetramethylbenzene & $\mathrm{C}_{10} \mathrm{H}_{14}$ & $1996.46,2.710$ & 134.109 & 130414 & \\
\hline 2,3,7-Trimethyloctane & $\mathrm{C}_{11} \mathrm{H}_{24}$ & $2008.46,4.906$ & 156.1873 & 986150 & \\
\hline 2-Methyl-2-dicyanomethyladamantane & $\mathrm{C}_{14} \mathrm{H}_{18} \mathrm{~N}_{2}$ & $2014.46,3.390$ & 214.1465 & 12653 & \\
\hline 2-Methyl-dodecane & $\mathrm{C}_{13} \mathrm{H}_{28}$ & $2014.46,4.644$ & 184.2186 & 659951 & \\
\hline 1-Methyl-4-(1-methylpropyl)benzene & $\mathrm{C}_{11} \mathrm{H}_{16}$ & $2020.47,2.854$ & 148.1247 & 120308 & \\
\hline (E)-1-Cyclohexyl-1-hexene & $\mathrm{C}_{12} \mathrm{H}_{22}$ & $2026.47,3.333$ & 166.1716 & 12993 & \\
\hline Hexylcyclohexane & $\mathrm{C}_{12} \mathrm{H}_{24}$ & $2086.48,3.914$ & 168.1873 & 464435 & \\
\hline
\end{tabular}




\begin{tabular}{|c|c|c|c|c|c|}
\hline 1-(4-Methylphenyl)-1-propanone & $\mathrm{C}_{10} \mathrm{H}_{12} \mathrm{O}$ & $2098.49,2.853$ & 148.0883 & 39592 & \\
\hline 3-Cyclohexylcyclohexanone & $\mathrm{C}_{12} \mathrm{H}_{20} \mathrm{O}$ & $2104.49,4.165$ & 180.1509 & 67775 & \\
\hline 1-Methyl-2-(1-methylethyl)benzene & $\mathrm{C}_{10} \mathrm{H}_{14}$ & $2128.5,2.835$ & 134.109 & 40262 & \\
\hline $\begin{array}{l}\text { (4R,5S,8R)-5-Isopropyl-8-methyl-1- } \\
\text { oxaspiro[3.5]nonane }\end{array}$ & $\mathrm{C}_{12} \mathrm{H}_{22} \mathrm{O}$ ? & $2128.5,4.173$ & 182.1665 & 14706 & $2.00 \mathrm{E}-4$ \\
\hline 2,6,10,14-Tetramethylhexadecane & $\mathrm{C}_{20} \mathrm{H}_{42}$ & $2164.51,4.718$ & 282.3281 & 281089 & \\
\hline (1,1-Dimethylbutyl)benzene & $\mathrm{C}_{12} \mathrm{H}_{18}$ & $2170.51,2.973$ & 162.1403 & 54617 & \\
\hline $\begin{array}{l}\text { 4-Methyl-1-[4-(1-methylethenyl)-1- } \\
\text { cyclohexen-1-yl]-3-penten-1-one }\end{array}$ & $\mathrm{C}_{15} \mathrm{H}_{22} \mathrm{O}$ ? & $2200.52,3.260$ & 218.1665 & 23844 & \\
\hline Heptadecane & $\mathrm{C}_{17} \mathrm{H}_{36}$ & $2224.52,4.690$ & 240.2812 & 740136 & \\
\hline $\begin{array}{l}\text { 1-Ethenyl-5-(1-methylethylidene)- 1,3- } \\
\text { cyclopentadiene }\end{array}$ & $\mathrm{C}_{10} \mathrm{H}_{12}$ & $2230.53,2.555$ & 132.0934 & 10185 & \\
\hline $\begin{array}{l}\text { 1-(1,1-Dimethylethyl)-4-methyl- } \\
\text { benzene }\end{array}$ & $\mathrm{C}_{11} \mathrm{H}_{16}$ & $2308.55,2.768$ & 148.1247 & 27447 & \\
\hline $\begin{array}{l}\text { 1-Methyl-3-(2-methylpropyl)- } \\
\text { cyclopentane }\end{array}$ & $\mathrm{C}_{10} \mathrm{H}_{20}$ & $2308.55,4.024$ & 140.156 & 126700 & \\
\hline 1,2,3,4-Tetrahydronaphthalene & $\mathrm{C}_{10} \mathrm{H}_{12}$ & $2332.55,2.473$ & 132.0934 & 18659 & \\
\hline 1-Ethyl-4-(1-methylethyl)benzene & $\mathrm{C}_{11} \mathrm{H}_{16}$ & $2332.55,2.729$ & 148.1247 & 18739 & \\
\hline $\begin{array}{l}\text { 1-Methyl-3-(1-methylethyl)- } \\
\text { cyclohexane }\end{array}$ & $\mathrm{C}_{10} \mathrm{H}_{20}$ & $2332.55,3.995$ & 140.156 & 71111 & \\
\hline Hexacosane & $\mathrm{C}_{26} \mathrm{H}_{54}$ & $2350.56,4.608$ & 366.422 & 76370 & \\
\hline Ethyl-1,2,4-trimethylbenzene & $\mathrm{C}_{11} \mathrm{H}_{16}$ & $2380.57,2.718$ & 148.1247 & 19923 & \\
\hline 5-Methyltridecane & $\mathrm{C}_{14} \mathrm{H}_{30}$ & $2380.57,4.587$ & 198.2342 & 361510 & \\
\hline 3,3,5-Trimethylheptane & $\mathrm{C}_{10} \mathrm{H}_{22}$ & $2380.57,4.814$ & 142.1716 & 171053 & \\
\hline Tetracosane & $\mathrm{C}_{24} \mathrm{H}_{50}$ & $2392.57,4.832$ & 338.3907 & 38905 & \\
\hline 4-Methyltridecane & $\mathrm{C}_{14} \mathrm{H}_{30}$ & $2398.57,4.587$ & 198.2342 & 366109 & \\
\hline 2,6,11-Trimethyldodecane & $\mathrm{C}_{15} \mathrm{H}_{32}$ & $2404.58,4.824$ & 212.2499 & 244147 & \\
\hline 2-Methyltridecane & $\mathrm{C}_{14} \mathrm{H}_{30}$ & $2416.58,4.587$ & 198.2342 & 664095 & \\
\hline 1,17-Octadecadiene & $\mathrm{C}_{18} \mathrm{H}_{34}$ & $2458.59,4.170$ & 250.2655 & 50087 & \\
\hline 3-Methyltridecane, & $\mathrm{C}_{14} \mathrm{H}_{30}$ & $2458.59,4.531$ & 198.2342 & 649109 & \\
\hline $\begin{array}{l}\text { 2-(1,1-Dimethylethyl)-1,2,3,4,5,6,7,8- } \\
\text { octahydronaphthalene }\end{array}$ & $\mathrm{C}_{14} \mathrm{H}_{24}$ & $2464.59,3.170$ & 192.1873 & 14212 & \\
\hline $\begin{array}{l}\text { 1,4-Dimethyl-2-(2- } \\
\text { methylpropyl)benzene }\end{array}$ & $\mathrm{C}_{12} \mathrm{H}_{18}$ & $2596.63,2.835$ & 162.1403 & 28678 & \\
\hline m-Menthane & $\mathrm{C}_{10} \mathrm{H}_{20}$ & $2656.65,4.058$ & 140.156 & 322420 & \\
\hline (4E)-2,2,4-Trimethylhex-4-en-3-one & $\mathrm{C}_{9} \mathrm{H}_{16} \mathrm{O}$ & $2668.65,4.200$ & 140.1196 & 19002 & \\
\hline $\begin{array}{l}\text { trans-1-Ethyl-1,4-dimethylno space } \\
\text { cyclohexane }\end{array}$ & $\mathrm{C}_{10} \mathrm{H}_{20}$ & $2674.65,4.047$ & 140.156 & 105327 & \\
\hline
\end{tabular}




\begin{tabular}{|c|c|c|c|c|c|}
\hline 1-Methyl-2-octylcyclopropane & $\mathrm{C}_{12} \mathrm{H}_{24}$ & $2698.66,4.059$ & 168.1873 & 20192 & \\
\hline trans-1-methyl-2-nonylcyclohexane & $\mathrm{C}_{16} \mathrm{H}_{32}$ & $2734.67,3.977$ & 224.2499 & 51373 & \\
\hline cis-bicyclo[3.3.0] octan-1-ol & $\mathrm{C}_{8} \mathrm{H}_{14} \mathrm{O}$ & $2740.67,3.954$ & 126.1039 & 28849 & \\
\hline 1,2,3,4-Tetrahydro-6-methylnaphthalene & $\mathrm{C}_{11} \mathrm{H}_{14}$ & $2746.67,2.526$ & 146.109 & 50083 & \\
\hline 2,3,4,5-Tetrahydro-6-hexylpyridine & $\mathrm{C}_{11} \mathrm{H}_{21} \mathrm{~N}$ & $2746.67,3.933$ & 167.1669 & 31626 & \\
\hline 7-Methylpentadecane & $\mathrm{C}_{16} \mathrm{H}_{34}$ & $2746.67,4.536$ & 226.2655 & 792788 & \\
\hline 2,6,10,14-Tetramethylheptadecane & $\mathrm{C}_{21} \mathrm{H}_{44}$ & $2752.68,4.762$ & 296.3438 & 955975 & \\
\hline $\begin{array}{l}\text { 2,3-Dihydro-1,4,7-trimethylno space } \\
\text { indene }\end{array}$ & $\mathrm{C}_{12} \mathrm{H}_{16}$ & $2764.68,2.684$ & 160.1247 & 26478 & \\
\hline (S)-Undec-1-en-4-ol & $\mathrm{C}_{11} \mathrm{H}_{22} \mathrm{O}$ ? & $2764.68,4.180$ & 170.1665 & 26092 & $3.55 \mathrm{E}-4$ \\
\hline 5-Methyltetradecane & $\mathrm{C}_{15} \mathrm{H}_{32}$ & $2764.68,4.505$ & 212.2499 & 309867 & \\
\hline Octyl pentyl ether & $\mathrm{C}_{13} \mathrm{H}_{28} \mathrm{O}$ ? & $2776.68,4.488$ & 200.2135 & 29603 & 4.03E-4 \\
\hline 1-Ethyl-1,3-dimethylcyclohexane & $\mathrm{C}_{10} \mathrm{H}_{20}$ & $2782.68,4.200$ & 140.156 & 56781 & \\
\hline 4-Methylno space tetradecane & $\mathrm{C}_{15} \mathrm{H}_{32}$ & $2782.68,4.515$ & 212.2499 & 315210 & \\
\hline cis-1-Butyl-2-undecylcyclopropane & $\mathrm{C}_{18} \mathrm{H}_{36}$ & $2806.69,4.160$ & 252.2812 & 68202 & \\
\hline $\begin{array}{l}\text { 1-Isopropyl-8-methyl-1,2,3,4- } \\
\text { tetrahydronaphthalene }\end{array}$ & $\mathrm{C}_{14} \mathrm{H}_{20}$ & $2818.69,2.659$ & 188.156 & 9390 & \\
\hline 2,6-Dimethylheptadecane & $\mathrm{C}_{19} \mathrm{H}_{40}$ & $2836.7,4.485$ & 268.3125 & 530222 & \\
\hline $\begin{array}{l}\text { 1,2,3,4-Tetrahydro-2,7- } \\
\text { dimethylnaphthalene }\end{array}$ & $\mathrm{C}_{12} \mathrm{H}_{16}$ & $2902.72,2.627$ & 160.1247 & 45797 & \\
\hline 1,2,3,4-Tetrahydro-5-methylnaphthalene & $\mathrm{C}_{11} \mathrm{H}_{14}$ & $2908.72,2.444$ & 146.109 & 19204 & \\
\hline Octylcyclohexane & $\mathrm{C}_{14} \mathrm{H}_{28}$ & $2908.72,3.862$ & 196.2186 & 532023 & \\
\hline 2-Methylnonane & $\mathrm{C}_{10} \mathrm{H}_{22}$ & $2920.72,4.614$ & 142.1716 & 66665 & \\
\hline 1,3-Dimethyl-1H-Inden-1-ol & $\mathrm{C}_{11} \mathrm{H}_{12} \mathrm{O}$ ? & $2938.73,2.607$ & 160.0883 & 12095 & $1.65 \mathrm{E}-4$ \\
\hline (Z/E)-2,2,4,5-Tetramethyl-3-heptene & $\mathrm{C}_{11} \mathrm{H}_{22}$ & $2944.73,4.289$ & 154.1716 & 22228 & \\
\hline 1-Hexadecene & $\mathrm{C}_{16} \mathrm{H}_{32}$ & $3016.75,4.041$ & 224.2499 & 48889 & \\
\hline 1-Methylnaphthalene & $\mathrm{C}_{11} \mathrm{H}_{10}$ & $3022.75,2.318$ & 142.0777 & 35221 & \\
\hline $\begin{array}{l}\text { 1,1'-(1,2-Dimethyl-1,2-ethanediyl)bis- } \\
\text { cyclohexane }\end{array}$ & $\mathrm{C}_{16} \mathrm{H}_{30}$ & $3064.76,3.981$ & 222.2342 & 113425 & \\
\hline 3-Acetyl-3-methylcyclohexanone & $\mathrm{C}_{9} \mathrm{H}_{14} \mathrm{O}_{2} ?$ & $3112.78,4.190$ & 154.0988 & 24500 & 3.33E-4 \\
\hline 1-Cyclopentylidene-2,4-cyclopentadiene & $\mathrm{C}_{10} \mathrm{H}_{12}$ & $3118.78,2.526$ & 132.0934 & 9242 & \\
\hline 5-Phenylpent-1-en-3-yne & $\mathrm{C}_{11} \mathrm{H}_{10}$ & $3124.78,2.275$ & 142.0777 & 15453 & \\
\hline 4-Methylpentadecane, & $\mathrm{C}_{16} \mathrm{H}_{34}$ & $3148.79,4.438$ & 226.2655 & 329068 & \\
\hline
\end{tabular}




\begin{tabular}{|c|c|c|c|c|c|}
\hline 2-Methylnonadecane & $\mathrm{C}_{20} \mathrm{H}_{42}$ & $3166.79,4.449$ & 282.3281 & 527385 & \\
\hline 3-Isoxazolepropanol & $\mathrm{C}_{6} \mathrm{H}_{9} \mathrm{NO}_{2} ?$ & $3178.8,4.124$ & 127.0628 & 21917 & $2.98 \mathrm{E}-4$ \\
\hline 4,4,7,7-Tetramethyl-1,9-decadiene & $\mathrm{C}_{14} \mathrm{H}_{26}$ & $3190.8,4.084$ & 194.2029 & 34967 & \\
\hline 2,6,10-Trimethyltridecane & $\mathrm{C}_{16} \mathrm{H}_{34}$ & $3190.8,4.383$ & 226.2655 & 31109 & \\
\hline 2,5,9-Trimethyldecane & $\mathrm{C}_{13} \mathrm{H}_{28}$ & $3334.84,4.681$ & 184.2186 & 42626 & \\
\hline 5-Methyl-5-hexen-2-one & $\mathrm{C}_{7} \mathrm{H}_{12} \mathrm{O}$ ? & $3352.85,3.954$ & 112.0883 & 22323 & 3.04E-4 \\
\hline $\begin{array}{l}\text { 1,2,3,4-Tetrahydro-2,5,7-trimethyl- } \\
\text { naphthalene }\end{array}$ & $\mathrm{C}_{13} \mathrm{H}_{18}$ & $3394.86,2.627$ & 174.1403 & 9005 & \\
\hline 1,7-Dimethylnaphthalene & $\mathrm{C}_{12} \mathrm{H}_{12}$ & $3418.87,2.382$ & 156.0934 & 66766 & \\
\hline (E)-2-Tetradecen-1-ol & $\mathrm{C}_{14} \mathrm{H}_{28} \mathrm{O}$ ? & $3424.87,3.942$ & 212.2135 & 15851 & $2.16 \mathrm{E}-4$ \\
\hline Tetradecylcyclooctane & $\mathrm{C}_{22} \mathrm{H}_{44}$ & $3430.87,3.957$ & 308.3438 & 74223 & \\
\hline 2,2,3,3-Tetramethylhexane & $\mathrm{C}_{10} \mathrm{H}_{22}$ & $3478.88,4.382$ & 142.1716 & 240297 & \\
\hline 4-tert-Butylhex-1-ene & $\mathrm{C}_{10} \mathrm{H}_{20}$ & $3490.89,4.597$ & 140.156 & 67205 & \\
\hline 4-Methylhexadecane & $\mathrm{C}_{17} \mathrm{H}_{36}$ & $3496.89,4.402$ & 240.2812 & 217190 & \\
\hline 1,4-Dimethylnaphthalene & $\mathrm{C}_{12} \mathrm{H}_{12}$ & $3502.89,2.345$ & 156.0934 & 19634 & \\
\hline 1,5-Dimethylnaphthalene & $\mathrm{C}_{12} \mathrm{H}_{12}$ & $3520.9,2.325$ & 156.0934 & 59882 & \\
\hline 1-Octyl-1,4-dihydronaphthalene & $\mathrm{C}_{18} \mathrm{H}_{26}$ & $3520.9,2.465$ & 242.2029 & 9368 & \\
\hline 3-Methylhexadecane & $\mathrm{C}_{17} \mathrm{H}_{36}$ & $3550.9,4.342$ & 240.2812 & 378860 & \\
\hline 2-Methylhexadecane & $\mathrm{C}_{17} \mathrm{H}_{36}$ & $3610.92,4.714$ & 240.2812 & 237185 & \\
\hline Dotriacontane & $\mathrm{C}_{32} \mathrm{H}_{66}$ & $3634.93,4.468$ & 450.5159 & 99582 & \\
\hline $\begin{array}{l}\text { 7- } \\
\text { Phenyltetracyclo[4.1.0.02,4.03,5]heptane }\end{array}$ & $\mathrm{C}_{13} \mathrm{H}_{12}$ & $3772.97,2.272$ & 168.0934 & 10836 & \\
\hline 5-Methyldodecane & $\mathrm{C}_{13} \mathrm{H}_{28}$ & $3808.98,4.340$ & 184.2186 & 178189 & \\
\hline 2-Methyleicosane & $\mathrm{C}_{21} \mathrm{H}_{44}$ & $3838.99,4.346$ & 296.3438 & 377357 & \\
\hline 1,6,7-Trimethyl-naphthalene & $\mathrm{C}_{13} \mathrm{H}_{14}$ & $3869,2.382$ & 170.109 & 25816 & \\
\hline 1,4,6-Trimethylnaphthalene & $\mathrm{C}_{13} \mathrm{H}_{14}$ & $3881,2.399$ & 170.109 & 31007 & \\
\hline 3,7-Dimethylnonane & $\mathrm{C}_{11} \mathrm{H}_{24}$ & $3881,4.315$ & 156.1873 & 283347 & \\
\hline 2,5-Dimethylheptane & $\mathrm{C}_{9} \mathrm{H}_{20}$ & $3887,4.636$ & 128.156 & 26935 & \\
\hline 2,6,10-Trimethyltridecane & $\mathrm{C}_{16} \mathrm{H}_{34}$ & $3917.01,4.652$ & 226.2655 & 34758 & \\
\hline 4,4-Dimethyloctane & $\mathrm{C}_{10} \mathrm{H}_{22}$ & $3923.01,4.431$ & 142.1716 & 32462 & \\
\hline 2-Pyrrolidinone & $\mathrm{C}_{4} \mathrm{H}_{7} \mathrm{NO} ?$ & $3923.01,4.604$ & 85.0522 & 13831 & $1.88 \mathrm{E}-4$ \\
\hline
\end{tabular}




\begin{tabular}{|c|c|c|c|c|c|}
\hline 2,3-Octanedione & $\mathrm{C}_{8} \mathrm{H}_{14} \mathrm{O}_{2}$ ? & $3971.02,4.538$ & 142.0988 & 17010 & $2.31 \mathrm{E}-4$ \\
\hline 1,4,5-Trimethylnaphthalene & $\mathrm{C}_{13} \mathrm{H}_{14}$ & $3977.03,2.349$ & 170.109 & 7427 & \\
\hline 2,2,3,3-Tetramethylbutane & $\mathrm{C}_{8} \mathrm{H}_{18}$ & $3989.03,4.412$ & 114.1403 & 65117 & \\
\hline Tricosane & $\mathrm{C}_{23} \mathrm{H}_{48}$ & $4031.04,4.520$ & 324.3751 & 117734 & \\
\hline 10-Methylnonadecane & $\mathrm{C}_{20} \mathrm{H}_{42}$ & $4091.06,4.330$ & 282.3281 & 371382 & \\
\hline (Z)-2,2,3,5,5-pentamethyl-3-hexene & $\mathrm{C}_{11} \mathrm{H}_{22}$ & $4145.07,4.013$ & 154.1716 & 45891 & \\
\hline Eicosane & $\mathrm{C}_{20} \mathrm{H}_{42}$ & $4145.07,4.289$ & 282.3281 & 165528 & \\
\hline $\begin{array}{l}\text { 1-Methyl-4-(phenylmethyl)no hyphen } \\
\text { benzene }\end{array}$ & $\mathrm{C}_{14} \mathrm{H}_{14}$ & $4163.08,2.307$ & 182.109 & 9687 & \\
\hline 2,2,4-Trimethyl-2H-imidazole & $\mathrm{C}_{6} \mathrm{H}_{10} \mathrm{~N}_{2}$ & $4175.08,3.873$ & 110.0838 & 14821 & \\
\hline Heptacosane & $\mathrm{C}_{27} \mathrm{H}_{56}$ & $4193.09,4.253$ & 380.4377 & 195118 & \\
\hline $2,2,3,3,4,4,5,5$-Octamethylhexane & $\mathrm{C}_{14} \mathrm{H}_{30}$ & $4211.09,4.356$ & 198.2342 & 40228 & \\
\hline Undecylcyclohexane & $\mathrm{C}_{17} \mathrm{H}_{34}$ & $4319.12,3.749$ & 238.2655 & 212672 & \\
\hline Allyl octyl ether & $\mathrm{C}_{11} \mathrm{H}_{22} \mathrm{O} ?$ & $4349.13,4.272$ & 170.1665 & 26100 & $3.55 \mathrm{E}-4$ \\
\hline (E)-9-Tricosene & $\mathrm{C}_{23} \mathrm{H}_{46}$ & $4367.14,3.919$ & 322.3594 & 39654 & \\
\hline 1-Hexadecanol & $\mathrm{C}_{16} \mathrm{H}_{34} \mathrm{O} ?$ & $4379.14,3.937$ & 242.2604 & 30034 & $4.09 \mathrm{E}-4$ \\
\hline 2,6,10-Trimethylpentadecane & $\mathrm{C}_{18} \mathrm{H}_{38}$ & $4409.15,4.281$ & 254.2968 & 112636 & \\
\hline Tetratetracontane & $\mathrm{C}_{44} \mathrm{H}_{90}$ & $4673.23,4.263$ & 618.7037 & 472034 & \\
\hline Docosane & $\mathrm{C}_{22} \mathrm{H}_{46}$ & $4949.31,4.227$ & 310.3594 & 371934 & \\
\hline 2,9-Dimethyldecane & $\mathrm{C}_{12} \mathrm{H}_{26}$ & $5015.32,4.243$ & 170.2029 & 204263 & \\
\hline (1-Methylethyl)cyclohexane & $\mathrm{C}_{9} \mathrm{H}_{18}$ & $5471.46,3.758$ & 126.1403 & 65691 & \\
\hline (S)-3,5-Dimethyl-hexan-1-ol & $\mathrm{C}_{8} \mathrm{H}_{18} \mathrm{O}$ ? & $5525.47,4.222$ & 130.1352 & 121871 & $1.65 \mathrm{E}-3$ \\
\hline 2,2-Dimethylpentane & $\mathrm{C}_{7} \mathrm{H}_{16}$ & $5561.48,4.193$ & 100.1247 & 54317 & \\
\hline 2-Methylene-3-butylamine & $\mathrm{C}_{5} \mathrm{H}_{9} \mathrm{~N}$ & $5975.6,3.772$ & 83.073 & 39535 & \\
\hline 2,2-Dimethylbutane & $\mathrm{C}_{6} \mathrm{H}_{14}$ & $6035.62,4.200$ & 86.109 & 42062 & \\
\hline $\begin{array}{l}\text { (3R)-3-Phenyl-2,3-dihydro-1H-isoindol- } \\
\text { 1-one }\end{array}$ & $\mathrm{C}_{14} \mathrm{H}_{11} \mathrm{NO}$ ? & $6155.65,1.101$ & 209.0835 & 64129 & $8.72 \mathrm{E}-4$ \\
\hline Pivalic acid & $\mathrm{C}_{5} \mathrm{H}_{10} \mathrm{O}_{2} ?$ & $6443.73,4.263$ & 102.0675 & 17759 & $2.42 \mathrm{E}-4$ \\
\hline
\end{tabular}


Table 3S18. Data obtained from the distillate fraction of condensate-like oil 3 by using GCxGC/(+EI)-TOF MS. Data provided are based on ChromaTOF software prediction of the molecular ion $\mathrm{m} / \mathrm{z}$ value and the identification of the compound by using the EI mass spectral library match using a match factor threshold of 800. Formulas with a question mark "?" might not have been accurately identified. The ratio of the peak area of each of these questionable compounds relative to all peak areas of the detected compounds is provided in the table below

\begin{tabular}{|c|c|c|c|c|c|}
\hline Name & Formula & $\begin{array}{c}\text { Retention Times (s) on } \\
\text { the Primary, Secondary } \\
\text { Column }\end{array}$ & $\begin{array}{l}\text { Molecular } \\
\text { Ion } \mathbf{m} / \mathbf{z}\end{array}$ & $\begin{array}{c}\text { Chromatographic } \\
\text { Peak Area }\end{array}$ & $\begin{array}{c}\text { Ratio } \\
\text { (Peak } \\
\text { area/total sum } \\
\text { of peak areas) } \\
\end{array}$ \\
\hline 3-Methylheptane & $\mathrm{C}_{8} \mathrm{H}_{18}$ & $406.002,2.407$ & 114.1403 & 341309 & \\
\hline 1,1,2-Trimethylcyclopentane & $\mathrm{C}_{8} \mathrm{H}_{16}$ & $424.007,2.310$ & 112.1247 & 93151 & \\
\hline 1,5-Octadien-3-ol & $\mathrm{C}_{8} \mathrm{H}_{14} \mathrm{O}$ & $436.01,2.381$ & 126.1039 & 28241 & \\
\hline Octane & $\mathrm{C}_{8} \mathrm{H}_{18}$ & $442.012,2.602$ & 114.1403 & 977057 & \\
\hline cis-1,3-Dimethylcyclohexane & $\mathrm{C}_{8} \mathrm{H}_{16}$ & $454.016,2.391$ & 112.1247 & 2908557 & \\
\hline Hexane & $\mathrm{C}_{6} \mathrm{H}_{14}$ & $460.017,2.798$ & 86.109 & 259659 & \\
\hline 2,4-Dimethylheptane & $\mathrm{C}_{9} \mathrm{H}_{20}$ & $466.019,2.818$ & 128.156 & 120996 & \\
\hline 1,1-Dimethylcyclohexane & $\mathrm{C}_{8} \mathrm{H}_{16}$ & $478.022,2.427$ & 112.1247 & 477175 & \\
\hline (E)-2-Hexenal & $\mathrm{C}_{6} \mathrm{H}_{10} \mathrm{O}$ & $478.022,2.671$ & 98.0726 & 12733 & \\
\hline 2,6-Dimethylheptane & $\mathrm{C}_{9} \mathrm{H}_{20}$ & $478.022,2.875$ & 128.156 & 517179 & \\
\hline trans-1,2-Dimethylcyclohexane & $\mathrm{C}_{8} \mathrm{H}_{16}$ & $496.028,2.504$ & 112.1247 & 699114 & \\
\hline 2,5-Dimethylheptane & $\mathrm{C}_{9} \mathrm{H}_{20}$ & $496.028,2.921$ & 128.156 & 1373200 & \\
\hline 1,4-Dimethylcyclohexane & $\mathrm{C}_{8} \mathrm{H}_{16}$ & $514.033,2.541$ & 112.1247 & 723005 & \\
\hline Piperidine & $\mathrm{C}_{5} \mathrm{H}_{11} \mathrm{~N}$ & $532.038,3.014$ & 85.0886 & 37268 & \\
\hline $\begin{array}{l}\text { 1,3,5-Trimethylno } \\
\text { hyphencyclohexane }\end{array}$ & $\mathrm{C}_{9} \mathrm{H}_{18}$ & $538.04,2.782$ & 126.1403 & 494101 & \\
\hline 1-Bicyclo[2.2.2]octanol & $\mathrm{C}_{8} \mathrm{H}_{14} \mathrm{O}$ & $550.043,2.839$ & 126.1039 & 27949 & \\
\hline 5-Methyldecane & $\mathrm{C}_{11} \mathrm{H}_{24}$ & $550.043,3.102$ & 156.1873 & 164858 & \\
\hline 1,1,3-Trimethylcyclohexane & $\mathrm{C}_{9} \mathrm{H}_{18}$ & $562.047,2.808$ & 126.1403 & 1100353 & \\
\hline $\begin{array}{l}\text { N-(But-3'-enyl)-N- } \\
\text { vinylformamide }\end{array}$ & $\mathrm{C}_{7} \mathrm{H}_{11} \mathrm{NO}$ & $574.05,2.926$ & 125.0835 & 5589 & \\
\hline 3-Methyloctane & $\mathrm{C}_{9} \mathrm{H}_{20}$ & $580.052,3.194$ & 128.156 & 2025199 & \\
\hline
\end{tabular}




\begin{tabular}{|c|c|c|c|c|c|}
\hline Ethylcyclohexane & $\mathrm{C}_{8} \mathrm{H}_{16}$ & $586.053,2.695$ & 112.1247 & 874266 & \\
\hline 1,2,4-Trimethylcyclohexane & $\mathrm{C}_{9} \mathrm{H}_{18}$ & $592.055,2.916$ & 126.1403 & 850858 & \\
\hline Toluene & $\mathrm{C}_{7} \mathrm{H}_{8}$ & $598.057,2.062$ & 92.0621 & 173979 & \\
\hline Nonane & $\mathrm{C}_{9} \mathrm{H}_{20}$ & $658.074,3.394$ & 128.156 & 2069420 & \\
\hline 1,2,3-Trimethylcyclohexane & $\mathrm{C}_{9} \mathrm{H}_{18}$ & $670.078,3.029$ & 126.1403 & 339821 & \\
\hline 2,4,6-Trimethylheptane & $\mathrm{C}_{10} \mathrm{H}_{22}$ & $682.081,3.636$ & 142.1716 & 589668 & \\
\hline 1-Ethyl-4-methylcyclohexane & $\mathrm{C}_{9} \mathrm{H}_{18}$ & $706.088,3.096$ & 126.1403 & 1443674 & \\
\hline 3,5-Dimethyloctane & $\mathrm{C}_{10} \mathrm{H}_{22}$ & $706.088,3.677$ & 142.1716 & 1195770 & \\
\hline $\begin{array}{l}\text { cis- } 1,1,3,5- \\
\text { Tetramethylcyclohexane }\end{array}$ & $\mathrm{C}_{10} \mathrm{H}_{20}$ & $718.091,3.343$ & 140.156 & 198005 & \\
\hline 2,6-Dimethyloctane & $\mathrm{C}_{10} \mathrm{H}_{22}$ & $736.096,3.734$ & 142.1716 & 1060512 & \\
\hline Octahydropentalene & $\mathrm{C}_{8} \mathrm{H}_{14}$ & $754.102,2.743$ & 110.109 & 64666 & \\
\hline 3,6-Dimethyloctane & $\mathrm{C}_{10} \mathrm{H}_{22}$ & $760.103,3.759$ & 142.1716 & 592477 & \\
\hline cis-1-Ethyl-2-methylcyclohexane & $\mathrm{C}_{9} \mathrm{H}_{18}$ & $778.109,3.168$ & 126.1403 & 672298 & \\
\hline $\begin{array}{l}\text { 3-Butyl-3,5- } \\
\text { dimethylcyclohexanone }\end{array}$ & $\mathrm{C}_{12} \mathrm{H}_{22} \mathrm{O}$ ? & $778.109,3.441$ & 182.1665 & 29229 & $3.70 \mathrm{E}-4$ \\
\hline 3-Hepten-2-one & $\mathrm{C}_{7} \mathrm{H}_{12} \mathrm{O}$ & $778.109,3.555$ & 112.0883 & 10166 & \\
\hline 2,3,3-Trimethyl-(Z)-4-nonene & $\mathrm{C}_{12} \mathrm{H}_{24}$ & $796.114,3.432$ & 168.1873 & 67068 & \\
\hline 1-Ethyl-1-methylcyclohexane & $\mathrm{C}_{9} \mathrm{H}_{18}$ & $808.117,3.142$ & 126.1403 & 105888 & \\
\hline $\begin{array}{l}(+)-(\mathrm{S})-1-B u t y l-2- \\
\text { methylenecyclopentane }\end{array}$ & $\mathrm{C}_{10} \mathrm{H}_{18}$ & $814.119,2.725$ & 138.1403 & 18401 & \\
\hline 4-Ethyloctane & $\mathrm{C}_{10} \mathrm{H}_{22}$ & $814.119,3.847$ & 142.1716 & 824269 & \\
\hline $\begin{array}{l}\text { 1,1,3,5-Tetramethyl- } \\
\text { transcyclohexane }\end{array}$ & $\mathrm{C}_{10} \mathrm{H}_{20}$ & $820.121,3.409$ & 140.156 & 109514 & \\
\hline $\begin{array}{l}\text { 2,4-Bis(methylethyl)-2-methyl- } \\
\text { 2,5-dihydro-1H-imidazole }\end{array}$ & $\mathrm{C}_{10} \mathrm{H}_{20} \mathrm{~N}_{2}$ & $832.124,3.389$ & 168.1621 & 10555 & \\
\hline 4-Methylnonane & $\mathrm{C}_{10} \mathrm{H}_{22}$ & $832.124,3.909$ & 142.1716 & 1928891 & \\
\hline $\begin{array}{l}\text { trans-1-Ethyl-1,3-dimethyl } \\
\text { cyclohexane }\end{array}$ & $\mathrm{C}_{10} \mathrm{H}_{20}$ & $838.126,3.492$ & 140.156 & 405342 & \\
\hline $\begin{array}{l}\text { 1-Cyano-2,6-dimethyl-1,5- } \\
\text { heptadien-3-ol }\end{array}$ & $\mathrm{C}_{10} \mathrm{H}_{15} \mathrm{NO} ?$ & $844.127,3.552$ & 165.1148 & 3422 & 4.33E-5 \\
\hline $\begin{array}{l}\text { 3-Methylene-1- } \\
\text { methylcycloheptane-1,2-diol }\end{array}$ & $\mathrm{C}_{9} \mathrm{H}_{16} \mathrm{O}_{2}$ ? & $850.129,3.227$ & 156.1145 & 24921 & $3.15 \mathrm{E}-4$ \\
\hline Propylcyclohexane & $\mathrm{C}_{9} \mathrm{H}_{18}$ & $856.131,3.276$ & 126.1403 & 538881 & \\
\hline 2,4-Dimethylpentane & $\mathrm{C}_{7} \mathrm{H}_{16}$ & $856.131,4.210$ & 100.1247 & 71555 & \\
\hline 7-Methyltridecane & $\mathrm{C}_{14} \mathrm{H}_{30}$ & $868.134,3.939$ & 198.2342 & 1031051 & \\
\hline cis-Octahydro-1H-indene & $\mathrm{C}_{9} \mathrm{H}_{16}$ & $874.136,3.088$ & 124.1247 & 106743 & \\
\hline
\end{tabular}




\begin{tabular}{|c|c|c|c|c|c|}
\hline $\begin{array}{l}\text { 3,7,7-Trimethyl-1,3,8-nonatrien- } \\
\text { 5-one }\end{array}$ & $\mathrm{C}_{12} \mathrm{H}_{18} \mathrm{O} ?$ & $874.136,3.383$ & 178.1352 & 8470 & $1.07 \mathrm{E}-4$ \\
\hline $\begin{array}{l}\text { (4E)-4- } \\
\text { Ethylidenebicyclo[3.1.0]hex-2- } \\
\text { ene }\end{array}$ & $\mathrm{C}_{8} \mathrm{H}_{10}$ & $886.14,2.371$ & 106.0777 & 41103 & \\
\hline $\begin{array}{l}\text { cis-1-Methyl-2-methylene-3-(1- } \\
\text { methylethyl)cyclopentane }\end{array}$ & $\mathrm{C}_{10} \mathrm{H}_{18}$ & $886.14,3.343$ & 138.1403 & 56863 & \\
\hline $\begin{array}{l}\text { 3-Cyclohexyl-(2S-trans)- } \\
\text { oxiranemethanol }\end{array}$ & $\mathrm{C}_{9} \mathrm{H}_{16} \mathrm{O}_{2} ?$ & $898.143,3.365$ & 156.1145 & 23926 & 3.03E-4 \\
\hline o-Xylene & $\mathrm{C}_{8} \mathrm{H}_{10}$ & $904.145,2.412$ & 106.0777 & 579097 & \\
\hline $\begin{array}{l}\text { cis- } 1,1,2,3- \\
\text { Tetramethylcyclohexane }\end{array}$ & $\mathrm{C}_{10} \mathrm{H}_{20}$ & $940.155,3.462$ & 140.156 & 46357 & \\
\hline Decane & $\mathrm{C}_{10} \mathrm{H}_{22}$ & $976.165,4.063$ & 142.1716 & 3227324 & \\
\hline $\begin{array}{l}\text { 1-Methyl-3-(1-methylethyl)-(1R- } \\
\text { trans)cyclohexane }\end{array}$ & $\mathrm{C}_{10} \mathrm{H}_{20}$ & $988.169,3.576$ & 140.156 & 300712 & \\
\hline 2,4-Dimethyldecane & $\mathrm{C}_{12} \mathrm{H}_{26}$ & $994.171,4.305$ & 170.2029 & 486717 & \\
\hline $\begin{array}{l}\text { trans-1-Ethyl-4- } \\
\text { methylcyclohexane }\end{array}$ & $\mathrm{C}_{9} \mathrm{H}_{18}$ & $1006.17,3.657$ & 126.1403 & 1111823 & \\
\hline (4RS,5S)-5-Methylhept-2-yn-4-ol & $\mathrm{C}_{8} \mathrm{H}_{14} \mathrm{O}$ & $1012.18,3.568$ & 126.1039 & 100396 & \\
\hline 1,5-Dimethylpyrazol-4-amine & $\mathrm{C}_{5} \mathrm{H}_{9} \mathrm{~N}_{3}$ & $1018.18,3.552$ & 111.0791 & 16582 & \\
\hline 1,2-Dimethylbenzene & $\mathrm{C}_{8} \mathrm{H}_{10}$ & $1024.18,2.427$ & 106.0777 & 236358 & \\
\hline 2,6,7-Trimethyldecane & $\mathrm{C}_{13} \mathrm{H}_{28}$ & $1024.18,4.335$ & 184.2186 & 1388995 & \\
\hline $\begin{array}{l}\text { 1-(Hydroxymethyl)-3-methylene- } \\
\text { 1-cyclobutanol }\end{array}$ & $\mathrm{C}_{6} \mathrm{H}_{10} \mathrm{O}_{2}$ & $1036.18,3.603$ & 114.0675 & 21648 & \\
\hline $\begin{array}{l}\text { 1-(3-Cyclopenten-1-yl)-4-methyl- } \\
\text { 4-penten-2-one }\end{array}$ & $\mathrm{C}_{11} \mathrm{H}_{16} \mathrm{O}$ ? & $1054.19,3.303$ & 164.1196 & 23517 & 2.97E-4 \\
\hline 6-Ethyl-2-methyloctane & $\mathrm{C}_{11} \mathrm{H}_{24}$ & $1054.19,4.330$ & 156.1873 & 398225 & \\
\hline 2-Ethyl-1,3-dimethylcyclohexane & $\mathrm{C}_{10} \mathrm{H}_{20}$ & $1072.19,3.636$ & 140.156 & 266101 & \\
\hline 2,7,10-Trimethyldodecane & $\mathrm{C}_{15} \mathrm{H}_{32}$ & $1078.19,4.351$ & 212.2499 & 471610 & \\
\hline Methyl benzenesulfite & $\mathrm{C}_{18} \mathrm{H}_{28} \mathrm{O}_{2} \mathrm{~S}$ & $1084.2,3.863$ & 308.1805 & 19567 & \\
\hline $\begin{array}{l}\text { trans-1-Methyl-2-nonyl- } \\
\text { cyclohexane }\end{array}$ & $\mathrm{C}_{16} \mathrm{H}_{32}$ & $1096.2,3.631$ & 224.2499 & 223780 & \\
\hline $\begin{array}{l}\text { (2S)-1,2-Diphenyl-2- } \\
\text { hydroxyethanone }\end{array}$ & $\mathrm{C}_{14} \mathrm{H}_{12} \mathrm{O}_{2}$ ? & $1102.2,2.577$ & 212.0832 & 12828 & $1.62 \mathrm{E}-4$ \\
\hline 3-Ethyl-2,7-dimethyloctane & $\mathrm{C}_{12} \mathrm{H}_{26}$ & $1102.2,4.325$ & 170.2029 & 329967 & \\
\hline $\begin{array}{l}(1,2- \\
\text { Dimethylcyclopentyl)methanol }\end{array}$ & $\mathrm{C}_{8} \mathrm{H}_{16} \mathrm{O}$ & $1114.2,3.630$ & 128.1196 & 69364 & \\
\hline $\begin{array}{l}\text { 3,3-Dimethylcyclohex-1-en-1-yl } \\
\text { but-2-enoate }\end{array}$ & $\mathrm{C}_{12} \mathrm{H}_{18} \mathrm{O}_{2}$ ? & $1138.21,3.639$ & 194.1301 & 22096 & $2.79 \mathrm{E}-4$ \\
\hline Octadecane & $\mathrm{C}_{18} \mathrm{H}_{38}$ & $1150.22,4.351$ & 254.2968 & 333249 & \\
\hline $\begin{array}{l}\text { 1-(1-Cyclopentenyl)-1-hydroxy- } \\
\text { 5,5-dimethyl-6-hepten-3-one }\end{array}$ & $\mathrm{C}_{14} \mathrm{H}_{22} \mathrm{O}_{2}$ ? & $1168.22,3.947$ & 222.1614 & 80765 & $1.02 \mathrm{E}-3$ \\
\hline 2-Methylnonane & $\mathrm{C}_{10} \mathrm{H}_{22}$ & $1168.22,4.318$ & 142.1716 & 79586 & \\
\hline 4-Methyldecane & $\mathrm{C}_{11} \mathrm{H}_{24}$ & $1192.23,4.377$ & 156.1873 & 498135 & \\
\hline
\end{tabular}




\begin{tabular}{|c|c|c|c|c|c|}
\hline 2-Methyldecane & $\mathrm{C}_{11} \mathrm{H}_{24}$ & $1198.23,4.428$ & 156.1873 & 636624 & \\
\hline Undecane & $\mathrm{C}_{11} \mathrm{H}_{24}$ & $1216.23,4.708$ & 156.1873 & 142311 & \\
\hline $\begin{array}{l}\text { N-Benzyl-N-[(3E)-3,5-hexadien- } \\
\text { 1-yl]amine }\end{array}$ & $\mathrm{C}_{13} \mathrm{H}_{19} \mathrm{~N}$ & $1222.24,2.630$ & 189.1512 & 23869 & \\
\hline 2,6,10,14-Tetramethylhexadecane & $\mathrm{C}_{20} \mathrm{H}_{42}$ & $1228.24,4.352$ & 282.3281 & 103975 & \\
\hline Butylcyclohexane & $\mathrm{C}_{10} \mathrm{H}_{20}$ & $1234.24,3.672$ & 140.156 & 518531 & \\
\hline 3-Methyldecane & $\mathrm{C}_{11} \mathrm{H}_{24}$ & $1240.24,4.366$ & 156.1873 & 614389 & \\
\hline 1-Ethyl-2-methylbenzene & $\mathrm{C}_{9} \mathrm{H}_{12}$ & $1264.25,2.623$ & 120.0934 & 245105 & \\
\hline (1-Methylpropyl)cyclooctane & $\mathrm{C}_{12} \mathrm{H}_{24}$ & $1276.25,3.965$ & 168.1873 & 266885 & \\
\hline Pentadecane & $\mathrm{C}_{15} \mathrm{H}_{32}$ & $1282.25,4.641$ & 212.2499 & 75451 & \\
\hline 1,2,3-Trimethylbenzene & $\mathrm{C}_{9} \mathrm{H}_{12}$ & $1288.25,2.643$ & 120.0934 & 704973 & \\
\hline Crotonaldehyde & $\mathrm{C}_{4} \mathrm{H}_{6} \mathrm{O}$ & $1288.25,3.919$ & 70.0413 & 3562 & \\
\hline trans-1,2-Dibutylcyclopropane & $\mathrm{C}_{11} \mathrm{H}_{22}$ & $1300.26,3.912$ & 154.1716 & 24536 & \\
\hline 3-Ethoxy-1-propyne & $\mathrm{C}_{5} \mathrm{H}_{8} \mathrm{O}$ & $1312.26,4.286$ & 84.057 & 22680 & \\
\hline 2-Methylundecane & $\mathrm{C}_{12} \mathrm{H}_{26}$ & $1348.27,4.643$ & 170.2029 & 179003 & \\
\hline Dodecane & $\mathrm{C}_{12} \mathrm{H}_{26}$ & $1366.28,4.402$ & 170.2029 & 4252949 & \\
\hline 4,6-Dimethyldecan-1-ol & $\mathrm{C}_{12} \mathrm{H}_{26} \mathrm{O}$ ? & $1372.28,3.842$ & 186.1978 & 41630 & $5.27 \mathrm{E}-4$ \\
\hline $\begin{array}{l}2,2,4,5 \text {-Tetramethylhex-5-en-3- } \\
\text { one }\end{array}$ & $\mathrm{C}_{10} \mathrm{H}_{14} \mathrm{O}$ ? & $1372.28,4.636$ & 150.1039 & 50266 & $6.36 \mathrm{E}-4$ \\
\hline 1-Isopropyl-4-methylcyclohexane & $\mathrm{C}_{10} \mathrm{H}_{20}$ & $1384.28,3.915$ & 140.156 & 108201 & \\
\hline (2-Methylpropyl)benzene & $\mathrm{C}_{10} \mathrm{H}_{14}$ & $1402.29,2.823$ & 134.109 & 15150 & \\
\hline 2,4,6,8-Tetramethylundecene & $\mathrm{C}_{15} \mathrm{H}_{30}$ & $1414.29,4.211$ & 210.2342 & 53998 & \\
\hline 1-Ethyl-2-propylcyclohexane & $\mathrm{C}_{11} \mathrm{H}_{22}$ & $1438.3,3.924$ & 154.1716 & 309499 & \\
\hline 3,7-Dimethyldecane & $\mathrm{C}_{12} \mathrm{H}_{26}$ & $1450.3,4.608$ & 170.2029 & 346530 & \\
\hline (2S,6R)-2,6-Dimethyl-1-octanol & $\mathrm{C}_{10} \mathrm{H}_{22} \mathrm{O}$ ? & $1456.3,4.256$ & 158.1665 & 25946 & $3.28 \mathrm{E}-4$ \\
\hline Decahydrono hyphennaphthalene & $\mathrm{C}_{10} \mathrm{H}_{18}$ & $1468.31,3.336$ & 138.1403 & 172419 & \\
\hline Heptadecane & $\mathrm{C}_{17} \mathrm{H}_{36}$ & $1468.31,4.639$ & 240.2812 & 218241 & \\
\hline $\begin{array}{l}\text { 1-Methyl-2-(1- } \\
\text { methylethyl)benzene }\end{array}$ & $\mathrm{C}_{10} \mathrm{H}_{14}$ & $1492.31,2.756$ & 134.109 & 78170 & \\
\hline $\begin{array}{l}\text { 1-Hydroxymethyl-1,3-dimethyl- } \\
\text { cyclopentane }\end{array}$ & $\mathrm{C}_{8} \mathrm{H}_{16} \mathrm{O}$ & $1516.32,3.894$ & 128.1196 & 46608 & \\
\hline $\begin{array}{l}\text { 6-Ethyl-2-methylno } \\
\text { hyphendecane }\end{array}$ & $\mathrm{C}_{13} \mathrm{H}_{28}$ & $1528.32,4.587$ & 184.2186 & 218682 & \\
\hline $\begin{array}{l}\text { (1R,3S)-7,7-Dimethyl-2- } \\
\text { oxabicyclo[4.1.1.]octan-3-one }\end{array}$ & $\mathrm{C}_{9} \mathrm{H}_{14} \mathrm{O}_{2}$ & $1540.33,4.097$ & 154.0988 & 12981 & \\
\hline
\end{tabular}




\begin{tabular}{|c|c|c|c|c|c|}
\hline Docosane & $\mathrm{C}_{22} \mathrm{H}_{46}$ & $1546.33,4.604$ & 310.3594 & 133127 & \\
\hline 2-But-1-enyltetrahydrofuran & $\mathrm{C}_{8} \mathrm{H}_{14} \mathrm{O}$ & $1552.33,4.149$ & 126.1039 & 69215 & \\
\hline 2,6-Dimethylundecane & $\mathrm{C}_{13} \mathrm{H}_{28}$ & $1570.34,4.582$ & 184.2186 & 1126021 & \\
\hline 2-Propyldecan-1-ol & $\mathrm{C}_{13} \mathrm{H}_{28} \mathrm{O}$ ? & $1582.34,4.834$ & 200.2135 & 13463 & $1.70 \mathrm{E}-4$ \\
\hline 1-Ethyl-4-methylbenzene & $\mathrm{C}_{9} \mathrm{H}_{12}$ & $1588.34,2.602$ & 120.0934 & 104961 & \\
\hline 4-Methylundecane & $\mathrm{C}_{12} \mathrm{H}_{26}$ & $1594.34,4.577$ & 170.2029 & 416818 & \\
\hline Octyl pentyl ether & $\mathrm{C}_{13} \mathrm{H}_{28} \mathrm{O}$ ? & $1606.35,4.870$ & 200.2135 & 46864 & $5.93 \mathrm{E}-4$ \\
\hline Tetradecane & $\mathrm{C}_{14} \mathrm{H}_{30}$ & $1612.35,4.879$ & 198.2342 & 147902 & \\
\hline 1-Methyl-3-propylbenzene & $\mathrm{C}_{10} \mathrm{H}_{14}$ & $1624.35,2.787$ & 134.109 & 148209 & \\
\hline 2,3-Dimethylundecane & $\mathrm{C}_{13} \mathrm{H}_{28}$ & $1630.35,4.524$ & 184.2186 & 86873 & \\
\hline Methylbenzeneacetaldehyde & $\mathrm{C}_{9} \mathrm{H}_{10} \mathrm{O}$ & $1636.35,2.795$ & 134.0726 & 58973 & \\
\hline 3-Methylundecane & $\mathrm{C}_{12} \mathrm{H}_{26}$ & $1642.36,4.577$ & 170.2029 & 642600 & \\
\hline Dibutyldisulfide & $\mathrm{C}_{8} \mathrm{H}_{18} \mathrm{~S}_{2}$ & $1648.36,4.810$ & 178.0844 & 36075 & \\
\hline Pentylcyclohexane & $\mathrm{C}_{11} \mathrm{H}_{22}$ & $1654.36,3.867$ & 154.1716 & 482326 & \\
\hline $\begin{array}{l}\text { 4-Hydroxy-3-methyleneoctan-2- } \\
\text { one }\end{array}$ & $\mathrm{C}_{9} \mathrm{H}_{16} \mathrm{O}_{2}$ ? & $1654.36,4.116$ & 156.1145 & 17649 & $2.23 \mathrm{E}-4$ \\
\hline Octacosane & $\mathrm{C}_{28} \mathrm{H}_{58}$ & $1654.36,4.813$ & 394.4533 & 69966 & \\
\hline Decahydro-2-methylnaphthalene & $\mathrm{C}_{11} \mathrm{H}_{20}$ & $1660.36,3.545$ & 152.156 & 187546 & \\
\hline 1-Isopropyl-1-methylcyclohexane & $\mathrm{C}_{10} \mathrm{H}_{20}$ & $1660.36,4.167$ & 140.156 & 59014 & \\
\hline 4-Ethyl-1,2-dimethylbenzene & $\mathrm{C}_{10} \mathrm{H}_{14}$ & $1672.37,2.746$ & 134.109 & 304660 & \\
\hline Tricosane & $\mathrm{C}_{23} \mathrm{H}_{48}$ & $1672.37,4.820$ & 324.3751 & 41226 & \\
\hline Hexyl butyl ether & $\mathrm{C}_{10} \mathrm{H}_{22} \mathrm{O}$ ? & $1678.37,4.765$ & 158.1665 & 49482 & $6.26 \mathrm{E}-4$ \\
\hline 5-Methyl-2(3H)-benzoxazolone & $\mathrm{C}_{8} \mathrm{H}_{7} \mathrm{NO}_{2}$ & $1690.37,3.525$ & 149.0471 & 18450 & \\
\hline 1,17-Octadecadiene & $\mathrm{C}_{18} \mathrm{H}_{34}$ & $1720.38,4.056$ & 250.2655 & 31847 & \\
\hline 2,4-Dimethyl-1,3-hexanediol & $\mathrm{C}_{8} \mathrm{H}_{18} \mathrm{O}_{2}$ & $1720.38,4.412$ & 146.1301 & 32388 & \\
\hline Hexacosane & $\mathrm{C}_{26} \mathrm{H}_{54}$ & $1738.38,4.778$ & 366.422 & 314161 & \\
\hline 1-Methyldecahydronaphthalene & $\mathrm{C}_{11} \mathrm{H}_{20}$ & $1756.39,3.482$ & 152.156 & 91029 & \\
\hline 3-Acetyl-3-methylcyclohexanone & $\mathrm{C}_{9} \mathrm{H}_{14} \mathrm{O}_{2}$ ? & $1768.39,4.388$ & 154.0988 & 27720 & $3.51 \mathrm{E}-4$ \\
\hline Tridecane & $\mathrm{C}_{13} \mathrm{H}_{28}$ & $1774.39,4.526$ & 184.2186 & 5273133 & \\
\hline $\begin{array}{l}\text { 1-Methyl-3-(1- } \\
\text { methylethyl)benzene }\end{array}$ & $\mathrm{C}_{10} \mathrm{H}_{14}$ & $1780.4,2.746$ & 134.109 & 153443 & \\
\hline
\end{tabular}




\begin{tabular}{|c|c|c|c|c|c|}
\hline $\begin{array}{l}\text { trans-1-Methyl-2- } \\
\text { octylcyclopropane }\end{array}$ & $\mathrm{C}_{12} \mathrm{H}_{24}$ & $1786.4,3.998$ & 168.1873 & 23564 & \\
\hline 4-Methylphenyl acetone & $\mathrm{C}_{10} \mathrm{H}_{12} \mathrm{O} ?$ & $1792.4,2.940$ & 148.0883 & 79794 & $1.01 \mathrm{E}-4$ \\
\hline 1-Methyl-3-pentylcyclohexane & $\mathrm{C}_{12} \mathrm{H}_{24}$ & $1822.41,4.089$ & 168.1873 & 448065 & \\
\hline $\begin{array}{l}\text { 1-Methyl-4-(1- } \\
\text { methylpropyl)benzene }\end{array}$ & $\mathrm{C}_{11} \mathrm{H}_{16}$ & $1828.41,2.880$ & 148.1247 & 51320 & \\
\hline $\begin{array}{l}\text { 5-Hydroxy-1,2,3,4- } \\
\text { tetrahydronaphthalen-1-one }\end{array}$ & $\mathrm{C}_{10} \mathrm{H}_{12} \mathrm{O}_{2} ?$ & $1840.41,3.767$ & 164.0832 & 17659 & $2.23 \mathrm{E}-4$ \\
\hline $\begin{array}{l}\text { 3-(Hex-1'-enyl)cyclopropane-2,2- } \\
\text { dimethylcyclopropane-1- } \\
\text { carbaldehyde }\end{array}$ & $\mathrm{C}_{12} \mathrm{H}_{20} \mathrm{O}$ ? & $1858.42,3.679$ & 180.1509 & 63752 & $8.07 \mathrm{E}-4$ \\
\hline 1,4-Dicyclohexylbutane & $\mathrm{C}_{16} \mathrm{H}_{30}$ & $1858.42,4.032$ & 222.2342 & 157119 & \\
\hline 3-Methyldodecane & $\mathrm{C}_{13} \mathrm{H}_{28}$ & $1882.43,4.715$ & 184.2186 & 61465 & \\
\hline $\begin{array}{l}\text { 2,4-Dimethyl-1-(1- } \\
\text { methylethyl)benzene }\end{array}$ & $\mathrm{C}_{11} \mathrm{H}_{16}$ & $1888.43,2.846$ & 148.1247 & 45377 & \\
\hline $\begin{array}{l}\text { Methyl p-(isopropyl)benzyl } \\
\text { sulfoxide }\end{array}$ & $\mathrm{C}_{11} \mathrm{H}_{16} \mathrm{OS}$ ? & $1894.43,2.836$ & 196.0916 & 12872 & $1.63 \mathrm{E}-4$ \\
\hline 2,2-Dimethylpropyl nitrite & $\mathrm{C}_{5} \mathrm{H}_{11} \mathrm{NO}_{2}$ & $1930.44,4.938$ & 117.0784 & 28775 & \\
\hline (2-Methylpropyl)cyclohexane & $\mathrm{C}_{10} \mathrm{H}_{20}$ & $1942.44,3.967$ & 140.156 & 98655 & \\
\hline Hentriacontane & $\mathrm{C}_{31} \mathrm{H}_{64}$ & $1954.45,4.639$ & 436.5003 & 109177 & \\
\hline 1,2,3,4-Tetramethylbenzene & $\mathrm{C}_{10} \mathrm{H}_{14}$ & $1960.45,2.746$ & 134.109 & 103921 & \\
\hline 1-Methylcyclohexyl acetate & $\mathrm{C}_{9} \mathrm{H}_{16} \mathrm{O}_{2} ?$ & $1960.45,4.325$ & 156.1145 & 19955 & $2.52 \mathrm{E}-4$ \\
\hline 6-Methyldodecane & $\mathrm{C}_{13} \mathrm{H}_{28}$ & $1972.45,4.629$ & 184.2186 & 791480 & \\
\hline 2,4-Dimethylundecane & $\mathrm{C}_{13} \mathrm{H}_{28}$ & $1984.45,4.613$ & 184.2186 & 228051 & \\
\hline Dotriacontane & $\mathrm{C}_{32} \mathrm{H}_{66}$ & $1990.46,4.871$ & 450.5159 & 19610 & \\
\hline $\begin{array}{l}\text { 6-Hexyl-4-oxa-5- } \\
\text { azaspiro[2.4]hept-5-ene }\end{array}$ & $\mathrm{C}_{11} \mathrm{H}_{19} \mathrm{NO}$ & $2002.46,3.883$ & 181.1461 & 13384 & \\
\hline 4-Methyldodecane & $\mathrm{C}_{13} \mathrm{H}_{28}$ & $2002.46,4.618$ & 184.2186 & 339381 & \\
\hline 2,3,7-Trimethyloctane & $\mathrm{C}_{11} \mathrm{H}_{24}$ & $2008.46,4.906$ & 156.1873 & 1178836 & \\
\hline 2-Methyldodecane & $\mathrm{C}_{13} \mathrm{H}_{28}$ & $2014.46,4.644$ & 184.2186 & 685733 & \\
\hline $\begin{array}{l}\text { 1-(1,2-Dimethylcyclopentyl)-, } \\
\text { trans-2-propyn-1-one }\end{array}$ & $\mathrm{C}_{10} \mathrm{H}_{14} \mathrm{O} ?$ & $2032.47,3.866$ & 150.1039 & 27671 & $3.50 \mathrm{E}-4$ \\
\hline 1-(2-Ethylphenyl)ethan-1-one & $\mathrm{C}_{10} \mathrm{H}_{12} \mathrm{O}$ ? & $2038.47,2.809$ & 148.0883 & 33101 & $4.19 \mathrm{E}-4$ \\
\hline (1-Methylbutyl)benzene & $\mathrm{C}_{11} \mathrm{H}_{16}$ & $2068.48,2.875$ & 148.1247 & 24459 & \\
\hline Hexylcyclohexane & $\mathrm{C}_{12} \mathrm{H}_{24}$ & $2086.48,3.914$ & 168.1873 & 533328 & \\
\hline 1-(4-Methylphenyl)-1-propanone & $\mathrm{C}_{10} \mathrm{H}_{12} \mathrm{O}$ ? & $2098.49,2.851$ & 148.0883 & 44746 & $5.66 \mathrm{E}-4$ \\
\hline $\begin{array}{l}\text { cis,trans-2- } \\
\text { Ethylbicyclo[4.4.0]decane }\end{array}$ & $\mathrm{C}_{12} \mathrm{H}_{22}$ & $2158.5,3.549$ & 166.1716 & 90645 & \\
\hline (1,1-Dimethylbutyl)benzene & $\mathrm{C}_{12} \mathrm{H}_{18}$ & $2170.51,2.974$ & 162.1403 & 62336 & \\
\hline
\end{tabular}




\begin{tabular}{|c|c|c|c|c|c|}
\hline Methyl(1-methylethyl)benzene & $\mathrm{C}_{10} \mathrm{H}_{14}$ & $2176.51,2.649$ & 134.109 & 25755 & \\
\hline $\begin{array}{l}\text { 2-(4'-Methylphenyl)no } \\
\text { hyphenpropanal }\end{array}$ & $\mathrm{C}_{10} \mathrm{H}_{12} \mathrm{O}$ ? & $2182.51,2.803$ & 148.0883 & 85326 & $1.08 \mathrm{E}-3$ \\
\hline 6-Methylundecane & $\mathrm{C}_{12} \mathrm{H}_{26}$ & $2194.51,4.733$ & 170.2029 & 130169 & \\
\hline $\begin{array}{l}\text { 3,4- } \\
\text { Trimethylbenzeneacetaldehyde }\end{array}$ & $\mathrm{C}_{11} \mathrm{H}_{14} \mathrm{O}$ ? & $2200.52,2.916$ & 162.1039 & 35227 & 4.46E-4 \\
\hline $\begin{array}{l}\text { 4-Methyl-1-[4-(1-methylethenyl)- } \\
\text { 1-cyclohexen-1-yl]-3-penten-1- } \\
\text { one }\end{array}$ & $\mathrm{C}_{15} \mathrm{H}_{22} \mathrm{O}$ ? & $2200.52,3.253$ & 218.1665 & 11023 & $1.39 \mathrm{E}-4$ \\
\hline $\begin{array}{l}\text { 2-Methyl-2-(1-methyl-1- } \\
\text { propenyl)no } \\
\text { hyphencyclobutanone }\end{array}$ & $\mathrm{C}_{9} \mathrm{H}_{14} \mathrm{O} ?$ & $2200.52,3.486$ & 138.1039 & 10705 & $1.35 \mathrm{E}-4$ \\
\hline 2-Methyl-4-hexyn-3-ol & $\mathrm{C}_{7} \mathrm{H}_{12} \mathrm{O} ?$ & $2218.52,4.334$ & 112.0883 & 16223 & $2.05 \mathrm{E}-4$ \\
\hline 1-Methyl-3-propylcyclooctane & $\mathrm{C}_{12} \mathrm{H}_{24}$ & $2224.52,4.143$ & 168.1873 & 155854 & \\
\hline $\begin{array}{l}\text { Decahydro-1,1,6- } \\
\text { trimethylnaphthalene }\end{array}$ & $\mathrm{C}_{13} \mathrm{H}_{24}$ & $2266.54,3.695$ & 180.1873 & 17481 & \\
\hline 2,2-Dimethyl-5-hexen-3-one & $\mathrm{C}_{8} \mathrm{H}_{14} \mathrm{O}$ & $2296.54,4.856$ & 126.1039 & 30082 & \\
\hline Hexadecane & $\mathrm{C}_{16} \mathrm{H}_{34}$ & $2302.55,4.631$ & 226.2655 & 148714 & \\
\hline 1-Ehyl-4-(1-methylethyl)benzene & $\mathrm{C}_{11} \mathrm{H}_{16}$ & $2308.55,2.762$ & 148.1247 & 36205 & \\
\hline (1,2-Dimethylpropyl)cyclohexane & $\mathrm{C}_{11} \mathrm{H}_{22}$ & $2320.55,4.052$ & 154.1716 & 75779 & \\
\hline (E)-Deca-1,6-dien-3-one & $\mathrm{C}_{10} \mathrm{H}_{16} \mathrm{O}$ ? & $2326.55,4.865$ & 152.1196 & 9930 & $1.25 \mathrm{E}-4$ \\
\hline 1,2,3,4-Tetrahydronaphthalene & $\mathrm{C}_{10} \mathrm{H}_{12}$ & $2332.55,2.464$ & 132.0934 & 23183 & \\
\hline 1-Ethyl-2-isopropenyl-benzene & $\mathrm{C}_{11} \mathrm{H}_{14}$ & $2332.55,2.664$ & 146.109 & 21149 & \\
\hline $\begin{array}{l}\text { cis-1-Methyl-4-(1-methylethyl) } \\
\text { cyclohexane }\end{array}$ & $\mathrm{C}_{10} \mathrm{H}_{20}$ & $2332.55,4.006$ & 140.156 & 101476 & \\
\hline $\begin{array}{l}\text { 6,8-Dimethylbicyclo[3.2.2]nona- } \\
\text { 6,8-dien-3-one }\end{array}$ & $\mathrm{C}_{11} \mathrm{H}_{14} \mathrm{O}$ ? & $2344.56,2.917$ & 162.1039 & 40791 & $5.16 \mathrm{E}-4$ \\
\hline 1,10-Dicyanonohyphen-decane & $\mathrm{C}_{12} \mathrm{H}_{20} \mathrm{~N}_{2}$ & $2362.56,3.980$ & 192.1621 & 47913 & \\
\hline $\begin{array}{l}\text { (cis)-1-Butyl-2- } \\
\text { undecylcyclopropane }\end{array}$ & $\mathrm{C}_{18} \mathrm{H}_{36}$ & $2368.56,4.225$ & 252.2812 & 47597 & \\
\hline 6-Methyltridecane & $\mathrm{C}_{14} \mathrm{H}_{30}$ & $2368.56,4.587$ & 198.2342 & 748800 & \\
\hline $\begin{array}{l}\text { (2S)-4-(Isopropylamino)-2- } \\
\text { butanol }\end{array}$ & $\mathrm{C}_{7} \mathrm{H}_{17} \mathrm{NO}$ & $2374.57,2.645$ & 131.1305 & 8496 & \\
\hline $\begin{array}{l}\text { (E)-4-Methyl-6-(2',5',6',6'- } \\
\text { tetramethylcyclohex-1'-enyl)hex- } \\
\text { 3-en-1-ol }\end{array}$ & $\mathrm{C}_{17} \mathrm{H}_{30} \mathrm{O}$ ? & $2374.57,3.669$ & 250.2291 & 28495 & $3.60 \mathrm{E}-4$ \\
\hline N-(But-2-enoyl)prop-2-enamide & $\mathrm{C}_{7} \mathrm{H}_{11} \mathrm{NO}$ & $2374.57,4.136$ & 125.0835 & 16764 & \\
\hline 2,6,11-Trimethyldodecane & $\mathrm{C}_{15} \mathrm{H}_{32}$ & $2404.58,4.856$ & 212.2499 & 223726 & \\
\hline $\begin{array}{l}\text { (Z)-5-Methylundec-4-en-6-yn-3- } \\
\text { ol }\end{array}$ & $\mathrm{C}_{12} \mathrm{H}_{20} \mathrm{O}$ ? & $2428.58,3.667$ & 180.1509 & 5147 & $6.52 \mathrm{E}-5$ \\
\hline 3-Methyltridecane & $\mathrm{C}_{14} \mathrm{H}_{30}$ & $2458.59,4.515$ & 198.2342 & 628531 & \\
\hline $\begin{array}{l}\mathrm{N}-(1 \text {-Adamantyl)oxy-N,N- } \\
\text { dibutylamine }\end{array}$ & $\mathrm{C}_{18} \mathrm{H}_{33} \mathrm{NO}$ & $2464.59,3.185$ & 279.2557 & 15333 & \\
\hline
\end{tabular}




\begin{tabular}{|c|c|c|c|c|c|}
\hline $\begin{array}{l}\text { 1-Ethyl-4-(1- } \\
\text { methylpropyl)benzene }\end{array}$ & $\mathrm{C}_{12} \mathrm{H}_{18}$ & $2500.6,2.893$ & 162.1403 & 21455 & \\
\hline $\begin{array}{l}\text { trans-1-[(2- } \\
\text { Butylcyclopropyl)methyl]benzene }\end{array}$ & $\mathrm{C}_{14} \mathrm{H}_{20}$ & $2506.6,2.570$ & 188.156 & 10559 & \\
\hline Heptylcyclohexane & $\mathrm{C}_{13} \mathrm{H}_{26}$ & $2506.6,3.903$ & 182.2029 & 601725 & \\
\hline $\begin{array}{l}\text { trans-1-Ethyl-1,4-dimethyl } \\
\text { cyclohexane }\end{array}$ & $\mathrm{C}_{10} \mathrm{H}_{20}$ & $2524.61,4.167$ & 140.156 & 85062 & \\
\hline $\begin{array}{l}\text { 2,2-Dimethyl-4-(methylethyl)- } \\
\text { 2H-imidazole }\end{array}$ & $\mathrm{C}_{8} \mathrm{H}_{14} \mathrm{~N}_{2}$ & $2536.61,4.096$ & 138.1151 & 40855 & \\
\hline 2-(1-Phenylethyl)phenol & $\mathrm{C}_{14} \mathrm{H}_{14} \mathrm{O}$ ? & $2536.61,4.676$ & 198.1039 & 94951 & $1.20 \mathrm{E}-3$ \\
\hline 3-Methyl-1-hexen-5-one & $\mathrm{C}_{7} \mathrm{H}_{12} \mathrm{O}$ & $2620.64,4.311$ & 112.0883 & 29103 & \\
\hline 2-Pent-1-enyltetrahydrofuran & $\mathrm{C}_{9} \mathrm{H}_{16} \mathrm{O} ?$ & $2722.67,3.981$ & 140.1196 & 25651 & $3.24 \mathrm{E}-4$ \\
\hline 1-Azido-1-methylcyclohexane & $\mathrm{C}_{7} \mathrm{H}_{13} \mathrm{~N}_{3}$ & $2734.67,3.982$ & 139.1104 & 92533 & \\
\hline $\begin{array}{l}\text { 1,2,3,4-Tetrahydro-5-methyl- } \\
\text { naphthalene }\end{array}$ & $\mathrm{C}_{11} \mathrm{H}_{14}$ & $2746.67,2.525$ & 146.109 & 62660 & \\
\hline (7Z,11Z)-Hexadecadienal & $\mathrm{C}_{16} \mathrm{H}_{28} \mathrm{O}$ ? & $2746.67,4.272$ & 236.2135 & 37286 & 4.72E-4 \\
\hline 5-Propyldecane & $\mathrm{C}_{13} \mathrm{H}_{28}$ & $2746.67,4.546$ & 184.2186 & 693002 & \\
\hline $\begin{array}{l}\text { 2,6,10,14- } \\
\text { Tetramethylheptadecane }\end{array}$ & $\mathrm{C}_{21} \mathrm{H}_{44}$ & $2752.68,4.762$ & 296.3438 & 1130039 & \\
\hline $\begin{array}{l}\text { 1,2,3,4-Tetrahydro-1,8- } \\
\text { dimethylnaphthalene }\end{array}$ & $\mathrm{C}_{12} \mathrm{H}_{16}$ & $2764.68,2.686$ & 160.1247 & 37045 & \\
\hline 5-Methyltetradecane & $\mathrm{C}_{15} \mathrm{H}_{32}$ & $2764.68,4.510$ & 212.2499 & 312547 & \\
\hline $\begin{array}{l}\text { (2S,3S)-2-Methyl-3-(2-methyl-2- } \\
\text { propenyl)cyclopentanone }\end{array}$ & $\mathrm{C}_{10} \mathrm{H}_{16} \mathrm{O} ?$ & $2776.68,4.136$ & 152.1196 & 28774 & $3.64 \mathrm{E}-4$ \\
\hline 4-Methyltetradecane, & $\mathrm{C}_{15} \mathrm{H}_{32}$ & $2782.68,4.525$ & 212.2499 & 286396 & \\
\hline $\begin{array}{l}\text { 3-Methylene-5-(2-furyl)penten-5- } \\
\text { ol }\end{array}$ & $\mathrm{C}_{10} \mathrm{H}_{12} \mathrm{O}_{2}$ ? & $2806.69,4.132$ & 164.0832 & 19152 & $2.42 \mathrm{E}-4$ \\
\hline Tritriacontane & $\mathrm{C}_{33} \mathrm{H}_{68}$ & $2824.7,4.436$ & 464.5316 & 57662 & \\
\hline $\begin{array}{l}\text { 4,4,7,7-Tetramethyl-1,9- } \\
\text { decadiene }\end{array}$ & $\mathrm{C}_{14} \mathrm{H}_{26}$ & $2830.7,4.108$ & 194.2029 & 33496 & \\
\hline 2,6-Dimethylheptadecane & $\mathrm{C}_{19} \mathrm{H}_{40}$ & $2836.7,4.474$ & 268.3125 & 511742 & \\
\hline Isopropoxy-3-methylbenzyl & $\mathrm{C}_{11} \mathrm{H}_{16} \mathrm{O}$ ? & $2848.7,2.959$ & 164.1196 & 9901 & $1.25 \mathrm{E}-4$ \\
\hline Bicyclo[4.3.1]deca-1-ol & $\mathrm{C}_{10} \mathrm{H}_{18} \mathrm{O}$ ? & $2896.72,4.124$ & 154.1352 & 4684 & $5.93 \mathrm{E}-5$ \\
\hline 2,2-Dimethylbutane & $\mathrm{C}_{6} \mathrm{H}_{14}$ & $2902.72,4.881$ & 86.109 & 29381 & \\
\hline $\begin{array}{l}\text { 1,2,3,4-Tetrahydro-6- } \\
\text { methylnaphthalene }\end{array}$ & $\mathrm{C}_{11} \mathrm{H}_{14}$ & $2908.72,2.456$ & 146.109 & 21882 & \\
\hline Octylcyclohexane & $\mathrm{C}_{14} \mathrm{H}_{28}$ & $2908.72,3.878$ & 196.2186 & 538856 & \\
\hline (1E)-1-Hexenylbenzene & $\mathrm{C}_{12} \mathrm{H}_{16}$ & $2914.72,2.633$ & 160.1247 & 9885 & \\
\hline 2,7-Dimethylindan-1-one & $\mathrm{C}_{11} \mathrm{H}_{12} \mathrm{O} ?$ & $2962.74,2.582$ & 160.0883 & 14319 & $1.81 \mathrm{E}-4$ \\
\hline Methyl pentanoate & $\mathrm{C}_{6} \mathrm{H}_{12} \mathrm{O}_{2}$ & $2968.74,4.604$ & 116.0832 & 67230 & \\
\hline
\end{tabular}




\begin{tabular}{|c|c|c|c|c|c|}
\hline 5-Methyl-3-hexyn-2-ol & $\mathrm{C}_{7} \mathrm{H}_{12} \mathrm{O}$ & $2974.74,4.033$ & 112.0883 & 13239 & \\
\hline Cyclopentanodec-5-ene & $\mathrm{C}_{15} \mathrm{H}_{28}$ & $2986.74,4.256$ & 208.2186 & 17694 & \\
\hline 1-Methylnaphthalene & $\mathrm{C}_{11} \mathrm{H}_{10}$ & $3022.75,2.319$ & 142.0777 & 62507 & \\
\hline $\begin{array}{l}\text { 4-tert-Butyl-2- } \\
\text { methylenebutyrolactone }\end{array}$ & $\mathrm{C}_{9} \mathrm{H}_{14} \mathrm{O}_{2} ?$ & $3022.75,4.227$ & 154.0988 & 12283 & $1.55 \mathrm{E}-4$ \\
\hline 1-Methyl-2-pentylcyclohexane & $\mathrm{C}_{12} \mathrm{H}_{24}$ & $3106.78,3.970$ & 168.1873 & 122199 & \\
\hline 6-Methylpentadecane & $\mathrm{C}_{16} \mathrm{H}_{34}$ & $3112.78,4.469$ & 226.2655 & 887321 & \\
\hline $\begin{array}{l}\text { 1-N-Butyl-1,2,3,4- } \\
\text { tetrahydronaphthalene }\end{array}$ & $\mathrm{C}_{14} \mathrm{H}_{20}$ & $3118.78,2.526$ & 188.156 & 9956 & \\
\hline 5-Phenylpent-1-en-3-yne & $\mathrm{C}_{11} \mathrm{H}_{10}$ & $3124.78,2.280$ & 142.0777 & 26994 & \\
\hline 2,4,4-Trimethylhexane & $\mathrm{C}_{9} \mathrm{H}_{20}$ & $3124.78,4.668$ & 128.156 & 59807 & \\
\hline 5-Methylpentadecane & $\mathrm{C}_{16} \mathrm{H}_{34}$ & $3130.78,4.438$ & 226.2655 & 285991 & \\
\hline Dioctyldisulfide & $\mathrm{C}_{16} \mathrm{H}_{34} \mathrm{~S}_{2}$ & $3136.79,4.623$ & 290.2096 & 29323 & \\
\hline Eicosane & $\mathrm{C}_{20} \mathrm{H}_{42}$ & $3142.79,4.644$ & 282.3281 & 167389 & \\
\hline 4-Methylpentadecane & $\mathrm{C}_{16} \mathrm{H}_{34}$ & $3148.79,4.449$ & 226.2655 & 312879 & \\
\hline 2-Methylnonadecane & $\mathrm{C}_{20} \mathrm{H}_{42}$ & $3166.79,4.449$ & 282.3281 & 512566 & \\
\hline $\begin{array}{l}\text { (5S,7S,9S,E)-S-Ethyl 5,7,9- } \\
\text { trimethyltridec-3-enethioate }\end{array}$ & $\mathrm{C}_{18} \mathrm{H}_{34} \mathrm{OS}$ & $3196.8,4.063$ & 298.2325 & 14236 & \\
\hline 2,6-Dimethyloctadecane & $\mathrm{C}_{20} \mathrm{H}_{42}$ & $3202.8,4.397$ & 282.3281 & 394596 & \\
\hline $\begin{array}{l}\text { 2-Methyl-5-(2-methyl-3- } \\
\text { methylenebicyclo[2.2.2]oct-5-en- } \\
\text { 2-yl)-2-penten-1-ol }\end{array}$ & $\mathrm{C}_{16} \mathrm{H}_{24} \mathrm{O}$ ? & $3226.81,2.961$ & 232.1822 & 9377 & $1.18 \mathrm{E}-4$ \\
\hline $\begin{array}{l}\text { (1S,3R,4R)-3-(Hydroxymethyl)- } \\
\text { 2-methyl-2- } \\
\text { azabicyclo[2.2.1] heptane }\end{array}$ & $\mathrm{C}_{8} \mathrm{H}_{15} \mathrm{NO}$ & $3238.81,4.064$ & 141.1148 & 15482 & \\
\hline Germacrane-C & $\mathrm{C}_{15} \mathrm{H}_{30}$ & $3244.82,4.077$ & 210.2342 & 52474 & \\
\hline $\begin{array}{l}\text { 1,2,3,4-Tetrahydro-5,6- } \\
\text { dimethylnaphthalene }\end{array}$ & $\mathrm{C}_{12} \mathrm{H}_{16}$ & $3274.82,2.504$ & 160.1247 & 59251 & \\
\hline 1,1-Bis(2,3-epoxypropyl)ethane & $\mathrm{C}_{8} \mathrm{H}_{14} \mathrm{O}_{2}$ & $3274.82,3.892$ & 142.0988 & 16459 & \\
\hline Cyclohexyltoluene & $\mathrm{C}_{13} \mathrm{H}_{18}$ & $3316.84,2.658$ & 174.1403 & 10213 & \\
\hline $\begin{array}{l}2,6,10,15- \\
\text { Tetramethylheptadecane }\end{array}$ & $\mathrm{C}_{21} \mathrm{H}_{44}$ & $3334.84,4.683$ & 296.3438 & 73958 & \\
\hline $\begin{array}{l}(6 \mathrm{R}, 12 \mathrm{R})-6,12- \\
\text { Dimethylpentadec-1-ene }\end{array}$ & $\mathrm{C}_{17} \mathrm{H}_{34}$ & $3364.85,4.027$ & 238.2655 & 42324 & \\
\hline 1-Hexadecene & $\mathrm{C}_{16} \mathrm{H}_{32}$ & $3370.85,4.010$ & 224.2499 & 58707 & \\
\hline 2-Cyanocyclohexanol & $\mathrm{C}_{7} \mathrm{H}_{11} \mathrm{NO}$ & $3382.86,4.445$ & 125.0835 & 142093 & \\
\hline 1,5-Dimethylnaphthalene & $\mathrm{C}_{12} \mathrm{H}_{12}$ & $3418.87,2.386$ & 156.0934 & 91154 & \\
\hline 5-Methyldodecane & $\mathrm{C}_{13} \mathrm{H}_{28}$ & $3478.88,4.387$ & 184.2186 & 254819 & \\
\hline
\end{tabular}




\begin{tabular}{|c|c|c|c|c|c|}
\hline $\begin{array}{l}\text { 4-(2- } \\
\text { Tetrahydropyranyl)oxybutanal }\end{array}$ & $\mathrm{C}_{9} \mathrm{H}_{16} \mathrm{O}_{3} ?$ & $3496.89,4.776$ & 172.1094 & 32032 & 4.05E-4 \\
\hline 2,6-Dimethylnaphthalene & $\mathrm{C}_{12} \mathrm{H}_{12}$ & $3502.89,2.341$ & 156.0934 & 34882 & \\
\hline $\begin{array}{l}\text { (3R,4S,5R,7R)-3,5,7-Trimethyl- } \\
\text { 1-nonen-4-ol }\end{array}$ & $\mathrm{C}_{12} \mathrm{H}_{24} \mathrm{O}$ ? & $3502.89,4.113$ & 184.1822 & 20580 & $2.60 \mathrm{E}-4$ \\
\hline $\begin{array}{l}\text { 2'-Methyl-spiro[cyclopropane- } \\
\text { 1,1'-ind-2'-ene] }\end{array}$ & $\mathrm{C}_{12} \mathrm{H}_{12}$ & $3520.9,2.326$ & 156.0934 & 76324 & \\
\hline Nonadecane & $\mathrm{C}_{19} \mathrm{H}_{40}$ & $3520.9,4.752$ & 268.3125 & 77574 & \\
\hline $\begin{array}{l}\text { (Z)-3-Ethyl-2,2,5,5-tetramethyl- } \\
\text { 3-hexene }\end{array}$ & $\mathrm{C}_{12} \mathrm{H}_{24}$ & $3550.9,4.065$ & 168.1873 & 25652 & \\
\hline 3-Methylhexadecane & $\mathrm{C}_{17} \mathrm{H}_{36}$ & $3550.9,4.330$ & 240.2812 & 314487 & \\
\hline $\begin{array}{l}\text { 3-Hydroxy-7,7-dimethyl-1,8- } \\
\text { nonadien-5-one }\end{array}$ & $\mathrm{C}_{11} \mathrm{H}_{18} \mathrm{O}_{2}$ ? & $3574.91,4.043$ & 182.1301 & 44986 & $5.69 \mathrm{E}-4$ \\
\hline 1,2-Dimethylnaphthalene & $\mathrm{C}_{12} \mathrm{H}_{12}$ & $3598.92,2.323$ & 156.0934 & 12310 & \\
\hline 2,5-Bis(tetrahydrofuran) & $\mathrm{C}_{8} \mathrm{H}_{14} \mathrm{O}_{2}$ ? & $3616.92,4.668$ & 142.0988 & 56289 & 7.13E-4 \\
\hline 4-Methylheptadecane & $\mathrm{C}_{18} \mathrm{H}_{38}$ & $3826.98,4.351$ & 254.2968 & 199200 & \\
\hline 1,6,7-Trimethylnaphthalene & $\mathrm{C}_{13} \mathrm{H}_{14}$ & $3869,2.380$ & 170.109 & 35149 & \\
\hline $\begin{array}{l}\text { (E)-2-((E)-But-2-enylidene) } \\
\text { hydrazinecarbothioamide }\end{array}$ & $\mathrm{C}_{5} \mathrm{H}_{9} \mathrm{~N}_{3} \mathrm{~S}$ & $3875,2.476$ & 143.0512 & 8889 & \\
\hline 2,9-Dimethyldecane & $\mathrm{C}_{12} \mathrm{H}_{26}$ & $3881,4.296$ & 170.2029 & 281719 & \\
\hline 1,4,6-Trimethylnaphthalene & $\mathrm{C}_{13} \mathrm{H}_{14}$ & $3887,2.385$ & 170.109 & 42239 & \\
\hline 3-Ethyl-3-methylhexane & $\mathrm{C}_{9} \mathrm{H}_{20}$ & $3887,4.436$ & 128.156 & 58412 & \\
\hline Octyl allyl ether & $\mathrm{C}_{11} \mathrm{H}_{22} \mathrm{O} ?$ & $3911.01,4.600$ & 170.1665 & 35634 & 4.51E-4 \\
\hline 1-Decene & $\mathrm{C}_{10} \mathrm{H}_{22}$ & $3917.01,4.424$ & 142.1716 & 9111 & \\
\hline (E)-2-Tetradecen-1-ol & $\mathrm{C}_{14} \mathrm{H}_{28} \mathrm{O} ?$ & $4055.05,3.955$ & 212.2135 & 20536 & $2.60 \mathrm{E}-4$ \\
\hline 10-Methylnonadecane & $\mathrm{C}_{20} \mathrm{H}_{42}$ & $4091.06,4.315$ & 282.3281 & 400638 & \\
\hline 2,6,8-Trimethyldecane & $\mathrm{C}_{13} \mathrm{H}_{28}$ & $4109.06,4.310$ & 184.2186 & 250494 & \\
\hline 5-Methyloctadecane & $\mathrm{C}_{19} \mathrm{H}_{40}$ & $4121.07,4.312$ & 268.3125 & 90957 & \\
\hline 3,8-Dimethylundecane & $\mathrm{C}_{13} \mathrm{H}_{28}$ & $4145.07,4.279$ & 184.2186 & 161605 & \\
\hline Heptacosane & $\mathrm{C}_{27} \mathrm{H}_{56}$ & $4157.08,4.305$ & 380.4377 & 246751 & \\
\hline 4,4'-Dimethyl-1,1'-biphenyl & $\mathrm{C}_{14} \mathrm{H}_{14}$ & $4163.08,2.313$ & 182.109 & 13835 & \\
\hline $\begin{array}{l}\text { 2-(Cyclohexylmethyl)tetrahydro } \\
\text { furan }\end{array}$ & $\mathrm{C}_{11} \mathrm{H}_{20} \mathrm{O} ?$ & $4181.09,4.593$ & 168.1509 & 129608 & $1.64 \mathrm{E}-3$ \\
\hline 4,4-Dimethyloctane & $\mathrm{C}_{10} \mathrm{H}_{22}$ & $4217.1,4.518$ & 142.1716 & 29694 & \\
\hline 9-Hydroxyinalool & $\mathrm{C}_{10} \mathrm{H}_{18} \mathrm{O}_{2}$ ? & $4229.1,4.363$ & 170.1301 & 116819 & $1.47 \mathrm{E}-3$ \\
\hline $\begin{array}{l}\text { 2,3,4,5-Tetrahydro-6- } \\
\text { hexylpyridine }\end{array}$ & $\mathrm{C}_{11} \mathrm{H}_{21} \mathrm{~N}$ & $4271.11,3.740$ & 167.1669 & 21019 & \\
\hline
\end{tabular}




\begin{tabular}{|c|c|c|c|c|}
\hline 2,2,4-Trimethyl-2H-imidazole & $\mathrm{C}_{6} \mathrm{H}_{10} \mathrm{~N}_{2}$ & $4307.12,3.923$ & 110.0838 & 13418 \\
\hline Undecylcyclohexane & $\mathrm{C}_{17} \mathrm{H}_{34}$ & $4319.12,3.754$ & 238.2655 & 227962 \\
\hline Bicyclo[3.2.1] octan-1-ol & $\mathrm{C}_{8} \mathrm{H}_{14} \mathrm{O}$ & $4367.14,3.915$ & 126.1039 & 79966 \\
\hline 2,3,5-Trimethylhexane & $\mathrm{C}_{9} \mathrm{H}_{20}$ & $4421.15,4.270$ & 128.156 & 72903 \\
\hline 2,10-Dimethylundecane & $\mathrm{C}_{13} \mathrm{H}_{28}$ & $4493.17,4.222$ & 184.2186 & 119874 \\
\hline 2-Ethyl-1-hexene & $\mathrm{C}_{8} \mathrm{H}_{18}$ & $4943.3,4.242$ & 114.1403 & 33920 \\
\hline 9-Methyl-1,6-methanofluorene & $\mathrm{C}_{15} \mathrm{H}_{14}$ & $4949.31,2.300$ & 194.109 & 8050 \\
\hline 2,5,9-Trimethyldecane & $\mathrm{C}_{13} \mathrm{H}_{28}$ & $4949.31,4.207$ & 184.2186 & 123503 \\
\hline 2,6,10-Trimethyltridecane & $\mathrm{C}_{16} \mathrm{H}_{34}$ & $5009.32,4.429$ & 226.2655 & 12975 \\
\hline $\begin{array}{l}\text { 1a,9b-Dihydro-1H- } \\
\text { cyclopropa[1]phenanthrene }\end{array}$ & $\mathrm{C}_{15} \mathrm{H}_{12}$ & $5327.41,2.167$ & 192.0934 & 10086 \\
\hline 7-Phenylindene & $\mathrm{C}_{15} \mathrm{H}_{12}$ & $5357.42,2.173$ & 192.0934 & 8498 \\
\hline 2,3,3,4-Tetramethylpentane & $\mathrm{C}_{9} \mathrm{H}_{20}$ & $5501.46,4.162$ & 128.156 & 19106 \\
\hline $\begin{array}{l}\text { Methyl-2-propyl-4-2,5- } \\
\text { dihydrofuran }\end{array}$ & $\mathrm{C}_{8} \mathrm{H}_{14} \mathrm{O}$ & $5975.6,3.779$ & 126.1039 & 37708 \\
\hline $\begin{array}{l}\text { (3R)-3-Phenyl-2,3-dihydro-1H- } \\
\text { isoindol-1-one }\end{array}$ & $\mathrm{C}_{14} \mathrm{H}_{11} \mathrm{NO}$ & $6203.67,1.086$ & 209.0835 & 57662 \\
\hline 2-Isocyano-2-methylpropane & $\mathrm{C}_{5} \mathrm{H}_{9} \mathrm{~N}$ & $6659.8,3.892$ & 83.073 & 13399 \\
\hline $\begin{array}{l}\text { (3S)-3-Phenyl-2,3-dihydro-1H- } \\
\text { isoindol-1-one }\end{array}$ & $\mathrm{C}_{14} \mathrm{H}_{11} \mathrm{NO}$ & $6833.85,1.081$ & 209.0835 & 331509 \\
\hline Benzene & $\mathrm{C}_{6} \mathrm{H}_{6}$ & $7085.92,1.087$ & 78.0464 & 29100 \\
\hline Fumaronitrile & $\mathrm{C}_{4} \mathrm{H}_{2} \mathrm{~N}_{2}$ & $7319.99,1.093$ & 78.0212 & 18870 \\
\hline 1,3-Hexadien-5-yne & $\mathrm{C}_{6} \mathrm{H}_{6}$ & $7349.99,1.092$ & 78.0464 & 18682 \\
\hline $\begin{array}{l}\mathrm{N}-[(1,1- \\
\text { Dimethylethyl)carbonimidoyl]-5- } \\
\text { methyl-4-isoxazolamine }\end{array}$ & $\mathrm{C}_{9} \mathrm{H}_{13} \mathrm{~N}_{3} \mathrm{O}$ & $7938.16,1.209$ & 179.1053 & 5903 \\
\hline
\end{tabular}

